Department of Visual Science,

Institute of Ophthalmology, University College London

\title{
Growth Dynamics in the Developing Lateral Geniculate Nucleus
}

\author{
Adrian Lloyd Williams
}

Submitted in partial fulfilment of the requirements of the degree of Doctor of Philosophy

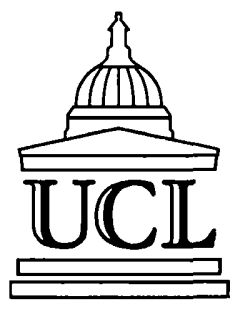


ProQuest Number: 10016061

All rights reserved

\title{
INFORMATION TO ALL USERS
}

The quality of this reproduction is dependent upon the quality of the copy submitted.

In the unlikely event that the author did not send a complete manuscript and there are missing pages, these will be noted. Also, if material had to be removed, a note will indicate the deletion.

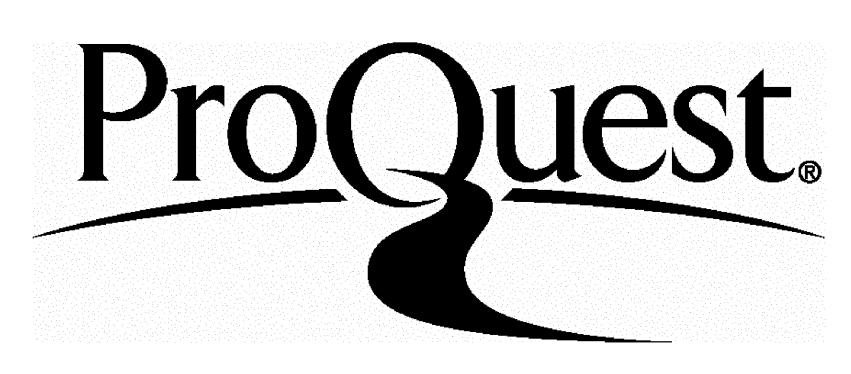

ProQuest 10016061

Published by ProQuest LLC(2016). Copyright of the Dissertation is held by the Author.

All rights reserved.

This work is protected against unauthorized copying under Title 17, United States Code. Microform Edition $\odot$ ProQuest LLC.

\author{
ProQuest LLC \\ 789 East Eisenhower Parkway \\ P.O. Box 1346 \\ Ann Arbor, MI 48106-1346
}




\section{Declaration.}

I certify that this thesis submitted for the degree of Doctor of Philosophy is the result of my own research, except where otherwise acknowledged, and that this thesis (or any part of the same) has not been submitted for a higher degree to any other university or institution.

Signed :

Adrian Lloyd Williams

Date 


\section{Abstract.}

Binocularly segregated maps develop from stages where projections from the two eyes initially overlap. Differential growth of the lateral geniculate nucleus may regulate their segregation. This hypothesis has been tested using novel shape modelling techniques to quantify the 3D structure of the ferret LGN at successive developmental stages. Spatio-temporal aspects of LGN shape change have been compared with different measurements of its cellular population. Mature shape arises prior to the full maturation of its cellular constituents, and is probably influenced by the substantial volumetric expansion which occurs to the nucleus. Cellular bodies contribute little to volumetric expansion, and it is suggested that non-somatic factors such as axon arbors, or the late proliferation of glial cells play a more important role.

The nucleus rotates laterally by $70^{\circ}$ during the first three postnatal weeks. Prior to evaluating spatial shape changes, LGN models were normalised to account for rotation. Specific patterns of shape change transpire, consistent with the nucleus accommodating ocular segregation. Following segregation, only isotropic growth occurs. It has not been possible to show a dependant relationship between LGN growth and ocular segregation, either in terms of model transformations, or local changes between LGN structure and terminal field regions.

The role of retinal afferents in regulating LGN shape and its cellular population have been explored using monocular enucleation. Varying degrees of abnormalities occur to the cellular population, but contributions made by cells to LGN growth are similar to the normal animal. LGN structure does not develop fully, and the temporal course of shape change is considerably advanced.

The results demonstrate that a possible role for differential LGN growth in ocular segregation cannot be ruled out. 


\section{Acknowledgements.}

First and foremost, a sincere thank you goes to Glen Jeffery, my supervisor, who gave me considerable freedom to pursue the work presented in this thesis, but kept me on the right tracks throughout.

My appreciation also extends to the numerous people whose paths I have crossed during my time at the Institute. From within the lab, these include Tim Pheby, Maria Ilia, and Claudia Thomas. From elsewhere, thanks must extend to all the staff in the animal house, who taught me how to handle a ferret properly (!) and to all the members of other labs who have made a drink on a Friday evening enjoyable - you know who you are!

A particular thank you goes to Christian Brechbüler at the Swiss Federal Institute of Technology in Zurich, who provided invaluable assistance when implementing the 3D modelling technique. The numerous discussions we had via e-mail provided me with a greater insight into the method he devised, which I would not have gained simply by reading.

Finally, my gratitude extends to my family, especially my mother, and to Ezi, and her family for all the support and encouragement you have provided throughout the past few years. Diolch yn fawr iawn i chi gyd.

My sincere apologies if I've left anybody out ! 


\section{Abbreviations.}

LGN lateral geniculate nucleus (dorsal)

CNS central nervous system

PGN perigeniculate nucleus

MIN medial interlaminar nucleus

SC superior colliculus 


\section{Contents.}

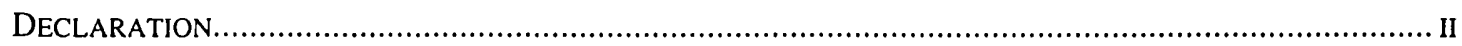

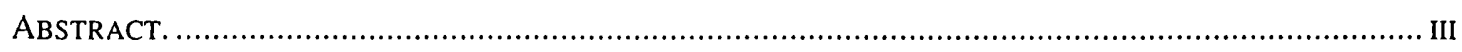

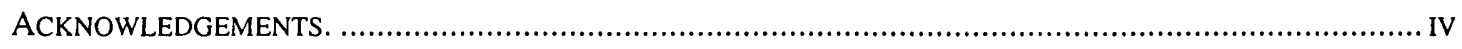

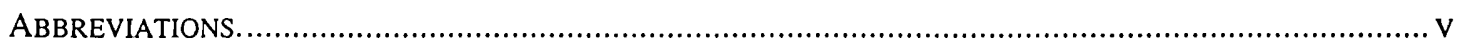

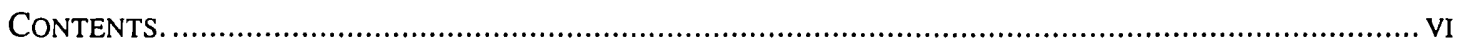

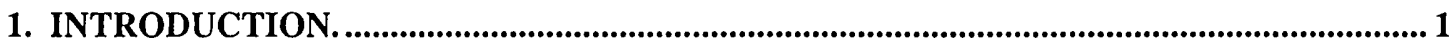

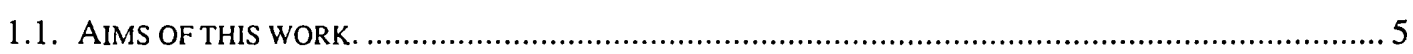

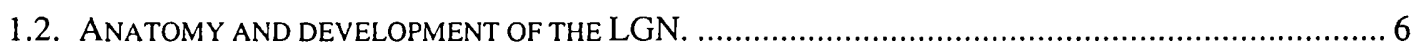

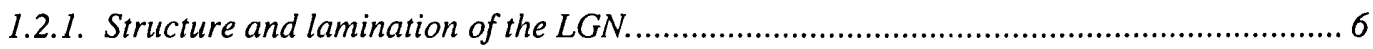

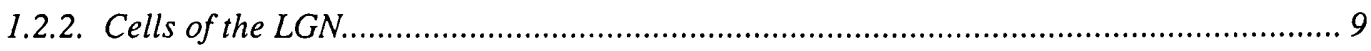

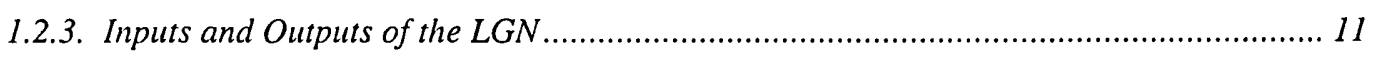

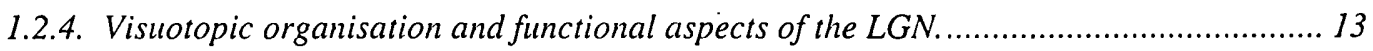

1.3. ThE FORMATION OF EYE-SPECIFIC LAMINAE...................................................... 14

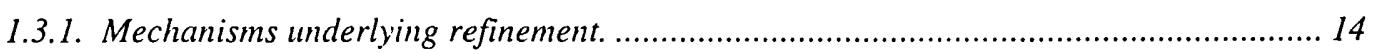

1.3.2. What determines the specificity of refinement ?................................................. 16

1.3.3. Facilitation of competitive interactions. ......................................................... 17

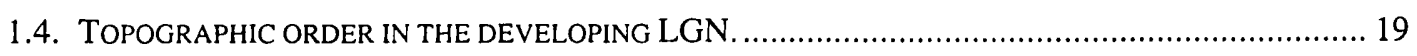

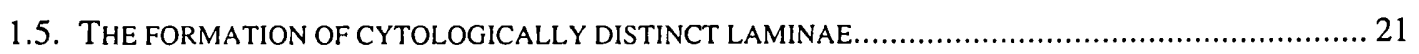

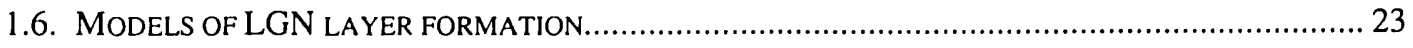

2. GENERAL MATERIALS \& METHODS................................................................ 25

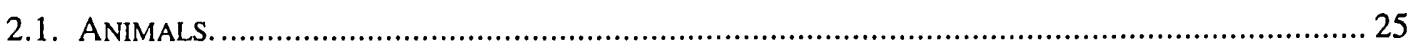

2.2. PreParation OF SECTIONS FOR CELLULAR STAINING................................................ 26

2.3. PreParation Of ANTERIOGRAdE LABELLED SECTIONS. ............................................. 27

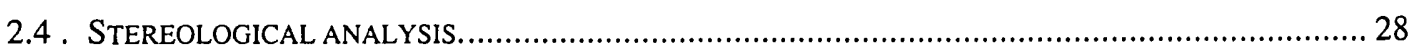

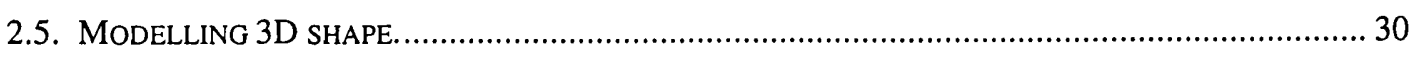

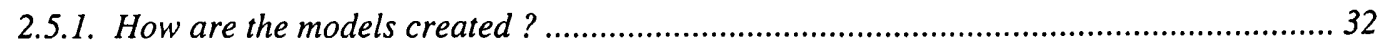

2.5.2. Modelling the shape of the ferret $L G N$............................................................ 35

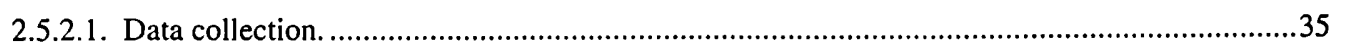

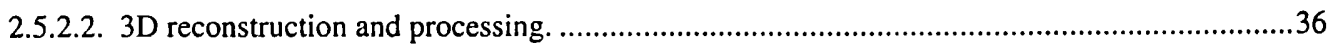

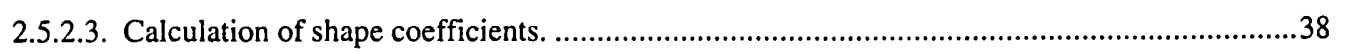

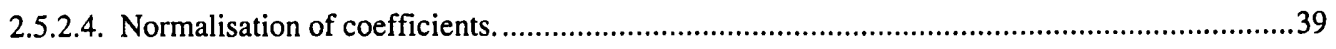

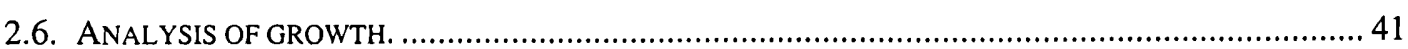

3. IPSILATERAL \& CONTRALATERAL PROJECTIONS TO THE DEVELOPING LGN. . 43

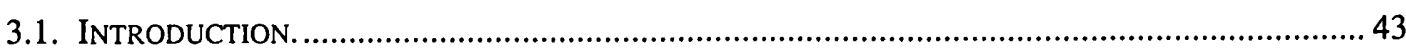

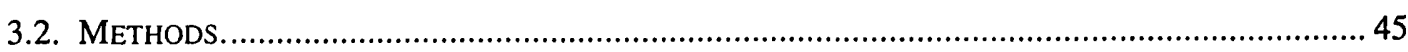


3.3. RESULTS. 45

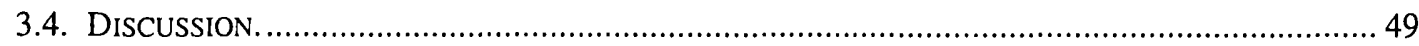

4. A CELLULAR ANALYSIS OF THE FERRET LGN. ...........................................51

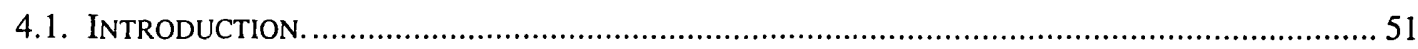

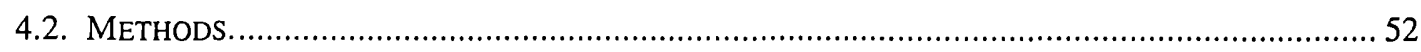

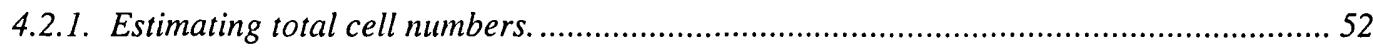

4.2.2. Spatial distributions of cellular densities.............................................................. 53

4.2.3. Cell areas................................................................................................ 53

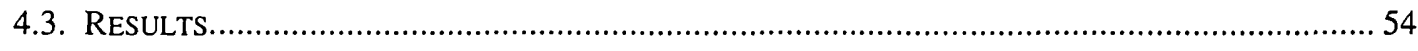

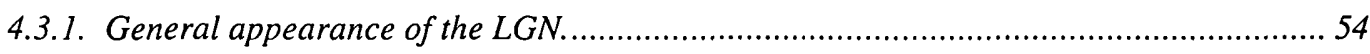

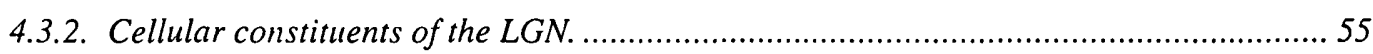

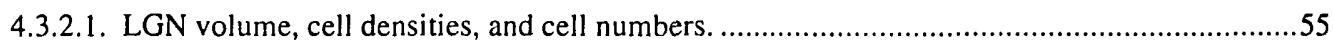

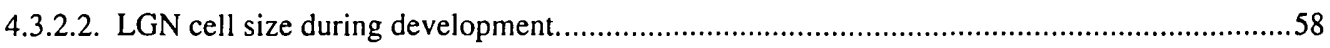

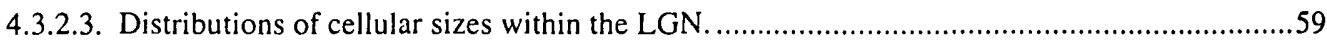

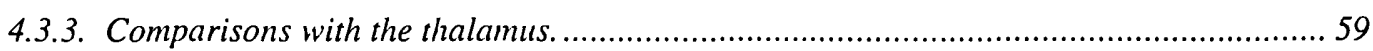

4.3.3.1. Cell densities in LGN and thalamus - measurements from mid LGN..................................59

4.3.3.2. Measurements from further dorsal and ventral regions..................................................69

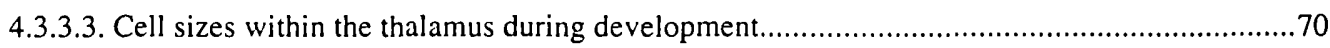

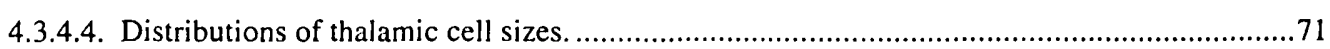

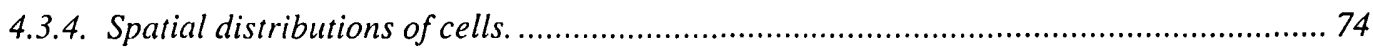

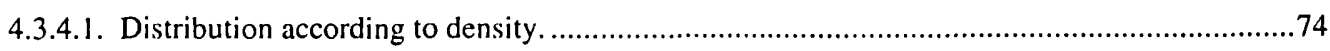

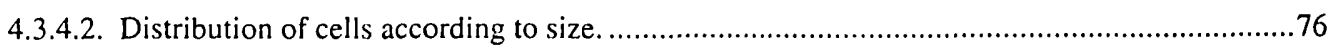

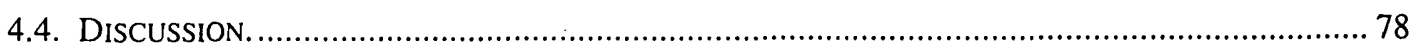

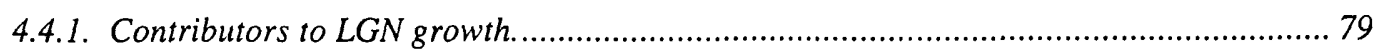

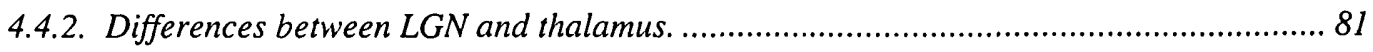

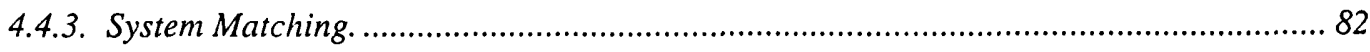

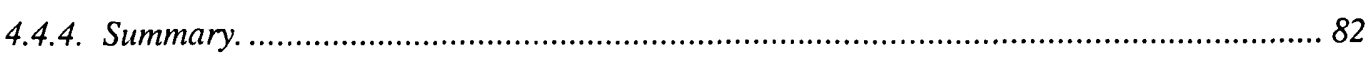

5. THREE-DIMENSIONAL GROWTH DYNAMICS IN THE FERRET LGN.....................84

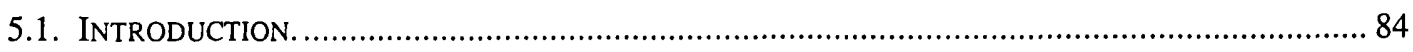

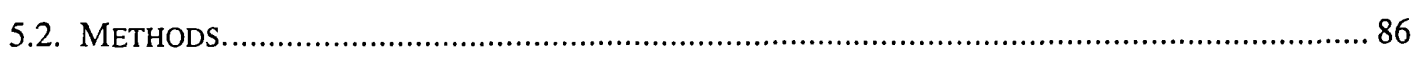

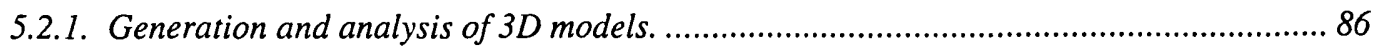

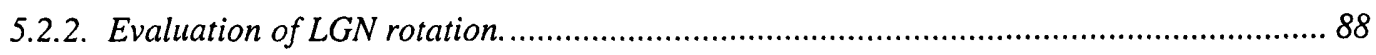

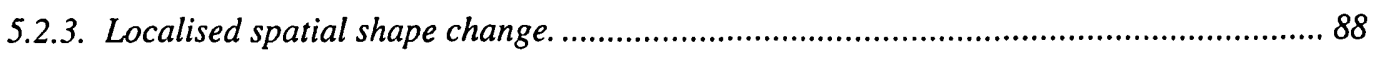

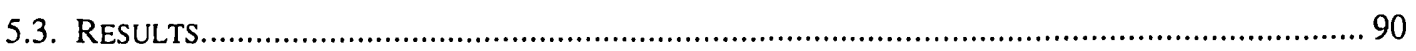

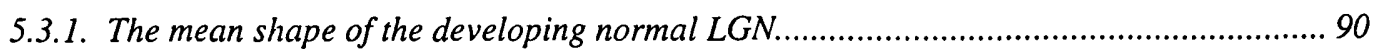

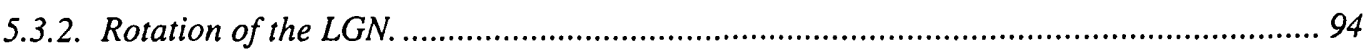

5.3.3. A time course for the changes in LGN shape. ................................................... 101 
5.3.4. Temporal relationships between measured LGN parameters. 102

5.3.5. What influences changes in shape ?..................................................................... 102

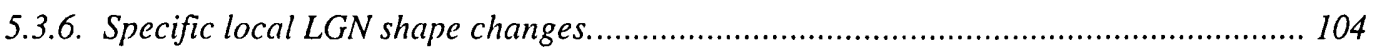

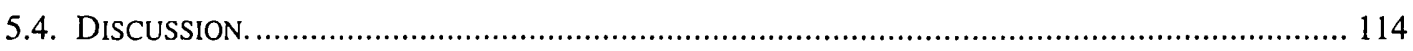

5.4.1. Temporal correspondences during development ................................................. 114

5.4.2. Specific shape changes during development ........................................................ 116

6. MODELS OF THE DEVELOPING IPSILATERAL \& CONTRALATERAL PROJECTION

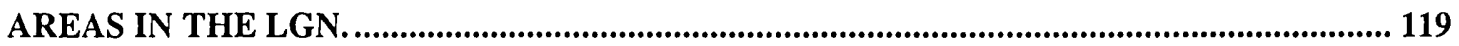

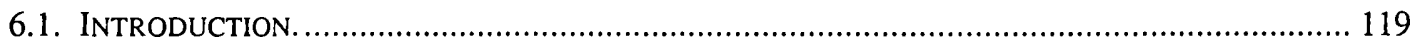

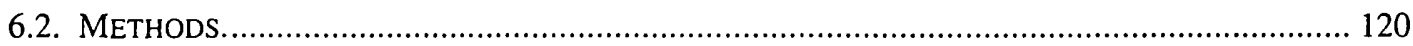

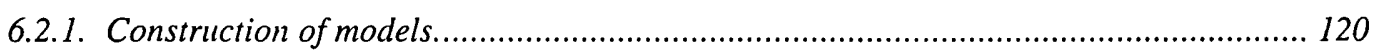

6.2.2. Calculation of transformation matrices describing global shape change. ................... 121

6.2.3. Verification of transformation accuracy.............................................................. 122

6.2.4. Investigating relationships between LGN models and terminal field models. ............. 123

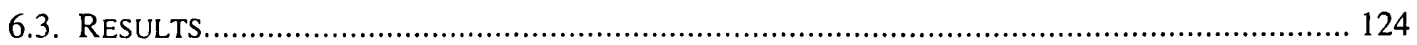

6.3.1. Mean models of the developing terminal fields. ...................................................... 124

6.3.2. Analysis of the relationship between changing $L G N$ structure and the development of

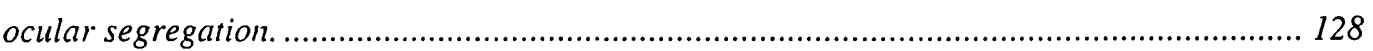

6.3.2.1. Evaluation of the accuracy of the transformation matrices.................................................. 128

6.3.2.2. Transformations of the LGN, and how they effect changes in their respective terminal field

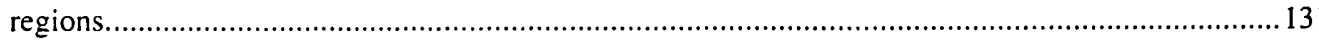

6.3.2.3. Further detailed transformations of the LGN, and how they effect changes in the terminal field

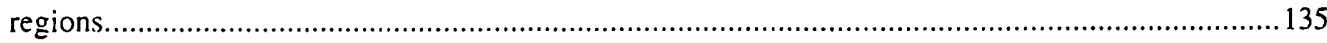

6.3.3. Spatial relationships between changing LGN structure and ocular distinct terminal field

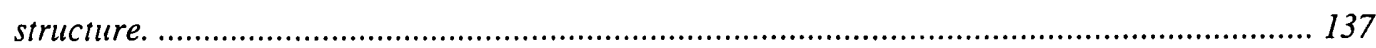

6.3.3.1. Relationship between specific changes in LGN shape and the corresponding terminal field region ipsilateral to the injected eye.

6.3.3.2. Relationship between specific changes in LGN shape and the corresponding terminal field region contralateral to the injected eye.

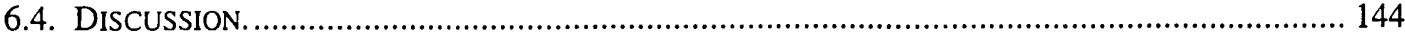

6.4.1. Do changes in LGN shape affect changes in terminal field regions?......................... 144

6.4.2. Relationships between specific LGN shape change and terminal field changes.......... 145

\section{THE ROLE OF RETINAL AFFERENTS IN REGULATING CELLULAR}

7.1. INTRODUCTION.

7.2. METHODS 149

7.3. RESULTS. 
7.3.1. The developing $L G N$ in horizontal cross-section.................................................... 150

7.3.2. LGN volume, cell numbers and densities, ............................................................ 152

7.3.3. Cell size in the monocularly enucleated ferret LGN.............................................. 154

7.3.4. Contributors to LGN growth in the enucleate........................................................ 157

7.3.5. LGN shape changes ipsilateral and contralateral to an enucleation......................... 158

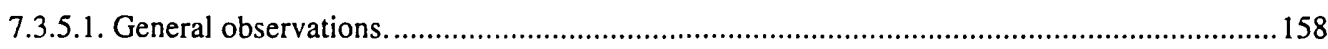

7.3.5.2. Temporal changes in shape

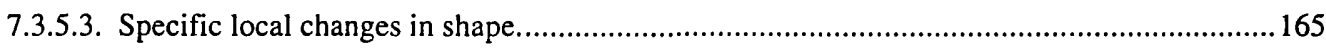

7.3.6. Temporal sequences of events - comparisons with the normal animal....................... 172

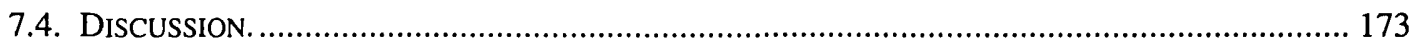

7.4.1. Cellular constituents of the enucleated animal. ....................................................... 173

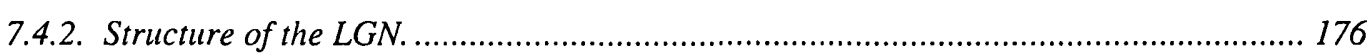

8. SUMMARY \& CONCLUSIONS. ............................................................................................. 178

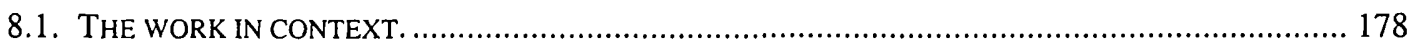

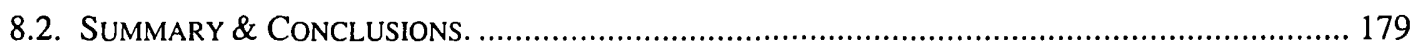

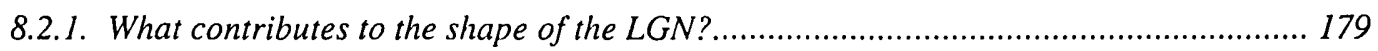

8.2.2. Does shape change contribute to ocular segregation? ............................................... 182

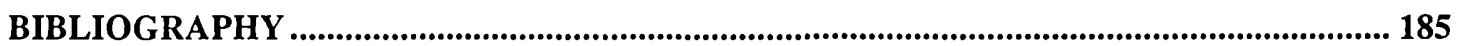

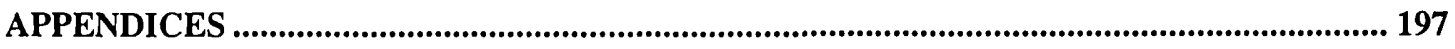

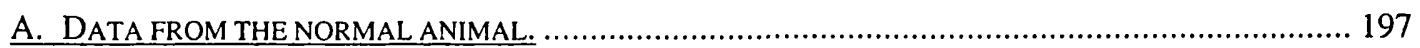

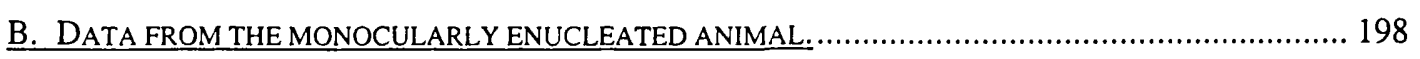

C. VALIDITY OF THE MODEL REPRESENTATIONS, ..................................................................... 200 


\section{Introduction.}

The brain, and more specifically, the thalamus, is a complex three-dimensional structure composed of numerous anatomical subregions. The thalamus (from the Greek $\theta \alpha \lambda \alpha \mu o \varsigma$, meaning inner chamber), is part of the diencephalon, and is an oval nuclear mass lying either side the third ventricle. It is, amongst other things, a relay station of the sensory pathways ending in the cerebral cortex. In the mammalian visual system, the most important thalamic nucleus subserving visual function is the dorsal lateral geniculate nucleus (LGN, Figure 1.1).

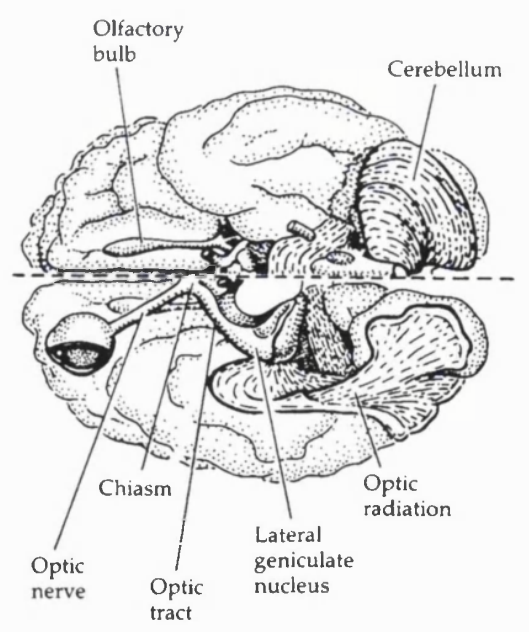

Figure 1.1 - The visual pathway in a partially dissected human brain as seen from below, showing the location of the LGN.

The nucleus displays a very specific pattern of connectivity and geometric structure. During the early part of this century, Minkowski $(1913 ; 1920)$ showed that 
axons innervating the LGN of the primate were organised into eye-specific layers. The findings were based on the fact that neuronal degeneration was observed in the nucleus following the removal of one eye. It is now known that lamination is a common feature in mammals with frontally placed eyes. It is due to the fact that mammals possessing a binocular field have a proportion of axons from the temporal retina that do not decussate at the optic chiasm but project ipsilaterally. This results in projections from both eyes being present in each nucleus, and arranged by some mechanism into eye specific layers. More recently, Rakic (1976) demonstrated that during development, projections from each eye were initially intermixed within the LGN, thus demonstrating that the refinement of early connections is necessary prior to the development of the normal adult pattern.

In addition to the arrangement of projections according to ocular origin, there are two other key features of LGN organisation; within each layer, there is topographic continuity of visual field representation. Second, the retinotopic organisation within each layer is aligned, such that a point in the visual field is represented by a line through the layers (Kaas et al., 1972). Unlike the organisation according to eye of origin, where initial connections are inappropriate and refinement is required, studies have shown that a crude topographic order exists very early in development, prior to eye-specific segregation (Jeffery, 1985; 1989).

The factors that regulate the development of these patterns have been the subject of numerous studies. However, research into these issues has concentrated on cellular and molecular events (see below), with relatively little attention paid to developmental changes in the geometric configuration of neuronal regions and the factors associated with these spatial transformations. It is known that during development, there are 
marked changes in the shape of the LGN in a number of species (for review, see Jones, 1985). The changes are gradual, and can be identified during the period of refinement.

But the absence of quantitative descriptors of structural change has so far excluded any analysis on the effects of spatial change in such events. It is not known what role differential growth of the LGN might play in the refinement of this structure, despite the temporal correlation between the two events.

In the central nervous system (CNS), quantitative analysis of geometric and spatial changes has been sparse. An early pioneer of the field, Thompson (1917), illustrated the importance of understanding geometric aspects of the natural world in a quantitative manner. While he developed new techniques for evaluating changes in shape, his classic work also described how many of the sciences of the era were limited to descriptive and classificatory methods :

\footnotetext{
"The zoologist has scarce begun to dream of defining, in mathematical language, even the simpler organic forms. When he finds a simple geometrical construction, for instance in the honey-comb, he would fain refer it to physical instinct or design rather than to the operation of physical forces; .... In short he is deeply reluctant to compare the living with the dead, or to explain by geometry or dynamics the things which have their part in the mystery of life."
}

(From Thompson, 1917)

The science of quantitatively describing, analysing and interpreting shape, or shape change is known as morphometrics. With the advent of computer technology in the past decade, this field has developed immensely. An abundance of techniques are available for characterising the shapes of organisms, and the changes in shape that occur as a result of development, evolution, experimental manipulation, or pathology (e.g. Rohlf and Bookstein, 1990; Marcus et al., 1996). The methods available to describe form, may be divided into two distinct groups : 
1. those based on homologous landmarks identified on the boundary or within the internal structure of an object, and

2. those based on the outline or surface of the object (usually used due to the paucity of landmarks).

There is a substantial difference in the two approaches. Analysis methods based on homologous landmarks generally describe localised changes in shape, and provide a basis for a biological interpretation of those shape changes. This is because the landmarks are bound to biologically meaningful locations. In contrast, methods based on outline data tend to provide a complete mathematical description (or model) of the entire object shape. While the mathematical descriptors themselves are abstract and have no direct biological interpretation, they can be important when used in a comparative study. In addition, outline based methods provide a one-to-one mapping between the observed structure, and the quantities used to describe the structure.

To date, most studies which employ techniques that fall into the above categories have focused mainly on areas such as phylogeny and ontogeny (e.g. see Rohlf and Bookstein, 1990). In the CNS, studies which have explored the issues of shape have generally used mechanical models based on physical laws to ascertain how particular structures arise during development. For example, the convoluted growth of the cerebral cortex has been of particular interest for a number of years (e.g. Richman et al., 1975; Raghavan et al., 1997; Van Essen, 1997). While these studies can explain how the cortex attains a particular form (mechanical tensions of axons, constraints of the skull, etc.), they do not explore the consequences of changes in shape.

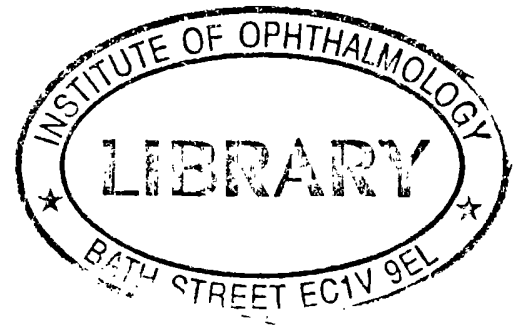




\subsection{Aims of this work.}

The work presented here seeks to explore the relationships between the formation of binocularly segregated retinotopic maps, and the differential growth of the nucleus. In addition to providing a quantitative description of three dimensional change in the structure of the LGN, both the causes of growth and the consequences of growth are investigated. The role played by cellular factors in regulating maturational changes in the structure of the LGN are examined. Cell numbers, density and size are estimated in the LGN, and compared in both spatial and temporal terms to the changing structure of the nucleus.

The hypothesis that differential growth in the LGN might contribute to binocular segregation is investigated by comparing the changes in LGN structure to those of the retinal ganglion cell terminal field structure, following monocular injections of anteriograde tracers. Both global transformations and local changes in shape are investigated to determine any relationships between the two events. In addition, the role of retinal afferents in regulating both the cellular constituents of the LGN, and the structure of the nucleus are explored by using monocularly enucleated animals.

In attempting to relate changes in the structure of the LGN with other events, one of the key aims of this work was to investigate and implement a method for quantifying shape and shape change. Models of the shape of the LGN at various developmental stages are created and employed in further analyses as described above.

The remainder of this chapter provides an overview of the anatomy and development of the LGN, which is followed by a review of recent work relating to the development of orderly connections within the nucleus. The final section provides a 
description of the small amount of work which has already been carried out on modelling the formation of layers in the LGN.

\subsection{Anatomy and development of the LGN.}

The LGN displays a varied but remarkably distinct recognisable structure in a number of different mammalian species. Three principal forms of the nucleus have been described in the literature: that of the rodent, carnivore and primate LGN (Jones, 1985). The carnivore LGN is given particular attention here, especially that of the ferret (see Section 2.1), and the following text focuses primarily on this order as it will be the experimental model used in this project.

\subsubsection{Structure and lamination of the LGN.}

The carnivore LGN has been described as having a characteristic pattern of structural features (Sanderson, 1974) which are evident in the ferret (Linden et al., 1981). In horizontal Nissl stained sections, the adult ferret LGN is situated on the lateral margin of the thalamus, adjacent to the optic tract. It is seen as a dark staining L-shaped structure having two limbs: a rostro-lateral longitudinal limb, roughly corresponding to the monocular segment of the nucleus and a medio-caudal transverse limb, corresponding to the binocular segment (Figure 1.2).

The nucleus has two well defined major laminae, $\mathrm{A}$ and $\mathrm{A} 1$, which receive contralateral and ipsilateral (crossed and uncrossed) afferents respectively. Layer A lies on the medial aspect of the nucleus, furthest from the optic tract, with layer A1 lying immediately lateral to it, occupying a central part of the medio-caudal longitudinal limb (the binocular segment). Between lamina A1 and the optic tract lie 
four smaller laminae: $\mathrm{C}, \mathrm{C} 1, \mathrm{C} 2$, and $\mathrm{C} 3$. Layers $\mathrm{C}$ and $\mathrm{C} 2$ receive contralateral inputs, while layer $\mathrm{C} 1$ receives ipsilateral inputs, and C 3 shows no clear evidence of any retinal input at all.
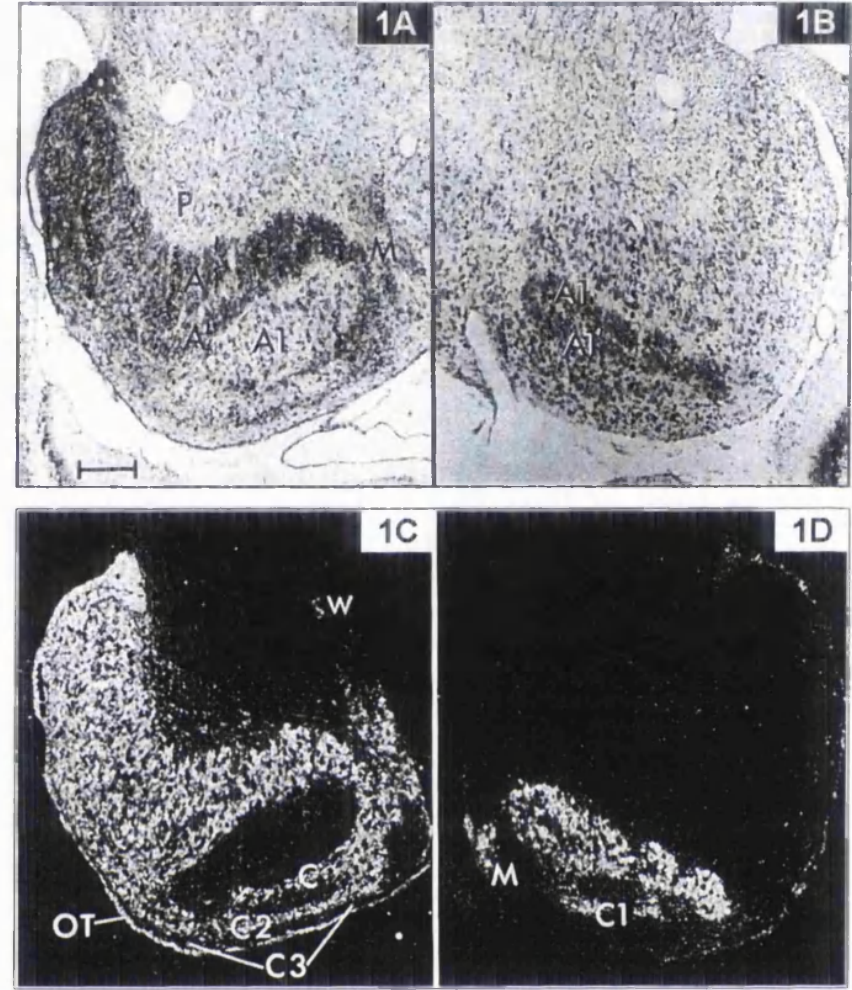

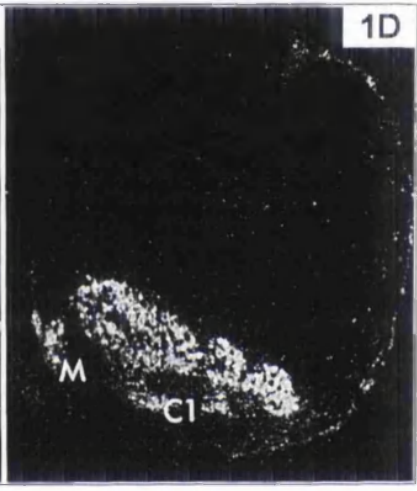

Schematic

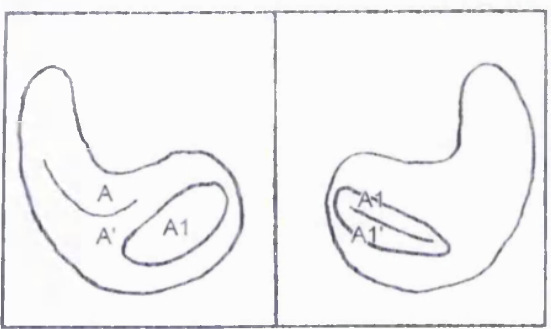

Figure 1.2 - Horizontal sections through the LGN of an adult ferret. The images show the laminar features of the nucleus as well as some related thalamic structures. A $\& A^{\prime}$ are the two leaflets of layer $A$, and $\mathrm{A} 1 \& \mathrm{~A} 1^{\prime}$ are the leaflets of layer Al. The C layers are all indicated,

as are the perigeniculate nucleus (P), the medial interlaminar nucleus (M), the geniculate wing (W), and the optic tract (OT). The schematic diagram on the right highlights the positions of the main $\mathrm{A}$ and $\mathrm{A} 1$ layers within the nucleus. Figure $\mathrm{IA}$ and $\mathrm{IC}$ show the same nucleus from the left hemisphere, and similarly, 1B and ID show the same nucleus from the opposite hemisphere. $1 \mathrm{~A}$ and $1 \mathrm{~B}$ are images taken under brightfield conditions, while $1 \mathrm{C}$ and $1 \mathrm{D}$ are taken under darkfield conditions. Rostral is towards the top, and medial is towards the centre of each pair of images. Scale bar: $250 \mu \mathrm{m}$. (From Linden et al., 1981).

Layer A and A1 comprise medium sized and large neurones. The cells of layer A1 are generally uniform in size throughout. Cells in layer A vary in size according to location: they are smaller at the anterior monocular tip, as well as in the dorsal regions of the nucleus (Linden et al., 1981). Between laminae A and A1 is a virtually cell-free interlaminar zone. Autoradiographs indicate that this region receives little if any retinal input. Sanchez-Vives et al (1996) have shown that the interneurons within the 
interlaminar zone possess attributes that are similar to neurones in the perigeniculate nucleus. This has led them to postulate that the interlaminar zones are actually part of the perigeniculate. In addition, both laminae A and A1 subdivide further into pairs of laminae referred to as leaflets. Electrophysiological studies have shown that these sublaminae consist of geniculate cells having either $\mathrm{ON}$ - or OFF-centre receptive fields (Stryker and Zahs, 1983).

Lateral to layer A1 is layer C, which extends into both the monocular and binocular segments of the LGN. It is easily recognised in the binocular segment due to its relatively large cells. Conversely however, it is difficult to recognise in the monocular segment since the cells are smaller. Further lateral to layer $\mathrm{C}$ is layer $\mathrm{C} 1$, which is made up of cells that are smaller than those in layer C. Similarly to layer A1, it does not extend into the monocular segment. Lateral again to $\mathrm{C} 1$ and nearer the optic tract is layer C2. It is a relatively thick layer of medium and small cells, and receives a well defined crossed retinal input. Similar to layer $\mathrm{C}$, it extends into the monocular segment. Layer C3 lies immediately adjacent to the optic tract, and is identified as a narrow, relatively unlabelled clear zone. Since many fibres must pass through layer C3 on their way to other layers, Linden et al (1981) have suggested that a "modest input" into this layer cannot be excluded.

In addition to the layers described above, two other thalamic nuclei have been regarded as associated with the geniculate. The medial interlaminar nucleus (MIN) lies medial to the caudal binocular limb of the LGN (Figure 1.2). In the mink, it has been shown to receive two distinct crossed retinal inputs (Guillery and Oberdorfer, 1977). Rostral and medial to layer A is the perigeniculate nucleus (PGN, Figure 1.2), which may be a subregion of the reticular nucleus (Cucchiaro et al., 1991; Uhlrich et al., 
1991). It consists of cells of mixed sizes, although contains none as large as those found in the geniculate. PGN axons innervate the A lamina of the LGN almost exclusively, and is itself innervated by axon collaterals of the geniculocortical and corticogeniculate pathways (Uhlrich et al., 1991).

\subsubsection{Cells of the LGN}

Neuronal cells may be classified according to their morphology or function. In accordance with Guillery's (1966) scheme for classification in cats, Sutton and Brunso-Bechtold (1991) assigned mature LGN ferret neurones to one of four classes based on their dendritic morphology, soma size, and shape (Figure 1.3). Class 1 cells may be divided into stellate and oriented subtypes. Class 1 stellate cells have large, multipolar somata, with disk-shaped dendritic fields, while class 1 oriented cells have large bipolar somata, with hourglass-shaped dendritic fields which are oriented perpendicular to laminar borders. Both subtypes can be characterised by their filiform appendages.

Class 2 cells may also be divided into the stellate and oriented subtypes. Class 2 stellate cells have medium, round somata with disk-shaped dendritic fields (usually with one primary dendrite larger than the others). Class 2 oriented cells have similar somata as their stellate counterparts, but have an octopus-shaped dendritic field. Class 2 cells display characteristic clusters of club-like dendritic appendages. Class 1 and 2 cells are found throughout the A layers, and in slightly reduced numbers in the $\mathrm{C}$ layers. Within the C layers, these cell classes are found in the magnocellular layers, although some may be found in the parvocellular layers. 
Class 3 cells have medium round somata, irregular dendritic fields with few branches, long stalked appendages, and are generally found in the A layers. Finally, class 4 cells have small oval somata, narrow dendritic fields oriented parallel to laminar borders, and filiform appendages. This class of cell is found exclusively in the parvocellular $\mathrm{C}$ layers.

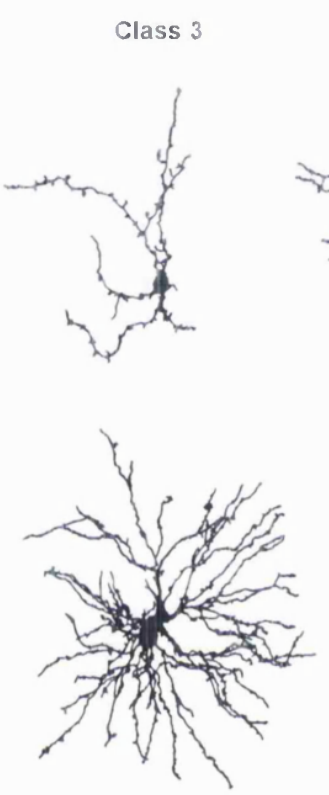

Class 2 - Stellate

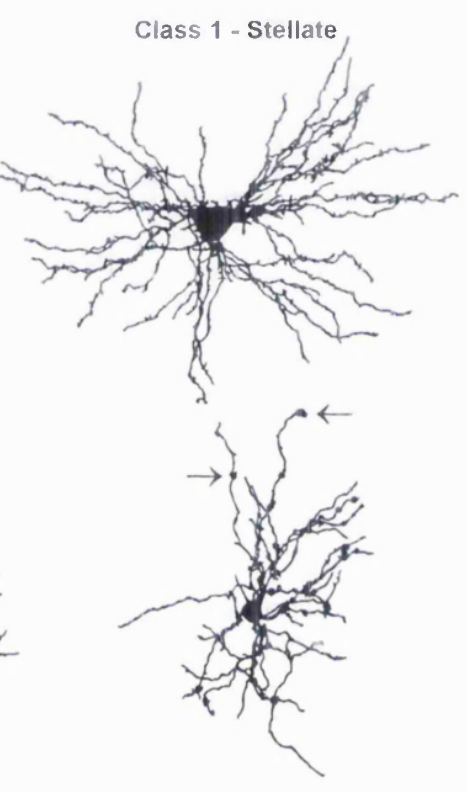

Class 2 - Oriented
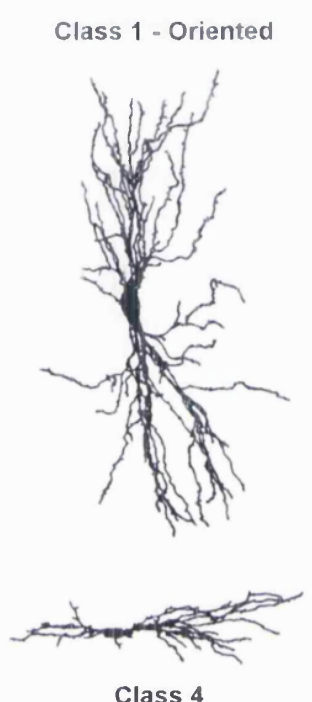

$100 \mu \mathrm{m}$

Figure 1.3 - Characteristic drawings of the cell classes in the mature ferret LGN. See text for detailed descriptions of the cells. The club-like appendages of the Class 2 cells are not overly clear in the drawings, but can be made out at the proximal branch points of the Class 2 Oriented cell (arrowed). (From Sutton and Brunso-Bechtold, 1991).

Functional cell classes in the ferret LGN have been reported by Price and Morgan (1987). They distinguished two types of LGN neurone which were classified as Xcells and Y-cells. The general difference between the two types is that X-cells respond well to stationary stimuli, and show linear spatial summation. Y-cells on the other hand show no clear spatial summation (i.e. a non-linear response) and respond better to changes in illumination or moving stimuli (Enroth-Cugell and Robson, 1966). Additionally, the axons of X-cells conduct more slowly than those of the Y-cells since 
they are generally smaller. Esguerra et al (1987) have also identified X-and Y-cells in the ferret LGN prior to filling them with horseradish peroxidase. The morphological similarities between the $\mathrm{X}$ - and $\mathrm{Y}$-cells described in this study, and the class 1 and 2 cells of Sutton and Brunso-Bechtold (1991) suggest some correlation between the morphological and functional classification of ferret LGN neurones. This is similar to that reported in the cat (Friedlander et al., 1979).

\subsubsection{Inputs and Outputs of the LGN}

The connections between visual nuclei in the mammalian brain are complex. As well as receiving afferents from the retina, the LGN receives inputs from the cortex, thalamic reticular nucleus, perigeniculate nucleus, superior colliculus, pretectum, and brain-stem reticular nucleus (Jones, 1985). However, retinal and cortical inputs are by far the largest, and are therefore the only ones considered here.

In the cat, approximately $75 \%$ of retinal ganglion cells project to the LGN (Illing and Wassle, 1981). Both X- and Y-cells project to laminae A and A1 and, in addition, Y-cells project to the magnocellular part of lamina $\mathrm{C}$ and the MIN. W-cells project mainly to the superior colliculus, but also innervate the parvocellular $\mathrm{C}$ layers of the LGN (Stone et al., 1979). But there are substantial differences in projections between species. For example, $90 \%$ of macaque monkey retinal ganglion cells project to the LGN (Rakic and Riley, 1983a), whereas only 35\% of the rat retinal ganglion cells do so (Martin, 1986).

Similar to the retinogeniculate input to the LGN, the other main pathway innervating the nucleus, the corticogeniculate projection, is also topographically organised. In the ferret, axons from area 17 terminate in most regions of the LGN, 
although there is a strong innervation focus within the interlaminar and interleaflet zones (Claps and Casagrande, 1990). Again, interspecies differences exist: area 17 of the cat projects with even distribution to all areas of the LGN (laminae and interlaminar areas). In addition, the cat has a projection from area 18 , which again terminates in all regions of the nucleus, but with greater density in the interlaminar zones, and an additional projection from area 19 which terminates in the $\mathrm{C}$ laminae (Updyke, 1975). Despite these differences, one common feature that exists is that the corticogeniculate projection is comprised of axons from layer VI modified pyramidal cells of the striate cortex (area 17).

In mammals, the visual cortex is the primary destination of LGN axons. In primates, LGN cells send their axons almost exclusively to the striate cortex, where they terminate primarily within granular layer IV (Garey and Powell, 1971). In cats, the axons of LGN relay cells project to extra-striate as well as striate areas. Both laminae $\mathrm{A}$ and $\mathrm{A} 1$ project to areas 17 and 18 , whereas the laminae of the C complex project to areas 17, 18, and 19, as well as several other areas (Garey and Powell, 1971). While the magnitudes of the projections are not the same, this anatomical arrangement suggests that the cat cortical visual areas are organised to receive parallel input from the LGN. This is in contrast to evidence which suggests that the standard organisation is for the LGN to project to one central area, striate cortex, from which information is disseminated in parallel (e.g. as in the primate). Variation from the norm in cat, and possibly other nocturnal predators (e.g. ferret), may reflect the need to facilitate rapid processing in relation to speed required for nocturnal predation (Casagrande and Norton, 1991). 


\subsubsection{Visuotopic organisation and functional aspects of the LGN.}

In most mammals, the LGN is made up of several layers, a number of which receive contralateral afferents, and a number of which receive ipsilateral afferents. Each layer represents either a complete half of the total visual field, or otherwise, a smaller binocular portion of the hemifield. The separate representations in each layer are aligned, and any point in the visual field corresponds to a line running perpendicular to the planes of the LGN laminae. These have been termed lines of projection (Bishop et al., 1962), and by extension of this, it has also been shown that an area in the visual field corresponds to a columnar volume in the LGN (Kaas et al., 1972).
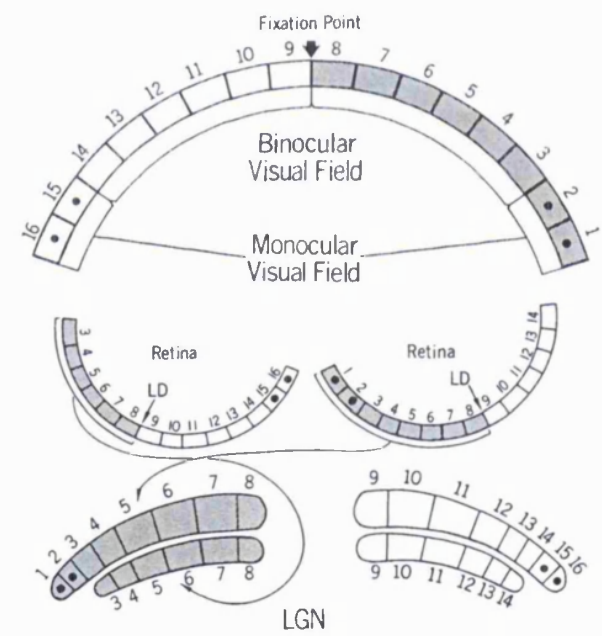

Figure 1.4 - Diagram showing the representation of the visual field on the retina and in the LGN. The geniculate shown is simplified, and contains only two layers, one receiving contralateral projections, and one receiving ipsilateral projections. Lines of projection are shown as the lines that separate the numbered segments in the schematic nucleus. $L D=$ line of decussation. (From Kaas et al., 1972).

The representation of the visual field in the LGN is topographical (Figure 1.4). Adjacent points in the visual field are represented by adjacent lines of projection. The naso-temporal division of each retina - known as the line of decussation - is represented by the plane joining the end points of ipsilateral and contralateral laminae. The temporal periphery of the contralateral visual field (i.e. the monocular field) is represented in the monocular segment of the LGN, a location where the layers of contralateral input extend beyond the layers of those receiving ipsilateral input. 
Between this location and the area representing the naso-temporal division is the representation of the remaining contralateral hemifield (i.e. the binocular field, Kaas et al., 1972)

\subsection{The formation of eye-specific laminae.}

Two processes are necessary for the development of normal visual function. First is the formation of connections between the retina and the LGN, where retinal ganglion cell axons have to grow and navigate to their thalamic target. Various hypothesis have been proposed as to the factors which influence axon guidance. These include chemical cues, mechanical guidance, and fibre-fibre interactions (reviewed in White and Chalupa, 1991). Second is the refinement of profuse connections, into the mature configuration. The following sub-sections introduce some of the work undertaken to comprehend the various processes involved in the separation of axon collaterals into eye-specific layers.

\subsubsection{Mechanisms underlying refinement.}

The biological mechanisms which bring about eye-specific laminae in the LGN have been established as being threefold (although these should not be considered as finite or exclusive):

- Overproduction and subsequent selective death of retinal ganglion cells

- Remodelling of retinal ganglion cell axon arbors

- Retraction of retinal ganglion cell axon collaterals

The first of these has been extensively investigated using a number of approaches. Rakic and Riley (1983a; 1983b) established that the optic nerve of the monkey contained over twice the number of axons around mid-gestation compared to that in 
the adult. Temporally, the period during which the loss of axons occurred was the same as the period during which the axons from both eyes became segregated in the LGN.

Other studies have provided more direct evidence for the role of ganglion cell death in the formation of laminar patterns. Jeffery (1984) employed a combination of both retrograde and anteriograde labelling to precisely quantify the number of retinal ganglion cells and volume of anteriogradely transported label in the LGN at progressive stages of development. The results demonstrated a high temporal correlation between a decline in the number of labelled retinal ganglion cells and the retraction of the terminal field.

The question of whether remodelling of axon arbors plays a role in laminar segregation has been addressed in studies which examined the changing morphology of individual retinal ganglion cell axons during relevant developmental periods. Sretavan and Shatz $(1984 ; 1986)$ used HRP to label fetal cat optic tract axons at various embryonic and postnatal stages. They reveal that during the period when axons from both eyes are intermixed within the LGN, they have a large number of side branches, combined with a simple terminal arbor. During a period coincident with eye-specific layer formation, there was a loss of the side branches, and an elaboration of the terminal arbor. This led to the conclusion that the combination of both these events led to a reduction in the amount of territory shared by both eyes, and hence the formation of eye-specific layers.

The final mechanism of retraction of axon collaterals has been substantiated by Ramoa et al (1989). The work illustrates how cat retinal ganglion cells of the $\beta$ class 
project initially to the superior colliculus (SC), whereas in the mature animal they terminate exclusively in the LGN. The use of retrograde labelling provided the necessary evidence to exclude the hypothesis that ganglion cell death was responsible for the disappearance of the projection from the $\mathrm{SC}$, due to the fact that labelled ganglion cells were still found after the period of cell death in the retina. Thus the only other explanation was that axon collaterals transiently innervated the SC, and subsequently retracted.

\subsubsection{What determines the specificity of refinement?}

The above has shown that the formation of eye-specific layers is a combination of various mechanisms, but the question remains as to which neurones should be the subject of such changes. The most popular hypothesis is that competitive interactions between axons from the two eyes takes place. This has been supported by various investigations, which have used enucleation techniques to determine what happens to the LGN in the absence of input from one or both eyes.

Rakic (1981) showed that when a single eye is removed before birth, the normal segregation of the primate LGN into six layers fails to occur, and a simple two-layered geniculate evolves, with a very ill-defined interlaminar zone. This author did note however, that the nature of the binocular interactions was selective, since the layers which evolved corresponded to the magnocellular and parvocellular regions of the normal animal. Furthermore, the work showed that visual experience is not a necessary requirement for binocular interactions, since the procedures were carried out before birth. 
A similar study performed in monocularly enucleated ferrets (Guillery et al., 1985) highlighted the fact that the size of the projection is also important in the development of a laminated geniculate. In the ferret, the contralateral projection is larger than the ipsilateral. The authors observed that a nucleus innervated contralaterally from the remaining eye resembled a relatively normal nucleus more than that innervated ipsilaterally, despite the fact that there is an increase in the number of axons in the ipsilateral pathway following the removal of one eye (Jeffery, 1984). The relative normality of the contralaterally innervated nucleus was characterised in terms of shape, size, and definition of laminae. Due to the removal of one eye, the ipsilaterally innervated laminae $\mathrm{A} 1$ and $\mathrm{C} 1$ were absent, but the space was occupied by contralateral afferents.

In addition to binocular interactions, another type of interaction prevalent in the developing LGN is that between the $\mathrm{X}$ - and Y-class of retinal ganglion cells. In a combination of anatomical and physiological studies in monocularly enucleated cats, Sherman and colleagues (Sherman, 1985; 1986a; Garraghty et al., 1986b) have shown that the arbors of the X-cells found in laminae $\mathrm{A}$ and $\mathrm{A} 1$, which are the first to develop, are initially exuberant. As development proceeds, the Y-axons expand their innervation of the geniculate, and force the competitive pruning of the X-cells' arbor such that they resemble their reduced adult form.

\subsubsection{Facilitation of competitive interactions.}

The early work of Hubel and Wiesel suggested a dependence on correlated patterns of neural activity from both eyes, for the normal development of the visual system (Hubel and Wiesel, 1965). Since then, tetrodotoxin (TTX), the $\mathrm{Na}^{+}$channel blocker, has been used to investigate the consequences of blocking activity on the 
visual system. The effects of blocking retinal ganglion cell action potentials by repeated intraocular injections of TTX into one eye of a new-born kitten was that there was abnormal segregation of retinal inputs onto LGN cells (Archer et al., 1982; Dubin et al., 1986). Shatz and colleagues have taken this further (Shatz and Stryker, 1988) by intracranial infusions of TTX in cat foetuses in utero over the period E42 to E56, when eye-specific layers appear. Comparison with a set of controls revealed that TTX severely disrupted the segregation of ganglion cell axons. However, injecting TTX into the brain not only affects the cells projecting to the LGN, but also the brain as a whole. Another investigation employed eye injections of TTX, and examined the resulting laminar patterns in the SC. The results revealed a much less marked effect on the disruption to segregation (Thompson and Holt, 1989).

If neural activity plays a role in segregation, then functional synaptic connections must be present in the retinogeniculate pathway. In addition, synapses must also be present in inappropriate regions of the geniculate. Serial section reconstructions from electron microscopic studies have confirmed this (Campbell and Shatz, 1992); contralateral axons established synaptic connections in inappropriate ipsilateral regions, and were later selectively eliminated during the formation of layers. Previous studies had already shown that prior to the period of segregation in the cat, functional synaptic transmission was present between ganglion cell axons and LGN neurones (Shatz and Kirkwood, 1984).

A point to note is that visual stimuli to the retina are not present during the formation of layers in the mammalian LGN (Shatz, 1994). It must therefore be assumed that the neural activity present in the retinogeniculate pathways is spontaneously generated rather than activity dependant. Galli et al (1988) 
demonstrated that such activity was present using extracellular microelectrode recordings from rat retinal ganglion cells in vivo. But, the presence of neural activity does not guarantee segregation, and a series of experiments by Stryker and Strickland (1984) demonstrated that the spatial and temporal pattern of activity was vital. Further experiments on the nature of the spontaneous ganglion cell activity have been carried out in the ferret (Meister et al., 1991; Wong et al., 1993), and demonstrate that the activity is correlated, even to the extent that neighbouring cells are more highly correlated than distant cells. A spatio-temporal analysis of data generated in these experiments showed that activity sweeps across the retina in waves, followed by periods of inactivity, before another wave in another direction is initiated.

\subsection{Topographic order in the deveioping LGN.}

It has already been considered how the initially intermixed fibres from each eye might segregate into individual eye-specific laminae. In the fully developed stage, retinal fibres are also arranged according to a strict topographic order. Unlike the organisation according to ocular origin, many studies have shown that there is a topographic map evident very early in development, prior to the segregation into eyespecific layers. The degree of error in this developmentally early map seems to vary between animals, and a number of studies have investigated its extent.

In the ferret LGN, Jeffery $(1985 ; 1989)$ illustrates the accuracy of the topographic projection using a combination of retinal lesions, followed by anteriograde labelling. Small localised retinal lesions were made in a number of ferrets at various ages, followed by intraocular injections of HRP into the same eye. The pattern of labelling in the LGN (and SC) was such that the experiments showed that not only was the 
larger contralateral projection retinotopically organised, but in addition, so was the smaller ipsilateral projection. The studies do not expand on the degree of error which may be present in the projections, and it is reported that there is a small superficial region of the LGN in which a topographic map cannot be demonstrated, probably the C layers.

Chalupa (1998) demonstrated the degree of order in the ferret retinocollicular projection by placing focal deposits of 1,1'-dioctodecyl-3,3,3'3'-tetramethylinodocarbocyanine perchlorate (DiI) into the retina, and observing the pattern of anteriograde labelling. The results of this are in agreement with Jeffery $(1985 ; 1989)$, in that the developing ferret shows a remarkable degree of precision in early topographic projections. Moreover, this study highlights the order exhibited by the uncrossed pathway, and postulates that the infrequent innervation errors made by axons from inappropriate areas of the retina reflect decussation errors rather than topographical errors.

In comparison to the carnivore, the extent of topographic errors in the rat seems greater (O'Leary et al., 1986; Yhip and Kirby, 1990). These studies employed retrograde labelling of either the caudal or rostral aspects of the SC, and provide quantitative measures of topographic errors based on the number of labelled cells observed in the retina. In animals injected on the day of birth, O'Leary and colleagues (1986) reported that approximately $14 \%$ of the labelled ganglion cells in the contralateral retina made topographic errors, while Yhip and Kirby (1990), in animals 2 days older, reported a similar figure of approximately $11 \%$. 
Similar to the formation of eye-specific layers, the mechanisms which refine the crude topographic map are probably numerous. O'Leary et al (1986) have shown that ganglion cell death plays a role in the process: observation of labelled ganglion cells at different time periods, following an injection into localised regions of the SC, showed that misprojecting cells were lost. The elimination process also coincided with the period of naturally occurring ganglion cell death. Chalupa and Snider (1998) proposed that directed axon ingrowth could play a role in ordering of the carnivore retinocollicular projection. However, Simon and O'Leary (1992) illustrated that this mechanism for establishing topographic order is inadequate in the rat, and proposed that molecular mechanisms which control the elimination of inappropriate axons and promote the arborization of correctly positioned axons play a significant role in the development of the retinotopic map. Shatz has suggested that the correlated activity of retinal ganglion cells (Meister et al., 1991; Wong et al., 1993) could help in refining the topographic map in the LGN (Shatz, 1994). Finally, Jeffery $(1989 ; 1990)$ proposed that a shifting of maps brings about the adult pattern: the arrangement in the neonate ferret may be thought of as two overlapping retinotopic maps, which shift in relation to each other, such that the mature projection appears.

\subsection{The formation of cytologically distinct laminae.}

The above sections have highlighted data available on the developmental changes occurring during ocular and topographic organisation in the LGN. In contrast, very little is known about the cellular and extracellular environment of the LGN during this period. In the ferret, Linden et al (1981) described a homogenous distribution of cell bodies in the immature nucleus, which develop into the characteristic cytoarchitectural layers of the adult. Retinal ganglion cell axons begin their segregation before any 
cellular layers become apparent, but clear cytoarchitectonic laminae appear before binocular segregation is complete (Linden et al., 1981). This lead to the conclusion that there must be some independence between the development of the two forms of lamination, i.e. ocular and cytoarchitectonic

Hutchins and Casagrande (1990) employed immunohistochemical staining of glial cell processes in order to study their relationships with retinal afferents and the cellular structure of the LGN. Glial cell processes were visualised with antibodies to glial fibrillary acidic protein (GFAP) in both ferrets and tree shrews, and the results suggested their involvement in cytoarchitectonic layer formation. In the ferret, the authors stated that glial cell lamination begins during a period when segregation of retinal afferents is almost complete. As neuronal cell lamination begins to appear, positive bands of GFAP can be seen in the developing interlaminar zones. As development proceeds, the GFAP+ processes become clearer, and can also be observed in the areas distinguishing between the leaflets of layers A and A1. By adulthood, a considerable change is seen in the pattern of label, where glial processes are more or less random throughout the LGN.

Another study emphasised the role which glia may play in the development of cytologically distinct LGN laminae. A keratan sulfate proteoglycan, ABAKAN, apparent on the surface of astrocytes, and identified by the antibody TED15, shows similar labelling to that of GFAP (Robson and Geisert, 1994). The authors suggested that the expression of ABAKAN in the interlaminar zones could be as a barrier for innervating axons and dendritic processes, and later in maintaining the division between ON and OFF axons from the same eye. 
The role of the extracellular matrix in the development of the ferret LGN has also been investigated. The extracellular matrix comprises of a number of components, some of which have been implicated in the process of cell migration (Sanes, 1989). Brunso-Bechtold et al (1992) examined the distribution of fibronectin (a component of the extracellular matrix) in the developing LGN, and provided similar results to Hutchins et al (1990). Fibronectin was localised in the interlaminar zone between layers A and A1, followed by stronger label later in development capable of distinguishing the ON and OFF sub-laminae of these layers. In contrast to the random distribution of GFAP, fibronectin is no longer present in the adult geniculate.

\subsection{Models of LGN layer formation.}

Given the wealth of work undertaken on the biological aspects of the development of the LGN, relatively little has been done in terms of modelling specific processes. A connectionist approach to modelling ocular segregation and topographic order in the nucleus was adopted by Keesing et al (1993). The architecture of the neural network model was very simple: the LGN was a two-dimensional structure 10 cells wide and 8 cells deep, while the ipsilateral and contralateral retinae were one-dimensional structures of 50 cells each. Input to the network at the retinal layer comprised of simulated retinal waves. Connections between retina and LGN cells were then strengthened or weakened according to a correlational Hebbian rule (i.e. when preand post-synaptic activity are sufficiently correlated, connections are strengthened; Hebb, 1949). Simulations produced results which demonstrated the formation of ocularly distinct layers and topographic order, and were consistent with observations previously made in the cat. Another study extended this by investigating other key features of the model, in particular the nature of the retinal inputs (Eglen, 1995). 
Analyses concerning LGN structure have focused on the transition from six to four layers as observed in the rhesus monkey's LGN. The reasons for such a transition have been the subject of two investigations (Lee and Malpeli, 1994; Tzonev et al., In press). Models from both studies (the former in 2D, and the latter in 3D) supported the hypothesis that small laminar gaps, corresponding to the position of the blind spot in each eye, introduce a singularity into the system which guides the development of LGN morphogenesis. The point at which the singularity (or gap) occurs corresponds to the transitional point from six to four layers. The implication of both studies is that changes to biological structures could be affected by very small simple anomalies.

This thesis aims to model the complete external structure of the LGN at various stages of development employing new shape modelling methods. The models will then be employed in further analyses to determine whether changes to the shape of the LGN influence the formation of binocularly segregated retinotopic maps. 


\section{General Materials \& Methods.}

\subsection{Animals.}

The work described here is based on tissue obtained from the pigmented European ferret (Mustela putorius furo). The ferret was the animal model of choice for a number of reasons. The animal is easy to breed and can have large litters. More importantly, it is a member of the order Carnivora, and, similar to other members of this order, has a laminated LGN. In addition, its visual pathway is very similar to that of the cat, whose visual system has been well documented. Finally, young ferrets are born at a stage when their visual system is relatively immature, 30 days before eye opening, and prior to LGN lamination and ocular segregation. This provides an excellent opportunity to study issues related to the development of the LGN.

A summary of the number of animals used and their respective age groups is provided in Table 2.1. Animals aged P36 (postnatal day 36) and under were bred at the Institute of Ophthalmology. All kits were from jills mated with the same male, all of which showed the characteristic 'mask' of the pigmented ferret (Figure 2.1). Those animals aged greater than P36 were obtained from the Department of Anatomy, University of Oxford, all of which had fully pigmented coats and eyes. 


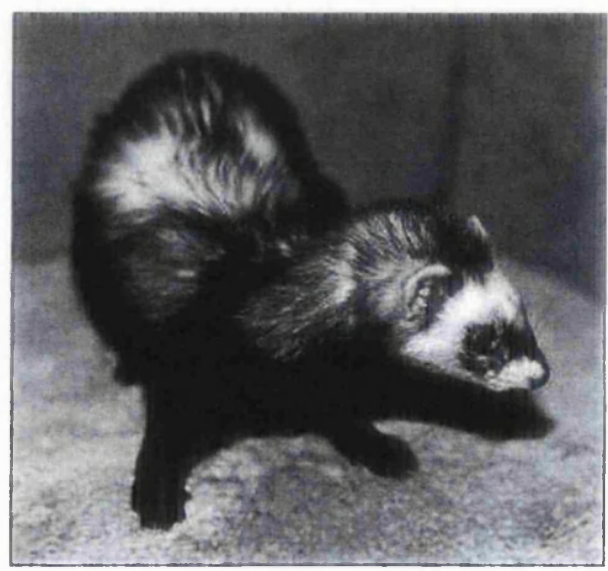

Figure 2.1 - A typical example of the pigmented European ferret (Mustela putorius furo), illustrating its characteristic coat and mask.

The brains of monocularly enucleated ferrets (Mustela putorius furo) were obtained from Dr B. Reese at the Neuroscience Research Institute, University of California, Santa Barbara. The number of animals used, and their different age groups are also shown in Table 2.1.

\begin{tabular}{|c|c|c|c|c|c|c|c|c|c|c|c|c|c|}
\hline Age & E38 & P0 & P2 & P5 & P8 & $\mathrm{P} 10$ & P23 & P25 & P36 & P47 & P83 & P111 & P140 \\
\hline Norm. & 2 & 5 & 2 & 4 & 4 & 4 & 1 & - & 2 & 2 & 3 & 3 & 3 \\
\hline Enuc. & - & - & - & 2 & - & 3 & - & 2 & - & 2 & - & - & - \\
\hline
\end{tabular}

Table 2.1 - A summary of the normal (norm.) and monocularly enucleated (enuc.) animals used in the study. E38 was selected as the first date since it is known that at this stage the nucleus is immature both in terms of fibre organisation and shape, while at P140, the nucleus is completely mature (Linden et al., 1981). Greater numbers were included in groups between E38 and P10 since this is the period of greater change. Dates of enucleations were such that they evenly spanned the range of the normal animals.

\subsection{Preparation of sections for cellular staining.}

Surgical procedures for monocular enucleation were as follows: newborn ferrets were anaesthetised with methoxyflurane (Metofane), the lids were opened, and the left eye was enucleated intact using iridectomy scissors. Gelfoam was inserted into the orbital cavity, and the lids then reapposed using cyanoacrylate. Animals were allowed to recover in an incubator at $37^{\circ} \mathrm{C}$, and then returned to the jill. 
All animals were killed with a lethal injection of sodium pentobarbitone (Euthatal, $60 \mathrm{mg} / \mathrm{kg}$ ), and perfused through the heart with $0.1 \mathrm{M}$ phosphate buffer saline followed by $3 \%$ paraformaldehyde fixative ( $\mathrm{pH} 7.2$ ). All perfusions were carried out by $\mathrm{Dr}$ Glen Jeffery at the Institute of Ophthalmology. Brains were either removed immediately and stored in fixative, or the brain was exposed in the skull, and the head stored in fixative.

Prior to sectioning, brains were trimmed, and dehydrated in a graded series of alcohols $(50 \%, 75 \%, 95 \%, 3$ changes $100 \%)$ for half to one hour each. Trimming involved dissecting the brain along the midline, and in the case of older animals, removing the cortex to aid resin penetration during embedding. The tissue was embedded in glycolmethacrylate (Historesin ${ }^{\mathrm{TM}}$, Leica $\mathrm{GmbH}$ ), and serial horizontal sections were cut at $30 \mu \mathrm{m}$ on a rotary microtome using a tungsten-carbide knife. All sections were collected, mounted on clean glass slides, and baked on a hotplate at 70$80^{\circ} \mathrm{C}$ for 24 hours. Sections were stained with Giemsa for $1 \frac{1 / 2-2}{2}$ hours before dehydrating, clearing in a series of alcohols, and coverslipping.

\subsection{Preparation of anteriograde labelled sections.}

Ferrets at P5, P8, and P12 were anaesthetised with $1.5 \mathrm{ml} / \mathrm{kg}$ of saffan, whereas P0 ferrets which were anaesthetised by cooling. Unilateral intraocular injections of a $30 \%$ horseradish peroxidase (HRP, Sigma) solution in $1 \%$ dimethyl sulphoxide were made, and delivered manually with a $10 \mu 1$ Hamilton syringe by Dr Glen Jeffery. The amount of HRP solution injected varied according to the age of the animal, but was generally between $3 \mu l$ and $5 \mu l$.

Following a 24 hour survival, animals were killed with sodium pentobarbitone (Euthatal, $60 \mathrm{mg} / \mathrm{kg}$ ), and perfused through the heart with $0.1 \mathrm{M}$ phosphate buffer 
saline followed by $2 \%$ paraformaldehyde and $2 \%$ gluteraldehyde in $0.1 \mathrm{M}$ phosphate buffer ( $\mathrm{pH}$ 7.2-7.6). Brains were immediately removed and placed in fixative for a further 2 hours at $4^{\circ} \mathrm{C}$. They were then washed in $0.1 \mathrm{M}$ phosphate buffer, placed in a $25 \%$ sucrose solution (in $0.1 \mathrm{M}$ phosphate buffer), and stored overnight at $4^{\circ} \mathrm{C}$.

Serial horizontal $50 \mu \mathrm{m}$ thick frozen sections of the brain were collected using a sliding microtome, and transferred into $0.1 \mathrm{M}$ phosphate buffer. For the detection of HRP, sections were processed using the TMB-AHM method (Olucha et al., 1985) which uses tetramethyl benzidine (TMB) as a chromogen and amonium heptamolybdate (AHM) as a stabilising agent. Sections were mounted on gelatinised slides, and air-dried overnight before clearing in xylene and coverslipping.

\section{4 . Stereological analysis.}

The stereological technique of estimating total cell number $(N)$ is a two stage task. The first is the calculation of the reference volume $\left(V_{\text {ref }}\right)$, and the second, the estimation of the numerical density $\left(N_{v}\right)$ of cells. The total cell number may then be calculated as the product of the reference volume and the numerical density (West and Gundersen, 1990) :

$$
N=V_{r e f} \times N_{v}
$$

These individual quantities that comprise the total number of cells are unbiased estimators, and combined with systematic random sampling, they approach the true values of the parameters.

Calculation of the volume of the LGN was performed with a combination of two techniques, the Cavalieri estimator, and point counting: 
- Cavalieri (Cavelieri, 1966) stated that an unbiased estimate of the volume of a sectioned object could be obtained from the product of the sum of the areas of the individual sections, and the distance between them.

- In addition, unbiased estimates of the section areas can be made by overlaying the sections with a lattice of points, and counting the number of points which fall within the region of interest.

Sections were placed under a microscope equipped with a video camera connected to a stereological image analysis system (Kinetic Imaging, UK). The software superimposed a grid onto the field of view, and any points lying within the borders of the LGN were counted, and entered into the computer to automatically calculate $\mathrm{V}_{\text {ref. }}$ Individual sampling schemes (i.e. distance between sections, and grid point density) are summarised in the methods sections of relevant chapters. The schemes were chosen to minimise the amount of sampling required, but maximising the precision of estimates.

The numerical density $\left(\mathrm{N}_{v}\right)$ of LGN neurones was calculated using the optical dissector method (Braendgaard and Gundersen, 1986; Gundersen, 1986), and employing a systematic random sampling scheme. Formally, $\mathrm{N}_{\nu}$ is defined as :

$$
N_{v}=\frac{\bar{Q}^{-}}{h \times a} \quad \text { where }: \begin{aligned}
& \bar{Q}^{-} \text {is the mean number of cells per frame, } \\
& h \text { is the height (or depth) over which counts are made, and } \\
& a \text { is the area of the dissector frame. }
\end{aligned}
$$

The method utilises the capabilities of high numerical aperture lenses to view thick sections at varying depths. As the microscope is focused over a known depth through the section, cells are counted as they come into focus. The stereological software 
superimposed a suitable counting frame onto the field of view, and the vertical movement of the stage was measured with a digital depth gauge.

\subsection{Modelling 3D shape.}

The method employed here to characterise three-dimensional shape is that proposed by Brechbühler (1995). It is a method of fitting a closed surface (i.e. one that has no holes or handles) to a set of connected points in three-dimensional space, conceptually the same as fitting a curve to a set of points. The results are a series of equations (or functions) defining the fitted surface. Each equation defines the $x, y$, and $z$ component of a point on the surface, and are defined parametrically by $x(\theta, \phi), y(\theta, \phi)$, $z(\theta, \phi)$. The functions are expressed in terms of two angles $\theta$ and $\phi$, and these may be considered as latitude and longitude respectively. When the angles $\theta$ and $\phi$ lie in the range :

$$
\begin{gathered}
0 \leq \theta<\pi \\
0 \leq \phi<2 \pi
\end{gathered}
$$

the functions define points covering the entire surface of the object, thus providing a mathematical description. The reasons for expressing the surface shape in terms of two angles is that the initial representation of the shape (i.e. the set of connected points in $3 \mathrm{D}$ space) is mapped onto the surface of a sphere (where any point can be defined in terms of latitude and longitude). This mapping from three-dimensional space to the socalled parameter space, allows the distribution of points to be fitted with a set of functions which only exist on the surface of the sphere. The functions are known as spherical harmonic functions (Muller, 1966), and they provide a measure of the spatial frequency constituents of the surface structure (similar to the way a Fourier analysis expresses a waveform in terms of its various frequency components). 
Spherical harmonic functions may be considered as polynomial equations with an infinite number of terms, and the implication of this is that the equations have to be truncated at some point. Therefore, the greater the number of terms in the harmonic functions, the better the fit between the original data and the fitted surface. The number of terms retained in the equations relate to the degree or number of spherical harmonics. Figure 2.2 illustrates these concepts with a simple object fitted with spherical harmonic functions of varying degrees.

A

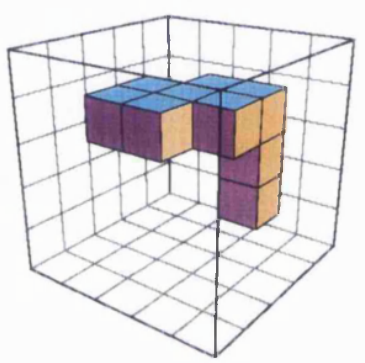

C

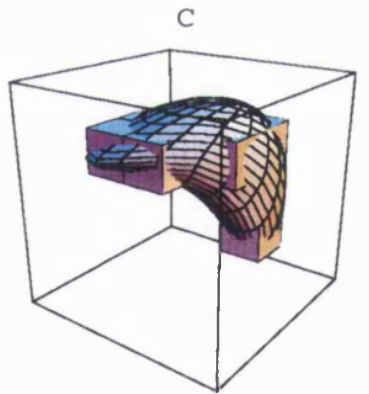

B

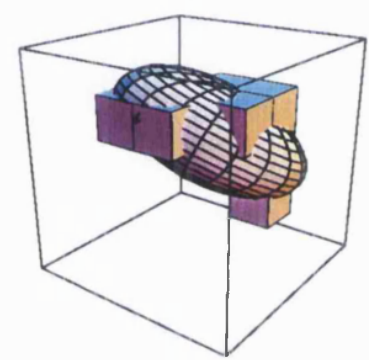

$\mathrm{D}$

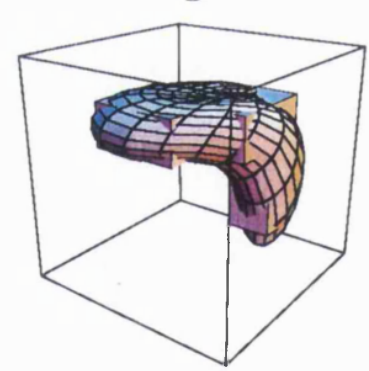

Figure 2.2 - A simple object in a $5 \times 5 \times 5$ voxel (the three dimensional equivalent of a pixel)

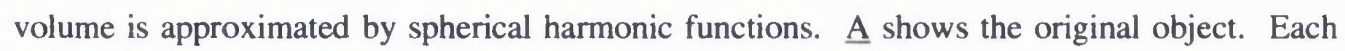
cube represents a single voxel, i.e. a point in $3 \mathrm{D}$ space. $\underline{B}, \underline{\mathrm{C}}$, and $\underline{\mathrm{D}}$ illustrate how the greater number of terms in the spherical harmonic functions describe a surface which better approximates the original object. The number of harmonics (i.e. the degree of harmonics) included in each series of $\underline{B}, \underline{C}$, and $\underline{D}$ are 1,2 and 3 respectively.

For modelling different surfaces, the equations will essentially be identical, only the coefficients of the parameters of the equations will differ. These coefficients can therefore be used as numerical representations of the shape being modelled, and may 
be subjected to conventional numerical analyses. An alternative is to consider the coefficients as the representation of the shape in a multi-dimensional space. So, for example, a shape described by 10 coefficients would represent a single point in a tendimensional space.

\subsubsection{How are the models created?}

The first step in modelling surface shape is to create the original representation of the shape to be modelled. Here, this will be a binary image in three dimensions. A binary image (in 2D) is one in which the pixels it comprises are either on or off (i.e. have value 1 or 0 ). By extension, a 3D binary image will be one where voxels are either present or not. A surface is then fitted as close as possible to the faces of those voxels defining the structures exterior surface.

When the shape data is in suitable format (as described above), the method of fitting a surface essentially comprises of the following three stages :

1. Mapping the exterior faces of each voxel in the 3D image onto the surface of a unit sphere. This stage is necessary as spherical harmonic functions are only defined on the surface of a sphere. The process can essentially be considered as converting the co-ordinates of each point of an object to latitude and longitude points. The final result is a unique mapping from the surface of the original object to the surface of a sphere. In addition, the mapping is continuous, i.e. neighbouring points in the original object will map to neighbouring points on the sphere. Figure 2.3 illustrates the mapping of a single face onto a sphere. 

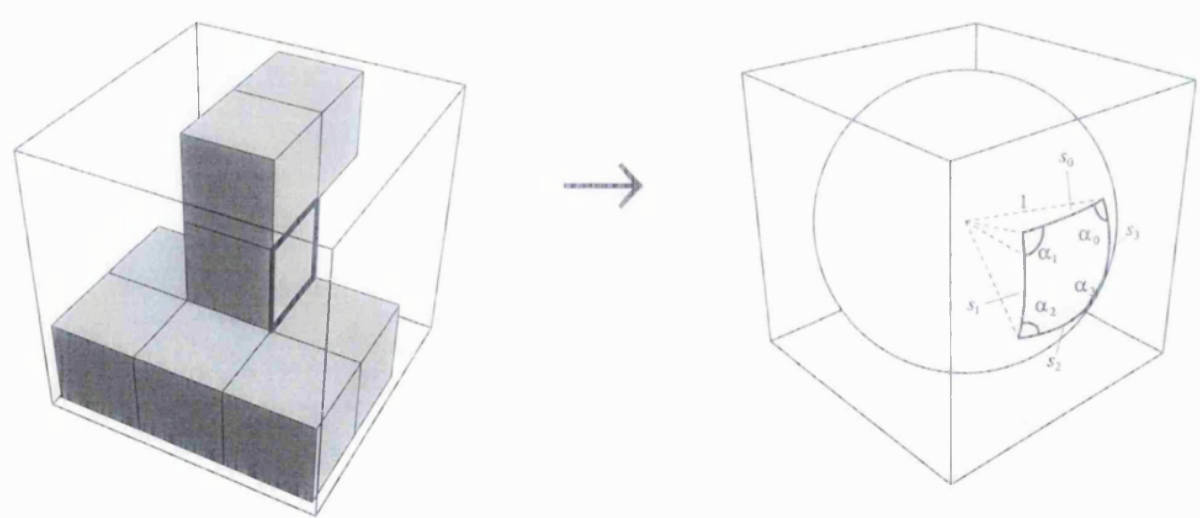

Figure 2.3 - Illustration of how a single face is mapped onto the surface of a unit sphere. The diagram illustrates a special case where the square face of the voxel is almost fully preserved on the surface of the sphere. In reality, this is never possible and distortions are introduced, while attempting to preserve the unique mapping (figure from Brechbühler, 1995).
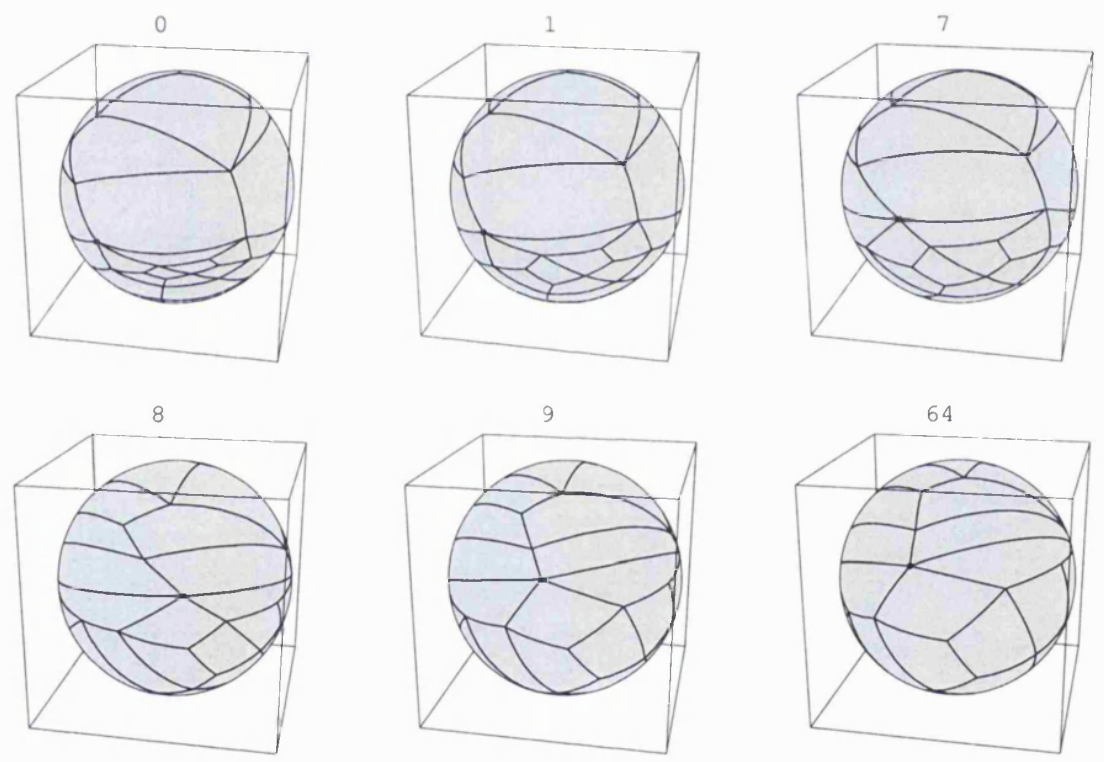

Figure 2.4 - Diagrams illustrating the distributions of points on the surface of the sphere following various iterations of the optimisation process. The binary object mapped onto the surface of the sphere is that shown in Figure 2.3. All the faces of that object have equal area. However, after the initial mapping to the sphere ( 0 iterations), the areas of the faces vary considerably. After 64 iterations, all the faces have equal area, and distortions are minimised (figure from Brechbühler, 1995).

2. As the mapping of the individual faces of an object surface onto a sphere produces distortions, it is necessary to optimise the distribution of points on the sphere. This process attempts to make the shape of all the mapped faces as similar to their 
original square form as possible. This is solved by non-linear optimisation (Gill et $a l ., 1981$ ) - an iterative process whereby a function (in this case, a measure of distortion) is minimised according to constraining criteria (for example, that an object surface region must map to a region of proportional area on the sphere). Figure 2.4 shows the distribution of points on the sphere following various iterations of the optimisation process. In the final figure (labelled 64), all faces have equal area, and distortions are minimised.

3. Finally, the representation of the object on the surface of a sphere, allows for the expansion of the surface into a series of spherical harmonic functions. As an example, the shape illustrated in Figure 2.2 may be described using the following equations (these are spherical harmonics up to degree 1, and would therefore describe the smooth surface illustrated in Figure $2.2 \mathrm{~b}$ - increasing the number of harmonics, would increase the number of terms in the equations ${ }^{1}$ ):

$$
\begin{aligned}
& x(\theta, \phi)=4.032 \frac{1}{\sqrt{\pi}}-0.405 \sqrt{\frac{3}{\pi}} \operatorname{Cos} \theta+4.072 \sqrt{\frac{3}{8 \pi}} \operatorname{Cos} \phi \operatorname{Sin} \theta-2.745 \sqrt{\frac{3}{8 \pi}} \operatorname{Sin} \phi \operatorname{Sin} \theta \\
& y(\theta, \phi)=4.830 \frac{1}{\sqrt{\pi}}+0.405 \sqrt{\frac{3}{\pi}} \operatorname{Cos} \theta-4.072 \sqrt{\frac{3}{8 \pi}} \operatorname{Cos} \phi \operatorname{Sin} \theta-2.745 \sqrt{\frac{3}{8 \pi}} \operatorname{Sin} \phi \operatorname{Sin} \theta \\
& z(\theta, \phi)=5.378 \frac{1}{\sqrt{\pi}}-0.971 \sqrt{\frac{3}{\pi}} \operatorname{Cos} \theta+3.397 \sqrt{\frac{3}{8 \pi}} \operatorname{Cos} \phi \operatorname{Sin} \theta-4.066 \times 10^{-7} \sqrt{\frac{3}{8 \pi}} \operatorname{Sin} \phi \operatorname{Sin} \theta
\end{aligned}
$$

These equations may be simplified, so that they are expressed in terms of the spherical harmonic base equation, and the coefficients. In matrix form, they appear as shown below - multiplying out the matrices gives the equations above :

\footnotetext{
${ }^{1}$ Spherical harmonics to degree 0 contribute one term to each equation, and the spherical harmonic of degree 1 contribute three additional terms to each equation. As the number (or degree) of spherical harmonics increase, two additional terms are introduced, thus spherical harmonics to degree 2 contribute five terms, giving a total of $1+3+5=9$ terms in each equation.
} 


$$
X(\theta, \phi)=\left[\begin{array}{lll}
x(\theta, \phi) & y(\theta, \phi) & z(\theta, \phi)
\end{array}\right]=\left[\begin{array}{c}
1 / \sqrt{\pi} \\
\sqrt{3 / \pi} \operatorname{Cos} \theta \\
-\sqrt{3 / 8 \pi} \operatorname{Cos} \phi \operatorname{Sin} \theta \\
-\sqrt{3 / 8 \pi} \operatorname{Sin} \phi \operatorname{Sin} \theta
\end{array}\right]\left[\begin{array}{ccc}
4.032 & 4.830 & 5.378 \\
-0.405 & 0.405 & -0.971 \\
-4.072 & 4.072 & -3.397 \\
2.745 & 2.745 & 4.07 \times 10^{-7}
\end{array}\right]
$$

Again, when the parameters $\theta$ and $\phi$ lie in the range stated above, $X(\theta, \phi)$ defines points on the surface of the object, thus providing a mathematical description.

\subsubsection{Modelling the shape of the ferret LGN.}

\subsubsection{Data collection.}

The location of the LGN was established in each animal, and the sections containing the most dorsal and ventral points were determined. The outline of the LGN was traced from all sections between the two points noted using a camera lucida (magnification $\times 500$ ). Anatomical details such as the borders of the thalamus, the border of the third ventricle, hippocampus and dentate gyrus were also included.

Prior to stacking each section on top of each other for three-dimensional reconstruction, alignment of each section relative to each other was necessary. Alignment was always started from a section taken to be approximately mid-way between the dorsal and ventral poles of the nucleus. Three points forming an isosceles triangle were marked (with a pin) on the first tracing. The next section was then placed below this central section, and the tracings rotated and translated by hand. The sections were considered to be aligned when all features drawn (LGN and the additional detail as described above) were suitably superimposed as determined by eye. A pin was then passed through the marks in the top section in order to create a new set of marks on the lower section, and the process repeated until all sections were aligned. 
The outline of each LGN was digitised with a graphics tablet. Each outline was placed on the tablet, and aligned with three points corresponding to the points marked on each tracing. The first section digitised was always the most ventral. The final result was a data file containing the $x, y$ co-ordinates of each outline, each separated by an indicator flag.

\subsubsection{3D reconstruction and processing.}

Given the co-ordinates of each outline, the creation of a three-dimensional binary image proceeded as follows :

1. The minimum and maximum co-ordinates in the $x, y, z$ directions were established in order to calculate the size of the three-dimensional volume.

2. All voxels in the volume were set to black (i.e. the voxels were 'off', value $=0$ ), except for those where a co-ordinate was defined, in which case were set to white (i.e. the voxels were 'on', value $=1$ ).

3. A flood-filling algorithm was employed to set each voxel to white if they were defined as being inside the interior of the outline.

Software was written to perform all these tasks $(\mathrm{ClC}++)$, the results of the process being a binary three-dimensional image of the LGN (in reality, this was a binary file, which when interpreted according to specific rules, would represent a 3D image). This is illustrated in Figure 2.5, in both two and three dimensions.

As can be seen from Figure 2.5b (see also Figure 2.6a), the reconstruction appears ragged. A variety of factors contribute to this :

- The borders of the LGN were not always clearly defined in histological sections.

This could be due to the anatomy itself, or the histological procedure. 
- The alignment procedure may not have been very accurate, although this could be a

bi-product of the first factor.

- The digitising stage could also introduce a degree of error.

$\underline{A}$

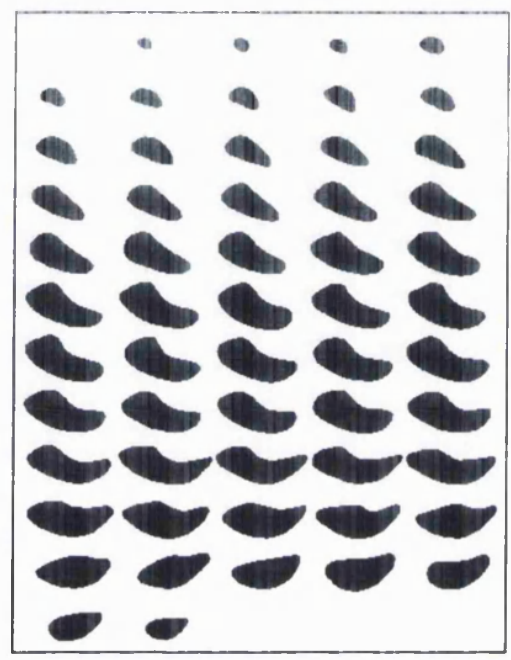

$\underline{B}$

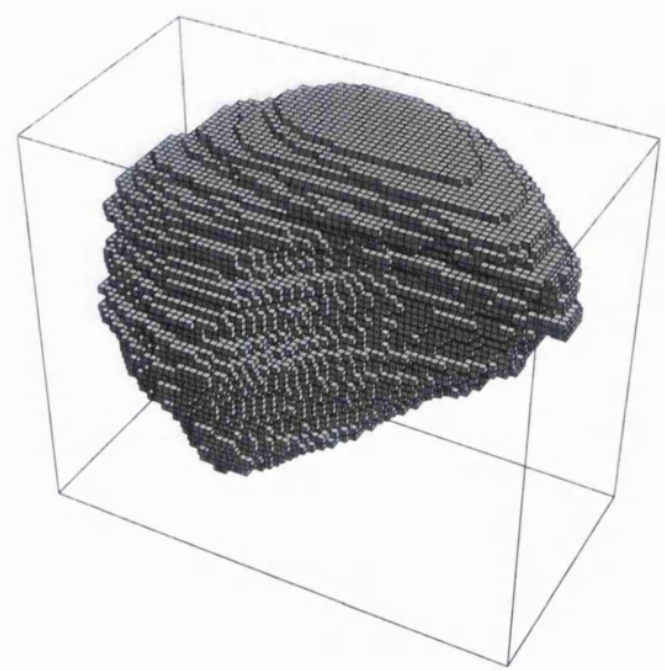

Figure 2.5 - Illustration of the creation of a binary 3D volume. A shows each individual section as traced from the microscope, arranged in the order ventral to dorsal, left to right. $\underline{B}$ illustrates the representation of the same data as a three-dimensional binary volume, where each individual section has been stacked on top of the next. Every pixel in $\underline{A}$ represents a voxel in $\underline{B}$, where the depth of each voxel (i.e. the $\mathrm{z}$-dimension) is determined by the thickness of the histological section. The data is from a P10 animal.

To correct for the apparent raggedness, a three-dimensional convolution filter was applied. The application of such filters to images is primarily for noise removal, and is achieved by averaging the image over a specified region (Gonzalez and Woods, 1992). The filter used here was analogous to the standard two-dimensional convolution filters in conventional image-processing, but here, extended to three dimensions. The filter was applied initially to the original data, and thereafter, repeatedly to the result of previously filtered images until the desired degree of noise reduction was achieved as determined by eye. On average, 3 iterations of filtering were applied. Thereafter, the 
final filtered image was thresholded back to a binary data volume. The final result of

filtering is illustrated in Figure 2.6.
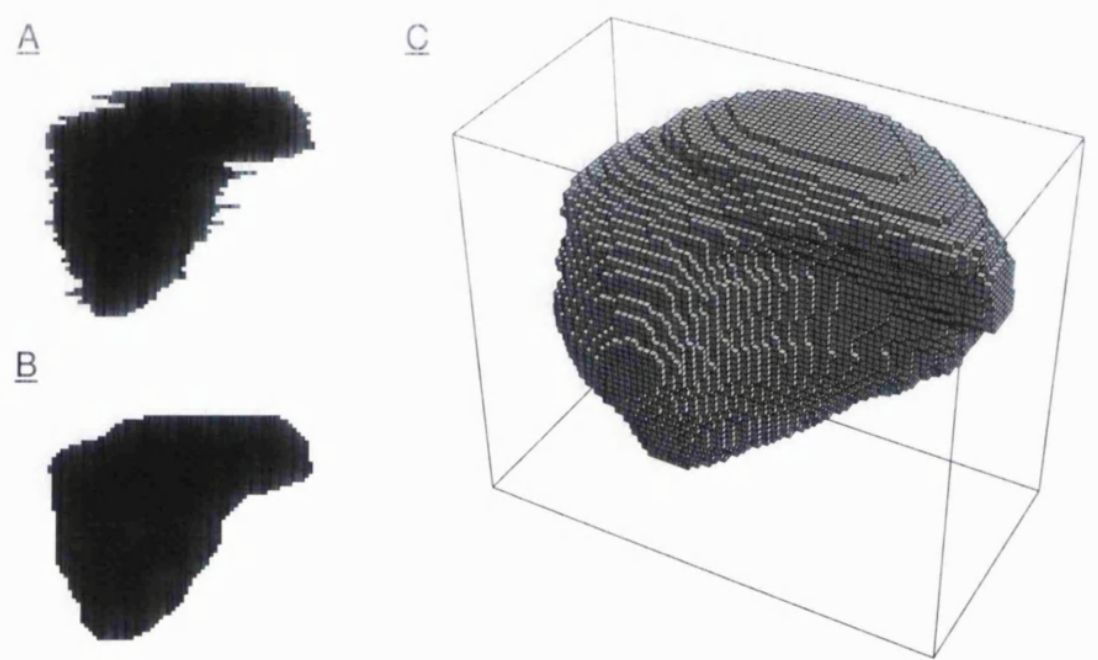

Figure 2.6 - Results of applying the 3D convolution filter. A shows a cross-section of the original volume, illustrating the imprecision of the reconstruction process. This is analogous to viewing the volume in a sagittal plane, in this case, the most ventral section is towards the bottom of the image. $\underline{B}$ shows the same cross-section after 3 iterations of the filter, while $\underline{C}$ shows the full 3D volume (compare this with Figure 2.4b).

\subsubsection{Calculation of shape coefficients.}

Section 2.5.1. described the steps in the model building process. The first two stages are referred to as the parameterization stage, and involve the majority of work (especially in terms of computer time). The software to perform the parameterization was provided by Dr. Brechbühler (Swiss Federal Institute of Technology, Zurich). This was custom written for their particular computer systems, and took advantage of numerous mathematical and graphical libraries at their disposal. The source code was therefore heavily modified and adapted to make use of the facilities available at the Institute of Ophthalmology: a PC running the Linux operating system. All code was written in a combination of $\mathrm{C}, \mathrm{C}++$ or FORTRAN, and compiled using the freely available GNU C and FORTRAN compilers. 
The output of the parameterization program was used for the calculation of spherical harmonic functions and their coefficients. This was achieved using a program written to run under the Mathematica system (Wolfram, 1991). All models calculated in this study employed spherical harmonic functions to degree 8 .

\subsubsection{Normalisation of coefficients.}

To make meaningful comparisons between shapes, it is necessary to normalise the coefficients describing them. The method described here allows for shape normalisation by transformations of the shape coefficients themselves.

The first type of normalisation, and one which was applied to all the models created here, is that for the position of the model as fitted to the original data (parameter space normalisation). Figure 2.7a shows the resulting model fitted to the data shown in Figure 2.6c with no normalisations applied. The two poles (i.e. the points at which the longitude lines converge) are situated on the points at which the dataset begin and end (arrows, Figure 2.7a). To remove this dependency on the dataset, the model is rotated about the data, such that the poles are at either ends of the shortest axis of the first degree harmonic (arrow, Figure $2.7 \mathrm{~b} \&$ Figure $2.7 \mathrm{c}$ ). This normalisation was deemed necessary since each dataset did not have the same starting point.

The remaining normalisations apply to the position, rotation, and size of the object in space (object space normalisations). Position (or translation) normalisation is achieved by adjusting the first coefficient so that its components are equal to zero. This translates the centre of the first harmonic ellipsoid to the origin of a standardised co-ordinate space (Figure 2.8a) and was applied to all models. This was due to the 
fact that the models did not incorporate any information concerning the precise location of the LGN within the brain. Rotation normalisation may be applied, by rotating the first harmonic so that its main axes are aligned with the co-ordinate axis of a standardised co-ordinate space (Figure 2.8b). Finally, normalisation for size is accomplished by scaling the first harmonic, and adjusting every other coefficient accordingly. This implies that the shape being modelled can be made to have any size whatsoever. Rotation and size normalisations were applied according to the type of analysis being carried out, and are referred to in the sections which follow.
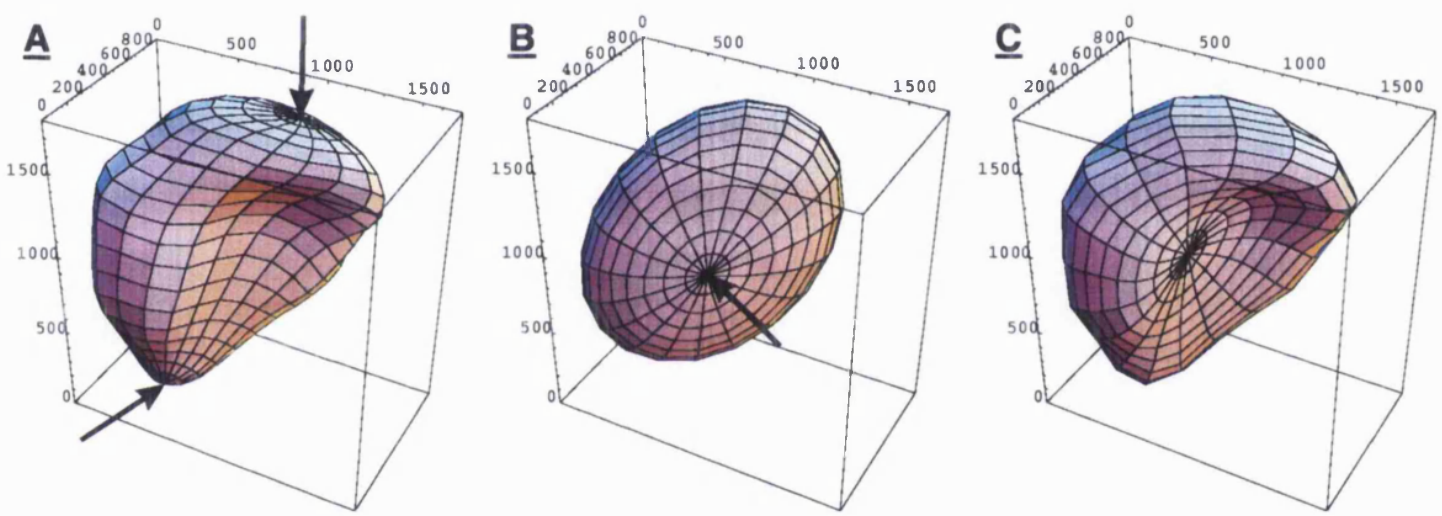

Figure 2.7 - An illustration of the concept of parameter space normalisation. $\underline{\text { A shows the }}$ original fit of the model to the data shown in Figure 2.5c. The dependency on the beginning and end points of the data set are removed by rotating the model about the data such that the poles of the model are at either ends of the shortest axis of the $1^{\text {st }}$ degree harmonic as shown in $\underline{B}$. The resulting model with harmonics to degree 8 are shown in $\underline{\mathrm{C}}$. 

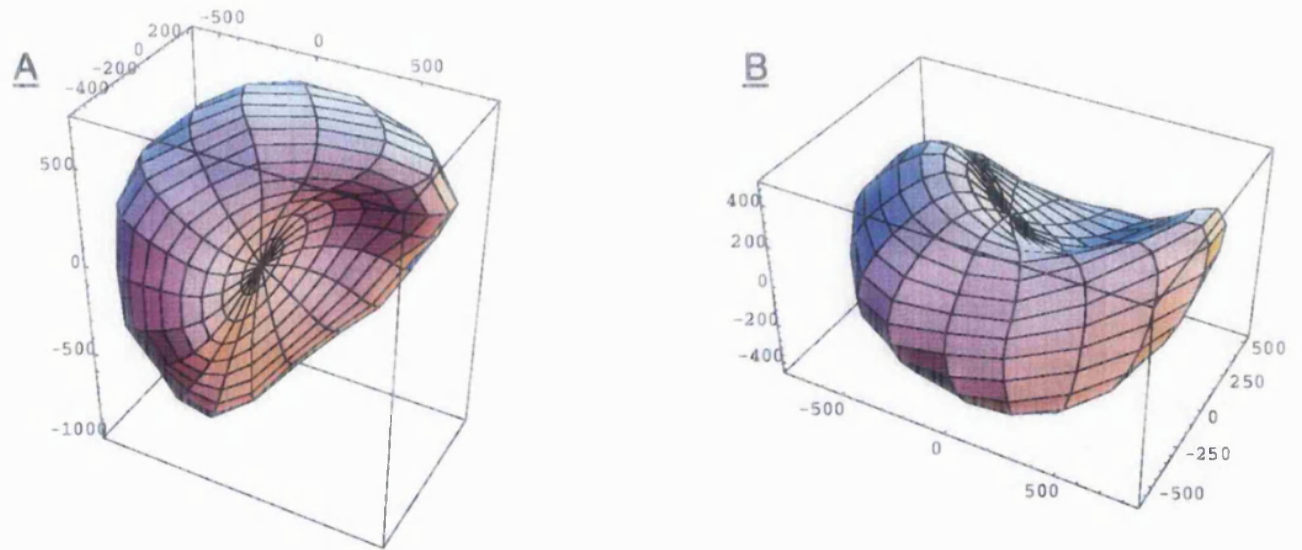

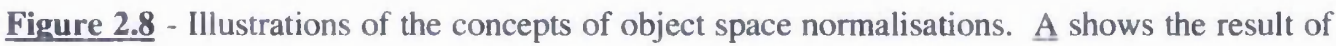
position normalisation, where the centre of the $1^{\text {st }}$ harmonic ellipse is translated to the origin of a standardised co-ordinate space. $\underline{B}$ shows the result of rotation normalisation, where the $1^{\text {st }}$ harmonic ellipse is rotated such that its major (longest) axis is aligned with the first axis of the standardised co-ordinate space. Normalisation for size is not shown - it simply results in a scaling of the standardised co-ordinate space axes.

\subsection{Analysis of growth.}

Some data presented here describe growth processes, and for this reason are fitted with logistic (or growth) curves (Antonelli, 1985; Eggermont, 1985). The logistic function produces an s-shaped curve with a flat upper plateau, and is very characteristic of a number of biological processes. It is an extension of Huxley's (Huxley, 1932) original concept of exponential organism growth and is defined by:

$$
y(t)=\frac{\alpha}{1+e^{\beta-x}}
$$

where $\alpha, \beta$, and $\gamma$ are three parameters determined in order to minimise the sum of squared errors between the original data and the curve. $t$ is the post conceptional age in days, and $e$ the exponential constant $(=2.718)$. The parameters $\alpha, \beta$, and $\gamma$ were determined using the Levenberg-Marquardt algorithm (SPSS Inc., 1993) where $\alpha$ determines the value at which the curve plateaus, $\beta$ determines the point of inflexion, or the time of maximum growth, and $\gamma$ determines the rate of growth (Figure 2.9). 

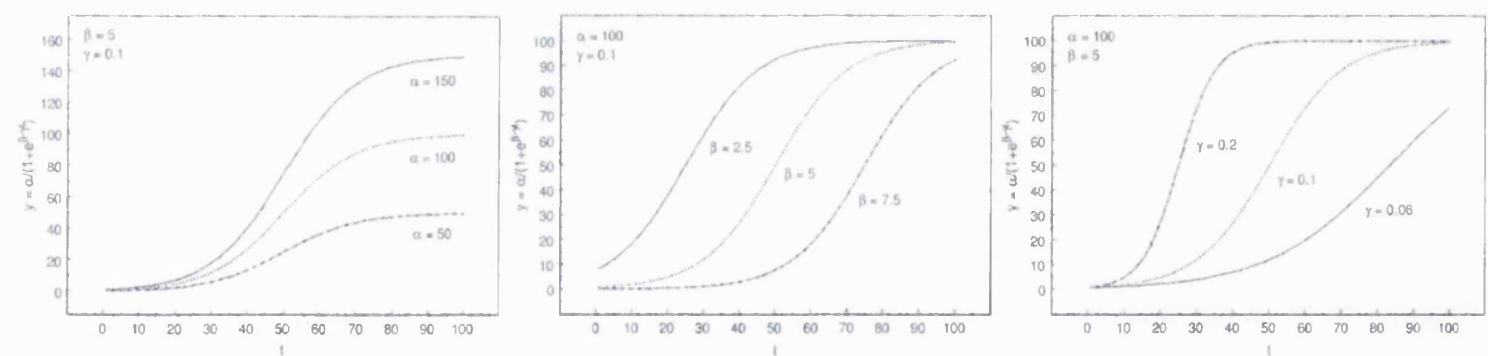

Figure 2.9 - Illustration of how the parameters $\alpha, \beta$ and $\gamma$ determine the shape of the logistic curve (eq. 2.1). $\alpha$ determines the value at which the curve plateaus, $\beta$ determines the point of inflexion, or the time of maximum growth, and $\gamma$ determines the rate of growth.

For comparative purposes, the logistic curve can be normalised by setting the value $\alpha=100$. This expresses the process of growth in terms of a percentage, while differentiating the function provides information on the rate of growth (eq. 2.2).

$$
\frac{d y}{d t}=\frac{\alpha e^{\beta-\gamma} \gamma}{\left(1+e^{\beta-\gamma}\right)^{2}}
$$

In order to explore the relationships between different features fitted with the above curve, equation 2.1 can be rearranged to eliminate $t$. For example, if LGN volume and cell size were defined by the equations:

$$
y(t)=\frac{\alpha}{1+e^{\beta-\gamma}}, \text { and } z(t)=\frac{\delta}{1+e^{\kappa-\lambda}}
$$

then LGN volume can be expressed as a function of cell size by:

$$
y(z)=\frac{\alpha}{1+e^{\beta}\left[\frac{z e^{\kappa}}{\delta-z}\right]^{-\frac{\gamma}{\lambda}}}
$$




\section{Ipsilateral \& contralateral projections to the developing LGN.}

\subsection{Introduction.}

One of the main inputs to the LGN comes from the retina. In many mammalian species, the projections are bilateral, and result in binocular maps within the nucleus. The projections from each eye terminate in adjacent layers of the LGN, but are in conjugate register, such that there is a continuity in the representation of the visual field (Kaas et al., 1972). In the ferret, RGC axons from each eye first reach the LGN at approximately E25-E28 (Embryonic day 25-28, Cucchiaro and Guillery, 1984; Johnson and Casagrande, 1993). Between E32 and P0 (the day of birth), projections from both eyes are mixed throughout the nucleus (Cucchiaro and Guillery, 1984), but each has been shown to be arranged in the form of an orderly retinotopic map (Jeffery, $1985 ; 1989 ; 1990)$.

During the first postnatal week, studies have shown that the maps must shift in relation to one another to form the pattern of binocular segregated registration found in the adult ferret (Jeffery, 1989; 1990). In the rat, this process of registration between projections from each eye arises as a consequence of the increase in the size of the 
LGN, rather than changes in the size of the two developing projections (Jeffery, 1984).

This study showed that the proportion of LGN occupied by the ipsilateral projection declines during development (Figure 3.1a), but is mainly a consequence of the relative growth of the nucleus, since the volume of the terminal region remains fairly constant (Figure 3.1b). Additionally, after retraction is complete LGN volume increases, along with a proportional increase in terminal field volume, such that the percentage of LGN occupied by the ipsilateral field remains the same following retraction.
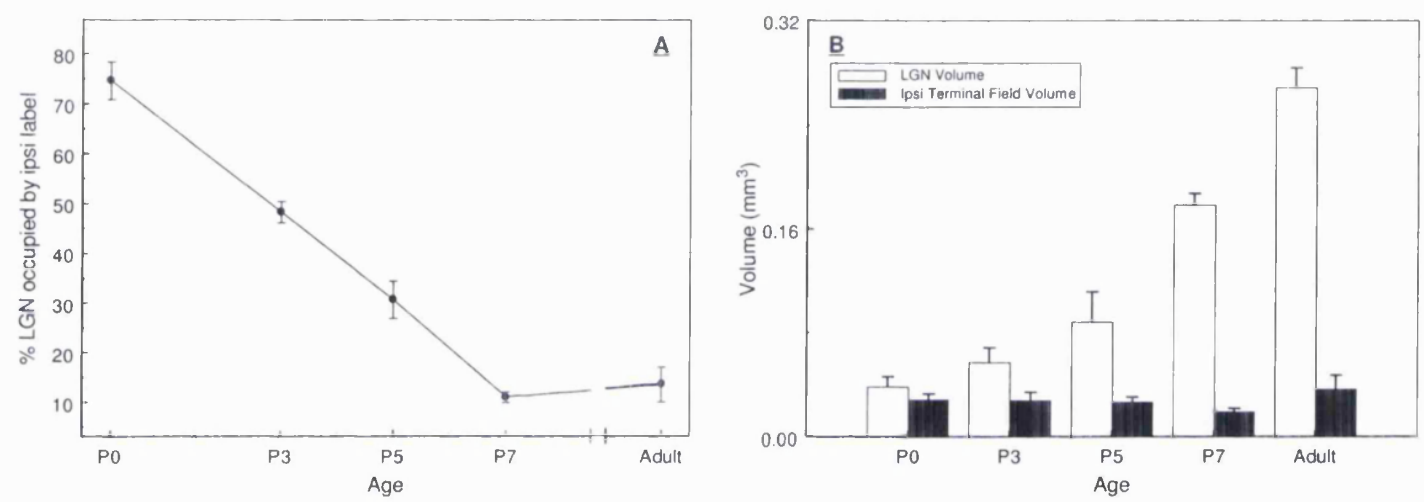

Figure 3.1 - (A) Proportion of the rat LGN at successive developmental stages occupied by RGC terminal fields following an injection of HRP into the ipsilateral eye. (B) Actual volumes of the LGN and the terminal field of the ipsilateral eye. (From Jeffery, 1984)

Changes in the structural configuration of the LGN must therefore play some role in the development of registration between binocular retinotopic maps. Without a description of these changes in LGN shape, it will not be possible to determine their relationship with the process of registration. These issues are examined in the chapters which follow, but here, ocular segregation is investigated in the ferret in order to determine whether a similar pattern of LGN and terminal field growth as seen in the rat can be observed. While the developmental process of ocular segregation in this animal has been previously demonstrated (Linden et al., 1981; Cucchiaro and Guillery, 1984), no quantitative measurements have been made on the magnitude of the terminal fields. 


\subsection{Methods.}

Tissue and sectioned material were prepared according to the protocol described in Section 2.3. The outline of the LGN and the patterns of terminal label on either side of the brain were drawn with the aid of a drawing tube. The diagrams were digitised with a graphics tablet, and respective volumes calculated.

\subsection{Results.}

Injections of HRP were successfully delivered into a single eye of 14 ferrets (2 P0, 3 P5, 3 P8, 4 P12, and 2 adults; Figure 3.2). The pattern of labelling seen in the nucleus is characteristic of that previously described in this animal (Linden et al., 1981; Cucchiaro and Guillery, 1984). On the day of birth, label is present throughout the nucleus on both sides of the brain, indicating that retinal afferents from both eyes innervate all regions of the nucleus (see Figures $12 \& 13$ in Linden et al., 1981). By P5, the uncrossed afferents are restricted to a small region at the caudal pole of the nucleus, and simultaneously, the crossed afferents retreat from a similar region (asterisk, Figure 3.2). At this stage, there is a degree of overlap between the two sets of afferents.

At the beginning of the second postnatal week (P8, not shown here), the region occupied by ipsilaterally projecting afferents becomes more well defined, as does the region vacated by the contralateral afferents. By $\mathrm{P} 12$, these regions are virtually adult like, and crossed and uncrossed afferents have almost completed retraction into their appropriate regions (Figure 3.2). There is still a small degree of overlap between the projections, but segregation is complete shortly after this period (Linden et al., 1981). 
Figure 3.2 also shows that a certain degree of shape change is apparent in the borders defining the terminal field regions.
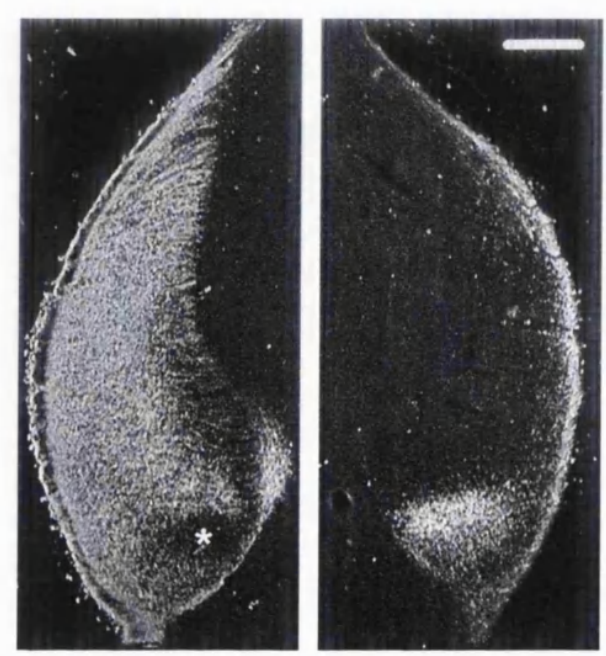

\author{
P5 \\ Scale Bar: $250 \mu \mathrm{m}$
}
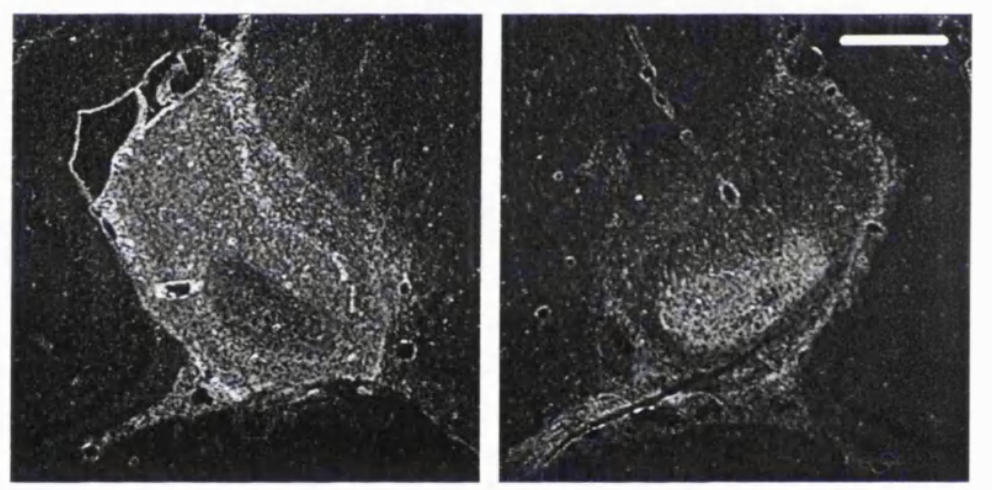

P12

Scale Bar: $250 \mu \mathrm{m}$
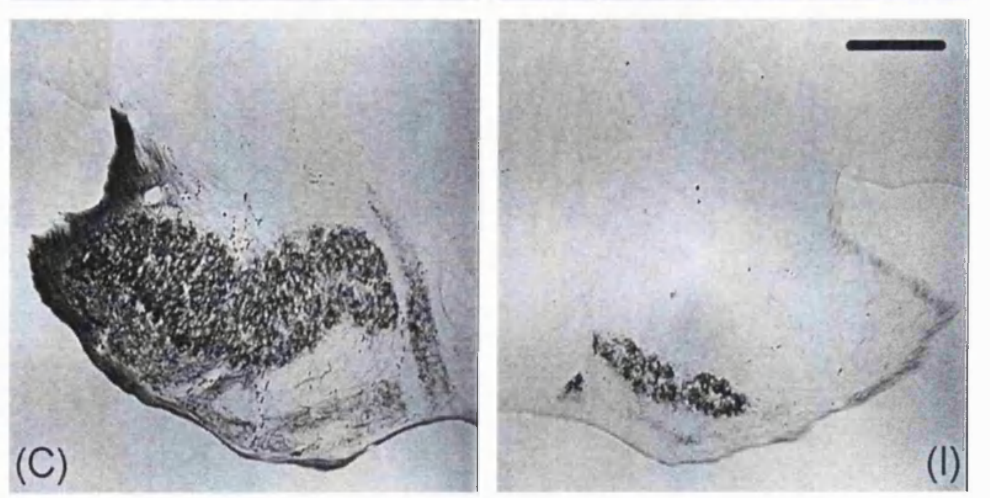

Adult

Scale Bar: $500 \mu \mathrm{m}$

Figure 3.2 - Horizontal sections through the LGN of the ferret ipsilateral (I) and contralateral (C) to an intraocular injection of HRP at the ages indicated. The sections are taken from midway between the dorsal and ventral poles of the nucleus, and rostral is towards the top. To further highlight the regions occupied by the crossed and uncrossed projections, sections from P5 and P12 animals are shown in darkfield. The dark appearance of the labelled regions in the P12 animal is due to an additional Nissl counterstain. 
Figure 3.3 shows the expanding volume of the LGN during development, and also shows the volume of space occupied by ipsilaterally projecting RGC terminals (also summarised in Table 3.1). Following birth, the volume of the LGN increases steadily during the first two weeks, and by adulthood, has increased by more than four-fold. In contrast, the volume of the ipsilateral terminal field appears to fall during early development, prior to an increase in adulthood. However, statistical tests reveal no significant differences between the means of the terminal field volumes between P0 and P12 (Kruskal-Wallis 1-way ANOVA, $p=0.082$ ), allthough this could be due to the low number of animals within each group. The opposite is true for the volume of the LGN between the same age range $(p=0.0312)$. Thus it appears that the volume of the LGN increases during the process of retraction, while the volume of the ipsilateral terminal field remains the same.

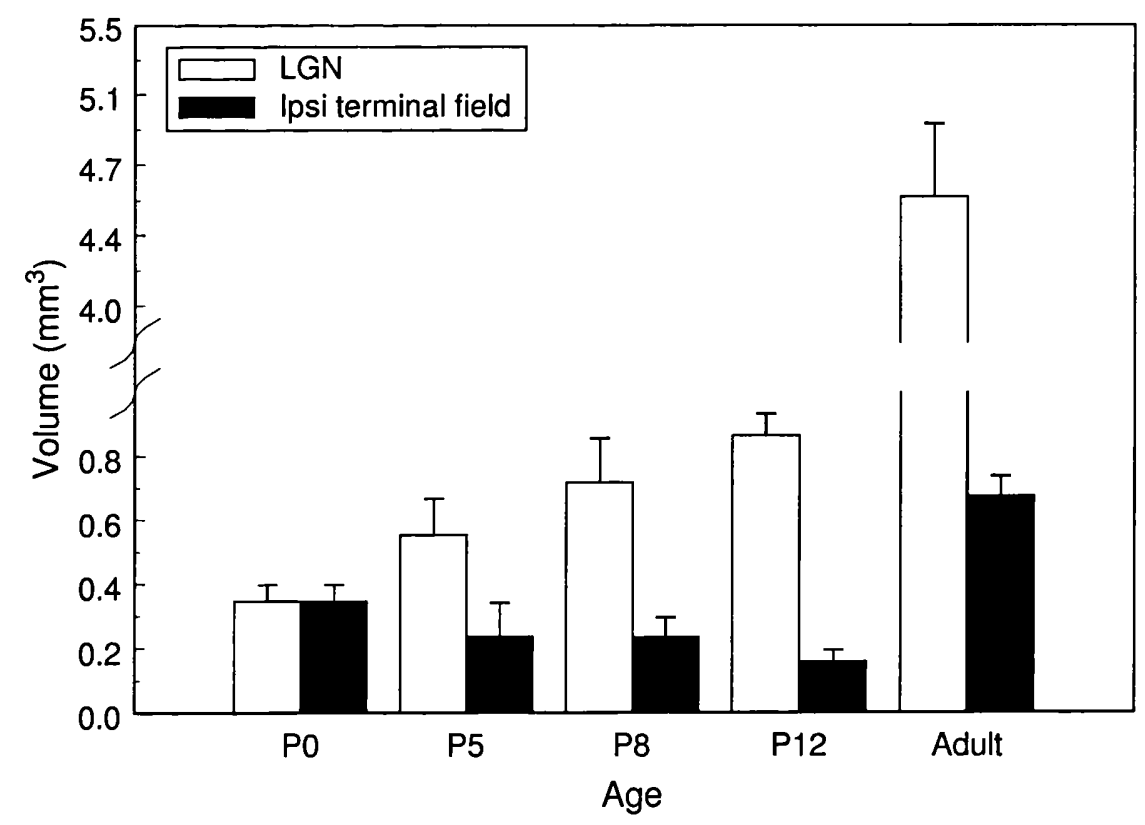

Figure 3.3 - Volume of the LGN $\left(\mathrm{mm}^{3}\right)$ as measured in frozen sectioned material, together with the volume of the region occupied by ipsilaterally projecting ganglion cell terminals. Note that the ordinal scale is linear, but is interrupted to illustrate the detail of the younger animals. 
The proportion of the entire LGN occupied by terminals from the ipsilateral eye, and the substantial decline which occurs between early infancy and adulthood is shown in Figure 3.4. The relative proportions stabilise at around P12, indicating that between this point and adulthood, both the LGN and ipsilateral terminal field grow proportionately to each other.

\begin{tabular}{cccc}
\hline Age & Nucleus Volume & Ipsilateral Label & Contralateral Gap \\
\hline P0 & 0.3058 & - & - \\
& 0.3909 & - & - \\
P5 & 0.4423 & 0.1175 & 0.0708 \\
& 0.5353 & 0.2783 & 0.1035 \\
& 0.6799 & 0.3151 & 0.0161 \\
P8 & 0.6922 & 0.2252 & 0.0408 \\
& 0.8796 & 0.2983 & 0.0461 \\
& 0.5814 & 0.1821 & 0.0644 \\
P12 & 0.7801 & 0.1084 & 0.0878 \\
& 0.8870 & 0.1709 & 0.1241 \\
& 0.8532 & 0.1831 & 0.1702 \\
Adult & 0.9392 & 0.1744 & 0.1846 \\
& 4.3000 & 0.6272 & 0.7363 \\
\hline
\end{tabular}

Table 3.1 - Developmental changes in total volume $\left(\mathrm{mm}^{3}\right)$ and terminal field size $\left(\mathrm{mm}^{3}\right)$ in the pigmented ferret following an injection of HRP into one eye. Nucleus volume is calculated from the average of both left and right nuclei. At P0, terminal field label is evident throughout the nucleus on both sides of the brain, and therefore the volume of label is the same as the volume of the nucleus.

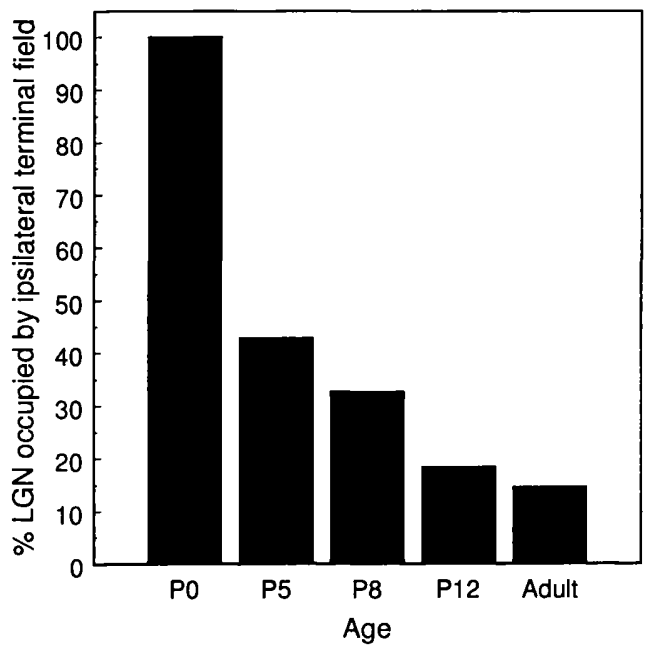

Figure 3.4 - Mean percentage of the LGN occupied by terminal label following an injection of HRP into the ipsilateral eye. The values are calculated as the ratio of the ipsilateral label to the nucleus volume. The ratios stabilise around $\mathrm{P} 12$ indicating that LGN volume and terminal field volume increase proportionately after this point. 


\subsection{Discussion.}

The results presented here confirm the previous qualitative findings of Linden et al (1981), and show that the segregation of the ocular distinct laminae within the LGN occur during the first two postnatal weeks. Measurements made on total volume of the ipsilateral terminal field show that there are no statistically significant increases in its size during the period of retraction. This is in contrast to the significant changes in the volume of the nucleus over a similar period. Furthermore, between the time at which segregation is complete (the end of the second postnatal week), and the time at which the nucleus reaches its mature adult state, the volume of the LGN and its ipsilateral terminal field increase proportionally in size - i.e. the percentage of LGN occupied by ipsilateral terminal field label does not increase after segregation is complete.

Additionally, the results are consistent with the findings of Jeffery (1984) in showing that the uncrossed terminal field remains the same size, while the LGN grows during the period of segregation. Similar results have been reported in another carnivore, the cat (Shatz, 1983). This study showed a similar change in the proportion of LGN occupied by terminals from the ipsilateral eye, but did not specify whether this was a result of the growth of the nucleus while the terminal field size remained constant.

If the volume of the ipsilateral terminal field remains the same during the period of segregation, but at the same time retracts to the posterior aspect of the nucleus, and changes in shape, it is practicable to question what role differential growth of the LGN might play in the process. This hypothesis that differential growth of the LGN might play a role in ocular segregation is novel, and has not been explored to date. In 
addition, there are no good quantitative descriptions of the changes in LGN structure to eliminate the possibility.

However, if shape change is a means by which the ipsilateral projection assumes its adult configuration, how can the same shape changes be influencing the development of the contralateral projection? Similar to the ipsilateral projection, this occupies the entire nucleus on the day of birth, but during development, vacates a region of posterior LGN, which is sufficient for the ipsilateral projection to occupy. These may be considered as two opposing events: a movement of the ipsilateral projection caudally, while the contralateral projection moves rostrally. Shape change could be envisaged as a mechanism for both these events, although if changes act on one projection only, then the retraction of the ipsilateral projection to the caudal pole of the nucleus would have to force the contralateral projection out of the same region, or vice versa.

Previous studies have led to the concept of segregation as a consequence of competitive interactions between the two eyes: if one eye is removed early in development prior to ocular segregation, the terminals fail to segregate into distinct laminae within the LGN (Thompson et al., 1993). However, it has been established that performing monocular enucleations has an adverse effect on the growth of the LGN (Guillery et al., 1985), and therefore the effect of removing one eye could be interpreted as removing one of the factors which may play a role in segregation. 


\section{A cellular analysis of the ferret $L G N$.}

\subsection{Introduction.}

The previous chapter introduced the idea that differential growth of the LGN may play a role in the development of ocular segregation. Hence, the factors regulating the changing configuration of this nucleus are of interest. These factors may be split into two distinct types: changes may occur as a result of extrinsic factors, such as mechanical constraint, or alternatively, as a result of intrinsic factors, such as alterations in the constituent cellular population of the LGN (Van Essen, 1997), although the two are not necessarily mutually exclusive.

This chapter focuses mainly on the latter issue, and investigates parameters of the developing cellular population to explore their potential role in LGN growth. Aspects of the cellular population of the remaining thalamic region are also examined to determine whether cellular changes in the LGN are distinct from those of the thalamus. Stereological techniques have been used to calculate LGN volume, cell density and total LGN cell number (Braendgaard and Gundersen, 1986; Gundersen, 1986; but see also Guillery and Herrup, 1997). The magnitude and distribution of cell sizes have also been established. Finally, in addition to quantifying cell density and size, their relative distribution across the nucleus have also been explored. 


\subsection{Methods.}

All data gathered for the analysis described here were obtained from sections prepared as described in Section 2.2.

\subsubsection{Estimating total cell numbers.}

The method of estimating total cell numbers using stereological techniques has been described in Section 2.4. Different sampling schemes were necessary for younger and older animals due to size differences. Data collection was performed using an image analysis system incorporating stereological analysis software (Kinetic Imaging Ltd., UK).

Two different schemes were employed for estimates of LGN volume $\left(V_{\text {ref }}\right)$. For animals P10 and younger, point counting was performed in horizontal sections $90 \mu \mathrm{m}$ apart, using a square grid with a point density of 1 point $/ 0.128 \mathrm{~mm}^{2}$. In older animals, point counting was performed in sections which were $240 \mu \mathrm{m}$ apart, using a grid with a point density of 1 point $/ 0.031 \mathrm{~mm}^{2}$.

\begin{tabular}{lccc}
\hline Animal Age & $\leq \mathrm{P} 10$ & $\mathrm{P} 23$ & $\geq \mathrm{P} 47$ \\
\hline Optical dissector area & $100 \mu \mathrm{m}^{2}$ & $400 \mu \mathrm{m}^{2}$ & $900 \mu \mathrm{m}^{2}$ \\
Optical dissector height & $10 \mu \mathrm{m}$ & $15 \mu \mathrm{m}$ & $20 \mu \mathrm{m}$ \\
Distance between sections & $90 \mu \mathrm{m}$ & $240 \mu \mathrm{m}$ & $300 \mu \mathrm{m}$ \\
Raster frequency & $\begin{array}{c}\text { Every } 5^{\text {th }} \\
\text { frame }\end{array}$ & $\begin{array}{c}\text { Every } 10^{\text {th }} \\
\text { frame }\end{array}$ & $\begin{array}{c}\text { Every } 12^{\text {th }} \\
\text { frame }\end{array}$ \\
\hline
\end{tabular}

Table 4.1 - Sampling scheme parameters for estimating cell density in the LGN. Each section was scanned in a raster fashion, where each raster frame measured $0.01 \mathrm{~mm}^{2}$. The raster frequency entry in the table refers to the frequency at which counts were made in the raster grid.

The numerical density of cells $\left(N_{v}\right)$ within the LGN at different ages was estimated with three different sampling schemes. For clarity, these are listed in Table 4.1 above. 
Cell number $(N)$ was calculated as the product of cell density and LGN volume.

\subsubsection{Spatlal distributions of cellular densities.}

Cell densities were estimated in a single section taken from the mid-point between the dorsal and ventral poles of the LGN. Counts were made at a magnification of $\times 1000$ with the aid of an eye-piece graticule grid measuring $100 \mu \mathrm{m}^{2}$. The grid was moved in a systematic raster fashion, moving caudal to rostral, and lateral to medial, such that the entire LGN and thalamic region were sampled (Figure 4.1a). Counts were made in every location for animals younger than P23. In older animals, counts were made at every location if the grid was situated within the LGN, and in every other location (i.e. 1:2) if situated within the remaining thalamus. Cells were counted if they had a clear nucleus, but no attempt was made to distinguish between neurones or glia.

\subsubsection{Cell areas.}

Cell areas in both the LGN and thalamus were measured in the same section as used above. In the case of the LGN, images were taken at 10 uniformly distributed locations within the nucleus as shown in Figure 4.1b. Similarly, in the rest of the thalamus, images were taken from 9 uniformly distributed locations as shown in Figure 4.1b. Images of animals younger than P23 were taken at a magnification of $\times 125$, and those aged P23 and older at $\times 75$. From each location 10 cells were selected for area measurements, which were made with the aid of an image analysis system. The criteria for selecting a particular cell was that it had a clear nucleus and well defined cytoplasmic structure. For the purpose of continuity between different aspects of the cellular study, no distinction was made between neurones and glia. 


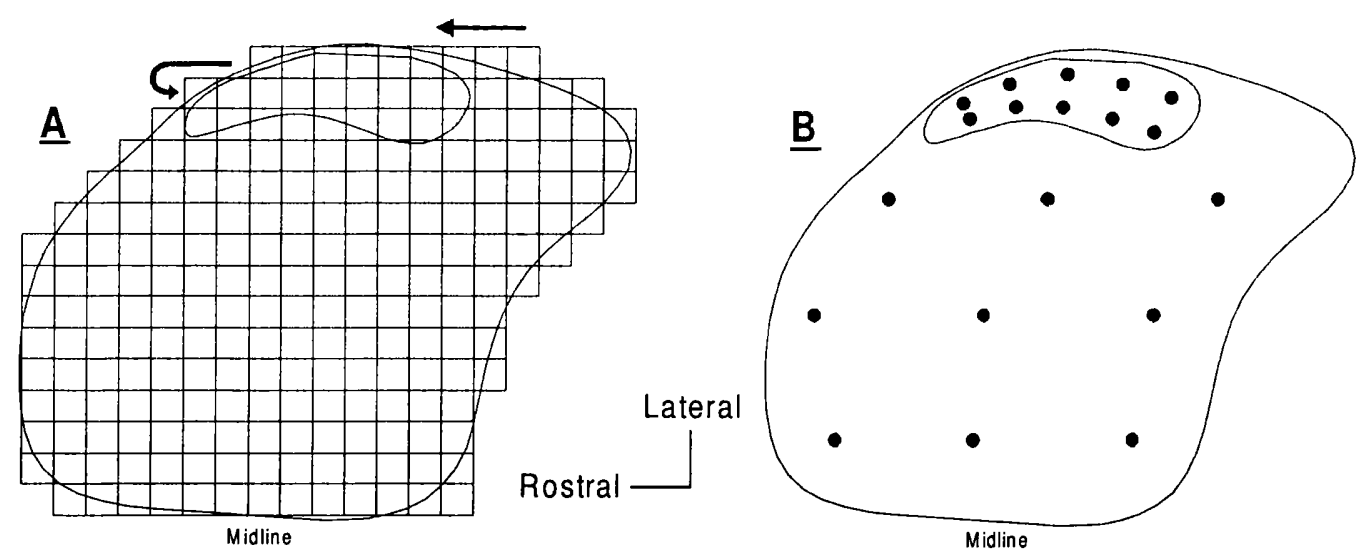

Figure 4.1 - Diagram illustrating the sampling locations for cell density (A), and cell area measurements (B) in both LGN and thalamus. The sampling grid shown in $\underline{A}$ is not to scale, but illustrates the raster movement of the counting procedure. Inevitably, the shape of both the LGN and thalamus change with the age of the animal. However, the spatial proportions of the sampling locations for cell area measurements $(\underline{B})$ were preserved. The example given above is from a $\mathrm{P} 0$ animal.

\subsection{Results.}

\subsubsection{General appearance of the LGN.}

Horizontal sections used in this study are shown in Figure 4.2 for developmental ages E38, P0, P5, P10, P23, and P83. Collectively, they illustrate some of the events occurring during the development of the nucleus, including its change in shape and rotation. The formation of cytologically distinct laminae is also evident: the cell free interlaminar zone between layers $\mathrm{A}$ and $\mathrm{A} 1$ can be distinguished in the P10, P23, and P83 animals (arrows, Figure 4.2).

The general structure and development of the nucleus is similar to that described by Linden et al (1981). The characteristic comma shape is evident at the time of birth, and transforms gradually to the L-shaped structure of the adult. Rotation is evident as a lateral movement of the monocular region such that the long axis of the nucleus moves from the rostro-caudal plane into the medio-lateral plane. 

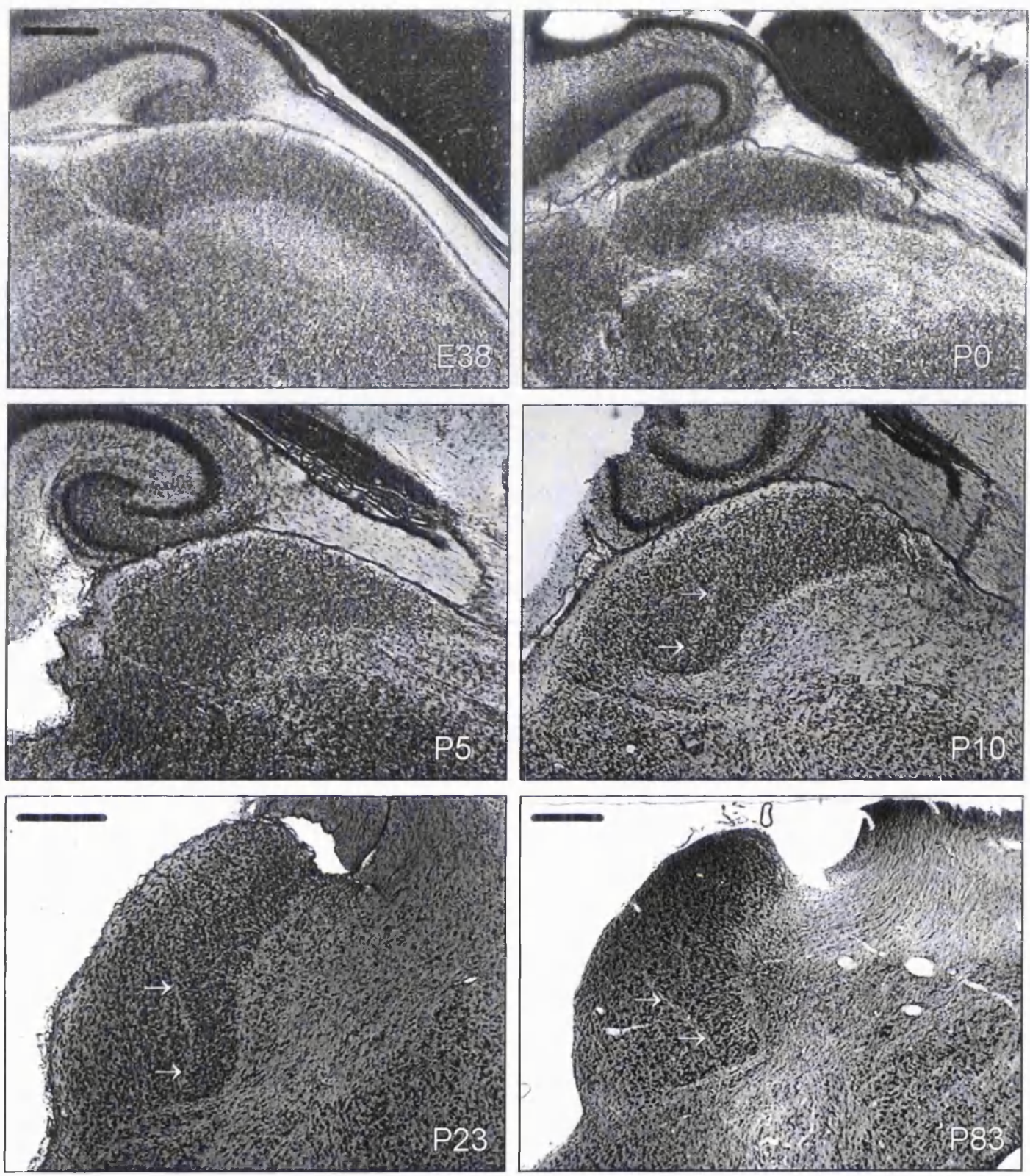

Figure 4.2 - Horizontal sections mid-way between the dorsal and ventral poles of the ferret LGN for developmental ages E38, P0, P5, P10, P23, and P83. Rostral is to the right, lateral is up. The images illustrate the key structural events occurring during development: shape change, rotation, and laminar formation. Scale bar: $250 \mu \mathrm{m}, \mathrm{E} 38-\mathrm{P} 10 ; 500 \mu \mathrm{m}, \mathrm{P} 23 \&$ P83. Arrows indicate the position of the cell free interlaminar zone separating laminae A and A1.

\subsubsection{Cellular constituents of the LGN.}

\subsubsection{LGN volume. cell densities, and cell numbers.}

Figures 4.3, 4.4 and 4.5 respectively show LGN volume, cell density, and total cell number in the entire nucleus. The expansion in LGN volume asymptotes at the 
adult value of around $3.5 \mathrm{~mm}^{3}$ approximately 40 days postnatally (Figure $4.3 \mathrm{a}$ ). The rate at which LGN volume is increasing reaches a maximum at P20-P22, expanding at a rate of approximately $0.13 \mathrm{~mm}^{3}$ per day (Figure $4.3 \mathrm{~b}$ ).

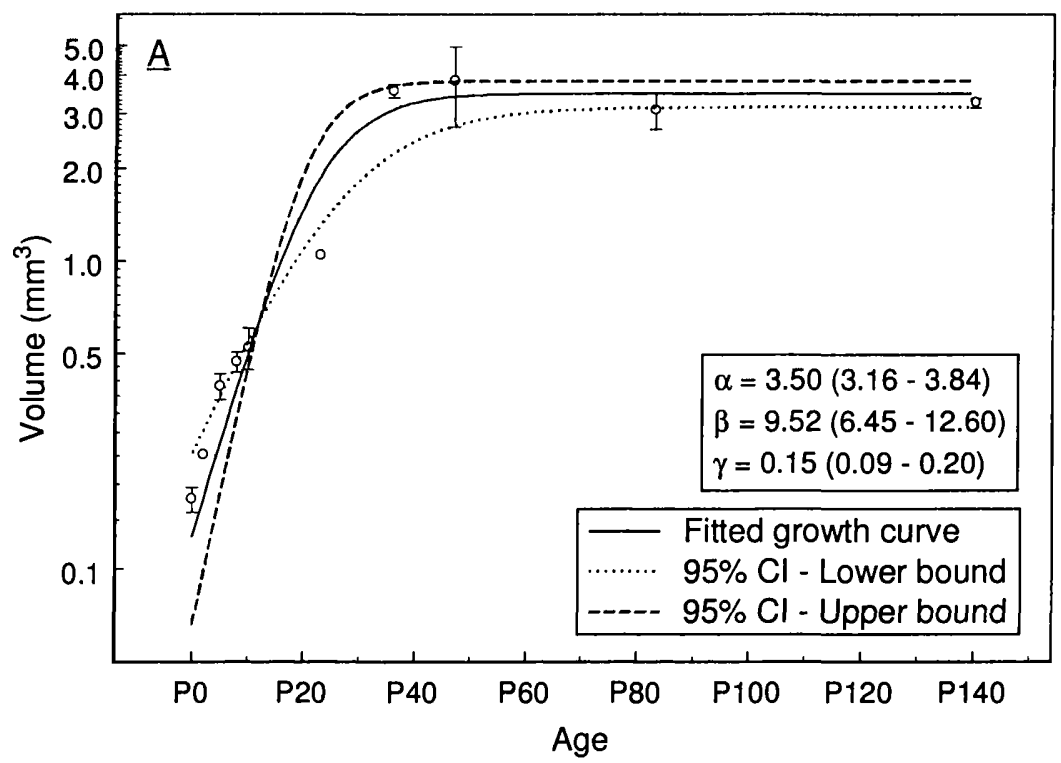

Figure 4.3 - (A) Mean volume of the LGN as estimated from the stereological methods. The $y$-axis is plotted as a logarithmic scale, and the data is fitted with a growth curve. The estimated curve parameters are shown together with $95 \%$ confidence intervals. (B) The first derivative of the growth curve provides data describing the rate of

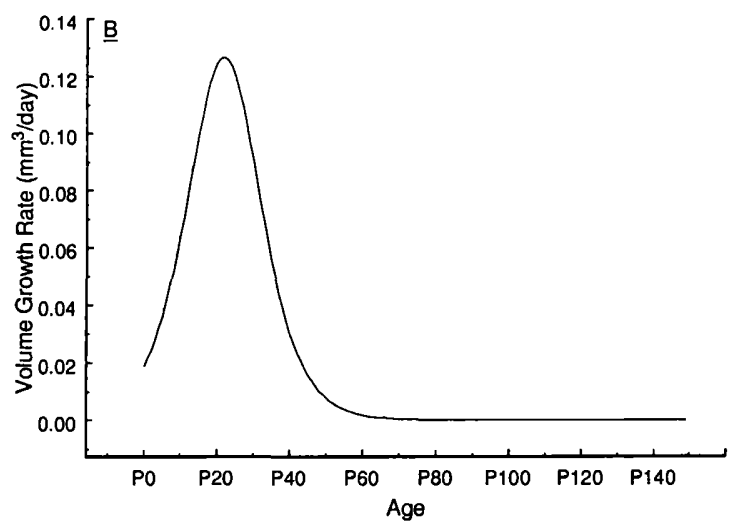
volumetric expansion.

Cell density appears to be inversely proportional to LGN volume (Figure 4.4). A peak in the density of cells is seen just after birth $\left(\approx 1,600,000 \mathrm{~mm}^{-3}\right)$, and falls over the following six week period, when the adult value is achieved $\left(\approx 100,000 \mathrm{~mm}^{-3}\right)$. Cell numbers are calculated as the product of LGN volume and cell density. Due to the multiplicative nature of this derivation, cell numbers have large standard deviations, and this is more so the case for the P47 animals (Figure 4.5). Nevertheless, a logistic growth curve has been fitted to the data with a good degree of accuracy $\left(r^{2}=0.94\right)$. At 
birth, cell numbers are around 250,000 and proliferation appears to continue until the latter part of the first postnatal week when cell numbers have more than doubled to approximately 520,000. Over the following two weeks, cell numbers decline until they reach a stable population of around 350,000 .

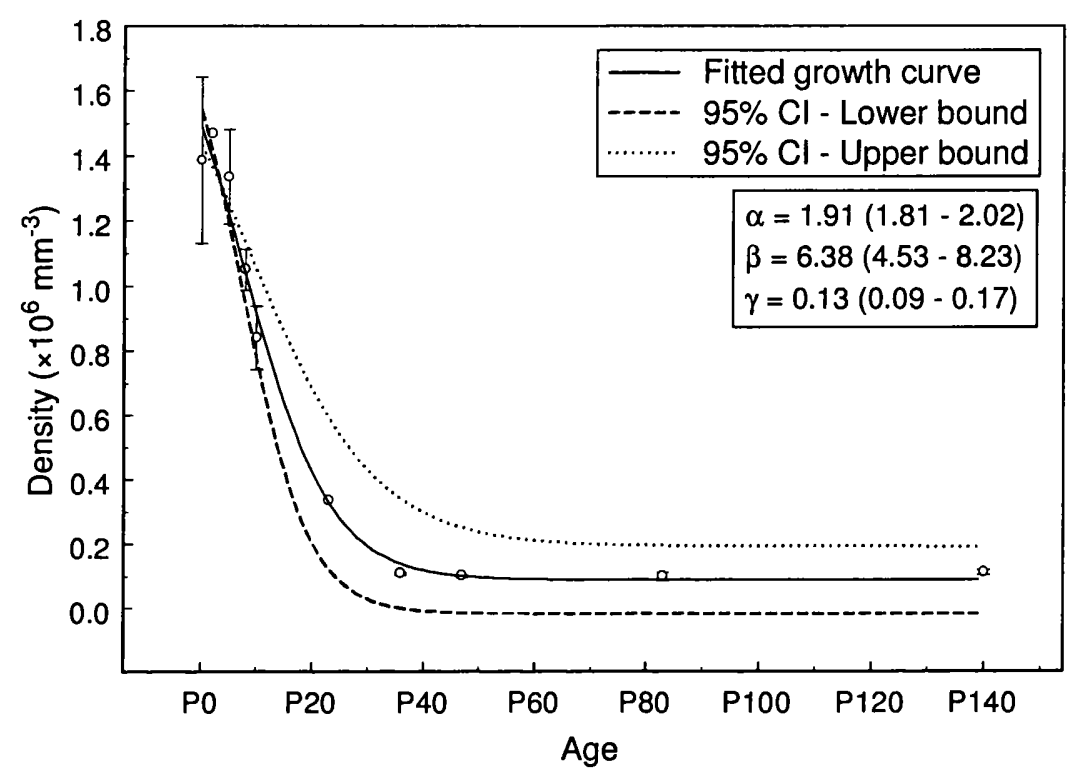

Figure 4.4 - Mean density of LGN cells as estimated using the optical dissector. The peak in cell density is seen around birth, and the adult value of approximately $100,000 \mathrm{cells} / \mathrm{mm}^{3}$ is seen after P50. The data is fitted with a growth curve, and the estimated parameters of this curve are shown together with $95 \%$ confidence limits.

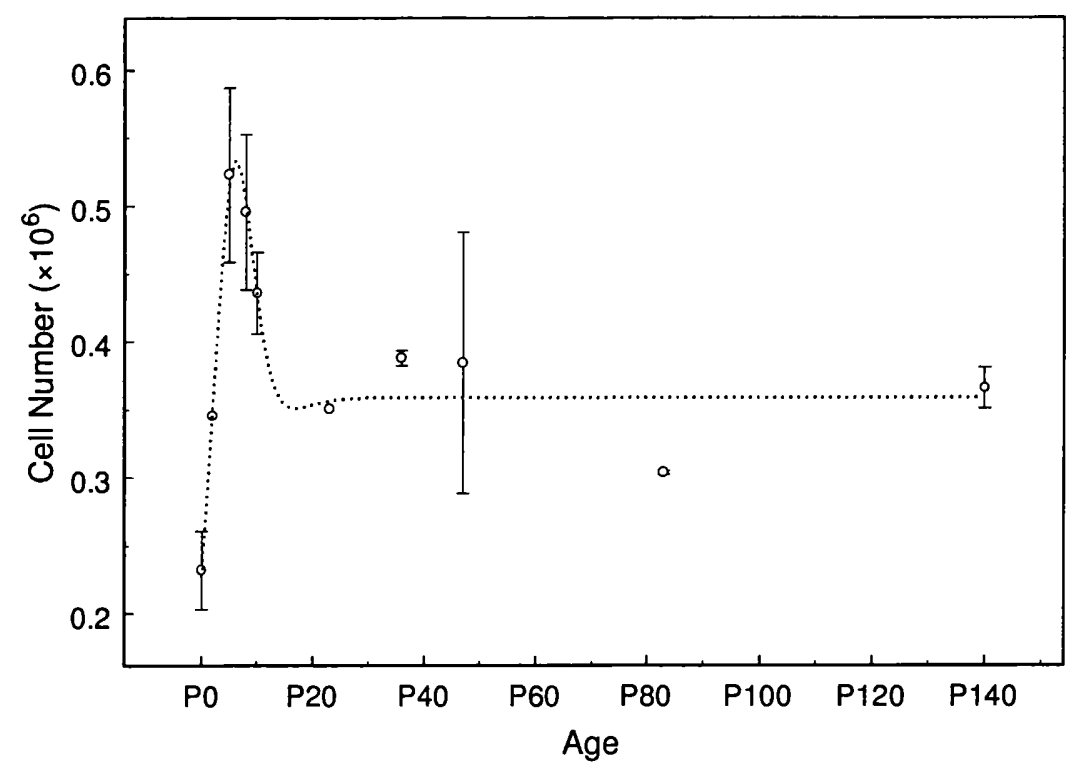

Figure 4.5 - Mean total cell numbers in the LGN calculated as the product of volume and density. Cell numbers peak around the end of the first postnatal week, and the adult number of cells is reached by the third postnatal week. 


\subsubsection{LGN cell size during development.}

The cross-sectional area of LGN cells was calculated at uniformly distributed sampling regions within a single section mid-way between the dorsal and ventral points of the LGN. The pooled data from all regions are shown in Figure 4.6a, where each point represents the mean area at the age indicated, together with the associated standard deviation.

During the first four postnatal weeks, cells of the LGN more than double in size, reaching their final adult size approximately during the eighth postnatal week (Figure 4.6a). The peak in the rate of cell growth occurs during the second week postnatal, where cross-sectional cell area is increasing at approximately $2 \mu \mathrm{m}^{2} /$ day (Figure $4.6 \mathrm{~b}$ ). An increase in the standard deviation of observations occurs with age, which may be attributed to differentiation of the cellular population into larger neurones and smaller glia. The drop in cell area between P47 and P140 is most probably due to the fact that only one animal was used in the calculation of P47 cell areas, and may not reflect the true value of the population.

\subsubsection{Distributions of cellular sizes within the LGN.}

The distribution of cell size with development is illustrated by the series of histograms in Figure 4.7. Superimposed on each graph is the mean cell area as shown in Figure 4.6a above. During the early period of development (E38 - P5), the distribution of cell sizes is very small, with little deviation about the mean value, indicating a uniform population of LGN cells. This is followed by a period where cell sizes become more diverse, which continues until maturity. At P140, two distinct populations are visible, the first tight cluster probably representing the smaller glial 
cells, while the second broad population probably represents neurones. The broad spectrum of this second group may reflect the differences in size between the various types of relay cells and interneurons (Guillery, 1966; LeVay and Ferster, 1977). Based on Guillery's (1966) classification of LGN cells, type 1 relay cells are generally the largest, followed by type 2 cells, interneurons (type 3), and finally the very small type 4 relay cells.
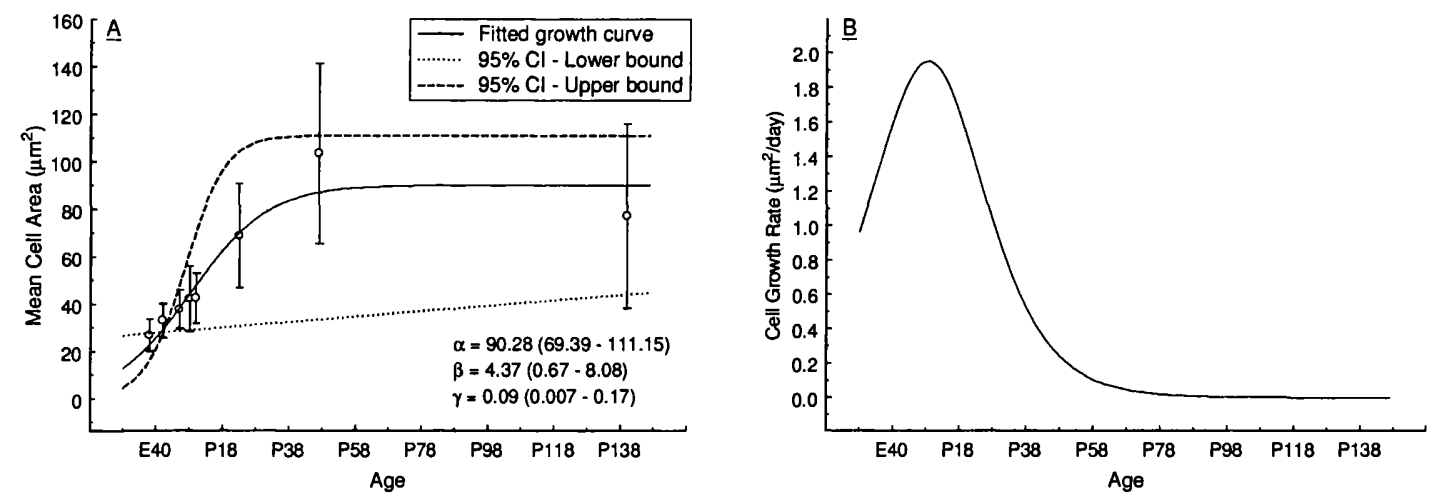

Figure 4.6 - (A) Mean LGN cell cross-sectional areas taken from one section mid-way between dorsal and ventral poles of the LGN, at uniformly sampled locations. Superimposed is a growth curve, and its derivative is shown on the right (B), which indicates the rate at which cell growth is occurring. The large standard deviations evident with age are attributed to the differentiation of the cellular population into neurones and glia. Estimates of the growth curve parameters are shown together with $95 \%$ confidence intervals.

\subsubsection{Comparisons with the thalamus.}

\subsubsection{Cell densities in LGN and thalamus - measurements from mid LGN.}

The 3D contour plots on the following pages show cell densities in the LGN and thalamus across a single section taken mid-way between the dorsal and ventral points of the LGN (Figures 4.8a-f). Higher cell densities are represented both in terms of greater height and red shading, lower densities are represented in blue. In order to indicate the position of the LGN on the contour map, a plane containing the outline of 
the nucleus is superimposed on top of each plot. The baseline of all plots is the mean number of cells counted in each sampling grid over the region of the thalamus. 

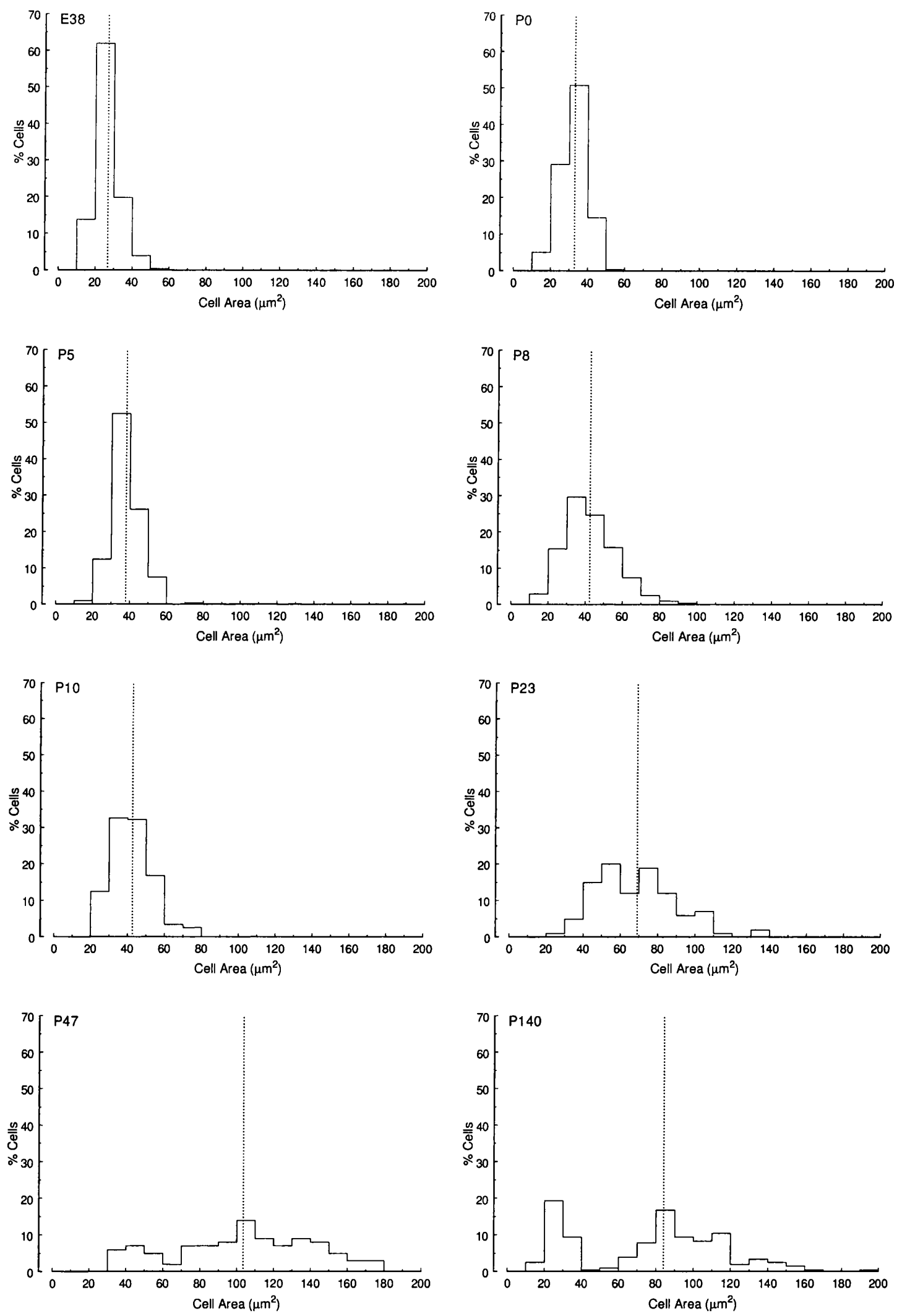

Figure 4.7 - Distributions of LGN cell sizes as measured from cross-sectional areas taken from one section mid-way between dorsal and ventral poles of the LGN, at uniformly sampled locations. The dotted line represents the mean cell area. At each age, data is pooled from 2 animals, with 10 cells measured from 10 sampling locations (total 200 cells/graph). 

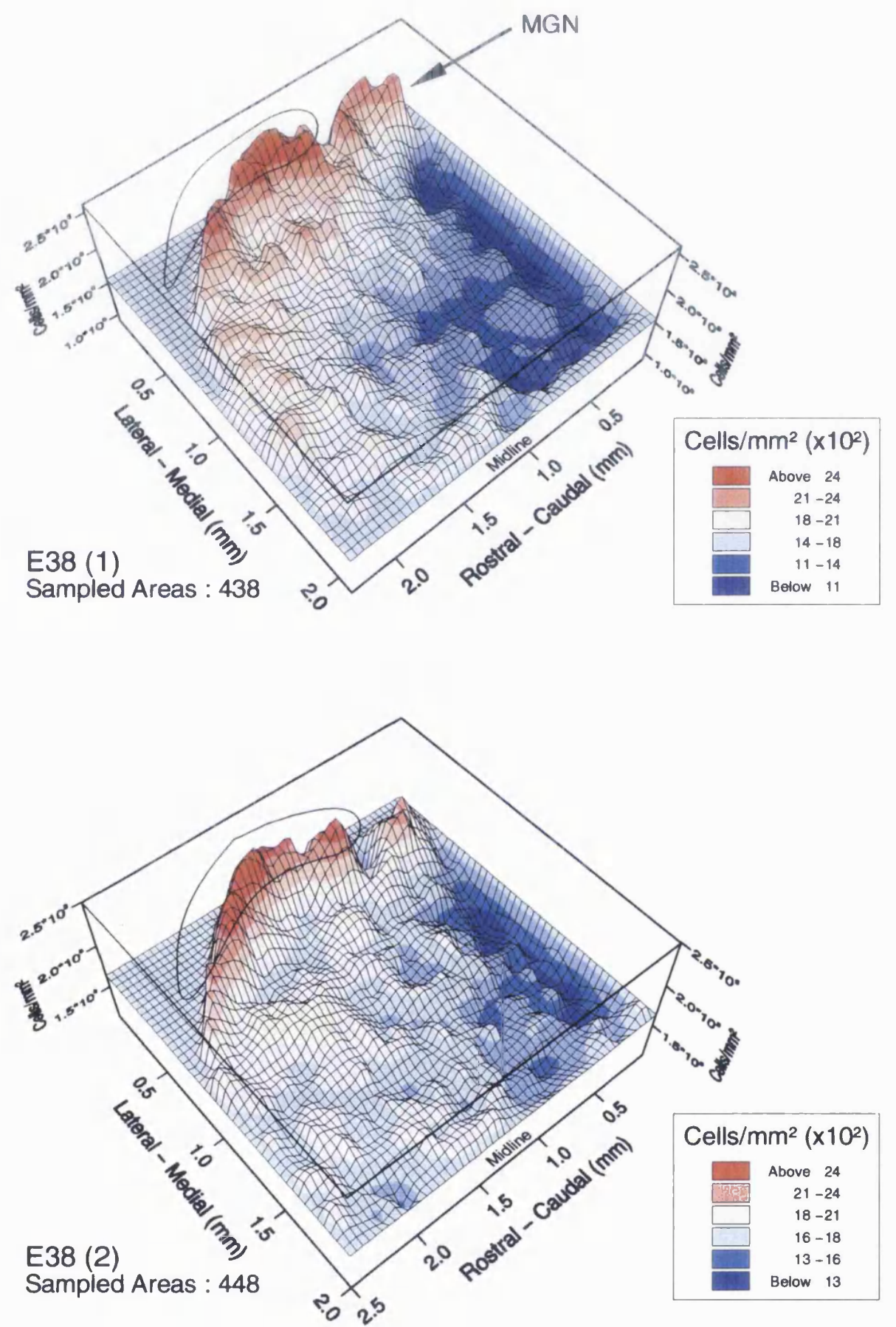

Figure 4.8a - Cell densities in the LGN and thalamus of two E38 animals as observed in a single horizontal section taken mid-way between the dorsal and ventral poles of the nucleus. Higher densities are shown in terms of both greater height, and red shading. Superimposed above the plot is a flat plane containing the outline of the LGN in order to indicate its position on the contour map. MGN: medial geniculate nucleus. 

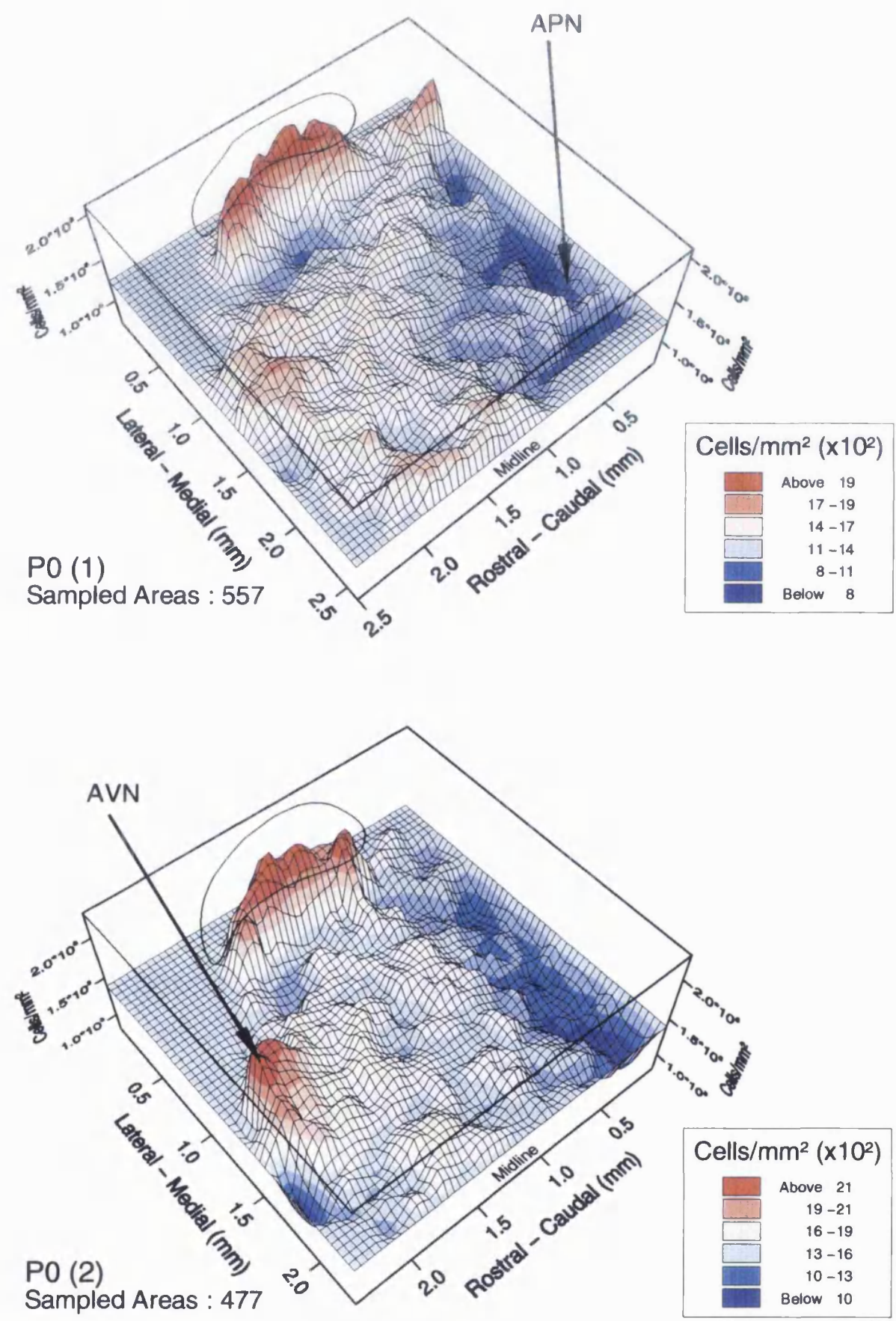

Figure 4.8b - Cell densities in the LGN and thalamus of two P0 animals as observed in a single horizontal section taken mid-way between the dorsal and ventral poles of the nucleus. Higher densities are shown in terms of both greater height, and red shading. Superimposed above the plot is a flat plane containing the outline of the LGN in order to indicate its position on the contour map. APN: anterior pretectal nucleus; AVN: antereoventral nucleus. 

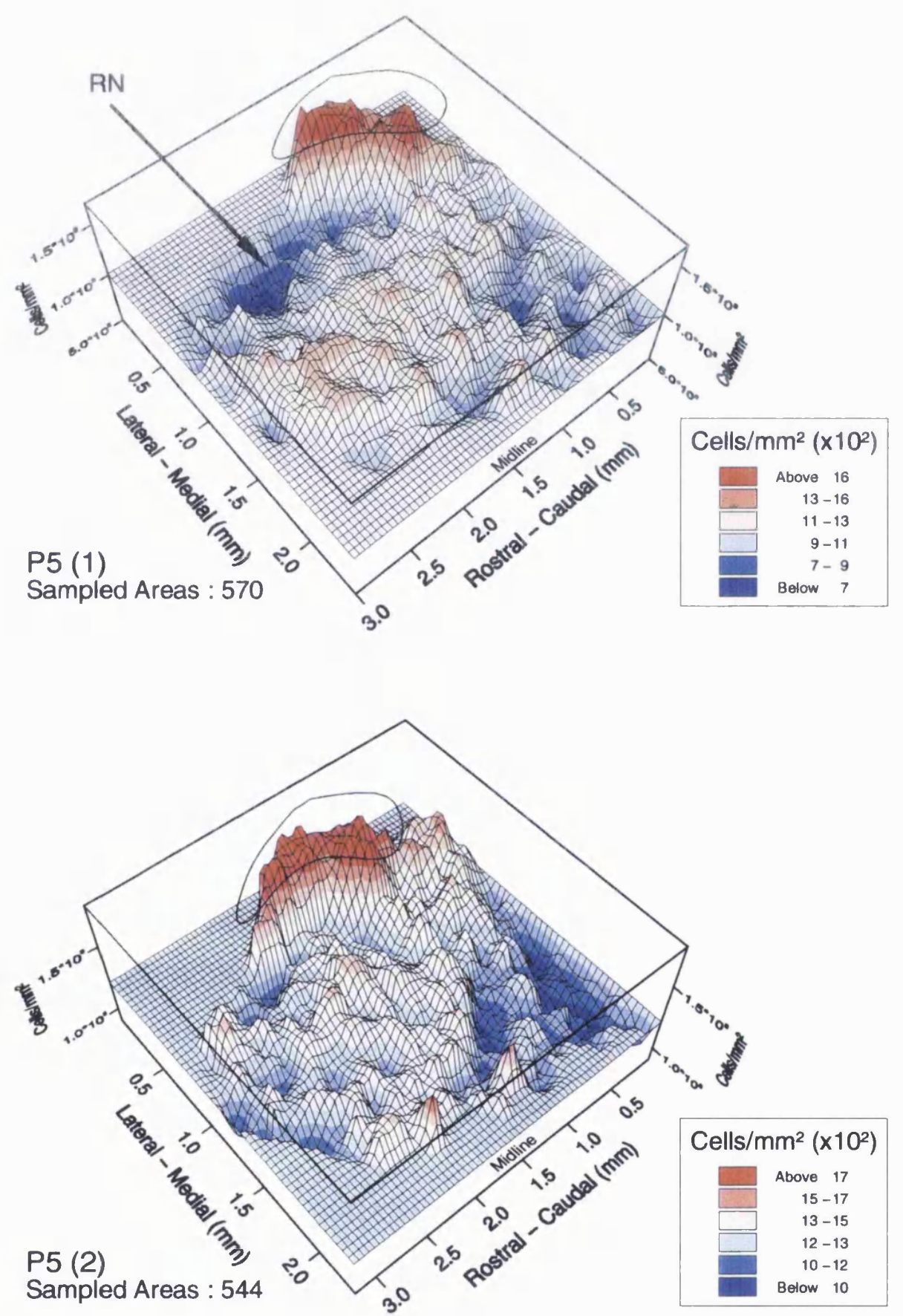

Figure 4.8c - Cell densities in the LGN and thalamus of two P5 animals as observed in a single horizontal section taken mid-way between the dorsal and ventral poles of the nucleus. Higher densities are shown in terms of both greater height, and red shading. Superimposed above the plot is a flat plane containing the outline of the LGN in order to indicate its position on the contour map. RN: reticular nucleus. 

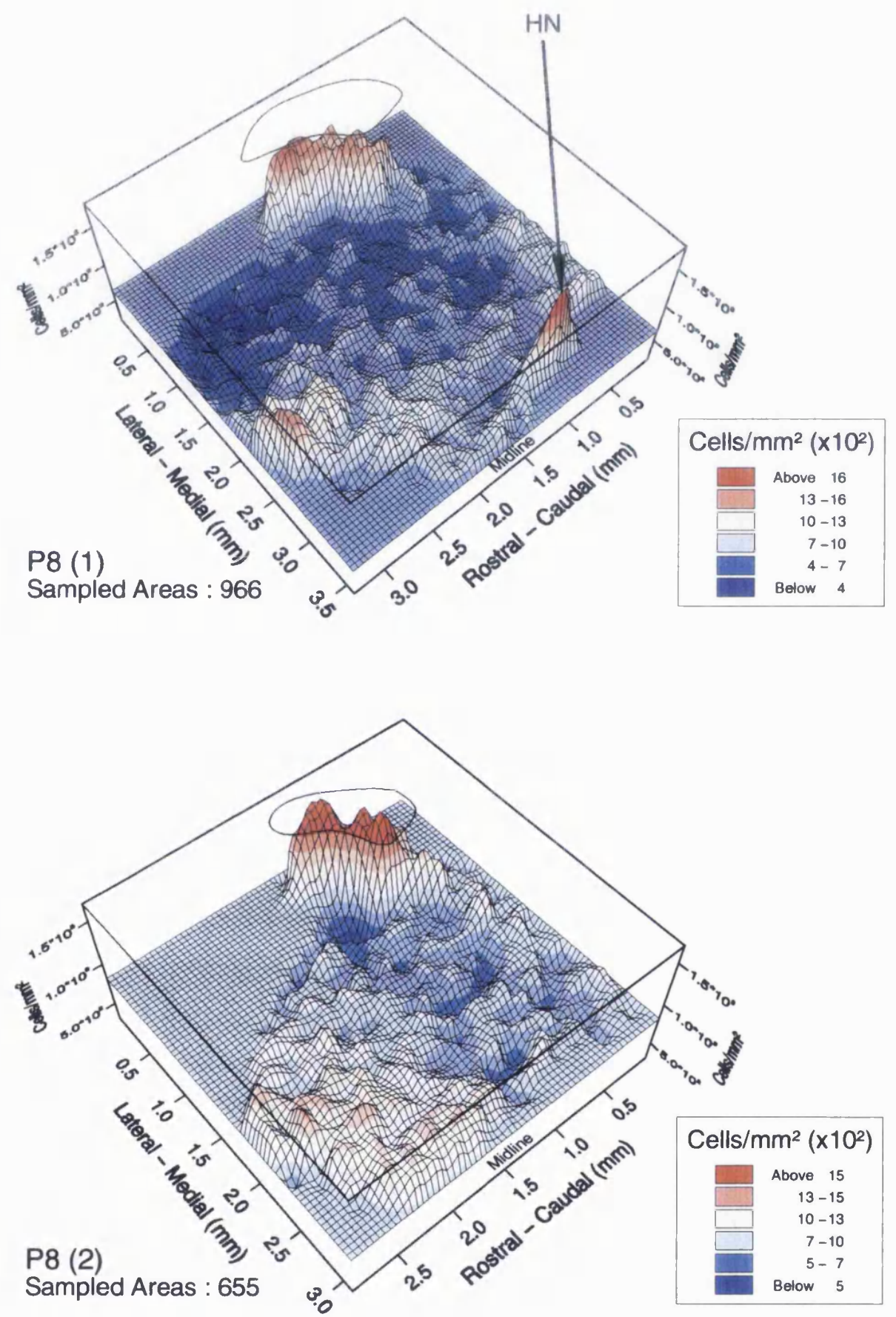

Figure 4.8d - Cell densities in the LGN and thalamus of two P8 animals as observed in a single horizontal section taken mid-way between the dorsal and ventral poles of the nucleus. Higher densities are shown in terms of both greater height, and red shading. Superimposed above the plot is a flat plane containing the outline of the LGN in order to indicate its position on the contour map. HN: habenular nucleus. 

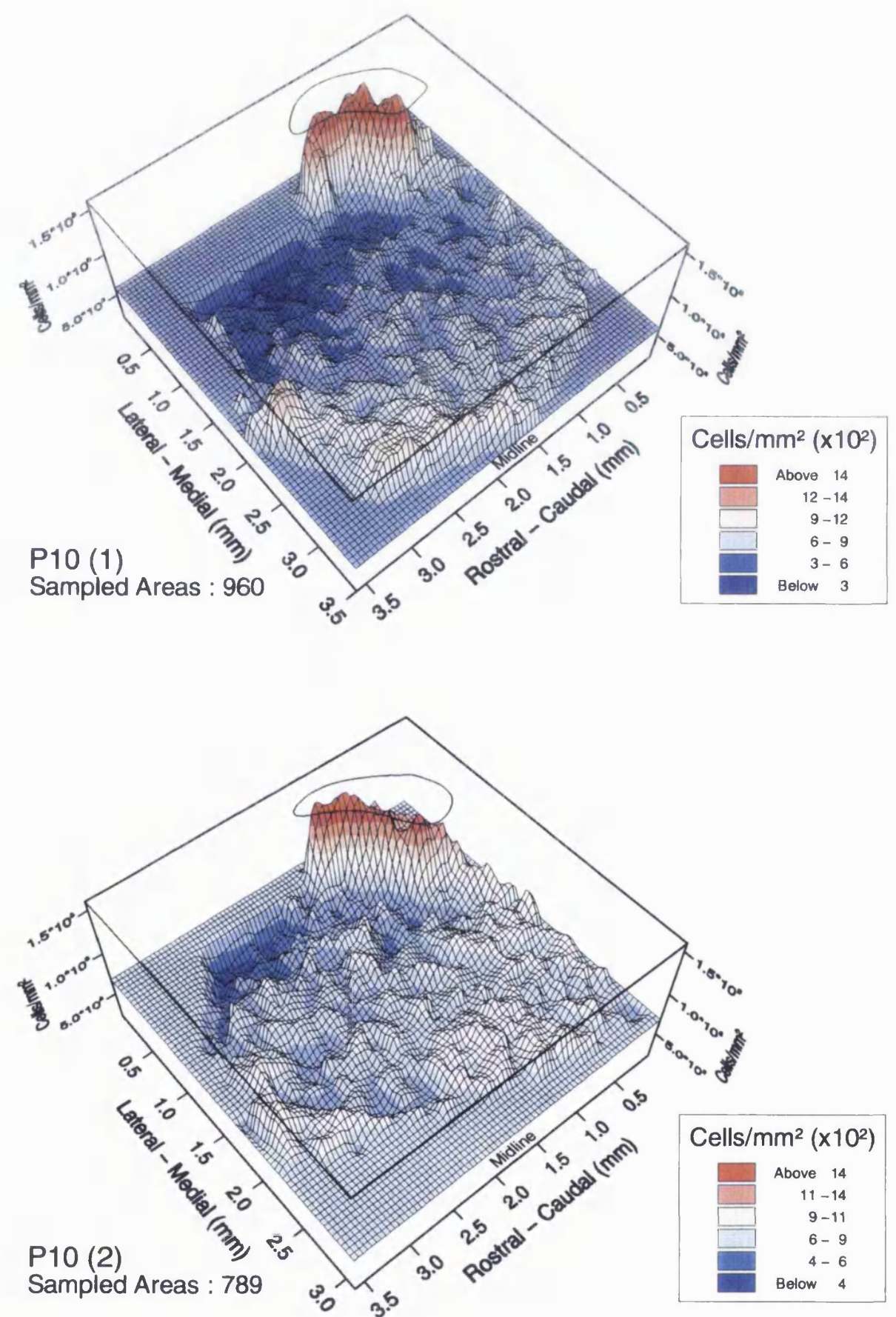

Figure 4.8e - Cell densities in the LGN and thalamus of two P10 animals as observed in a single horizontal section taken mid-way between the dorsal and ventral poles of the nucleus. Higher densities are shown in terms of both greater height, and red shading. Superimposed above the plot is a flat plane containing the outline of the LGN in order to indicate its position on the contour map. 

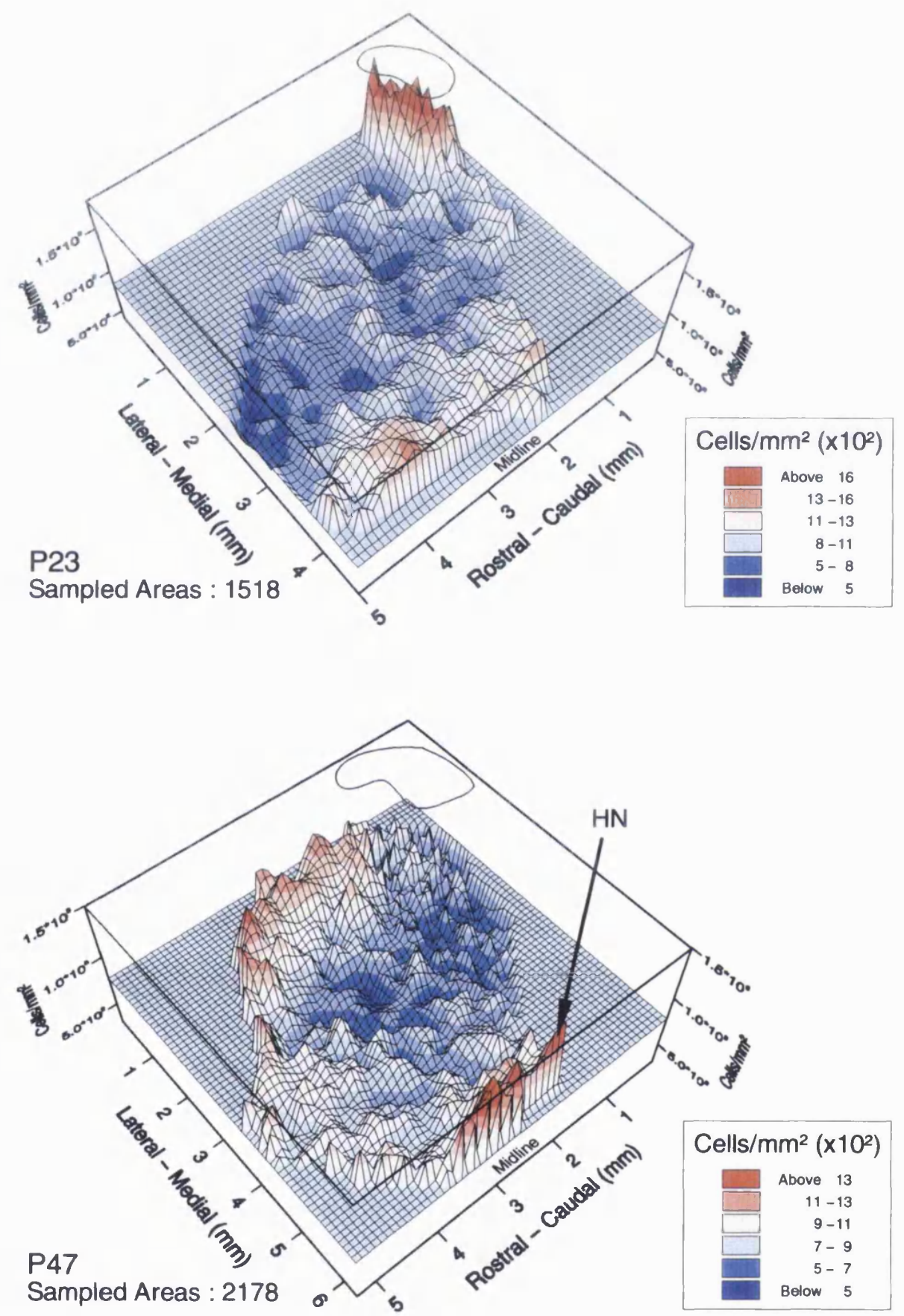

Figure 4.8f - Cell densities in the LGN and thalamus of P23 and P47 animals as observed in a single horizontal section taken mid-way between the dorsal and ventral poles of the nucleus. Higher densities are shown in terms of both greater height, and red shading. Superimposed above the plot is a flat plane containing the outline of the LGN in order to indicate its position on the contour map. HN: habenular nucleus. 
Examination of the plots reveals that the LGN has a consistently higher density of cells compared to other regions of the thalamus during development (Figures 4.8a-f). But by P47 this differential density has disappeared, and a relatively flat map is evident across the thalamus.

The plots for the E38 animals show a peak in cell density in the LGN, and also a peak in the medial geniculate nucleus (MGN, Figure 4.8a), the region immediately caudal to the LGN. The remainder of the thalamic region displays no other obvious peaks, simply a steady gradient of cellular density from caudal to rostral thalamus. The density of cells in the region of the MGN can be seen to diminish at progressive stages. At P0, it is still visible in one animal (Figure 4.8b, P0(1)). But 5 days later (Figure 4.8c), cell density is lower in the MGN than in the LGN. After the first postnatal week, cell density in the MGN is similar to the remainder of the thalamus (Figure 4.8d).

The rostro-caudal cell density gradient seen in the thalamus at E38 is also visible on the day of birth (Figure 4.8b). The figure also shows that although the gradients are similar, higher cell densities are observed in rostral regions at P0 in particular in the region of the antereoventral nucleus (AVN, Figure 4.8b). This is also the case for cell density in caudal regions (approximately in the area of the anterior pretectal nucleus, APN, Figure 4.8b). A similar cell density gradient is present in some of the older animals (e.g. P8, Figure 4.8d), but not as clearly as for these perinatal animals.

At P5, the region of the reticular nucleus becomes more clearly defined in terms of cellular density (RN, Figure 4.8c): a distinct decline in density is observed rostral to the LGN, and in a rostro-lateral region of the thalamus. This is also evident at later stages. 
The exception again is at P47 where an increase in density is observed (Figure 4.8f), although this may be due to the fact that the reticular nucleus has a high glial cell population, and may have adversely affected the counts.

Two of the animals used in this study (P8(1), P47) had extreme peaks of cell density close to the midline. These counts were from the region of the medial habenular nucleus (HN, Figures $4.8 \mathrm{~d} \& 4.8 \mathrm{f})$, and indicate that the sections analysed were either taken from a horizontal plane further dorsal than all the others, or that the plane of section was not perfectly horizontal.

\subsubsection{Measurements from further dorsal and ventral regions.}

The cell density plots shown in Figure 4.8a-4.8f taken from the LGNs midhorizontal plane are representative of the nucleus as a whole, as sampling planes dorsal and ventral to it show similar patterns (Figure 4.9). The data presented for P5, P8, and P10 animals, show consistently higher cell density in the LGN compared to the remainder of the thalamus.

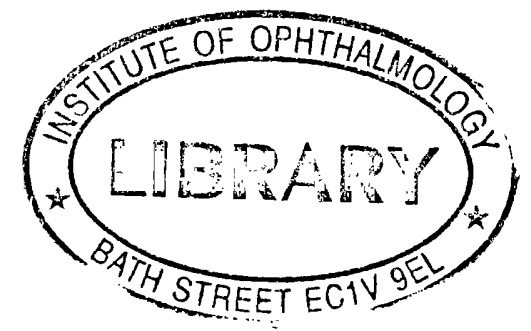



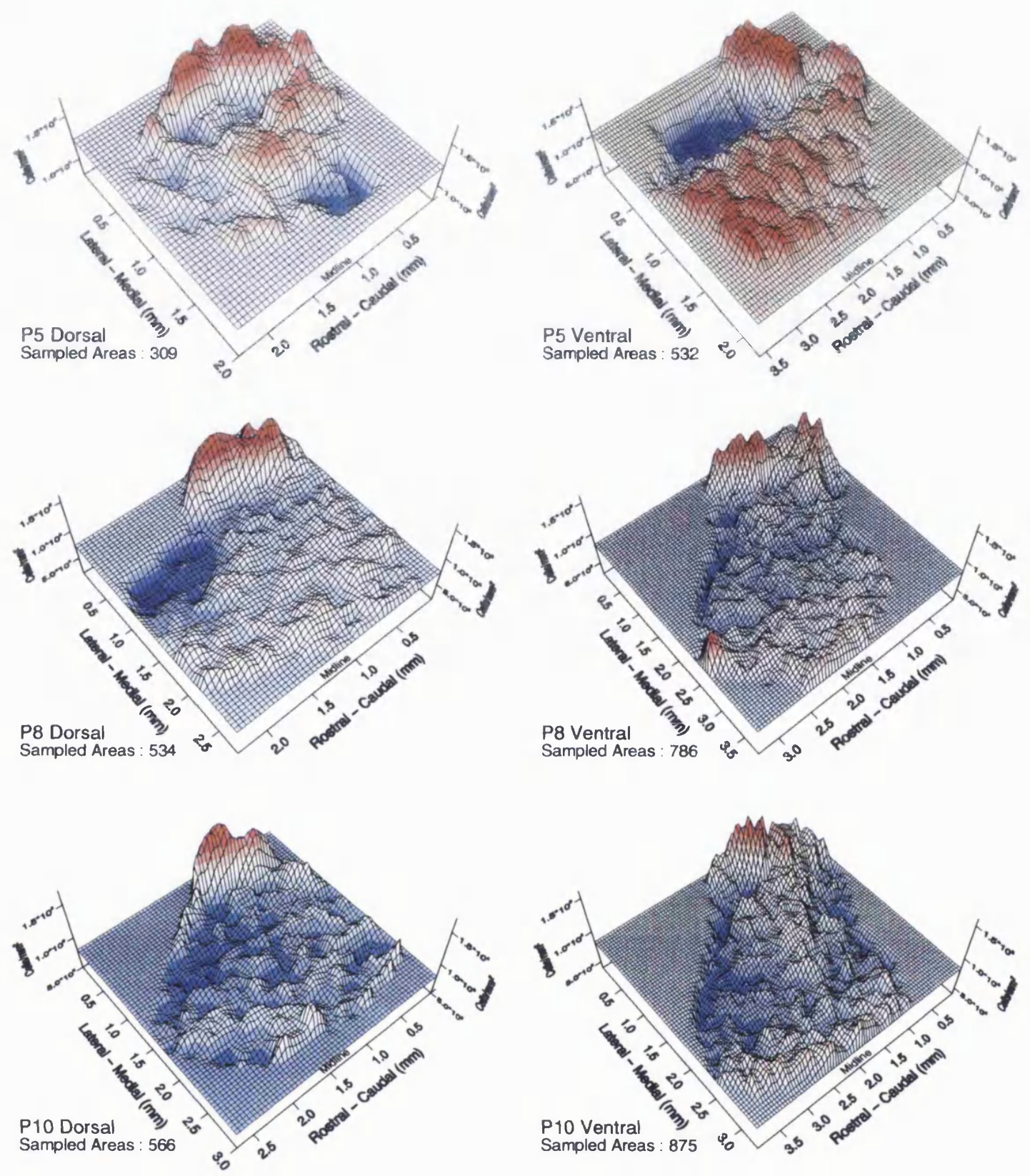

Figure 4.9 - Cell densities in the LGN and thalamus of three animals (P5, P8, P10) as seen in a single horizontal section taken from the mid-way between the dorsal pole and the mid-point, and the ventral pole and the mid-point. The plots illustrate that the mid-point sections of Figures 4.8a-f are probably representative of the remainder of the LGN in that the key feature of higher cell density in the LGN compared to the thalamus are also observed here.

\subsubsection{Cell sizes within the thalamus during development.}

Mean thalamic cell areas are shown in Figure 4.10a. For comparative purposes, the curve describing the growth in LGN cell area (Figure 4.6a) is superimposed on the 
data. The expansion in thalamic cells follows a similar pattern to that seen in the LGN, with the exception that cell areas are generally greater in the thalamus. Wilcoxon significance tests between the means of both populations at each age suggest that there is sufficient evidence beyond the $5 \%$ level to show that they are significantly different. This is true for all cases except P8. The peak in the rate of cell growth occurs at the same time in the thalamus and LGN. However, at this point, thalamic cells are increasing in area at a rate of approximately $3 \mu \mathrm{m}^{2} /$ day compared to $2 \mu \mathrm{m}^{2} /$ day in the LGN.
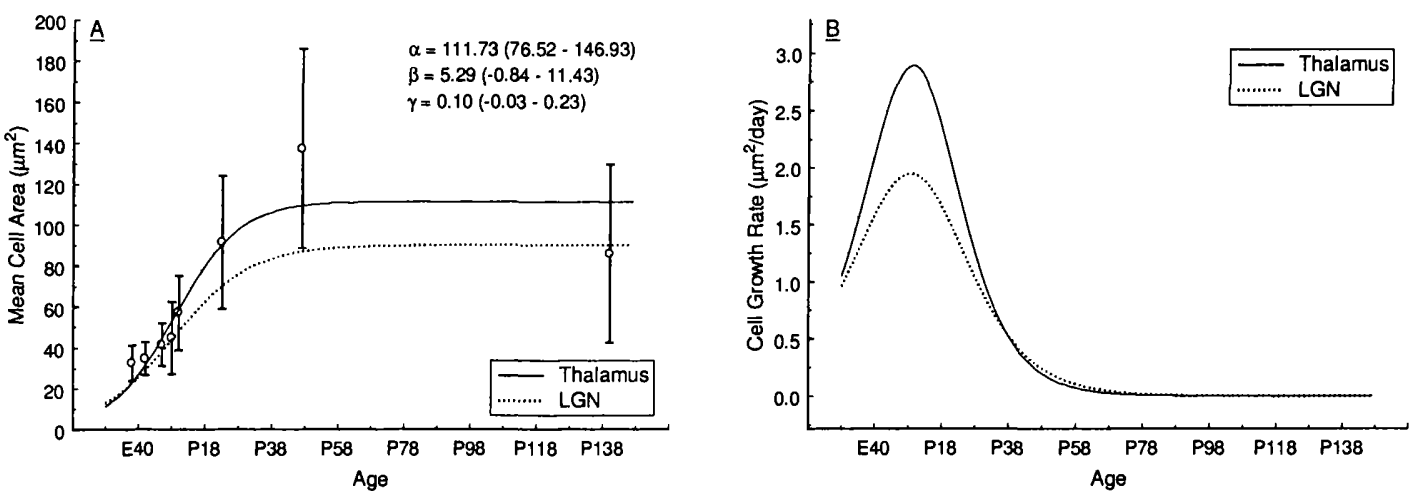

Figure 4.10 - (A) Mean thalamic cell cross-sectional areas taken from one section mid-way between dorsal and ventral poles of the LGN, at uniformly sampled locations. Superimposed is a growth curve (solid line; estimated parameters are shown together with 95\% confidence limits), and its derivative is shown on the right, (B), which indicates the rate at which cell growth is occurring. The growth curve derived for LGN cell cross-sectional areas as shown in Figure 4.6 has been included for comparative purposes.

\subsubsection{Distributions of thalamic cell sizes.}

Similar to Figure 4.7, Figure 4.11 shows the distribution of thalamic cell area by age. The mean thalamic cell area is superimposed on each graph. In comparison with LGN cell sizes, thalamic cells show a slight increase in distribution range at each age. Despite this, similar distributional patterns are observed in the thalamus and LGN: 
early in development, distributions show a uniform population of cells, and by adulthood, two separate populations of cells are observed. 

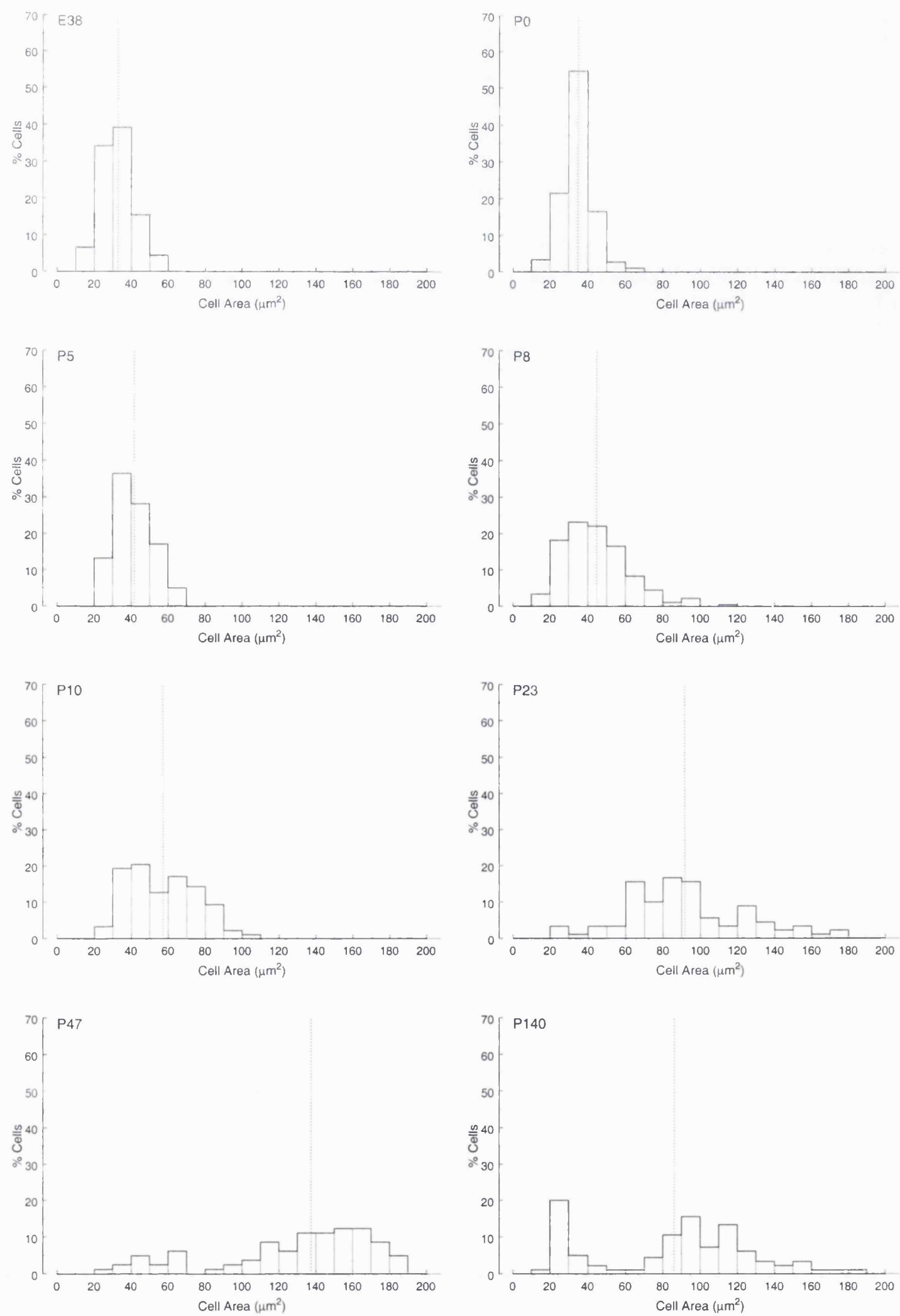

Figure 4.11 - Distributions of thalamic cell sizes as measured from cross-sectional areas taken from one section mid-way between dorsal and ventral poles of the LGN, at uniformly sampled locations. The dotted line represents the mean cell area. At each age, data is pooled from 2 animals, with 10 cells measured from 10 sampling locations (total 200 cells/graph). 


\subsubsection{Spatial distributions of cells.}

\subsubsection{Distribution according to density.}

To examine cell density in the LGN in greater detail, data for cell counts in the nucleus shown in Figure 4.8a-4.8f are replotted at a higher scale below (Figure 4.12). For clarity of illustration, these are shown as two dimensional contour maps, and each is plotted on an individual scale to highlight the spatial distributions. No major changes in density across the nucleus are evident over the period examined. Contour lines (indicated by changes in grey-level) tend to follow the longitudinal axis of the nucleus (i.e. parallel to the lateral edge), indicating an increase in density towards its medial regions. In addition, rostral areas tend to show higher cell densities, while the very lateral areas (the region of the $\mathrm{C}$ laminae) show lower densities in comparison.
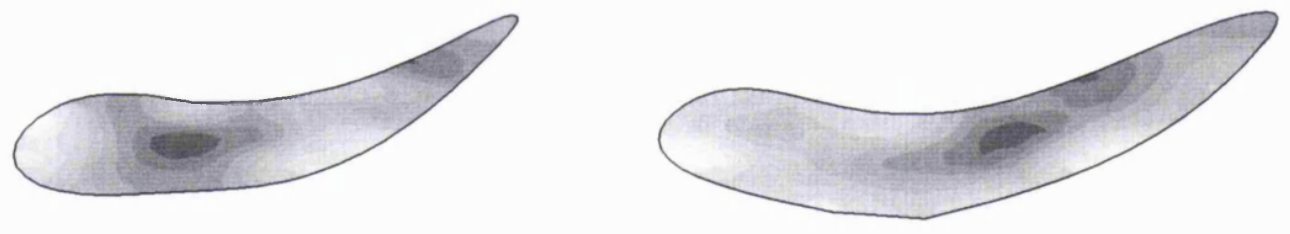
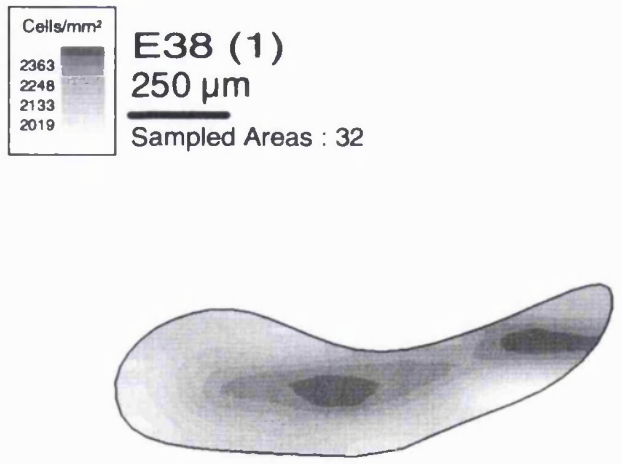
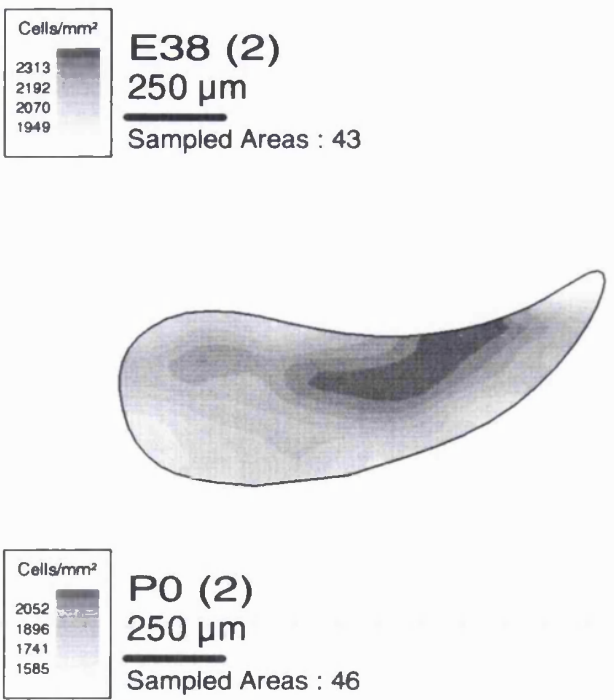

$\mathrm{PO}(2)$

$250 \mu \mathrm{m}$

Sampled Areas : 46

Figure 4.12 (Continued overleaf) - Variations in cellular density in the ferret LGN at the ages indicated. The data derives from that shown in Figure 4.6, but using a larger scale. Higher cell density is indicated in terms of darker shading. 

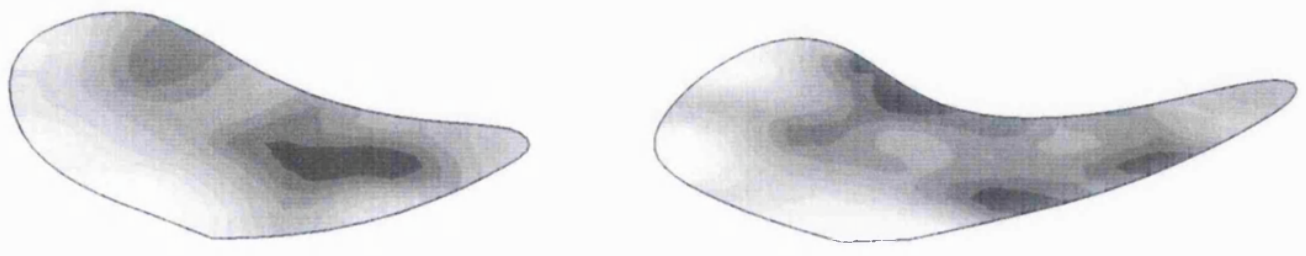

\begin{tabular}{l|l}
$\begin{array}{ll}\text { Celis/mm² } \\
1499 \\
1388 \\
1387 \\
1166\end{array}$ & $\begin{array}{l}\text { P5 }(1) \\
250 \mu \mathrm{m}\end{array}$ \\
\hline
\end{tabular}

\begin{tabular}{l|l}
$\begin{array}{ll}{ }_{1600} \\
1592 \\
1524 \\
1455 \\
145\end{array}$ & $\begin{array}{l}\text { P5 (2) } \\
250 \mu \mathrm{m}\end{array}$ \\
\hline Sampled Areas : 57
\end{tabular}
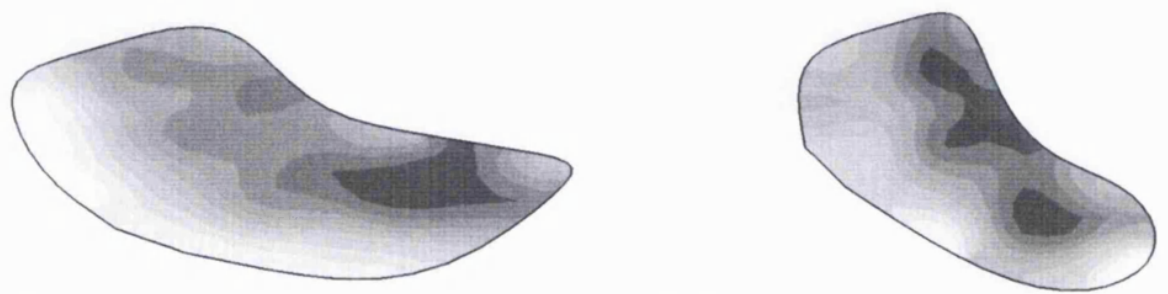

\begin{tabular}{|l|l|}
\hline $\begin{array}{l}\text { Colls } / \mathrm{mm}^{2} \\
1302 \\
1137 \\
972 \\
806\end{array}$ & P8 (1) \\
\hline & $250 \mu \mathrm{m}$ \\
\hline
\end{tabular}

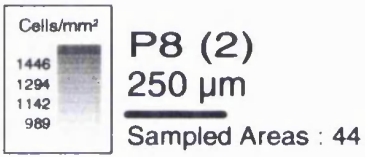
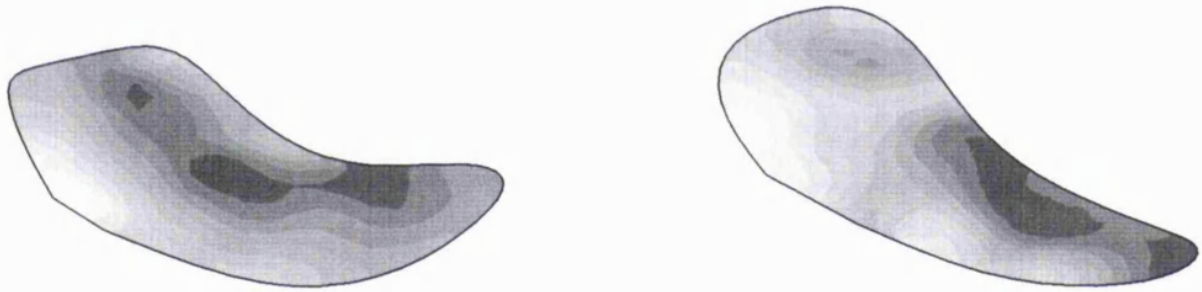

\begin{tabular}{l|l}
$\substack{1331 \\
117 \\
1023 \\
8 \otimes 9}$ & P10 (1) \\
\hline Sampled Areas : 55
\end{tabular}

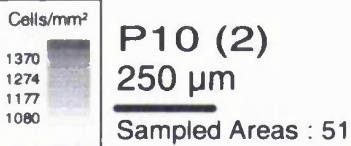
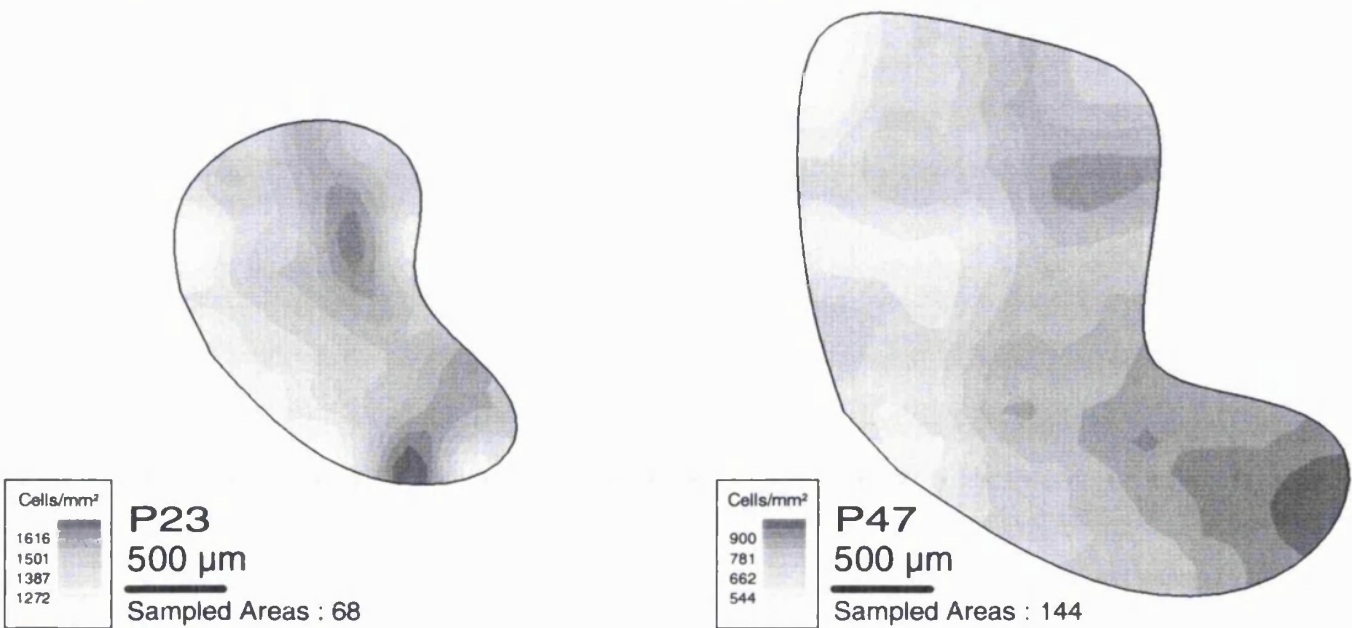

Figure 4.12 (continued) - Variations in cellular density in the ferret LGN at the ages indicated. 


\subsubsection{Distribution of cells according to size.}

The data from Section 4.3.2.3 were employed to examine the distribution of cell sizes within the LGN with respect to their location. Figure 4.13 shows contour plots of cell size within different regions of the LGN. Darker shading indicates larger cells. The general trend is the presence of larger cells in the caudal (binocular) area of the nucleus, and smaller cells in the rostral (monocular) regions. 

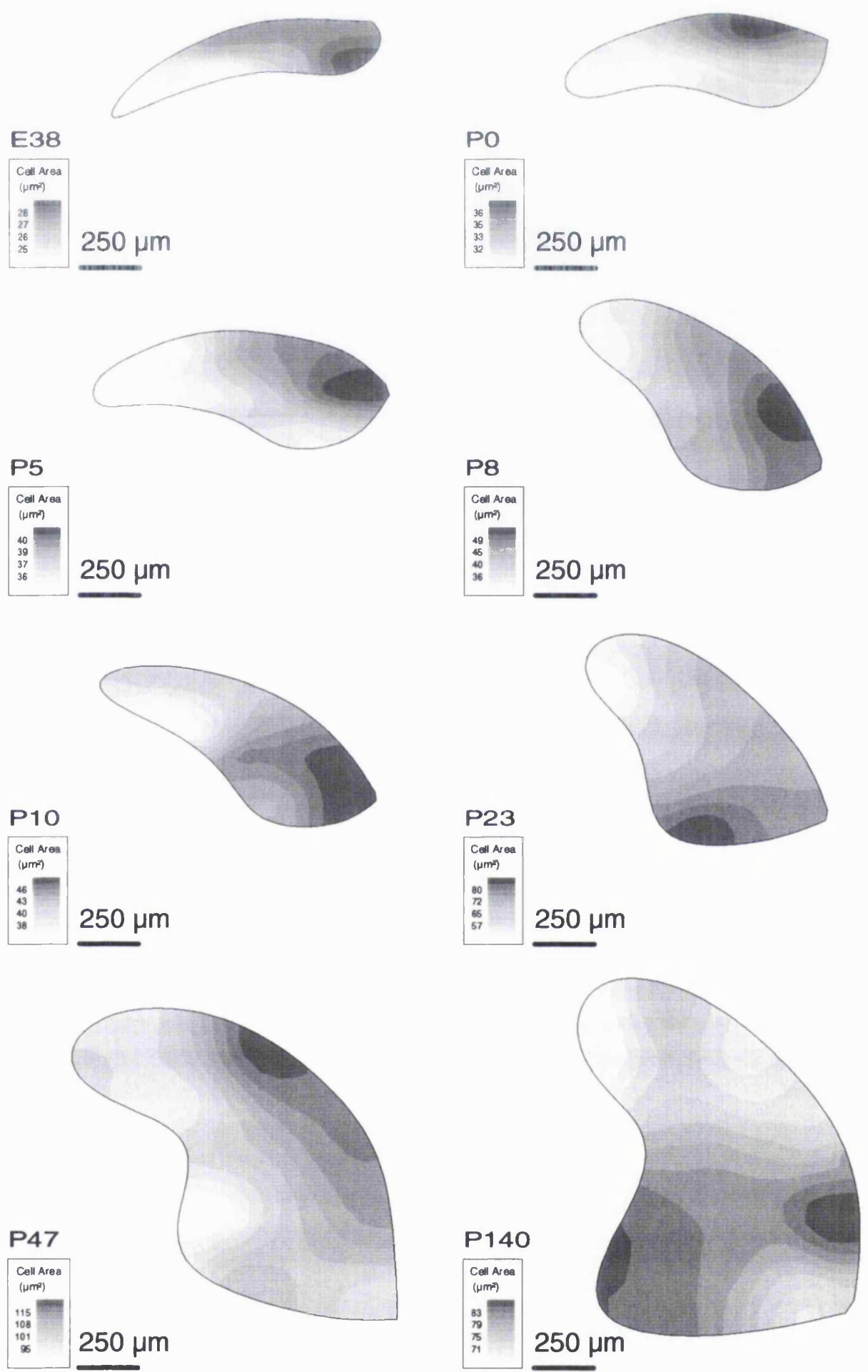

Figure 4.13 - Spatial distributions of cell size in the LGN at progressive stages of development. Darker shading represents larger cells. Plots show averaged data, i.e. data collected at corresponding positions shown in Figure 4.1b have been averaged. The general trend is the presence of larger cells in the caudal (binocular) area of the nucleus, and smaller cells in the rostral (monocular) regions. 


\subsection{Discussion.}

A stereological analysis has been undertaken and data are provided on nuclear volume, cell density, and cell numbers in the developing LGN. In addition, the relative distribution of cell densities has been explored. Data have also been provided on cell sizes during development.

Changes in LGN volume, cell density and cell number are consistent with data from other species (Elgeti et al., 1976; Villena et al., 1989). Volume increases substantially during the first six weeks, while cellular density falls over similar postnatal periods. Total cell numbers rise sharply between birth and the latter part of the first postnatal week, and decline thereafter, reaching the adult value during the early part of the fourth postnatal week.

No major changes in the variation of cell density across the LGN can be demonstrated during development with the methods employed here, but higher densities are generally observed on the medial aspects of the nucleus, and lower densities on the lateral aspect. Examination of thalamic and LGN cell densities reveals that the LGN has a consistently higher cell density compared to other thalamic regions during development. This persists until the animal reaches maturity, when densities become approximately equal.

Both LGN and thalamic cells reach their adult sizes approximately eight weeks postnatal, although thalamic cells are larger, and have a higher growth rate. Within the LGN, there is a general trend for larger cells to occupy the caudal (binocular) area of the nucleus, while smaller cells occupy rostral (monocular) regions. The presence of different size cells in these regions could account for the differences in their respective 
sizes, the binocular region being generally broader that the monocular when seen in cross section.

\subsubsection{Contributors to LGN growth.}

A twenty-fold increase in LGN volume is observed between birth and adulthood. This is larger than that observed in that rat (eleven-fold increase, Villena et al., 1989), and substantially larger than in the cat (six-fold increase, Elgeti et al., 1976). The cat and ferret have been shown to have similarities in the timing of developmental events when expressed on a post-conception scale (Robinson and Dreher, 1990). The cat is born 65 days post-conception, which is equivalent to postnatal day 23 in the ferret. The increase in ferret LGN volume between this age and maturity is reduced to approximately four-fold, and is thus comparable with that of the cat.

Data presented here provide an indication of the volume of the LGN occupied by cell bodies. By making the assumption that each cell is spherical, a simple calculation yields the volume of cell bodies at each developmental stage. As shown in Figure 4.14, the product of these volumes with the number of cells observed at each respective age provides an approximate measure of the volume of the LGN occupied by cell bodies.

Between P0 and P10, the ratio of the total volume of LGN cell bodies to nuclear volume is fairly constant at approximately $5: 1$. Hence at this stage, the nucleus is expanding at a similar rate to the space occupied by its cellular population. The observed reduction in total cell numbers towards the end of the first postnatal week is complemented by the peak in the rate of cell growth during the same period. Other factors must make up the remaining space, including dendritic field size, retinal ganglion cells terminal field arbor, and corticogeniculate axon arbors. Between P10 
and P23, the ratio has increased to approximately 7:1, and since soma size and LGN volume increase by similar amounts, other non-somatic factors must be involved. Sutton et al (1991) have demonstrated that there is a substantial increase in the branching density of dendritic trees in the ferret during this period, and this could account for the increased ratio between total cell body and LGN volume. By adulthood, the ratio has increased more than two fold to approximately 15:1, again most probably due to the general increase in the volume of neuropil. This has been reported to be the case over a similar time period in the cat (Weber and Kalil, 1987).

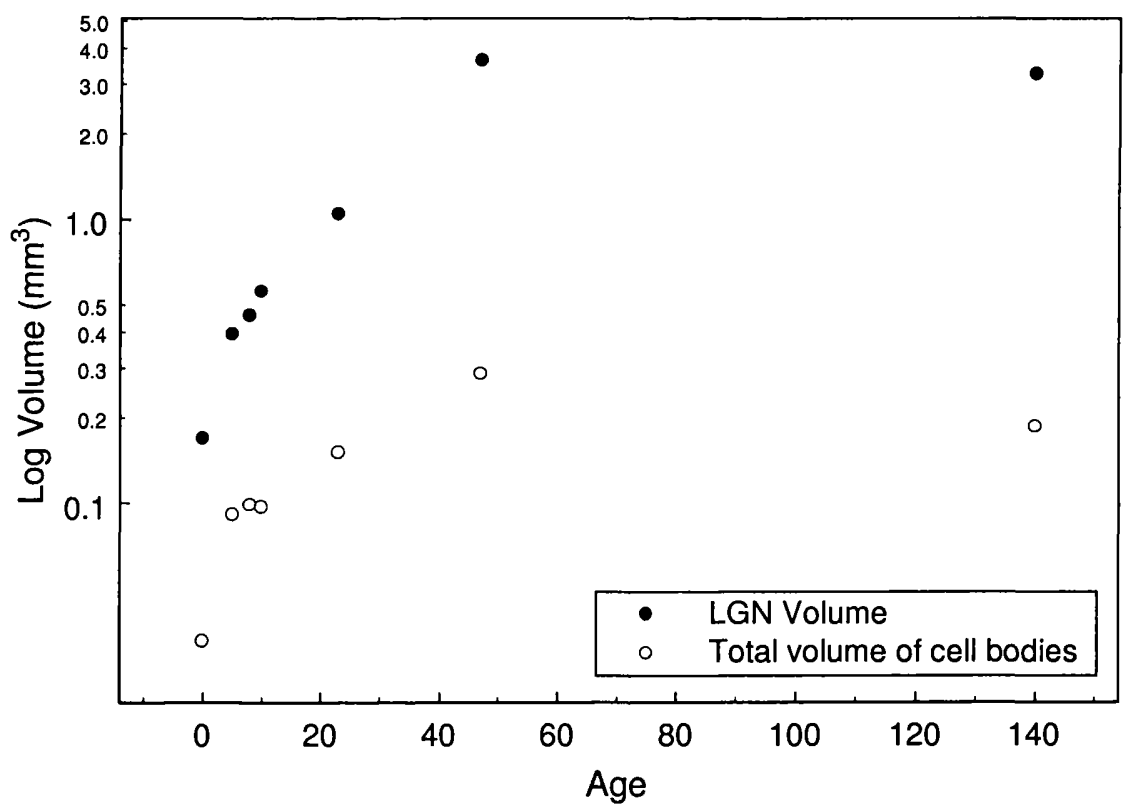

Figure 4.16 - Plot of the volume of the LGN (as shown in Figure 4.3a) and the sum of the volume of cell bodies within the LGN. Total cellular volume is an approximate measure, and was calculated as the product of cell number and cell volume, and assumes that all cells are spherical. Note, the volume is plotted on a logarithmic scale.

Kalil (1978) suggested that the late proliferation of glial cells, as shown by Elgeti et al (1976), makes an important contribution to the postnatal growth of the cat LGN. This could be the case here, although no data is presently available on gliogenesis in the ferret LGN. However since gliogenesis occurs relatively late in the cat (after 
completion of neurogenesis), it may well account for the increased ratio between LGN volume and cell body volume described above. One other possible contributor to the growth of the LGN could be the myelination of fibres both entering and leaving the nucleus. Moore et al (1976) have shown that myelination in the cat is a prolonged process which begins soon after birth, and continues for approximately four weeks, although no comparable data is available for the ferret.

\subsubsection{Differences between $L G N$ and thalamus.}

The data presented in Section 4.3.3 have shown a consistent difference in the density of cells between the LGN and the remainder of the thalamus. Between E38 and P23, cell density is higher in the LGN, and falls to a level seen in the thalamus between P23 and P47. This is reflected by the clear identification of the LGN histologically, at a time when no other thalamic nuclei in the same plane of section are cytologically well defined.

The most probable reason why there is a drop in LGN cell density between these periods is due to the stabilisation of cell numbers while nuclear volume is still increasing. Furthermore, crude measurements between P23 and P47 of the areas of LGN and thalamus in which the counts were performed show a $73 \%$ increase in the area of the former, compared to a $36 \%$ increase in the latter. If thalamic cellular populations have stabilised as in the LGN, then the increased area could account for the phenomenon observed.

It is possible that the data is representative of the period of gliogenesis. Studies in both cat (Elgeti et al., 1976) and rat (Biesold et al., 1976) show that gliogenesis in the LGN continues into postnatal life. Since no distinction was made here between 
neurones and glia, the observed differences could therefore be accounted for by the increase in the glial cell population. This does assume that gliogenesis in the remaining thalamus is either not as proliferative or has stabilised before the LGN.

\subsubsection{System Matching.}

A substantial reduction in cell number in the LGN between the first and third postnatal weeks has been demonstrated here. This is consistent with the phenomenon of cell death. Cowan (1973) proposed cell death as a mechanism for the matching of neuronal numbers between two populations. In the LGN, cell numbers fall from approximately 500,000 at P5, to approximately 350,000 at P20 - a 30\% decrease. In the ferret, retinal ganglion cell numbers fall from approximately 155,000 at P3, to approximately 100,000 at P6 (Henderson et al., 1988), although the period of ganglion cell loss has been shown to be more prolonged (Cucchiaro, 1991). Nevertheless, the reduction is approximately $35 \%$, and is comparable to that seen in the LGN. Here, it has not been possible to directly compare neuronal numbers between the retina and LGN, since no differentiation between neurones and glia was made when counting. However, the fact that cell numbers reduce by proportionate amounts indicates a possible relationship between the two populations.

\subsubsection{Summary.}

The data presented in this chapter illustrate that the cellular population of the LGN only partially contributes to the substantial growth of the nucleus. It is envisaged that a number of other elements such as axon arbors, dendritic arbors, glial cell population, and myelination are additionally implicated in LGN growth. The relative distributions of cellular densities as analysed here have not revealed any particular 
patterns which could be attributed to the changing shape of the LGN. In contrast, the spatial distribution of soma according to size could be responsible for the different cross-sectional areas of the binocular and monocular regions of the nucleus. 


\section{Three-dimensional growth dynamics in the ferret LGN.}

\subsection{Introduction.}

During development, the LGN displays distinct changes in shape and orientation, but to date, analysis of these events have only been qualitative (monkey: Rakic, 1977; cat: Kalil, 1978; ferret: Linden et al., 1981). In the late prenatal ferret, the LGN sits on the lateral and dorsal margin of the diencephalon. In the horizontal plane it has an appearance similar to a curved elongated ellipse, often described as a "comma-shaped" structure (Linden et al., 1981), with its long axis lying along the rostro-caudal plane. By adulthood, the nucleus has transformed into a thick L-shaped structure, with its long axis displaced medio-laterally. These changes are gradual, and can be identified during periods that overlap with the changing patterns of retinal innervation.

A number of events may influence the structural development of the LGN, for example retinal ganglion cell innervation or the dendritic development of its constituent cellular population. The question of whether there is any significance to these changes in shape, or whether they result from the physical dynamics of the developing brain, remains unanswered. In an attempt to address these questions, this chapter aims to 
provide some base data concerning the changing three-dimensional shape of the developing LGN, and modelling techniques described in Chapter 2 are employed to map the transformations that occur with development. However, the question as to whether changes in LGN shape are statistically significant over time cannot be addressed due to the low numbers within each age group.

In addition to the changes that occur in LGN shape during development, Kalil (1978) and Rakic (1977) have described a rotation of the nucleus in cat and monkey. The studies used different methods to define rotation: Kalil (1978) defined it based on the anatomical borders of the nucleus, and their relationship with the medial geniculate nucleus and cerebellum; Rakic (1977) on the other hand defined rotation solely on the basis of the axis of cell generation within the LGN. Kalil stated that rotation occurs mainly in the sagittal plane, while Rakic described a rotation of the coronal plane (Figure 5.1). Furthermore, Shatz (1984) has also described a prenatal rotation of the cat LGN in the horizontal plane.

In this chapter, the issue of rotation is addressed in a number of different ways: cross-sections in more than one plane are taken from models of mean LGN shape in order to assess rotation based on two-dimensional outlines of the nucleus, and global rotations of the nucleus are examined in three dimensions. It is important to determine the nature and degree of LGN rotation, since they could underestimate or exaggerate any measurements made on the precise changes in shape of the nucleus between different ages. In light of this analysis, temporal and spatial characteristics of shape change are reviewed. 

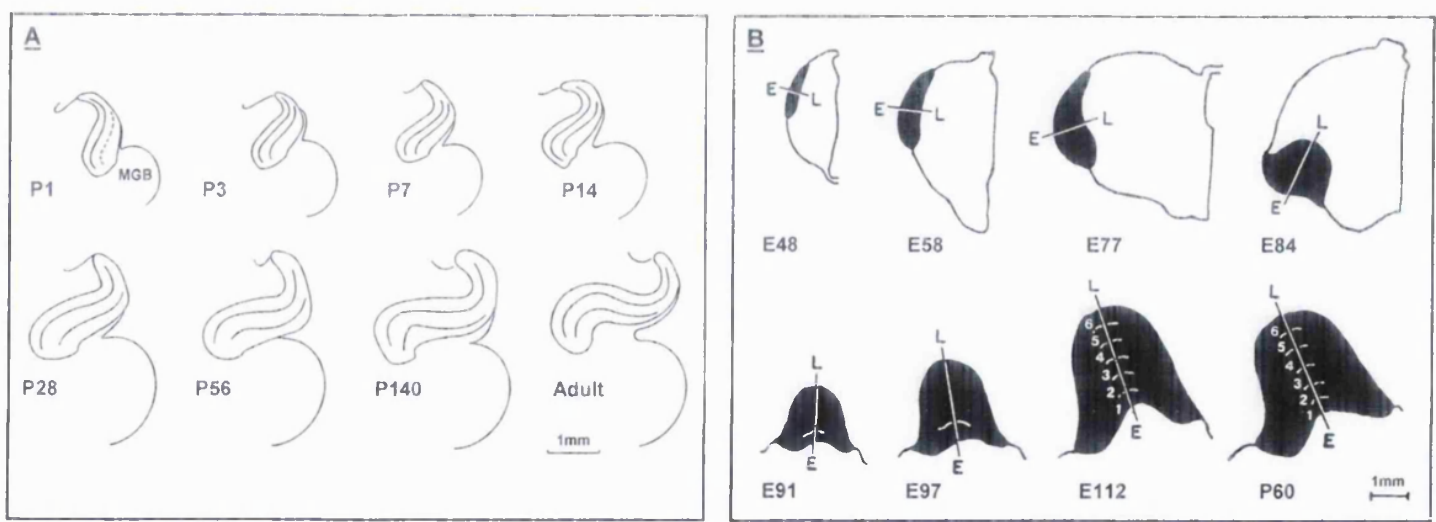

Figure 5.1 - Rotation of the LGN in the cat (A, sagittal sections, from Kali1, 1978) and monkey (B, coronal sections, from Rakic, 1977). Dorsal is towards the top of both figures, rostral towards the left in $\underline{A}$, and lateral towards the left in $\underline{B}$. In $\underline{A}$, the lines within the nucleus indicate the interlaminar zones. In $\underline{B}$, the axis E-L indicates the orientation of the early to late gradient of neurogenesis, and the numbers 1-6 in E112 and P60 indicate the position of the layers which emerge in late development.

\subsection{Methods.}

\subsubsection{Generation and analysis of $3 D$ models.}

Three dimensional reconstructions of LGN's were created for animals ranging between E38 and P140 as described in Section 2.5.2, and surface models were fitted to these individual reconstruction datasets. The number of models generated at each stage is shown in Table 5.1.

\begin{tabular}{ccccccccccccc}
\hline Age & E38 & P0 & P2 & P5 & P8 & P10 & P23 & P36 & P47 & P83 & P111 & P140 \\
\hline$n$ & 2 & 4 & 2 & 3 & 3 & 4 & 1 & 2 & 2 & 2 & 2 & 1 \\
\hline
\end{tabular}

Table 5.1 - Number of three-dimensional models of LGN structure created at each developmental stage.

Computationally, the time taken to construct each model was dependant upon animal age and therefore the size of the LGN. But also it depended on the type of computer creating the model. Models of the LGN from animals killed at P10 and 
younger were created on an IBM compatible PC running the Linux operating system. The software would generally take between four and eight days to optimally map the individual points from the original reconstruction dataset to the surface of a unit sphere, and thereafter calculate the spherical harmonic functions. LGN models from older animals (> P10) were created on a Cray $\mathrm{J} 90$ multiprocessor system ${ }^{1}$, where a solution was generally found in approximately 24 hours.

The mean shape of the LGN at progressive stages was calculated by averaging corresponding spherical harmonic coefficients of each surface model. These models were used to calculate the volume of the nucleus by double integration over the surface. This process is conceptually similar to calculating the area beneath a curve using integration.

Shape change may be considered as the difference between shapes at successive ages, and a measure of difference between shapes can be established by calculating the Euclidean distance (i.e. the straight line distance) between them. This measure is often referred to as a measure of dissimilarity (or a dissimilarity index), and is calculated as the sum of squared differences between corresponding spherical harmonic coefficients. To establish the temporal nature of LGN growth, dissimilarity measurements were calculated, and all were made relative to the youngest animal (E38). The resulting data provide a measure of growth, and for this reason, logistic (or growth) functions are fitted (see Section 2.6). The E38 animal was chosen as a starting point since it is known that at this stage, crossed and uncrossed RGC fibres are completely mixed

\footnotetext{
${ }^{1}$ A fixed amount of CPU time was allocated on the Cray J90 at the Rutherford Appleton Laboratory, Oxfordshire, and this was sufficient for creating the models for the older animals only.
} 
within the nucleus (Linden et al., 1981). In addition, the nucleus has an immature shape at E38 which is consistent with that seen prior to this period (Cucchiaro and Guillery, 1984), and no major changes in shape are seen to occur before this time.

\subsubsection{Evaluation of $L G N$ rotation.}

The concept of rotation when applied to three-dimensional structures can be difficult to interpret, especially when those structures are undergoing some form of structural change while rotating (as in the LGN). Here, rotation is defined from the anatomical borders of the nucleus, in a manner similar to that employed by Kalil (1978). To examine rotations that may occur in different planes, cross-sections were taken from each model, and the movement of the long axis of the nucleus relative to fixed anatomical landmarks was established. The long axis was determined by fitting a first degree elliptic Fourier function (Kuhl and Giardina, 1982) to the cross-section outline. Conceptually, this is equivalent to superimposing the best-fitting ellipse on the outline. The rotation of the nucleus in three-dimensions was evaluated by examining the movement of the first degree spherical harmonic function describing each model. Similarly, this is equivalent to superimposing the best-fitting ellipsoid to the threedimensional outline.

\subsubsection{Localised spatial shape change.}

One approach to examining transformations in shape would be to study the displacements of anatomical landmarks from one age to the next. However, the LGN has no identifiable landmarks at successive ages (except for the position of the optic nerve head within it at later ages), and hence a different approach is needed. 
By considering a series of vectors at equally spaced angles radiating from the origin of a nucleus, the points at which they break the surface will define a set of landmarks. If this is repeated for a nucleus at a different developmental stage, a second set of corresponding landmarks will be defined. It is then possible to calculate the displacement vectors between each corresponding landmark on the surface of both nuclei. The displacement vectors can be plotted as a vector field plot (Marsden and Tromba, 1996) allowing the spatial shape changes occurring to be visualised and measured. Figure 5.2 illustrates such a plot. The figure on the left (5.2a) shows the complete vector field, while the figure on the right $(5.2 \mathrm{~b})$ shows the same vector field with the first nucleus superimposed. The vectors illustrate the specific local spatial changes occurring in order to transform the first nucleus shown to the next developmental stage.
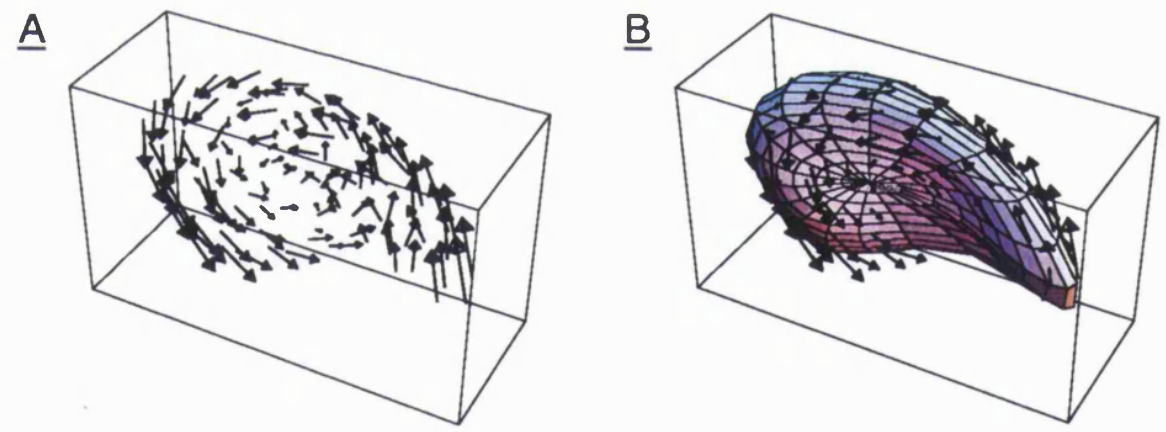

Figure 5.2 - Diagrams illustrating the concept of a vector field plot. The plots show the spatial changes occurring during the developmental period E38 to P0. Both plots show the same vector field, but $\underline{B}$ has the E38 nucleus superimposed. Models are normalised for their position in space only. The tail of each vector defines a landmark on the E38 nucleus, and the head of the vector, the corresponding landmark on the $\mathrm{P} 0$ nucleus. By considering a series of vectors at equally spaced angles, radiating from the origin of each nucleus, landmarks are defined as those points at which they break the surface. A cyclic motion is observed due to the fact that the models have not been normalised for rotation. It is shown in Section 5.3.2 that there is a rotation of the LGN between these ages, and this becomes apparent in the vector field plot shown in $\underline{A}$. 
This type of analysis simultaneously illustrates various localised features of shape change, and the interpretation of the results can be confounded. To aid interpretation, a course of analysis was devised as follows. First, the nuclei were split into rostral and caudal regions, approximately corresponding to those areas which will represent the monocular and binocular halves of the nucleus. Second, the calculated displacement vectors were split into two categories: those that showed nuclear expansion, and those that showed contraction. The evaluation of whether each vector is showing expansion or contraction is a non-trivial problem, and therefore an approximate approach has been adopted. As the results show (Section 5.3.6), a small number of vectors are classified as contracting when it is evident that they are actually illustrating expansion. Nevertheless, they provide an adequate representation of the specific pattern of local change. Finally, mean vector lengths (and SD) were calculated for expansion and contraction vectors in both rostral and caudal regions.

\subsection{Results.}

\subsubsection{The mean shape of the developing normal LGN.}

Surface models were successfully created for ferret LGNs at progressive stages of development. These allowed visual inspection of gross three-dimensional structural changes, as well as providing a quantitative description of those changes. Spherical harmonic coefficients of each model were used to calculate the mean LGN shape at successive developmental stages. Models representing the significant modes of variation (analogous to a S.D., Kelemen et al., 1997) could have been calculated, but have not been done so here due to the low number of animals in each sample. 
Figure 5.3 shows these mean shapes for animals ranging between E38 and P140, where orientation and scale is the same in all diagrams. A striking feature of these diagrams is the substantial expansion that occurs in the dorso-ventral axis, which is not immediately evident when looking in horizontally sectioned material. Measurements from the models show a 3.2 fold increase in this dimension between E38 and P140 (954 $\mu \mathrm{m}$ to $3121 \mu \mathrm{m})$, and this is by far the largest. The other axes (rostro-caudal and medio-lateral) increase by 1.35 and 2.4 fold. A lateral rotation of the rostral pole is also evident, beginning at around P5-P8, and complete at approximately P23-P36.

The models were used to calculate the volume of the LGN at each stage. Figure 5.4 shows LGN volume between E38 and P140. The data are fitted with a logistic curve. The first two postnatal weeks show very little volumetric change in the nucleus. This is in contrast to the three week period which follows, where there is a threefold change in volume. The adult volume of approximately $3.5 \mathrm{~mm}^{3}$ is reached by the $9^{\text {th }}$ postnatal week $(\approx \mathrm{P} 63)$. Comparisons with volume measurements made in the stereological study (Chapter 4) show good correspondence with the values obtained here.

This result is consistent with the notion that the models are representative of the anatomy. However, whether the models represent the true anatomical structure of the nucleus is difficult to ascertain, and while it is not pursued further here, it is addressed in Appendix C. For the remainder of the analysis, the models are therefore assumed to be faithful representations. 

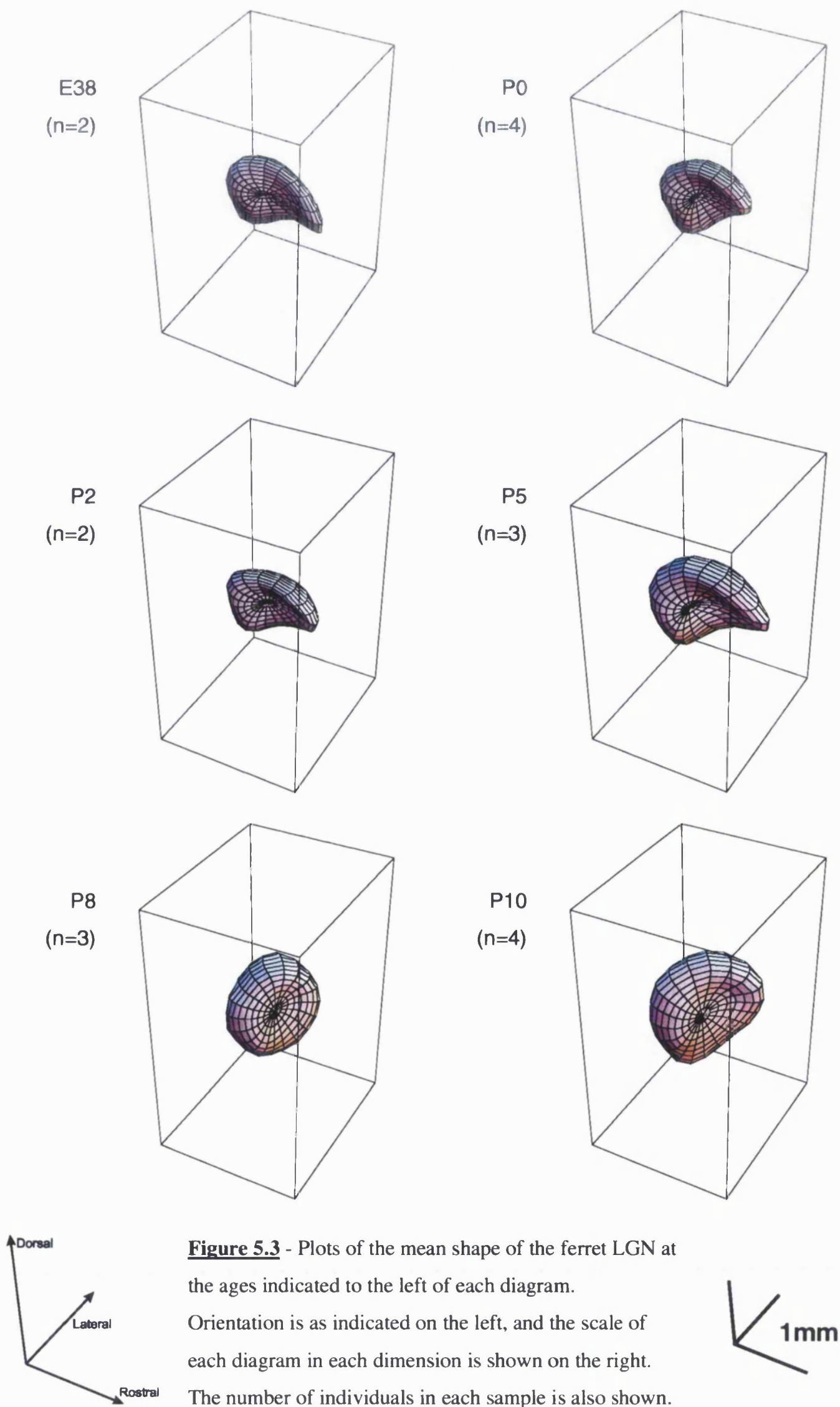

Figure 5.3 - Plots of the mean shape of the ferret LGN at the ages indicated to the left of each diagram.

Orientation is as indicated on the left, and the scale of each diagram in each dimension is shown on the right.

The number of individuals in each sample is also shown. 
P23

$(n=1)$

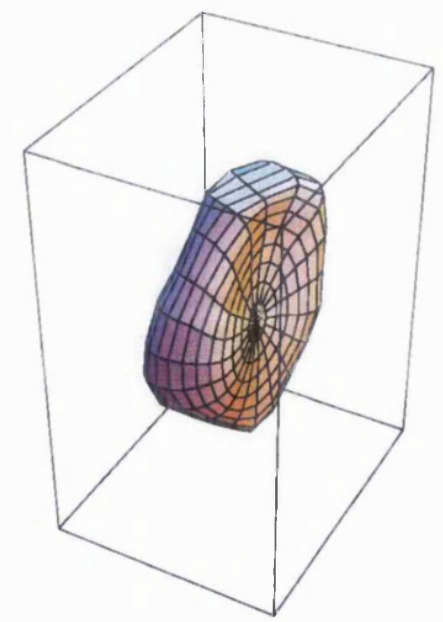

P47

$(n=2)$
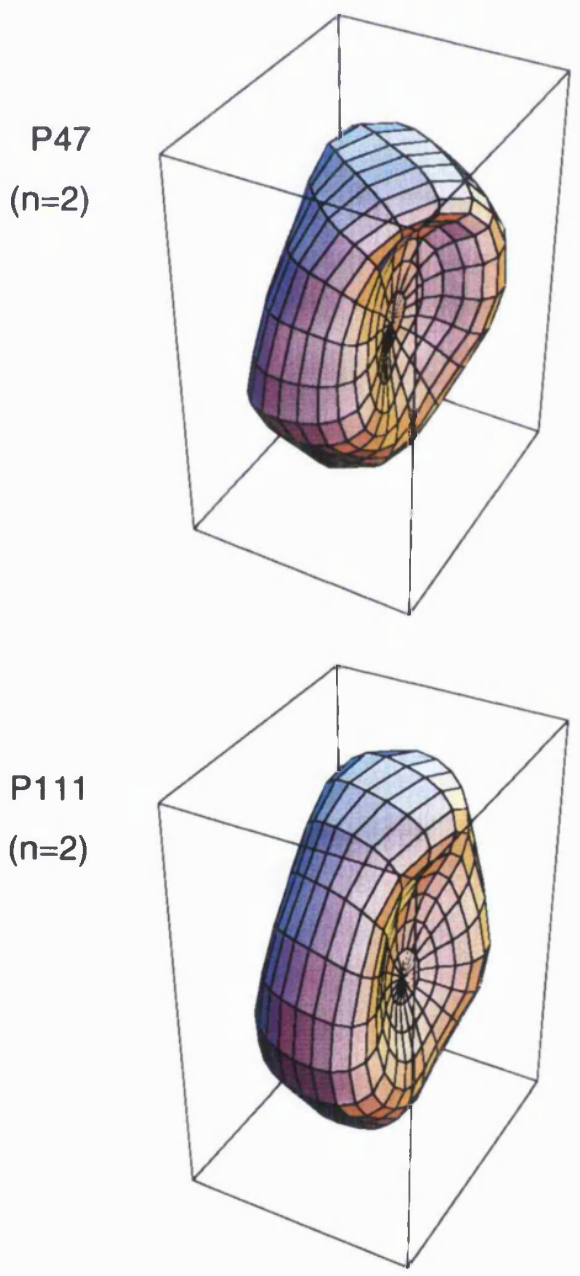

P36

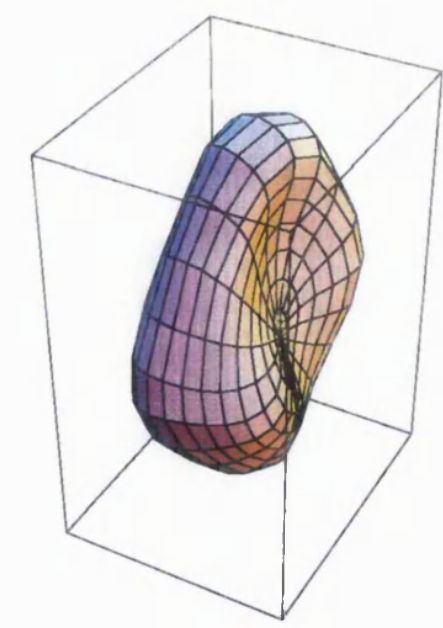

P83
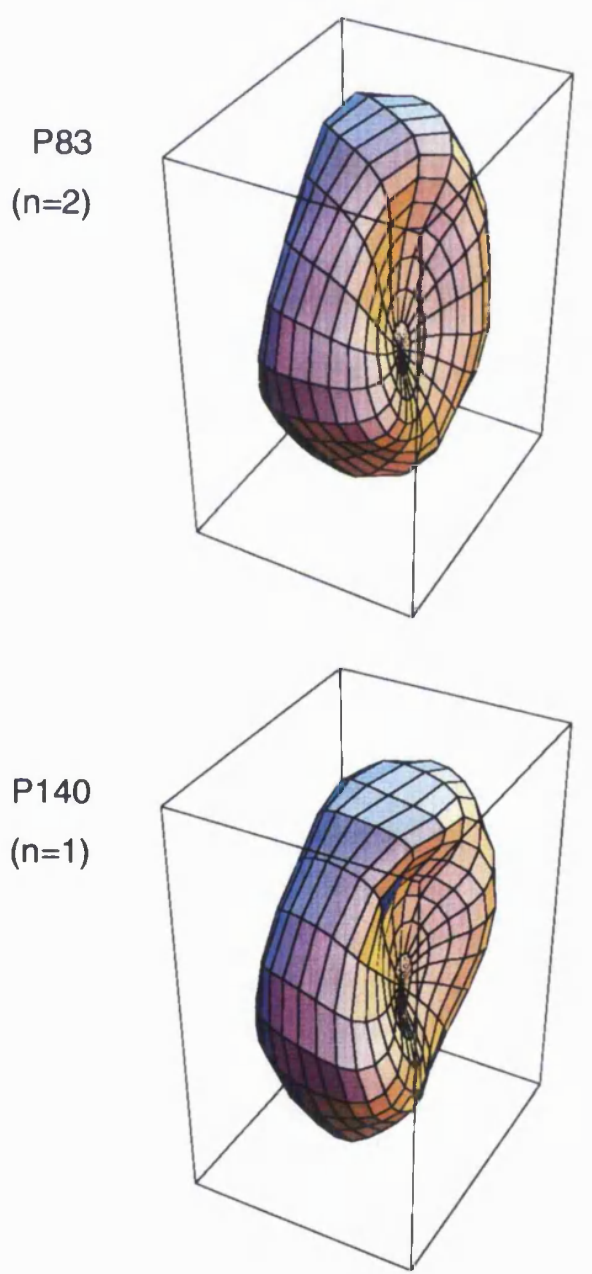

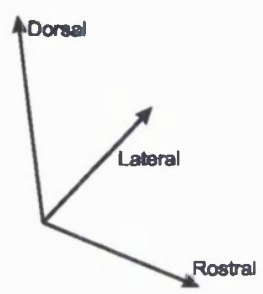

Figure $5.3\left(\right.$ cont $\left.^{\mathrm{d}}\right)$ - Plots of the mean shape of the ferret each diagram in each dimension is shown on the right.

The number of individuals in each sample is also shown. 


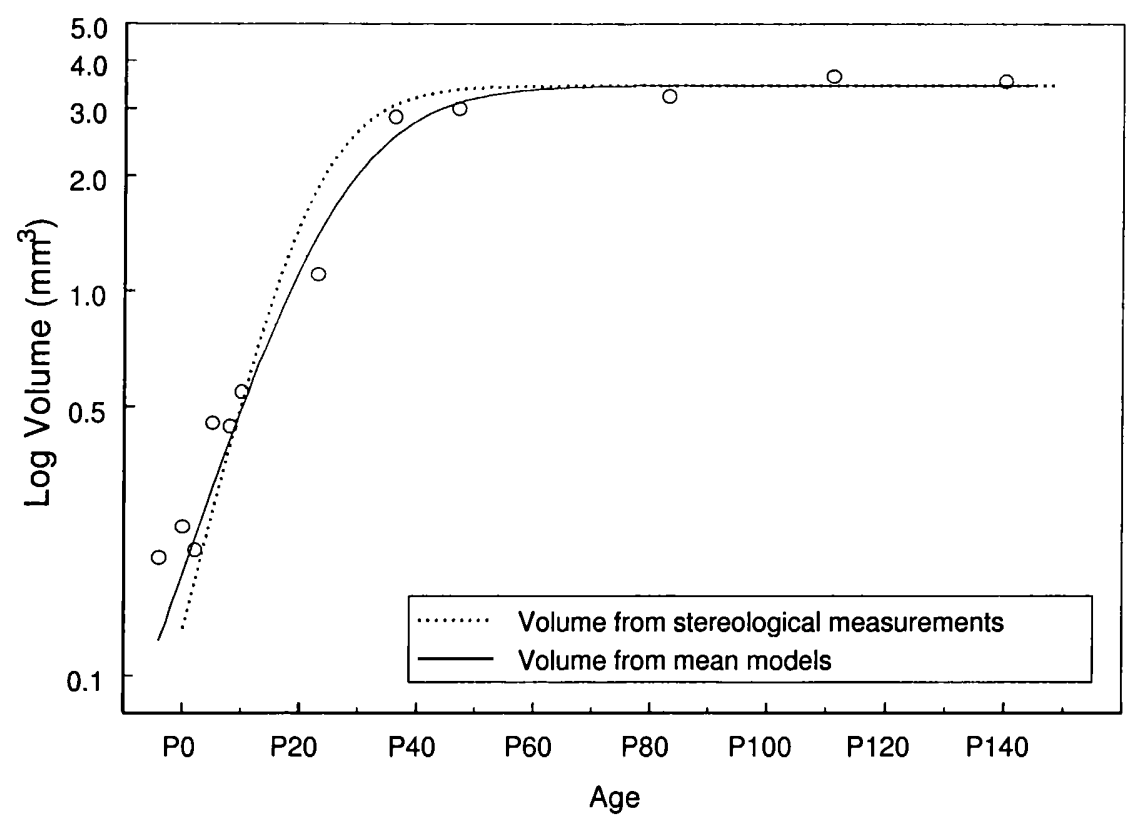

Figure 5.4 - Volume of the LGN $\left(\mathrm{mm}^{3}\right)$. Measurements are derived from the mean models shown in Figure 5.3. The y-axis is plotted on a logarithmic scale to highlight the data for the early developmental period, and the data are fitted with a logistic curve. Also illustrated (dashed line) is the growth curve for the volume of the nucleus as calculated during the stereological study (Chapter 4). A close correlation between the curves is apparent.

\subsubsection{Rotation of the LGN.}

LGN rotation was investigated in both two and three dimensions, using the mean models described earlier (Section 5.3.1). Examination of histological material in the horizontal plane shows a lateral rotation of the nucleus (see Figure 4.2). This has been measured using sections taken in the horizontal plane from mid-way between the dorsal and ventral poles of the models (Figure 5.5, filled markers, \& Figure 5.6). The long axis of the nucleus as determined from the first degree elliptic Fourier function shows a movement from the rostro-caudal plane into the medio-lateral plane. At E38, the nucleus is approximately parallel to the midline of the brain, and after approximately 3 weeks after birth, the nucleus is practically perpendicular to the midline, having displaced laterally by approximately $70^{\circ}$. This angular displacement is then maintained throughout maturity. 
If the LGN is viewed in the sagittal plane, and using the same criteria as above, a rotation of the nucleus is evident (Figure 5.7). However, the validity of this observation may be questioned, due to the rotation shown in the horizontal plane. The relationship between the long axis and what would be the monocular and binocular regions of the nucleus are preserved in horizontal sections. In sagittal sections, this relationship is preserved during the first postnatal week only. Due to the rotation in the horizontal plane, what would be the monocular and binocular regions of the nucleus are bisected by sagittal sectioning in the later stages of development. Therefore, an inconsistency in the relationship between the plane of section and the structure and organisation of the nucleus is introduced.

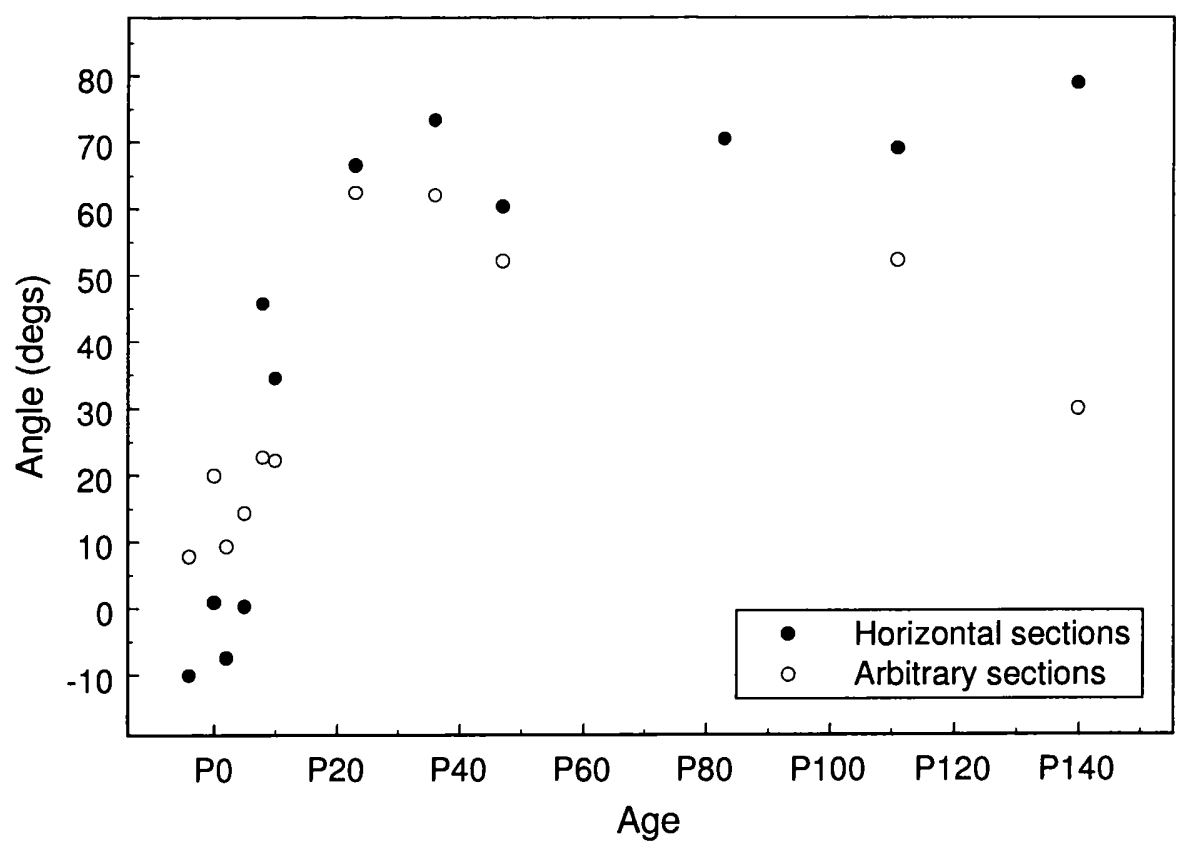

Figure 5.5 - Rotation of the LGN in two dimensional cross-sections as measured from the first degree elliptic Fourier function. Filled markers show the rotation of the nucleus in the horizontal plane, relative to the midline of the brain. Open markers represent the rotation of the LGN in an arbitrary plane, which takes account of the horizontal rotation, and is relative to the base of the thalamus. 
E38

$\Phi=-10.11^{\circ}$
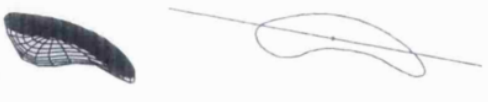

P23

$\Phi=66.55^{\circ}$

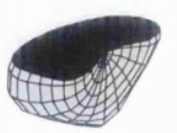

\section{P36}

P0

$\Phi=0.92^{\circ}$
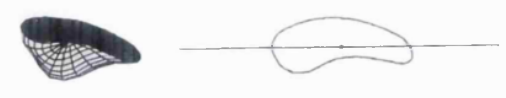

P2

$\Phi=-7.42^{\circ}$
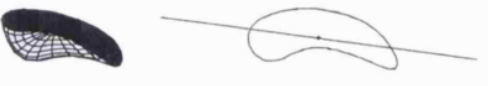

P5

$\Phi=0.33^{\circ}$

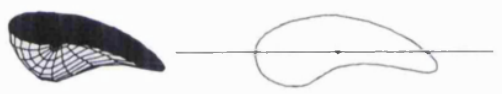

P 8

$\Phi=45.65^{\circ}$
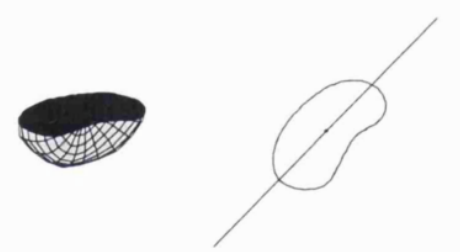

P111

$\Phi=69.01^{\circ}$

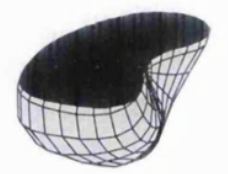

P 47

$\Phi=60.34^{\circ}$

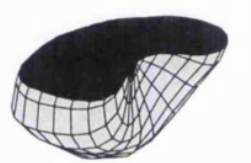

\section{P 83}

$\Phi=70.34^{\circ}$
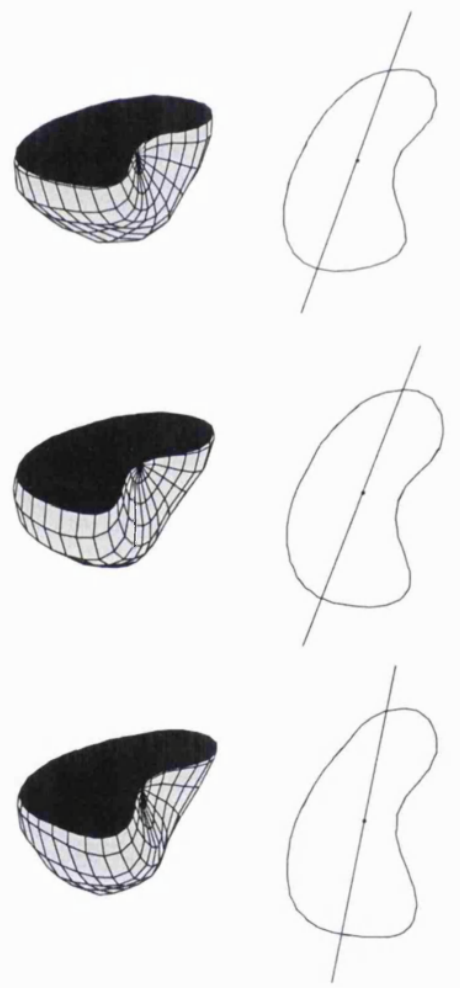

Figure 5.6 - Rotation of the LGN as seen in the horizontal plane, where a displacement of approximately $70-80^{\circ}$ can be seen. The three-dimensional plots illustrate the level in the dorso-ventral axis of the mean models from which the accompanying two-dimensional sections are taken. The line passing through each nucleus represents the position of the long axis of the best fitting ellipse (i.e. the first degree elliptic Fourier function). $\Phi$ is the angle between this axis and a reference axis parallel to the midline of the brain. 3D orientation is the same as in Figure $5.3 ; 2 \mathrm{D}$ orientation has lateral towards the top, and rostral towards the right. 


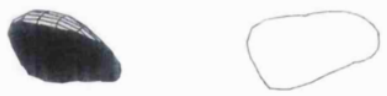

P23
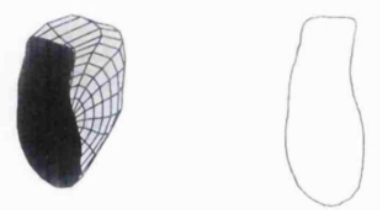

PO
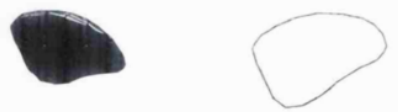

P36
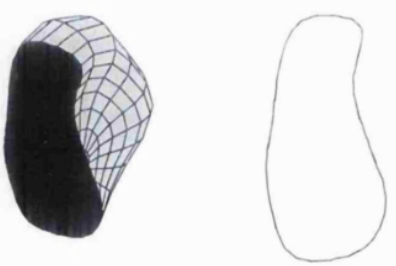

P2
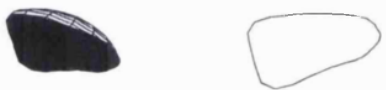

P47
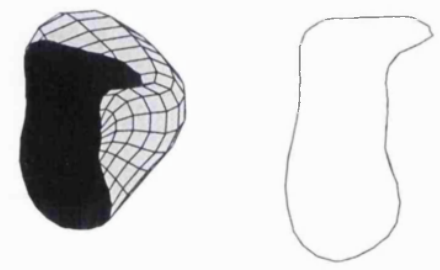

P5
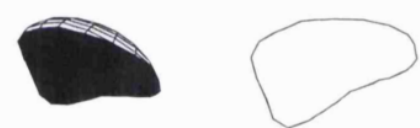

P83
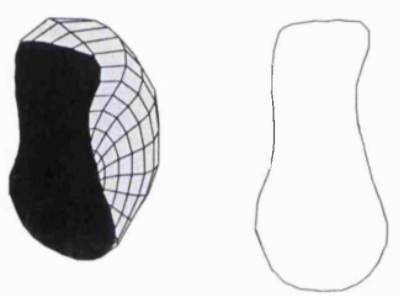

P8
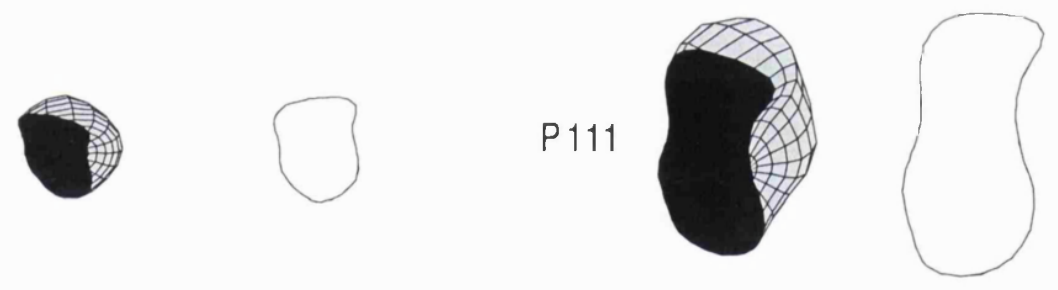

P111

P140
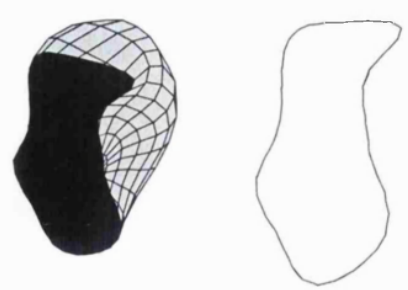

Figure 5.7 - LGN as seen in the sagittal plane. It is possible to measure a rotation of the nucleus in this plane, but the horizontal rotation introduces an inconsistency in the relationship between the plane of section and the structure of the nucleus. The three-dimensional plots illustrate the level in the medio-lateral axis of the mean models from which the accompanying two-dimensional sections are taken. 3D orientation is the same as for Figure 5.3; 2D orientation has dorsal towards the top, and rostral towards the right. 
To account for lateral LGN rotation, arbitrary cross-sections were taken in a plane perpendicular to the horizontal planes shown earlier (Figure 5.8). During early development, these cross-sections are equivalent to anatomically defined sagittal sections, but due to the horizontal rotation of the nucleus, gradually become coronal sections. A rotation of the nucleus can be measured in these sections (Figure 5.5, open markers), but not as clearly as for the horizontal sections. During early development (E38-P10), the angles of rotation are clustered around $10-25^{\circ}$, peaking between $\mathrm{P} 23$ and P36, and then falling during adulthood. In addition, at P83, the long axis of the elliptic Fourier function has flipped into a different quadrant $\left(-86^{\circ}\right)$, indicating that there may not be a consistency to the rotations observed in this arbitrary plane. Together, these results could be indicative of an expansion of the nucleus, particularly in the dorso-ventral axis as described earlier (Section 5.3.1), rather than a rotation.

Analysis of LGN rotation in three dimensions, again based on the long axis of the nucleus, but evaluated using the first degree spherical harmonic of the mean models confirms the above results (Figure 5.9). The small slits in the ellipsoids indicate the plane of the longest axis. The lateral displacement is immediately obvious, and illustrates the rotation observed in the horizontal sections. Additionally, there is a dorsal movement of the long axis, as observed in the arbitrary cross-sections.

These results indicate that the movement of the long axis of the LGN from a rostro-caudal direction to medio-lateral as shown in the horizontal plane is probably the only true rotation (or movement) of the nucleus. Angular changes measured in the arbitrary plane as described above may be considered an anomaly of the measuring scheme, and the greater expansion of the dorso-ventral axis rather than any other axis as shown in Section 5.3.1. contributes to this. 
E38

$\Phi=7.78^{\circ}$
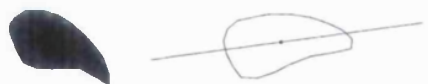

PO

$\Phi=19.85^{\circ}$
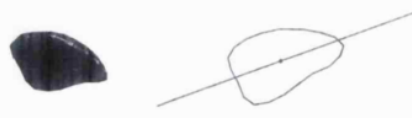

P2

$\Phi=9.29^{\circ}$
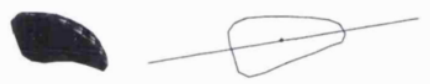

P5

$\Phi=14.27^{\circ}$
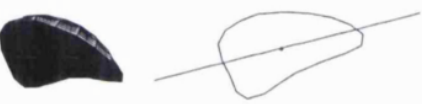

P8

$\Phi=22.56^{\circ}$
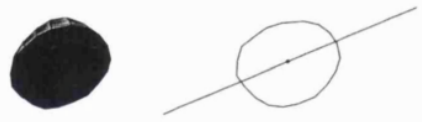

P 111

$\Phi=52.23^{\circ}$

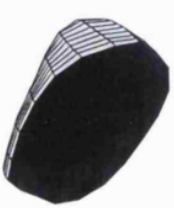

P47

$\Phi=52.11^{\circ}$
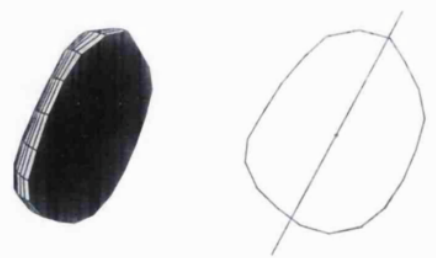

P83

$\Phi=-86.24^{\circ}$
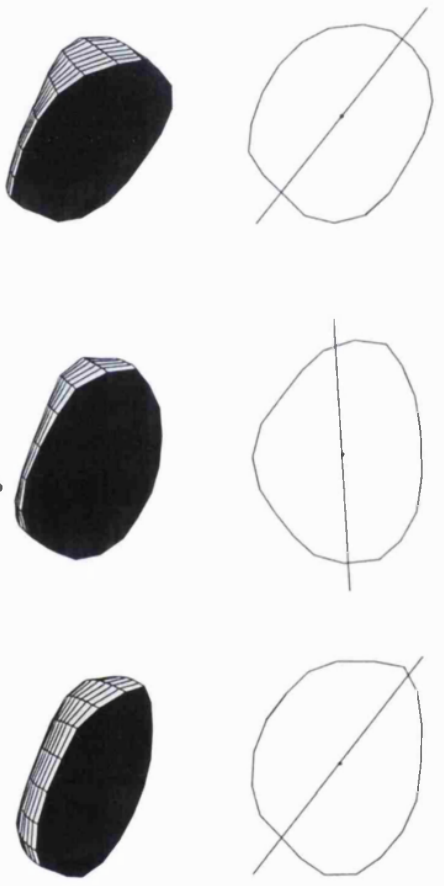

P140

$\Phi=29.92^{\circ}$
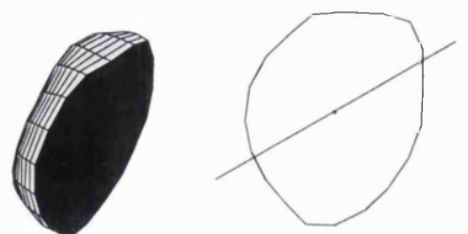

Figure 5.8 - Rotation of the LGN as determined from an arbitrary plane of section, which takes account of the horizontal rotation of the nucleus. The cross-sections are from a plane perpendicular to the horizontal plane, but parallel to the long axis of sections taken in the horizontal plane. The measurements do not show a clear course of angular change as was demonstrated for the horizontal sections, and may be indicative of an expansion of the nucleus as opposed to a rotation. 3D orientation is the same as in Figure 5.3; 2D orientation has dorsal towards the top in all cases, rostral is towards the right during early development, and due to the horizontal rotation, gradually becomes lateral. 

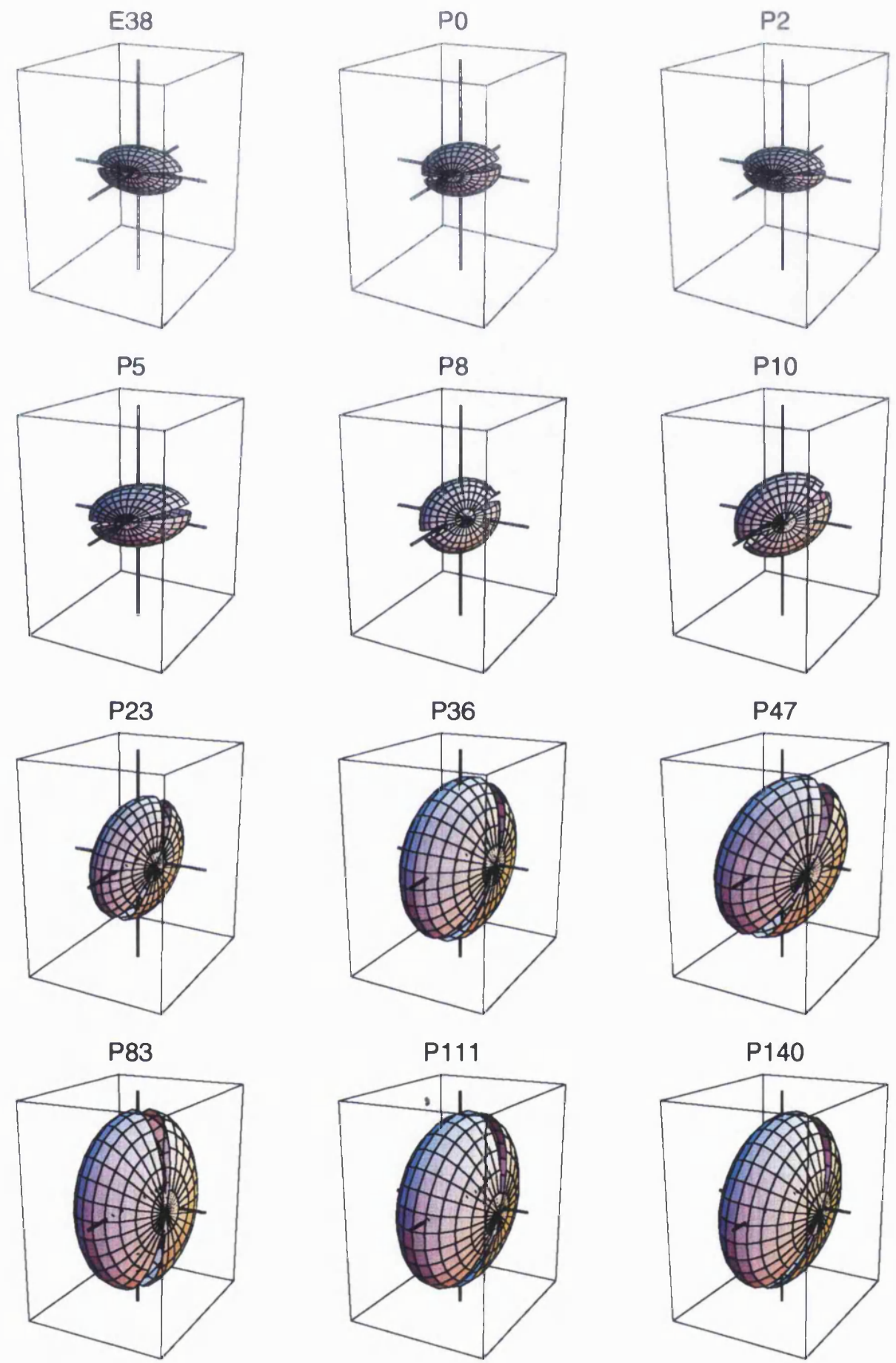

Figure 5.9 - Rotation of the LGN in three dimensions, based on the longest axis of the nucleus.

The diagrams show the first degree spherical harmonic functions of the mean models, essentially the best fitting ellipsoids. The small slits in the ellipsoids indicate the plane of the longest axis. The lateral rotation of the nucleus is immediately obvious, as is the rotation measured in the arbitrary plane. The greater expansion of the dorso-ventral axis rather than any other contributes to this perceived change. Orientation is the same as indicated in Figure 5.3. 


\subsubsection{A time course for the changes in LGN shape.}

Changes in the structure of the LGN are illustrated in Figure 5.3 above, although these are simply illustrative in nature. Here the temporal aspects of shape change in the LGN are described quantitatively, such that the progress of change with other events described in Chapter 4 can be compared in an attempt to determine any relationships between them. As outlined in Section 5.2.1, a measure of difference between shapes can be established by calculating the Euclidean distance between them. By making all the dissimilarity measurements relative to the youngest animal (E38), a measure of growth is provided. Figure 5.10a illustrates the dissimilarity between E38 mean LGN shape and the subsequent developmental ages modelled. Changes in LGN structure are complete by approximately P45. Note that the only object space normalisation applied to the data is for position in space (see Section 2.5.2.4), such that information concerning rotation and size are preserved. If size effects are removed (Figure 5.10b), the data indicate that no shape changes occur following P20.
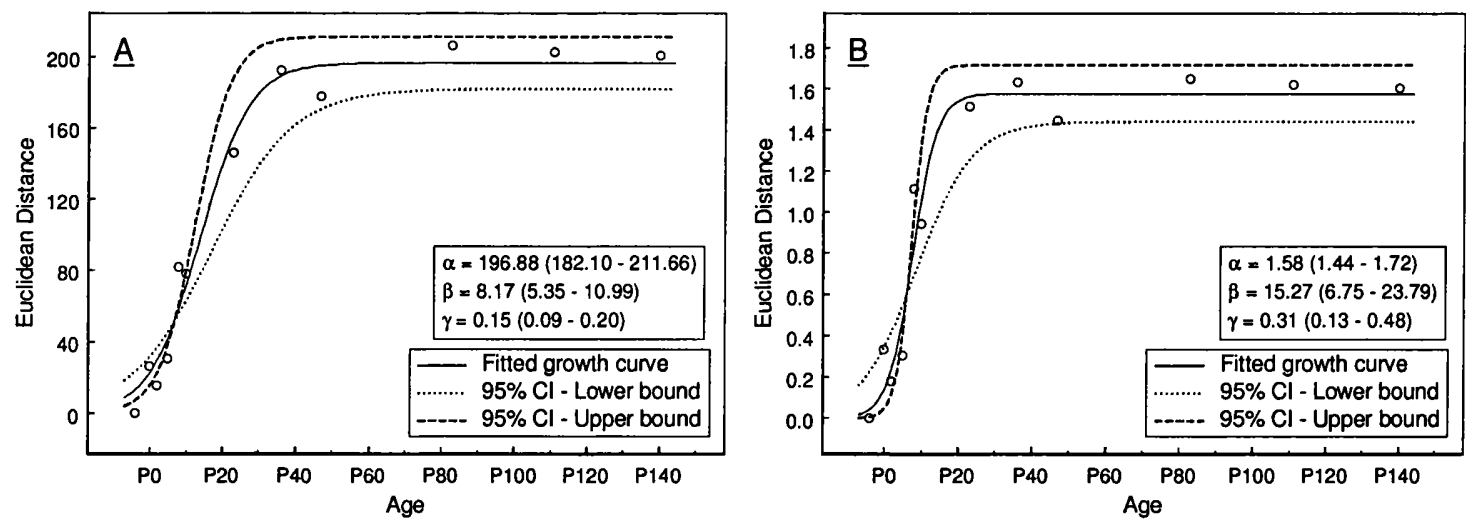

Figure 5.10 - Temporal changes in developing LGN shape. The graphs are derived by measuring the Euclidean distance (sum of squared differences between corresponding coefficients) between the E38 mean model and models at subsequent stages. $\underline{\text { A shows the }}$ change in LGN shape, and $\underline{B}$ shows the same change in shape, but with the effects of the increasing size of the nucleus removed. Growth curves have been fitted to the data, and the 95\% confidence intervals of the curve parameters are shown. 


\subsubsection{Temporal relationships between measured LGN parameters.}

Fitting data with growth curves allows for normalisation, and consequently permits comparisons to be made with other events fitted with similar curves (Section 2.6). Normalisation assigns the plateau value to $100 \%$, such that the curves describe the degree of maturity of the parameter being measured (i.e. $100 \%$ being the adult value). Figure 5.11 shows the maturation of LGN volume, cell density, cell size, and change in shape (the data from Figure 5.10a has been used for shape). During LGN development, the first feature to reach maturity is the shape of the nucleus at approximately $\mathrm{P} 45$. The cessation in shape change is closely followed approximately one week later (P50-P55) by the stabilisation of cell density. At approximately ten weeks after birth (P70), both LGN volume and cell size arrive at their adult values. Figure 5.12 shows that the rates at which these changes occur differ. The maximum rate of volume increase is at $\mathrm{P} 26$, whereas the maximum rate of cell growth occurs over two weeks earlier (P8), which incidentally, is when cell density is dropping at its highest rate.

Caution should be excercised in interpreting these curves since the parameters describing each curve $(\alpha, \beta, \gamma)$ incorporate a degree of error (see previous sections for confidence intervals of each parameter). Thus the time at which maturity is reached, and the time of maximum growth could be shifted temporally, while the rate of growth could also increase or decrease. This is particularly the case for the growth curve representing changes in LGN cell size, since the parameters of the curve have large 95\% confidence intervals due to the large standard deviations present in the original data (see Figure 4.6). 


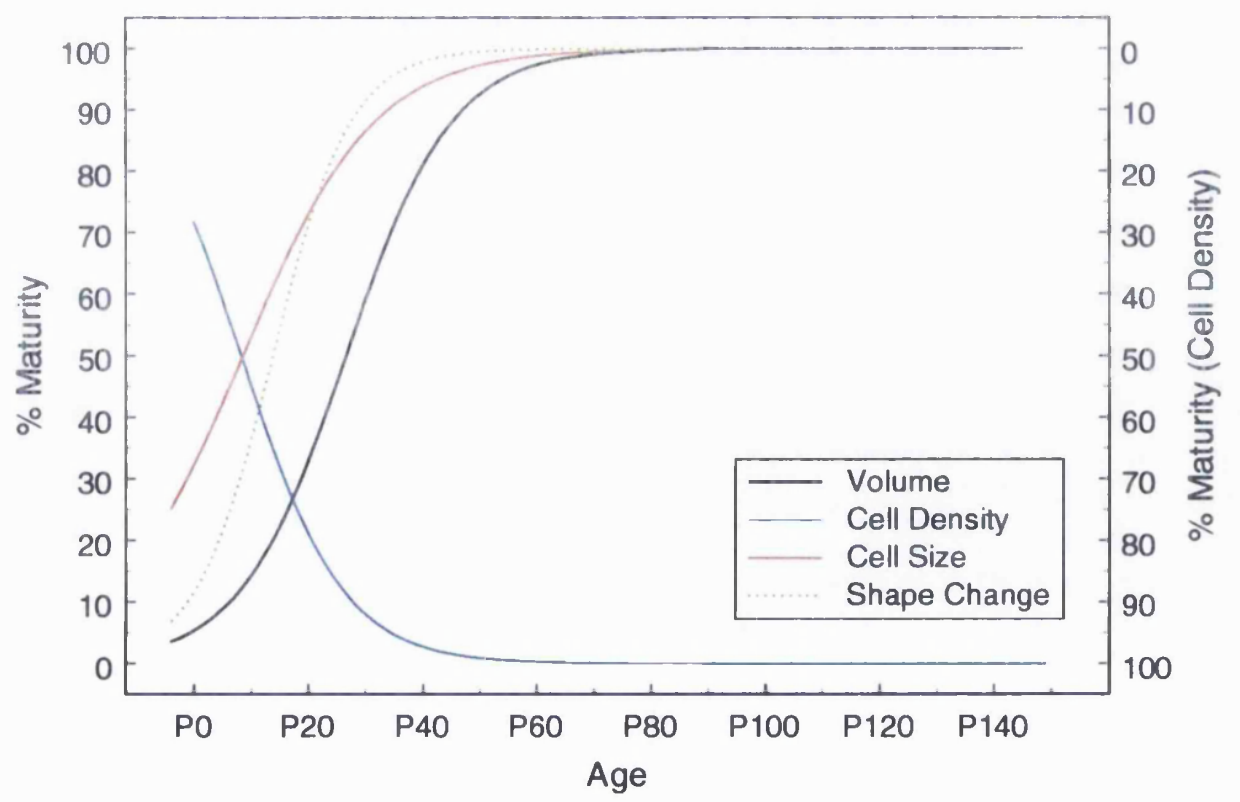

Figure 5.11 - Maturation of LGN volume, cell density and cell size within the nucleus, and change in its shape during development. The curves are derived by setting the plateau value to 100 (i.e. 100\% maturity being the adult state). Cell density is plotted against the right hand axis as it is a decaying process. LGN shape is mature by approximately P45. One week later cell density stabilises. Cell size and LGN volume reach their adult states at around P70.

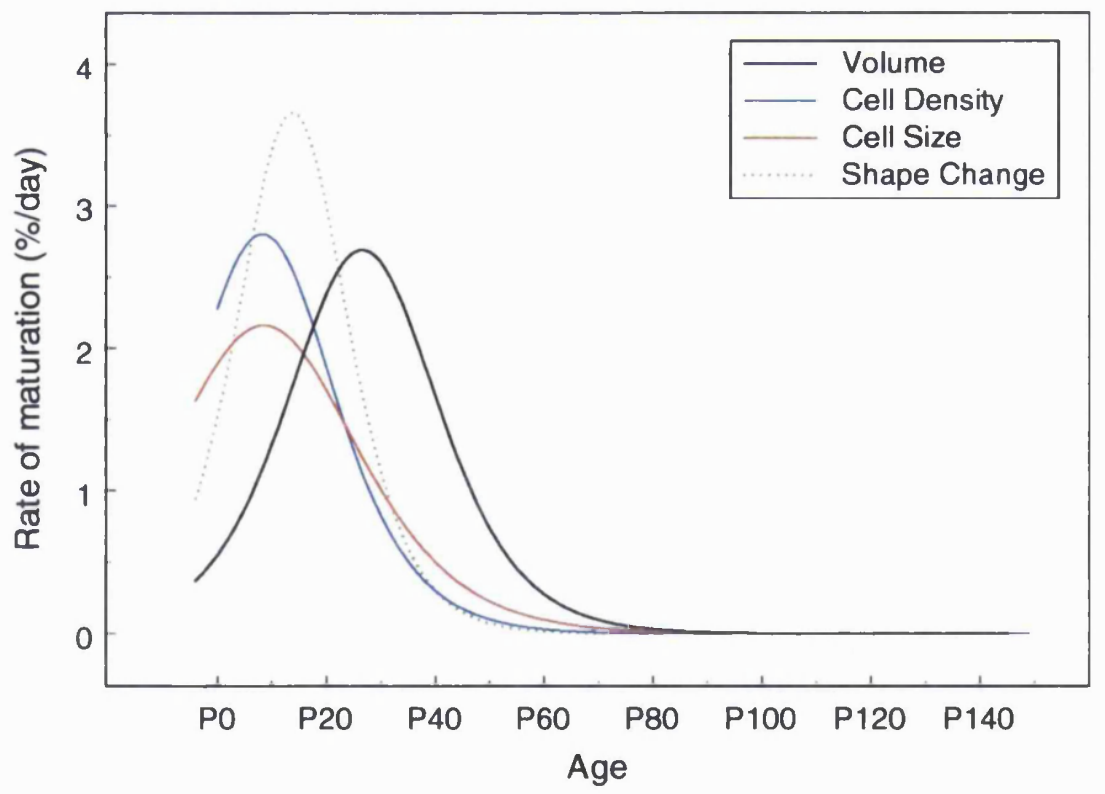

Figure 5.12 - The rate of maturation of LGN volume, cell density and cell size within the nucleus, and change in its shape during development. The curves are derived by taking the first derivative of those shown in Figure 5.11. The maximum rate of cell density decline coincides with the maximum rate of cell growth (at P8). Approximately one week later, shape is changing at its maximum rate, while volume peaks in growth rate at approximately P26. 


\subsubsection{What influences changes in shape?}

To explore the relationship between LGN shape and each of volume, cell size, and cell density, the time variable is removed from the curve fit equations (as shown in Figure 5.11), and shape is expressed as a function of one of the others (see Section 2.6). Figure 5.13 shows the resulting functions, which have additionally been normalised to express each LGN variable in terms of its degree of maturity.

Cell size and cell density within the nucleus produce similar effects on the maturation of LGN shape. When both these parameters have reached 50\% of their adult values, shape has only attained $25 \%$ maturity. Therefore, while large changes are occurring to cell size and density within the LGN, smaller changes are occurring to its shape. The changing volume of the LGN has an opposite relationship with shape; small changes in volume are accompanied by much greater changes to shape. Figure 5.13 shows that when LGN volume is $20 \%$ mature, the shape of the nucleus has attained $50 \%$ of its adult form. This is reversed when volume attains greater maturity, and shape change is almost complete, where large changes in volume are accompanied by very small changes in shape.

\subsubsection{Specific local LGN shape changes.}

Following the mapping of temporal change above, this section quantifies the main spatial transformations occurring during LGN development. The magnitude and direction (i.e. vectors) of specific local changes in shape are measured from the displacement of corresponding landmarks defined on the surface of nuclei at different developmental stages. Corresponding landmarks are defined on different nuclei by specifying the same angles in the parametric definition of each surface. 


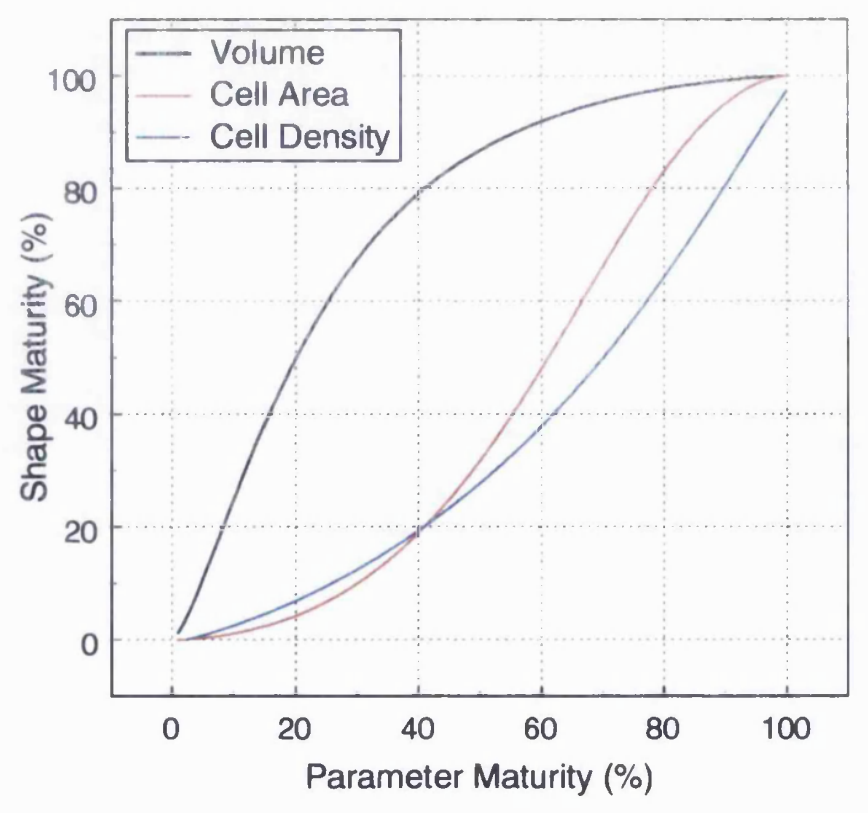

Figure 5.13 - Resulting plots when LGN shape change is expressed as a function of nuclear volume, cell size, or cell density. The functions have been normalised such that the variables are expressed in terms of maturity, where $100 \%$ represents the adult values. LGN cell size and cell density have similar relationships with the changes in nuclear shape. Large changes in each are accompanied by much smaller changes in shape. Volume has a different relationship with

changes in shape. While small changes in volume occur early in development, much larger changes in shape are observed.

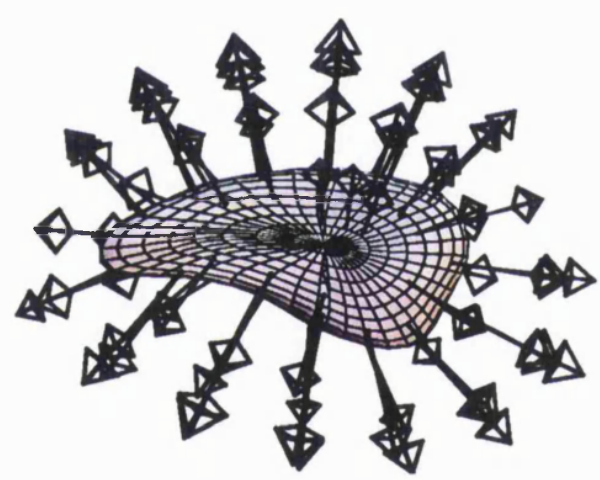

Figure 5.14 - Vectors showing specific local changes in shape between E38 and P140 nuclei. The nucleus shown is the mean E38 model, and the vectors show the main changes in shape required to transform it to the mean P140 shape. The medial surface of the nucleus is shown, rostral is to the left, and dorsal towards the top.

Figure 5.14 above shows the specific changes occurring to LGN shape between E38 and P140, and was produced according to the methods described in Section 5.2.3. The changes are extensive such that specific patterns of change cannot be discerned from this data. For this reason, specific local changes in shape are examined in smaller temporal windows.

The results of the analysis are shown in Figures 5.15 - 5.20. For the sake of clarity, three views of the LGN are provided: the first shows the medial surface, the second the posterior surface, and the third, the superior surface. In addition, 
differentiation is made between those vectors which show expansion of the nucleus, and those which show contraction. The previous section (Section 5.3.3) showed that shape change in the LGN was complete by approximately P50. Hence, all the models between P47 and P140 were averaged, and a generic adult model created. To simplify the analysis further, both P2 and P8 models were excluded. This was done since the former is similar to the P0 model, while the latter is similar to the P10 model (see Figures $5.3 \& 5.10$ a). Finally, and for clarity of illustration, only those vectors greater than the mean quantities were plotted. Since the measured SD's were large, this showed the major areas of expansion or contraction.

Spatial changes were examined in LGN models normalised for position and rotation. Position normalisation was applied since the models do not incorporate information regarding the LGN's position in the brain, allthough this may not be entirely appropriate (see discussion, Section 5.4). Rotation normalisation was necessary since Section 5.3.2 showed that there was a rotation of the nucleus. If this had not been applied, specific local changes in shape could be masked if they were smaller in magnitude than the rotation.

Between E38 and P0 (Figure 5.15), the calculated displacement vectors show that two major changes are evident: a contraction of the rostro-caudal axis, and an expansion of the dorso-ventral axis, both of a similar magnitude. This is completely reversed between P0 and P5 (Figure 5.16), where an expansion of the rostro-caudal axis occurs, forcing apart what will essentially become the monocular and binocular regions of the nucleus. No contractions of the nucleus could be measured between these ages. Spatial changes between P5 and P10 (Figure 5.17) are similar to those seen before birth (E38-P0, Figure 5.15): a contraction of the rostro-caudal axis, and an 
expansion of the dorso-ventral axis. These two modes of change complement each other in that when one axis shortens, the other lengthens. The measurements of the vector magnitudes confirm this: mean expansions and contractions are approximately the same $(\approx 90 \mu \mathrm{m}$ and $\approx 80 \mu \mathrm{m}$ respectively $)$.

From P10 onwards, the nucleus shows a general expansion in all directions (Figures $5.18 \& 5.19$ ). The magnitude of the changes are larger than those prior to P10, although the age ranges over which the observations were made are greater. Calculation of the mean magnitude of local changes by day (i.e. mean vector lengths for expansion and contraction over the entire nucleus) show them to be very similar (mean, 14.55 $\mu \mathrm{m}$; SD, $4.96 \mu \mathrm{m}$ ). By P36 (Figure 5.20) the nucleus has more or less attained its adult shape, the magnitude of the vector changes are small in comparison to the prior stages, and there is no distinct pattern to the changes. 

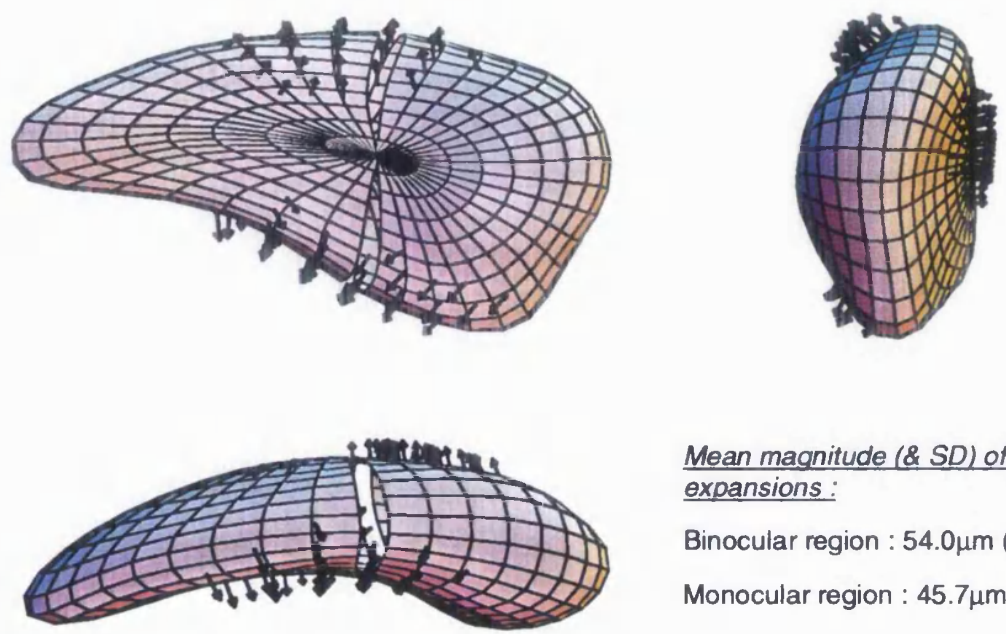

Mean magnitude ( $\& S D$ ) of the expansions:

Binocular region : $54.0 \mu \mathrm{m}(20.1)$

Monocular region : $45.7 \mu \mathrm{m}$ (19.1)
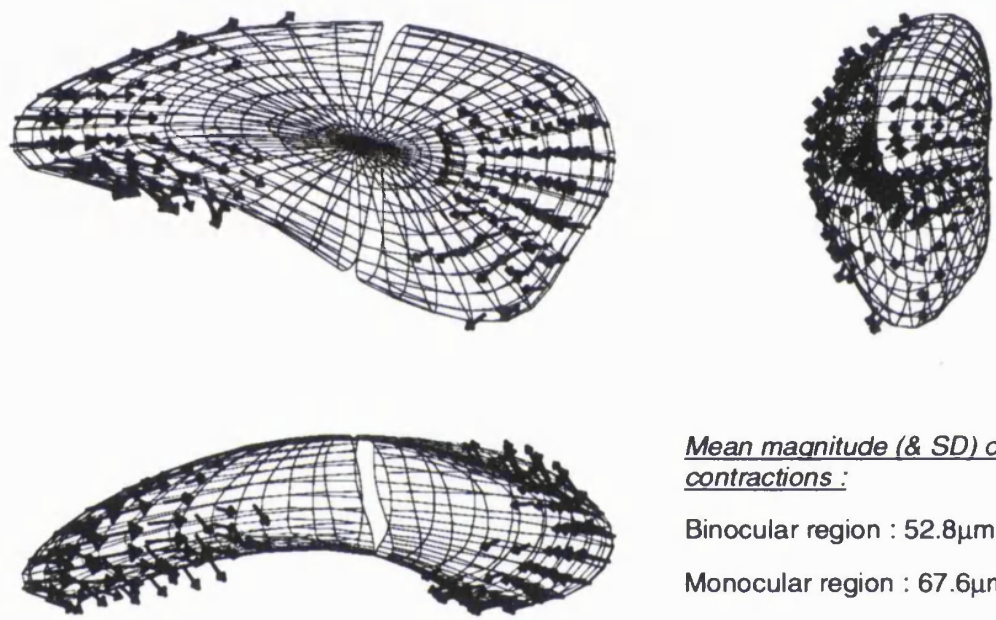

Mean maanitude (\& SD) of the contractions:

Binocular region : $52.8 \mu \mathrm{m}$ (17.6)

Monocular region : $67.6 \mu \mathrm{m}(26.4)$

\section{$500 \mu \mathrm{m}$}

Figure 5.15 - Vectors showing areas of greater expansion (solid figure) and contraction (wire figure) between E38 and P0 nuclei. The nucleus shown is the mean E38 model, and the vectors

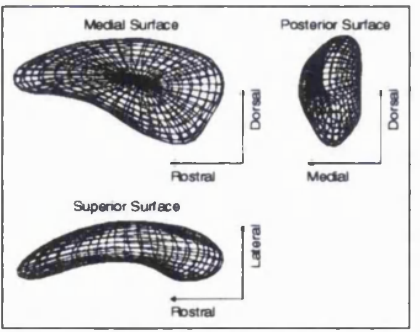

show the main changes in shape required to transform it to the mean P0 shape. For clarity, the vectors shown are those greater than the mean vector length calculated over the entire nucleus. The breaks in the surface of the nuclei indicate the arbitrary split into the presumptive binocular and monocular regions. Orientations are as indicated on the left, and scale as shown above. During this period, the major changes in shape are twofold: a contraction of the rostro-caudal axis, and an expansion of the dorso-ventral axis. A small lateral expansion is also evident. The contraction of the presumptive monocular portion of the nucleus is by far the largest change in shape. 

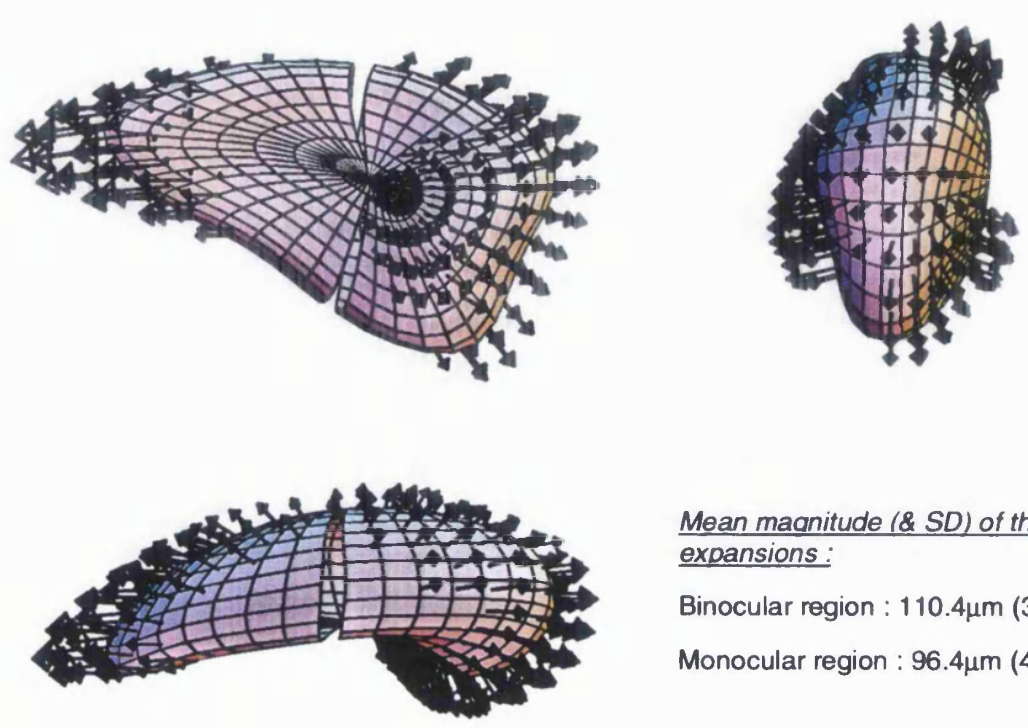

Mean magnitude (\& SD) of the expansions:

Binocular region : $110.4 \mu \mathrm{m}(32.6)$

Monocular region : $96.4 \mu \mathrm{m}(43.9)$

$500 \mu \mathrm{m}$

Figure 5.16 - Vectors showing areas of greater expansion between P0 and P5 nuclei. No contractions of the nucleus were evident at this stage. The nucleus shown is the mean P0 model, and the vectors show the main changes in shape required to

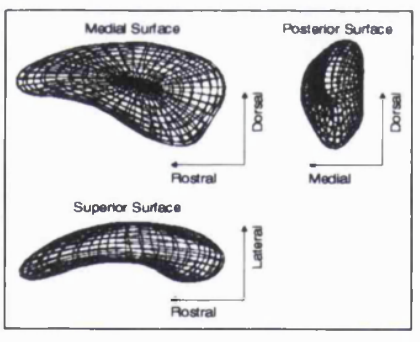
transform from $\mathrm{P} 0$ to the mean $\mathrm{P} 5$ shape. Other conventions are the same as in Figure 5.15. In contrast to the contraction of the rostro-caudal axis observed in the period E38-P0, this developmental period immediately following birth shows an expansion along the same axis. The monocular region is generally expanding in a rostral direction, while the binocular region is extending both caudally and medially. There is again some further growth of the lateral surface. 

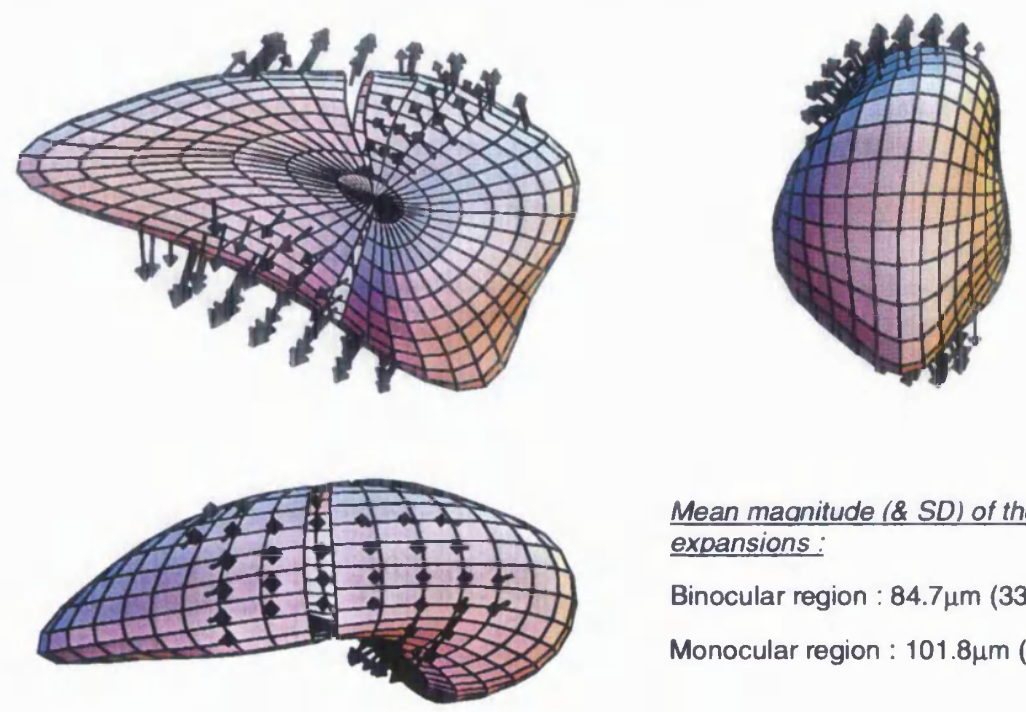

Mean maanitude (\& SD) of the expansions:

Binocular region : $84.7 \mu \mathrm{m}$ (33.7)

Monocular region : $101.8 \mu \mathrm{m}(47.5)$
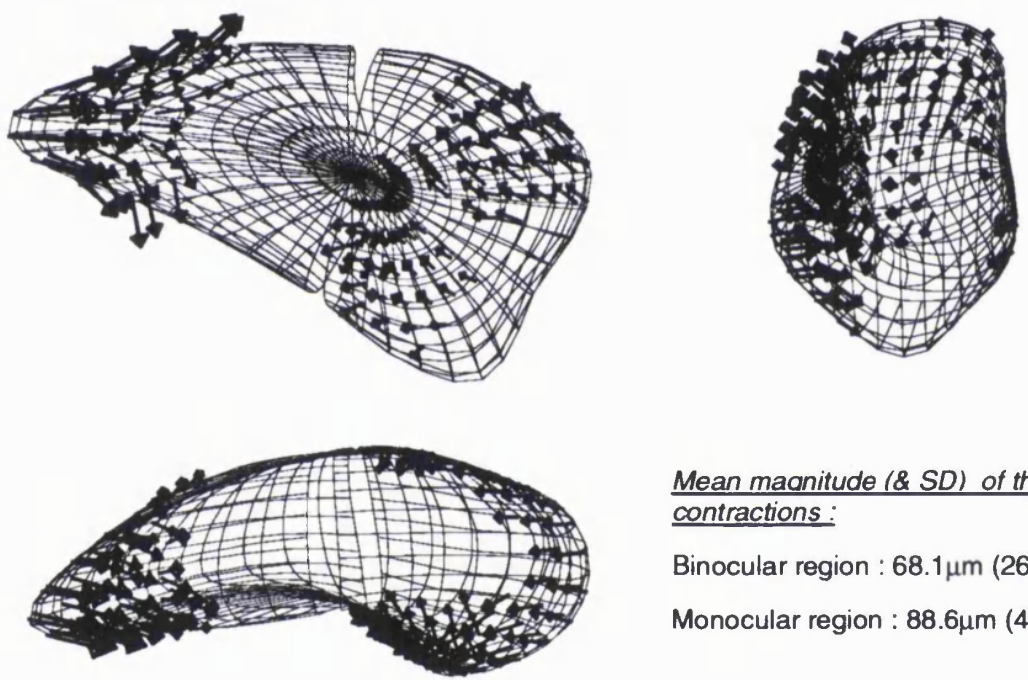

Mean maanitude (\& SD) of the contractions:

Binocular region : $68.1 \mu \mathrm{m}(26.6)$

Monocular region : $88.6 \mu \mathrm{m}(49.8)$

\section{$500 \mu \mathrm{m}$}

Figure 5.17 - Vectors showing areas of greater expansion (solid figure) and contraction (wire figure) between P5 and P10 nuclei. The nucleus shown is the mean P5 model, and the vectors

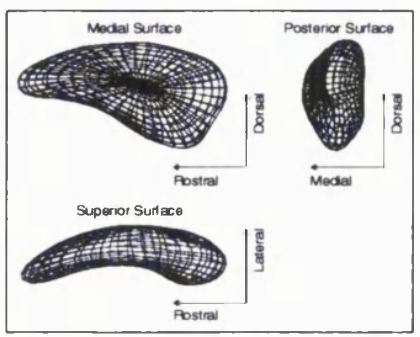
show the main changes in shape required to transform from P5 to the mean P10 shape. Other conventions are the same as in Figure 5.15. The mean magnitudes of the vectors indicate that the largest changes are the expansions of the superior and inferior surfaces. Simultaneously, there is a large contraction of the rostral (monocular) tip, as well as some smaller constrictions to the binocular regions of the nucleus. 

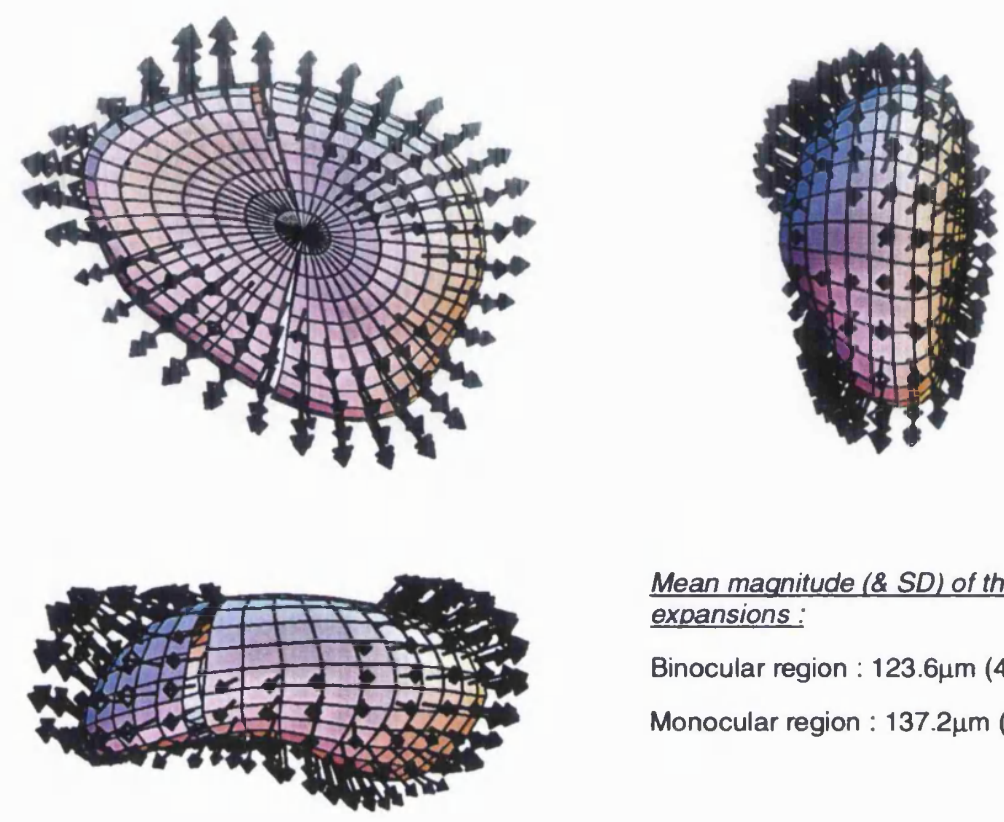

Mean magnitude (\& SD) of the expansions:

Binocular region : $123.6 \mu \mathrm{m}(47.4)$

Monocular region : $137.2 \mu \mathrm{m}(66.9)$

$500 \mu \mathrm{m}$

Figure 5.18 - Vectors showing areas of greater expansion between P10 and P23 nuclei. No

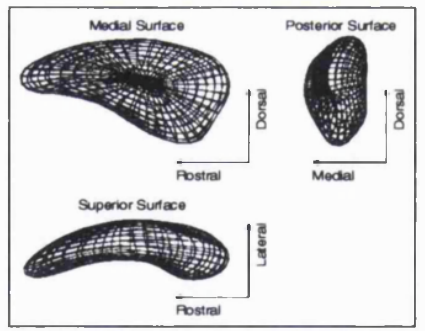

contractions of the nucleus were evident at this stage. The nucleus shown is the mean P10 model, and the vectors show the main changes in shape required to transform from P10 to the mean P23 shape. Other conventions are the same as in Figure 5.15. During this developmental period, the main changes in shape are a general expansion of the entire nucleus, bar the lateral and medial surfaces. Again, the mean magnitude of the vectors showing shape change indicate that the largest changes are occurring in the monocular region. 

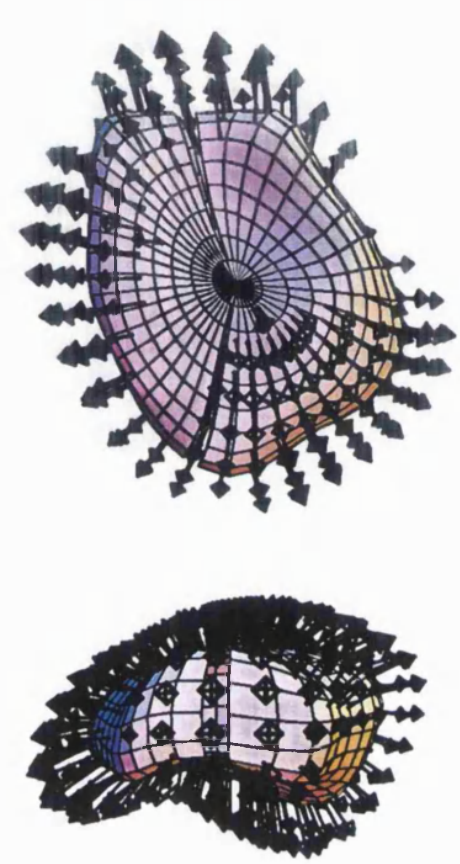

$500 \mu \mathrm{m}$

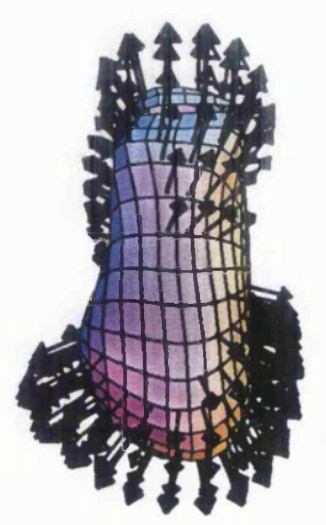

Mean magnitude (\& SD) of the expansions:

Binocular region : $246.8 \mu \mathrm{m}(67.6)$

Monocular region : $219.9 \mu \mathrm{m}(49.5)$

Figure 5.19 - Vectors showing areas of greater expansion between P23 and P36 nuclei. No contractions of the nucleus were evident at this stage. The nucleus shown is the mean P23

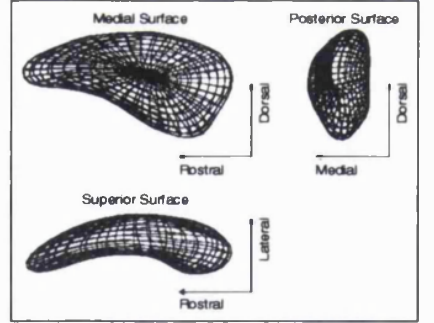

model, and the vectors show the main changes in shape required to transform from P23 to the mean P36 shape. Other conventions are the same as in Figure 5.15. Similar to the period P10-P23, this developmental period shows an overall expansion of the entire nucleus. There is an extensive expansion rostrally and dorsally, and likewise at the opposite end of the nucleus (caudally and ventrally). In addition, this binocular region of the nucleus shows a

large degree of expansion both laterally and medially. 

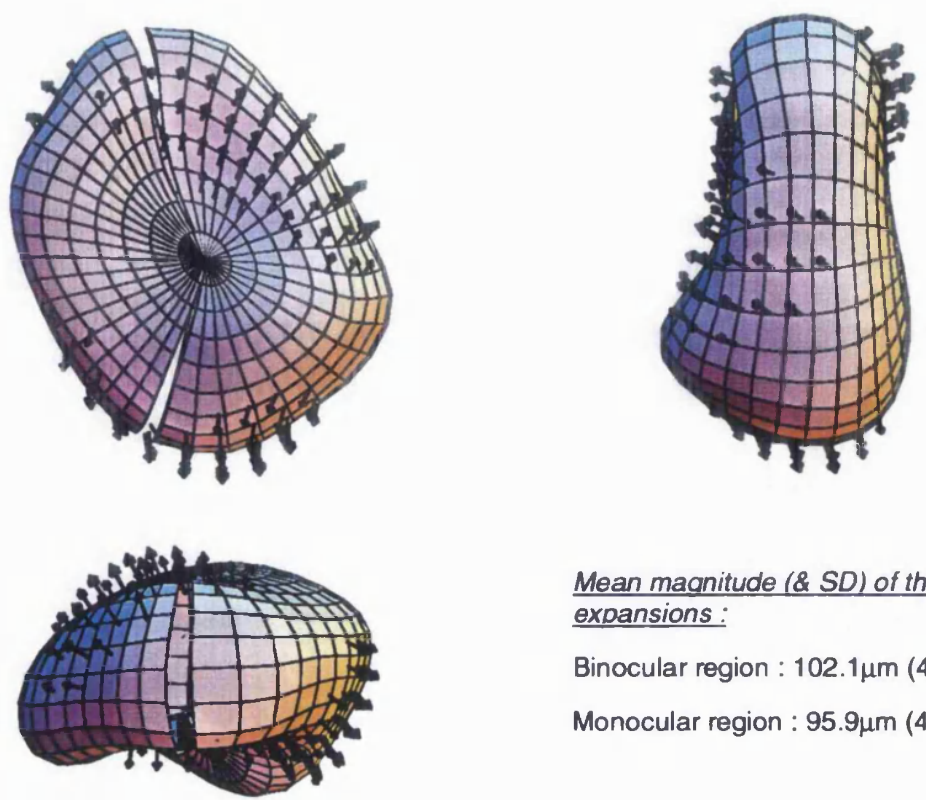

\section{Mean maanitude (\& SD) of the expansions:}

Binocular region : $102.1 \mu \mathrm{m}(44.9)$

Monocular region : $95.9 \mu \mathrm{m}$ (44.2)
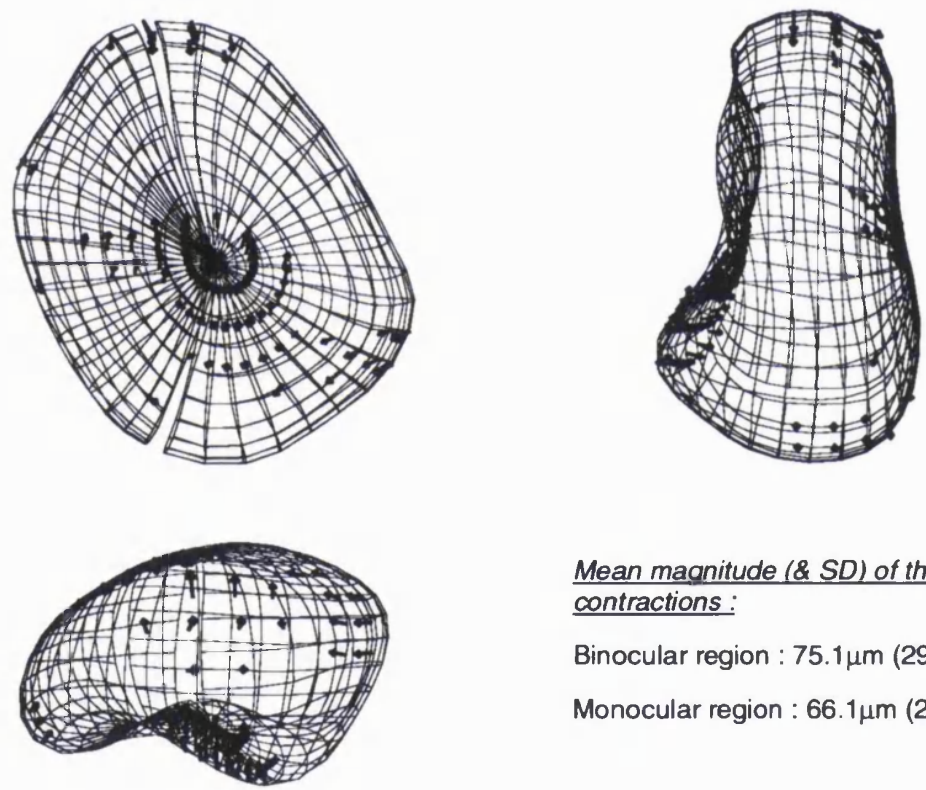

Mean maanitude (\& $S D)$ of the contractions:

Binocular region : $75.1 \mu \mathrm{m}$ (29.3)

Monocular region : $66.1 \mu \mathrm{m}(25.4)$

$500 \mu \mathrm{m}$

Figure 5.20 - Vectors showing areas of greater expansion (solid figure) and contraction (wire

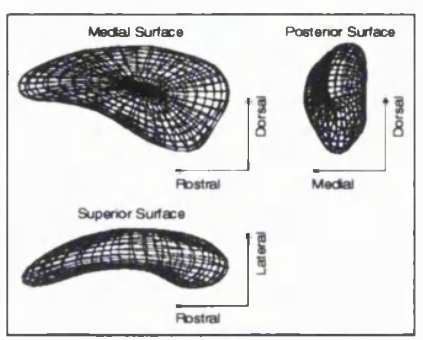

figure) between P36 and adult nuclei. The nucleus shown is the mean P36 model, and the vectors show the main changes in shape required to transform from P36 to the mean adult shape. Other conventions are the same as in Figure 5.15. The changes in shape during this period are almost negligible in comparison to the earlier period, and illustrate that the nucleus has virtually attained its adult shape. 


\subsection{Discussion.}

This chapter has presented data on three-dimensional shape changes in the developing ferret LGN, utilising recently developed structural modelling techniques. Models of the mean shape of the developing LGN have been produced, and these have facilitated an analysis of both the spatial and temporal aspects of growth. These aspects of growth have in turn been compared to other facets of the development of the LGN.

\subsubsection{Temporal correspondences during development.}

Among the various aspects of LGN development, the data provided here have shown that the shape of the nucleus reaches maturity before its volume, the density of cells within it, or their size. Linden et al (1981) reported qualitatively that the LGN attains "an essentially adult structure" by the $22^{\text {nd }}$ postnatal day. Here, quantitative analysis reveals that the nucleus is approximately $90 \%$ mature in terms of shape at this age, and the plots of the mean models illustrate that the major changes occurring after this stage are a general increase in nuclear size. The rate at which shape changes peaks at the end of the second postnatal week, coincident with the time at which the cytoarchitectonic borders between layers A and A1 become clearly defined, and when retinogeniculate fibres have almost completed segregating (Linden et al., 1981; Cucchiaro and Guillery, 1984).

Shape descriptors as employed here contain information concerning not only the form of the LGN, but also its size and position. In analysing shape changes, the substantial increase in the size of the nucleus could influence the interpretation of the results. Here, it is shown that when the effects of size are removed from the models of LGN shape, no further changes in the structure of the nucleus occur after P20. This 
implies that the LGN changes in shape during the exact period when cytologically distinct laminae are formed, and projections from each eye segregate into their respective regions. When the process is complete, all that is observed is a change in the size of the nucleus.

Since LGN shape is the first parameter to reach maturity, it may be the case that it is not entirely independent of other factors (volume, cell area, cell density). The degree to which shape change is related to these factors has been explored by expressing shape as a function of these other variables. Cell area and cell density have similar relationships with shape change. At a time when both have reached half their adult values, the shape of the nucleus is only $25 \%$ mature. Volume of the nucleus has the opposite relationship and contributes much to shape maturity very early in development.

The combination of these results, and those provided in Chapter 4, lead to a conclusion that the cellular constituents of the LGN contribute little to the changes in its shape. The dendritic fields of LGN cells, and arbors of retinogeniculate and corticogeniculate axons may play a larger role in the formation of LGN shape, particularly since they probably contribute a substantial amount to LGN volume. Additionally, while this study has not examined the growth dynamics of the environment surrounding the nucleus, it is envisaged that this must also play a role in generating the characteristic shape of the LGN. Regions which may be of particular interest include the optic tract (the LGN is bound on its lateral margin by it), and various thalamic nuclei adjacent to the LGN (e.g. ventral LGN, medial geniculate nucleus, and the lateral posterior thalamic nucleus). 


\subsubsection{Specific shape changes during development.}

In the same way that size can affect the analysis of shape change, rotation can also be influential, and mislead the interpretation of results. Other studies have reported a rotation of the LGN in different species (primate: Rakic, 1977; cat: Kalil, 1978; Shatz, 1983). Here, it is shown that between $\mathrm{E} 38$ and P23, the LGN is gradually rotated by approximately $70^{\circ}$ from a rostro-caudal to a medio-lateral axis, and does not rotate significantly in any other direction. If this period is considered relative to conception, then the same displacement is seen to occur in the cat over the same time (E32 - E60, Shatz, 1983). Whether the rotation of the nucleus is simply a result of the growth of adjacent thalamic regions or serves a purpose that has yet to be identified is not known.

Analysis of spatial changes in LGN shape were carried out with models which had been normalised for rotation and spatial location. The former normalisation was applied due to the rotational displacement described, and the latter since the models did not incorporate information concerning the LGN's position within the brain. Whether this latter normalisation produces results which do not reflect the true nature of the changes is not known. For example, models compared by being superimposed on their centroids (as is the case here) may show an expansion in both rostral and caudal directions, whereas if they were superimposed on the caudal pole, they would show an increased rostral expansion.

In spite of the above, the results show that the nature of the spatial changes are consistent with observations made in other studies. Jeffery $(1985 ; 1989 ; 1990)$ has shown that before RGC axons segregate into distinct laminae, the terminals from each eye are arranged as orderly retinotopic maps. The studies demonstrated that these 
retinotopic maps have to slide relative to each other to produce the pattern of binocular registration seen in the adult. Jeffery (1990) proposed that the nasotemporal division at the caudal pole of the nucleus could act as the first point of registration. When this is established, the maps shift in this axis (rostro-caudal) to bring about the adult pattern.

The results shown here for the changes occurring between P0 and P5 illustrate that the changing shape of the LGN might contribute to this process of shifting maps. Between these ages, there is a large expansion in both the rostral and caudal poles of the nucleus which could underpin ocular segregation. But, the changes occurring between P5 and P10 are confounding: first, the main areas of nuclear expansion are at the dorsal and ventral borders, although the directions of the changes are in line with the idea of the maps being forced apart (i.e. they are in opposite directions). Second, there is a contraction in the same axis as the expansion which occurred during P0-P5, although of a smaller magnitude. It is difficult to ascertain how these changes could be related to the changes that would be necessary to bring the maps into register with each other as described by Jeffery $(1989 ; 1990)$. A number of issues contribute to this problem: the work of Jeffery $(1989 ; 1990)$ was carried out in two-dimensional horizontal sections, whereas the results presented here are in a three-dimensional framework. Additionally, the LGN models presented here are compared by centering on their geometric centres of gravity. This may not provide true descriptions of change. It is suggested that any future work incorporate the precise co-ordinates of the LGN within the brain, such that the resulting models represent the true anatomical position of the nucleus. 
Following the main period of laminar formation and fibre reorganisation within the nucleus, the later stages of development analysed (i.e. >P10) show the LGN expanding isotropically, and no particular direction of growth is observed. This type of growth is possible if all the constituent cells of the LGN and their respective processes are oriented relatively randomly and grow at similar rates (Van Essen, 1997). It also explains why this and other subcortical structures achieve a compact, rounded shape. Additionally, this relatively general pattern of growth is in contrast to the specific modes of growth occurring between ages prior to P10. While the majority of fibre reorganisation and laminar formation is complete by this age, the entire process is not wholly complete until the end of the second postnatal week (Linden et al., 1981). From this point of view, it would have been of interest to have an additional LGN model from this developmental period, to ascertain whether there were specific patterns of growth, or simply isotropic growth. 


\section{Models of the developing ipsilateral \& contralateral projection areas in the LGN.}

\subsection{Introduction.}

The measurements made in Chapter 3 of terminal field sizes from each eye within the LGN, as well as the volume of the nucleus, indicate that differential nuclear growth could contribute to ocular segregation. Here, the role of LGN shape change in the development of ocular segregation is investigated. If changes in LGN shape reflect similar changes in the shape of the terminal field region, then there may be a relationship between them. However, it has been shown here (Chapter 3) and elsewhere (Linden et al., 1981; Cucchiaro and Guillery, 1984), that the terminal field of the contralateral projection is different, and more substantial than the ipsilateral projection. At birth, both terminal fields are of a similar size, and during development, the terminal field of the ipsilateral projection retreats to the posterior aspect of the nucleus, while the contralateral projection withdraws from a similar region, which is subsequently occupied by the ipsilateral projection.

If LGN shape change is to play a role in ocular segregation, then it can only act as a mechanism for one of these processes - the retreat of the ipsilateral projection, or the 
withdrawal of the contralateral projection. However, if one facilitates the other (i.e. the retreat of the ipsilateral projection causes the withdrawal of the contralateral projection, or vice versa), then a means by which the changing shape of the nucleus could influence ocular segregation is evident. This concept is explored here. The relationships between the shape of the nucleus and both the retracting ipsilateral projection and the emerging space of the contralateral projection are analysed.

Two approaches utilising shape descriptors described earlier (Chapters $2 \& 5$ ) are adopted to investigate the potential relationship between the changing LGN shape and terminal field regions. The first establishes the transformation rules which describe changes in LGN models between two time points ( $t_{1}$ and $t_{2}$ say). These transformations are subsequently applied to models of terminal field regions at $t_{l}$, which produce hypothetical models of terminal field regions at $t_{2}$. Comparison of these models with their original counterparts indicates how well LGN shape changes describe transformations in the shape of terminal field regions.

The second approach analyses the specific spatial changes occurring to both structures between two time points. A vector analysis similar to that described in Section 5.2.3. is used, and comparisons are made between corresponding vectors describing LGN shape changes, and those which describe changes to terminal fields.

\subsection{Methods.}

\subsubsection{Construction of models.}

Drawings of the LGN and terminal field regions within it derived in Chapter 3 were used to create three-dimensional models of the nucleus and their respective terminal field regions (see also Section 2.5.2). In the LGN ipsilateral to the HRP 
injected eye, terminal field models represent the region of the nucleus containing label. Contralateral to the injected eye, terminal field models represent the region of the nucleus containing no label ${ }^{1}$. These models were employed to calculate mean models of the developing structures, which were subsequently used in the analysis described below. Table 6.1 summarises the number of models generated at each age.

\begin{tabular}{lcccc}
\hline \multicolumn{1}{c}{ Age } & P0 & P5 & P8 & P12 \\
\hline LGN Models (Ipsi \& Contra) & 3 & 3 & 3 & 3 \\
Terminal Field Models (Ipsi \& Contra) & - & 3 & 3 & 3 \\
\hline
\end{tabular}

Table 6.1 - Numbers of three-dimensional models created for the shape of the LGN and the terminal field region at the ages indicated, both ipsilateral and contralateral to the injected eye. No terminal field models were created for P0 since label fills the nucleus on both sides of the brain at this age. Models of the nuclei therefore sufficed as models of the terminal field region.

\subsubsection{Calculation of transformation matrices describing global shape change.}

As two structures are described by two matrices, it is possible to calculate a third matrix which describes the transformation between them. For example, given the matrices $A$ and $B$, the transformation matrix $T$ would describe the actions necessary to produce $\mathrm{B}$ from $\mathrm{A}$, such that :

$$
A . T=B
$$

This assumes a linear transformation law, and the solution to this equation may be calculated as :

$$
T=A^{-1} \cdot B
$$

\footnotetext{
${ }^{1}$ Throughout the remainder of this chapter, references to the contralateral terminal field refers to the region of the LGN contralateral to the injected eye which is void of label, i.e. the emerging space which the terminal field of the contralateral projection creates as it withdraws from this region.
} 
The matrix $T$ was calculated between a number of age groups for models of nuclei both ipsilateral and contralateral to the injected eye. Table 6.2 shows the transformation matrices calculated during the course of analysis, and in doing so, introduces some of the nomenclature used.

\begin{tabular}{llll}
\hline & \multicolumn{3}{c}{ Age Range } \\
\cline { 2 - 4 } & $\mathrm{P} 0 \Rightarrow \mathrm{P} 5$ & $\mathrm{P} 5 \Rightarrow \mathrm{P} 8$ & $\mathrm{P} 8 \Rightarrow \mathrm{P} 12$ \\
\hline Ipsilateral LGN & $T_{i n}^{P 0 \rightarrow P 5}$ & $T_{i n}^{P 5 \rightarrow P 8}$ & $T_{i n}^{P 8 \rightarrow P 12}$ \\
Contralateral LGN & $T_{c n}^{P 0 \rightarrow P 5}$ & $T_{c n}^{P 5 \rightarrow P 8}$ & $T_{c n}^{P 8 \rightarrow P 12}$ \\
\hline
\end{tabular}

Table 6.2 - Listing of the transformation matrices calculated for changes between LGN models spanning the ages indicated. The subscripts always reference ipsilateral (in) or contralateral nuclei $(c n)$. Two other subscripts which will be referenced in the following pages are $i p$ and $c p$, which refer to ipsilateral and contralateral projection areas (i.e. terminal field regions). Superscripts indicate the age range over which the transformations are calculated. As an example, $T_{i n}^{P O \rightarrow P S}$ represents the transformation matrix of the ipsilateral LGN models between $\mathrm{P0}$ and P5.

\subsubsection{Verification of transformation accuracy.}

Equation 6.4 provides a solution for $T$ in a least squares sense, thus there may be a degree of error in the solution. To test the accuracy of $T$, it is multiplied by $A$ to give a new matrix $B^{h}$ :

$$
B^{h}=A . T
$$

which is subsequently compared to its original counterpart, $B$. Recall that $A$ and $B$ represent three-dimensional models, therefore $B^{h}$ represents a new hypothetical model, and its similarity with $B$ is measured by the Euclidean distance between them. The closer the value of the Euclidean distance is to zero, the more accurate the transformation matrix, $T$. This measure of accuracy was established for all the transformation matrices shown in Table 6.2. 


\subsubsection{Investigating relationships between LGN models and terminal field models.}

To investigate the potential relationships between the changing external structure of the LGN and the developing terminal field region within it, the transformations derived for changes between the nuclei were applied to terminal field models. The resulting hypothetical models were then compared to their actual counterparts. If the dissimilarity between these models was close to zero, then the transformation describing the changes to the LGN models also describes the changes occurring to the terminal field models.

As an example, consider the transformation matrix describing the changes between the $\mathrm{P} 0$ ipsilateral nucleus, $P 0_{i n}$, and the $\mathrm{P} 5$ ipsilateral nucleus, $P 5_{i n}$. From Table 6.1, this would be defined as $T_{i n}^{P 0 \rightarrow P 5}$. If this transform is applied to the model of the terminal field within the ipsilateral nucleus at $\mathrm{P} 0, P 0_{i p}$, then the resulting hypothetical model would be of the terminal field region within the LGN at P5, P5 $5_{i p}^{h}$ :

$$
P 5_{i p}^{h}=P 0_{i p} \cdot T_{i n}^{P 0 \rightarrow P 5}
$$

The Euclidean distance between this model and its original counterpart, $P 5_{i p}$, may then be used as a measure of how well the changes to LGN structure describe the changes to the terminal field region.

The above was repeated for P5, P8, and P12 terminal field models both ipsilateral and contralateral to the injected eye. The Euclidean distance between each resulting hypothetical model $\left(P 5_{i p}^{h}, P 8_{i p}^{h}, P 12_{i p}^{h}, P 5_{c p}^{h}, P 8_{c p}^{h}, P 12_{c p}^{h}\right)$ and the actual mean model was calculated to determine the effectiveness of LGN changes in describing terminal field changes. 


\subsection{Results.}

\subsubsection{Mean models of the developing terminal fields.}

Figures 6.1, 6.2, and 6.3 on the following pages show the models generated for nuclei (wireframe models) and for terminal field regions (coloured solid models) of ipsilateral and contralateral projecting RGC fibres at P5, P8, and P12. In each column labelled 'ipsilateral', the solid models represent the regions of the right LGN occupied by label following an injection of HRP into the right eye. In the columns labelled 'contralateral', the solid models represent the regions of the left LGN not occupied by label following the same injection of HRP (i.e. this is the presumptive region occupied by fibres from the un-injected eye). In each case, models from three different animals are shown, together with the resulting mean. Although not shown here, models were also generated for P0 nuclei. Since both projections occupy the entire nucleus on both sides of the brain at this stage, the model of the ipsilateral nucleus also served as the model of the ipsilateral terminal field region. This also means that there is no region void of label in the nucleus contralateral to the injected eye, therefore there is no model of the projection region at this stage for this nucleus.

At P5, terminal fields of ipsilaterally projecting fibres occupy a large proportion of the nucleus. They occupy a smaller region of the nucleus ventrally, but extend anteriorly in more dorsal regions (Figure 6.1, Ipsilateral). By P12, the terminal field region has transformed into a symmetric elongated structure lying in the caudal end of the nucleus (Figure 6.3, Ipsilateral). Simultaneously, the figures of the nucleus on the opposite side of the brain show an area void of label at P5 expanding from a very small region in the caudal aspect of the LGN (Figure 6.1, Contralateral), to a region at P12 comparable in size and shape to that of the ipsilateral terminal field (Figure 6.3). 

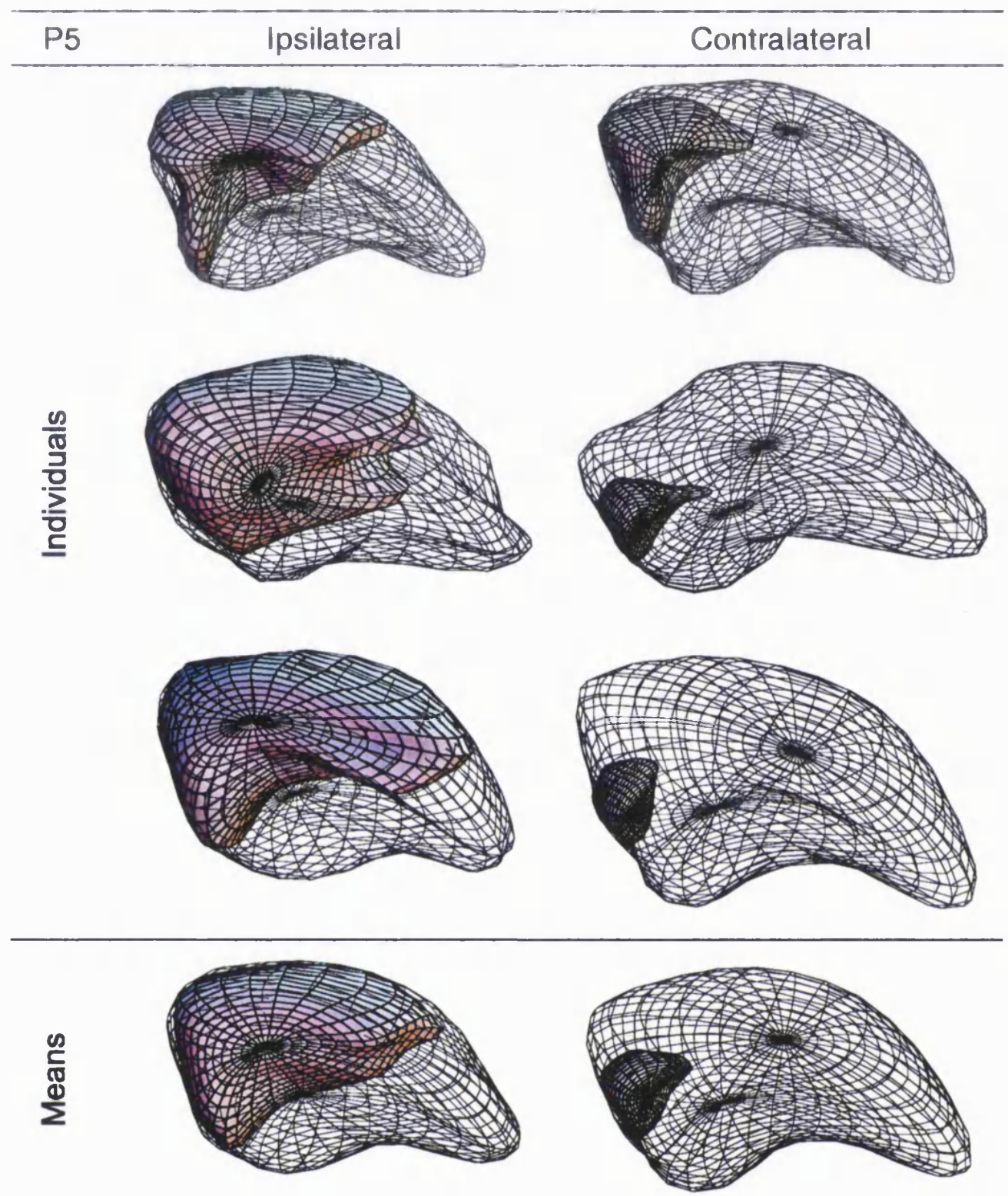

Figure 6.1 - Models showing the contralateral and ipsilateral geniculate nuclei of P5 ferrets, and their

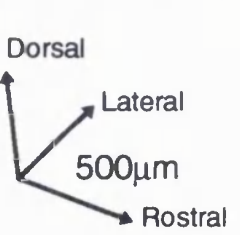
respective ocular projection areas following an intraocular injection of HRP into the right eye. For comparative purposes, all nuclei have been oriented the same way. The first three rows represent models constructed from three different animals, while the final row shows mean models. Ipsilateral to the injection (left column), the solid mass within each wire frame is the region of label. Contralateral to the injection (right column), the solid mass in each wire frame represents the region void of label. Scale and orientation is indicated on the left. Ipsilaterally, terminal label occupies a large proportion of the nucleus - it occupies a smaller region ventrally, but expands rostrally in more dorsal regions. Contralaterally, label occupies the majority of the nucleus, except for a small area caudally. Interestingly, this non-labelled region is also smaller ventrally, and expands in a rostral direction further dorsally. 


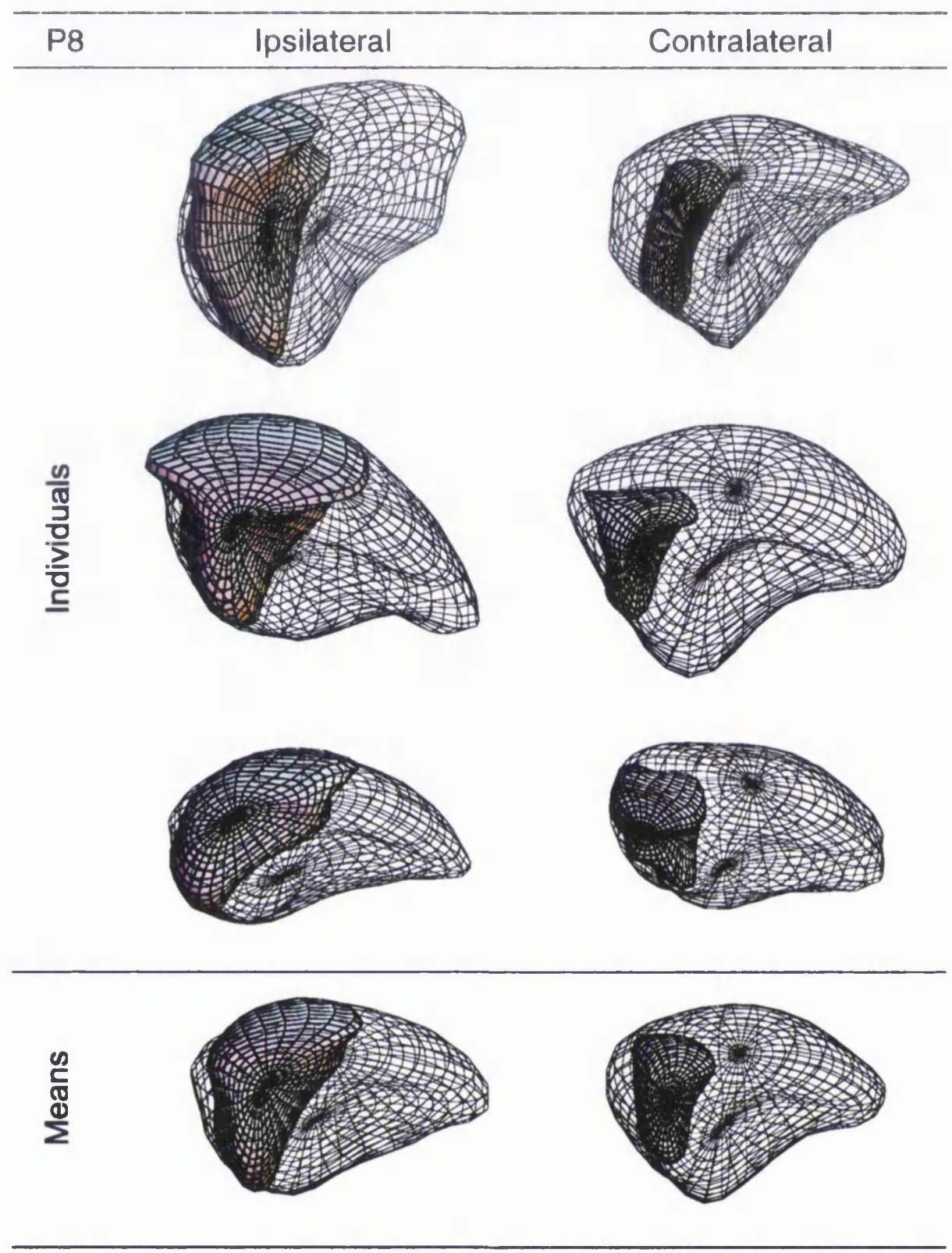

Figure 6.2 - Models showing the contralateral and ipsilateral geniculate nuclei of P8 ferrets,

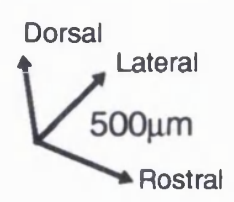
and their respective ocular projection areas following an intraocular injection of HRP into the right eye. All other conventions similar to Figure 6.1. By $\mathrm{P} 8$, the volume of ipsilateral label is reduced compared to P5, and the region void of label contralaterally has increased. There is a degree of variation both in terms of shape and orientation contralaterally and ipsilaterally, however, the resulting mean shapes of the nuclei are comparable. Similar to P5, the labelled and non-labelled regions taper ventrally. 

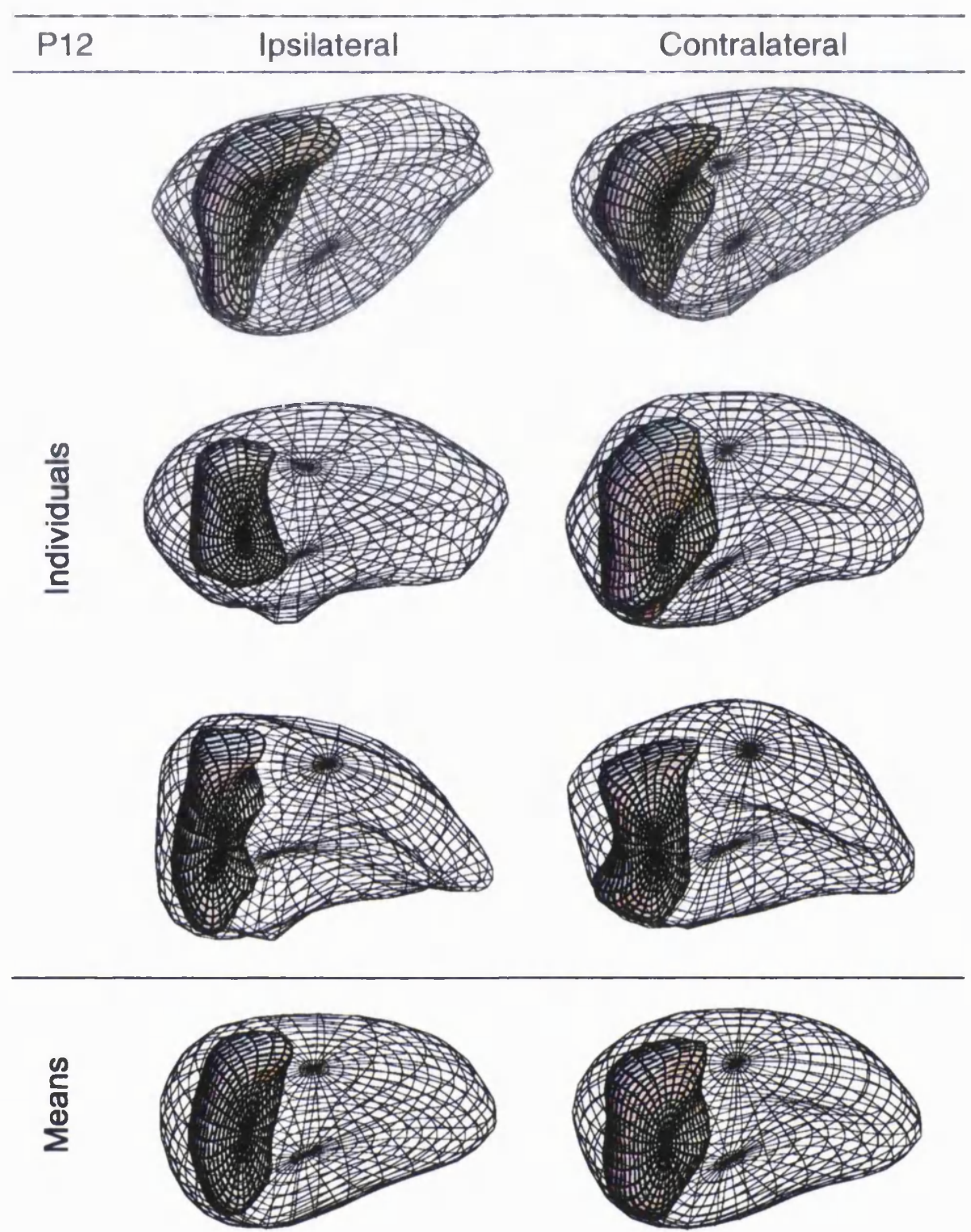

Figure 6.3 - Models showing the contralateral and ipsilateral geniculate nuclei of P12 ferrets,

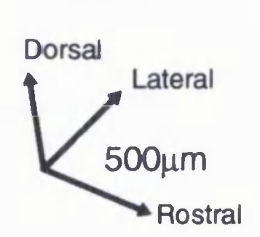
and their respective ocular projection areas following an intraocular injection of HRP into the right eye. All other conventions similar to Figure 6.1. The figure shows that by P12, the region of label in the ipsilateral nucleus is very comparable to the region of non-label in the contralateral nucleus. The implication here is that RGC axons from each eye are terminating in appropriate areas. The shape of the labelled/non-labelled region is no longer tapered, and has transformed into an elongated tubular structure spanning the LGN dorsal to ventral in the caudal portion. 


\subsubsection{Analysis of the relationship between changing LGN structure and the development of ocular segregation.}

The following section examines the relationship between changing LGN shape and the development of ocular distinct regions within it. If transformations occurring to the nucleus between two time points are the same (or similar) as those occurring to the terminal field regions, then it is possible that the two events are related. Note that in this section, any reference to the contralateral terminal field actually refers to the solid models shown in the 'contralateral' column of Figures 6.1-6.3 which represent the terminal field region contralateral to the injected eye void of label.

\subsubsection{Evaluation of the accuracy of the transformation matrices.}

The transformation matrices referred to in Section 6.2.2. were calculated as described, and Figures $6.4 \mathrm{a}-6.4 \mathrm{c}$ illustrate the accuracy of these matrices. Three columns of information are provided in each figure. The first (left column) shows a bar chart which quantitatively illustrates the dissimilarity between the two models shown in the second and third columns. Respectively, these models are the equivalent of $B$ and $B^{h}$ as described in Section 6.2.3. The Euclidean distance (or dissimilarity index) between the original model, and the hypothetical model is shown in the chart by the white bar. If the two models were exactly the same, this bar would indicate a value of zero. Conversely, any degree of dissimilarity between the models results in an Euclidean distance having any value greater than zero. Therefore, to place this quantity in context, the three grey bars show the Euclidean distance between the three individual models and the mean models at a corresponding age as was shown in Figures 6.1-6.3. This can be considered as illustrating the degree of variation in shape between individuals. Finally, the horizontal line which runs across the chart is the 
mean of these three bars, and is often referred to in the text as the mean normal

variation.
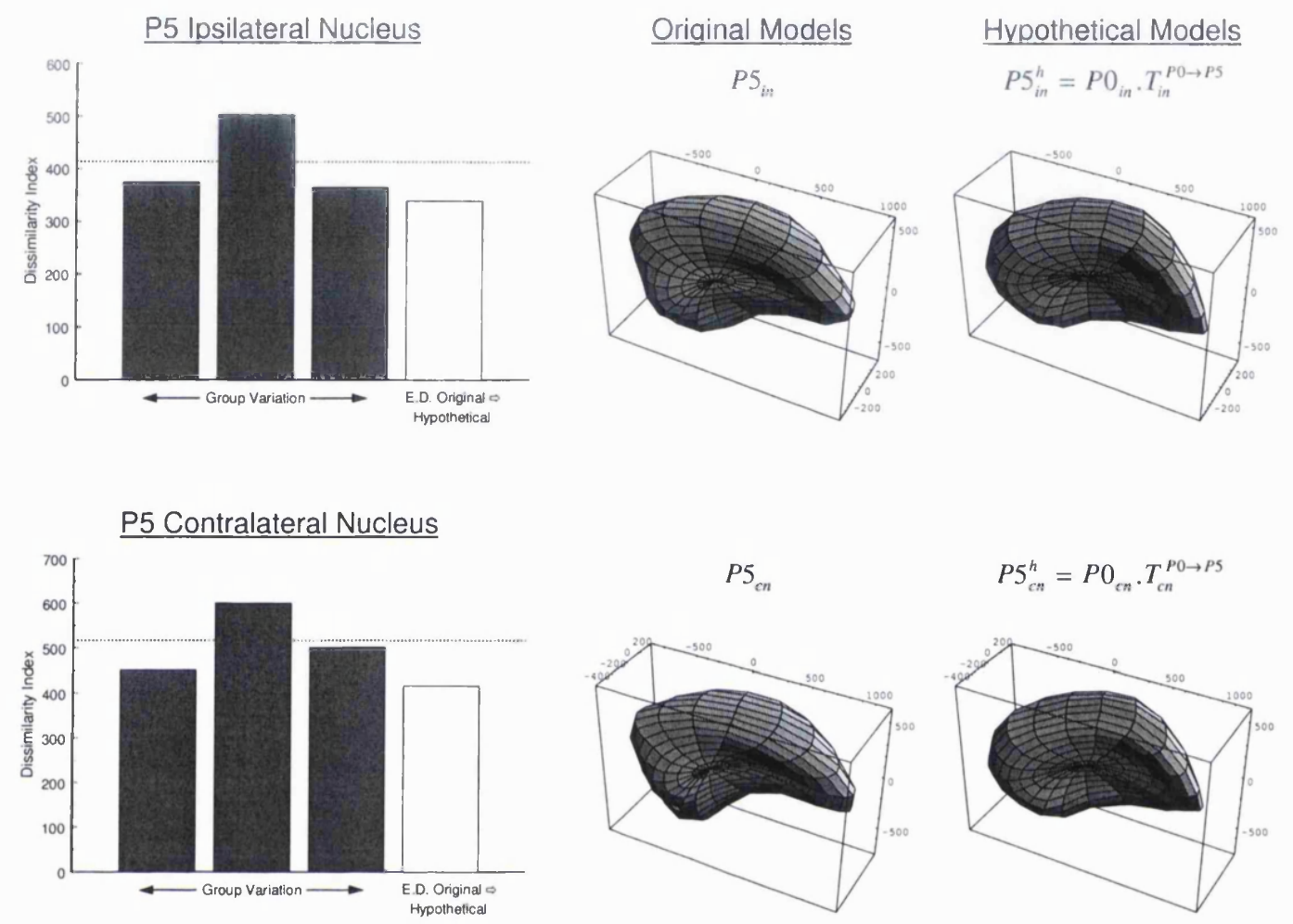

Figure 6.4a - Accuracy of the transformation matrices in describing changes between P0 and P5 LGN models. Having calculated the transforms between P0 and P5 models, they are applied to the P0 models to create hypothetical P5 models (shown in the right column). The Euclidean distances between these models and the original P5 mean models (shown in the centre column) is calculated to establish the accuracy of the transformations. These dissimilarity measures are plotted in the left column (white bars) along with the Euclidean distances between the models from individual animals and the mean models (grey bars). The horizontal line indicates the mean dissimilarity of the grey bars. Orientation of the 3D structures is the same as in Figures 6.1-6.3. The figure shows that no exact transformation could be calculated between the P0 and P5 LGN models, although the hypothetical models resemble the original, and have a dissimilarity within the range of normal variation.

It is apparent from the figures that no exact transformation could be calculated between any two time periods between any two LGN models. If this had been the case, then the white bars in each plot would have been zero (since there would be no dissimilarity between the original and hypothetical models). This does not imply 
however that the transformations could not yield plausible hypothetical models. The dissimilarity index between the hypothetical models and the mean models is lower than the mean normal variation in every case, and substantially so for the changes between P5 and P8 (Figure 6.4b) and P8 and P12 (Figure 6.4c). Comparison of the plots representing the hypothetical models in Figures 6.4a-6.4c (right column) with their original counterparts in the centre column of the same figures shows there to be good resemblance between the structures.
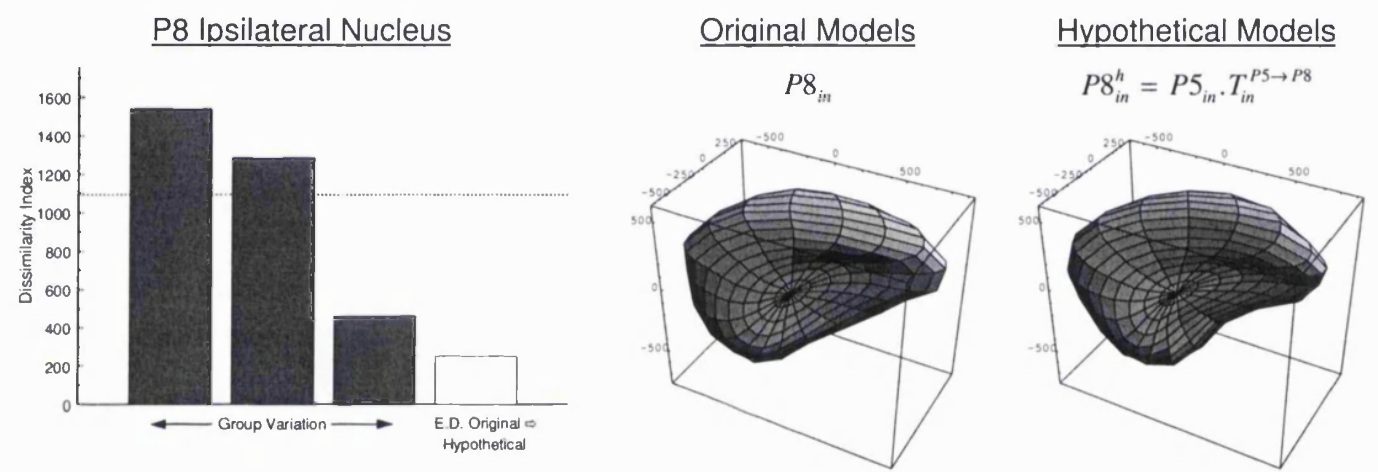

$\underline{\text { P8 Contralateral Nucleus }}$
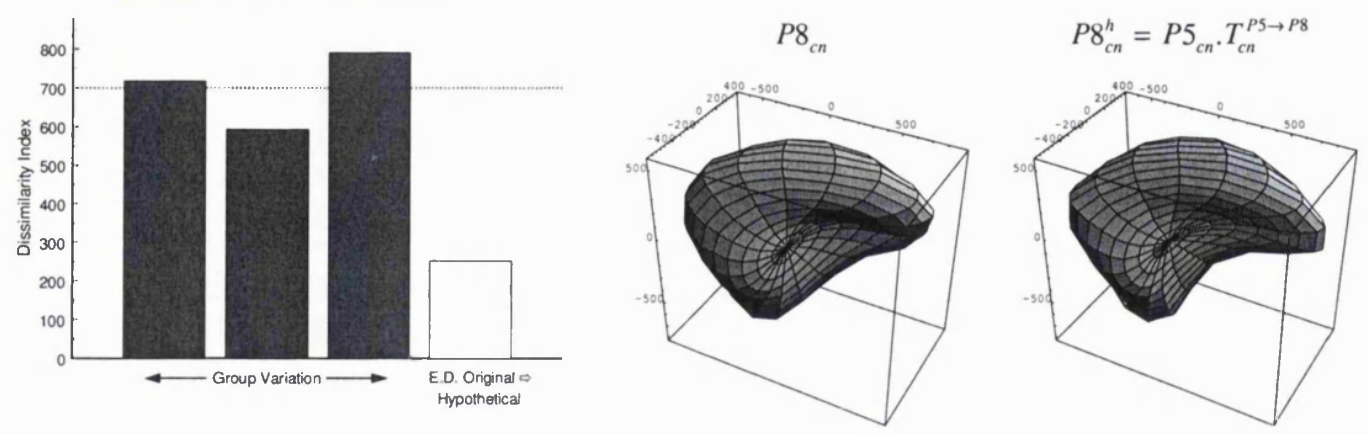

Figure 6.4b - Accuracy of the transformation matrices in describing changes between P5 and P8 LGN models. See Figure 6.4a for a full description. Dissimilarity indices indicate that exact transformations could not be established, but that hypothetical models were substantially more similar to the mean model than the individuals. 

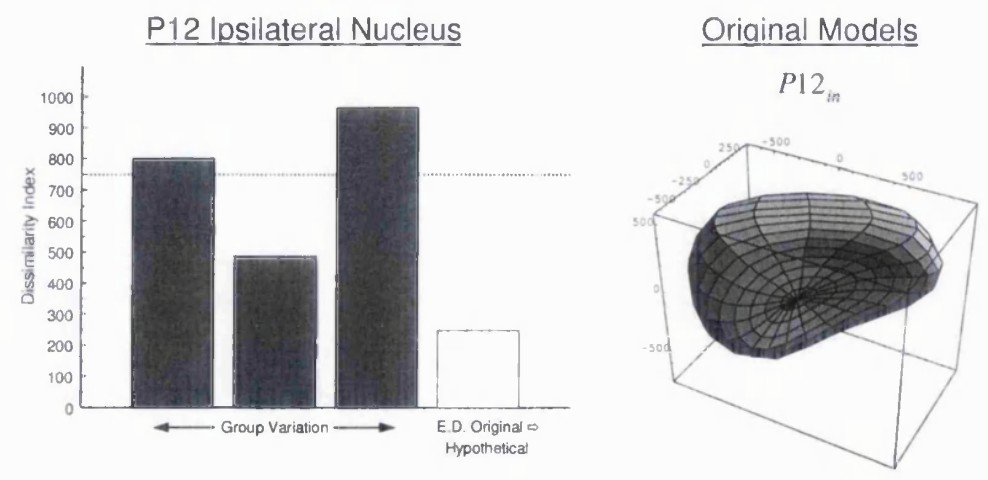

Hypothetical Models $P 12_{\text {in }}^{h}=P 8_{\text {in }} \cdot T_{\text {in }}^{P 8 \rightarrow P 12}$
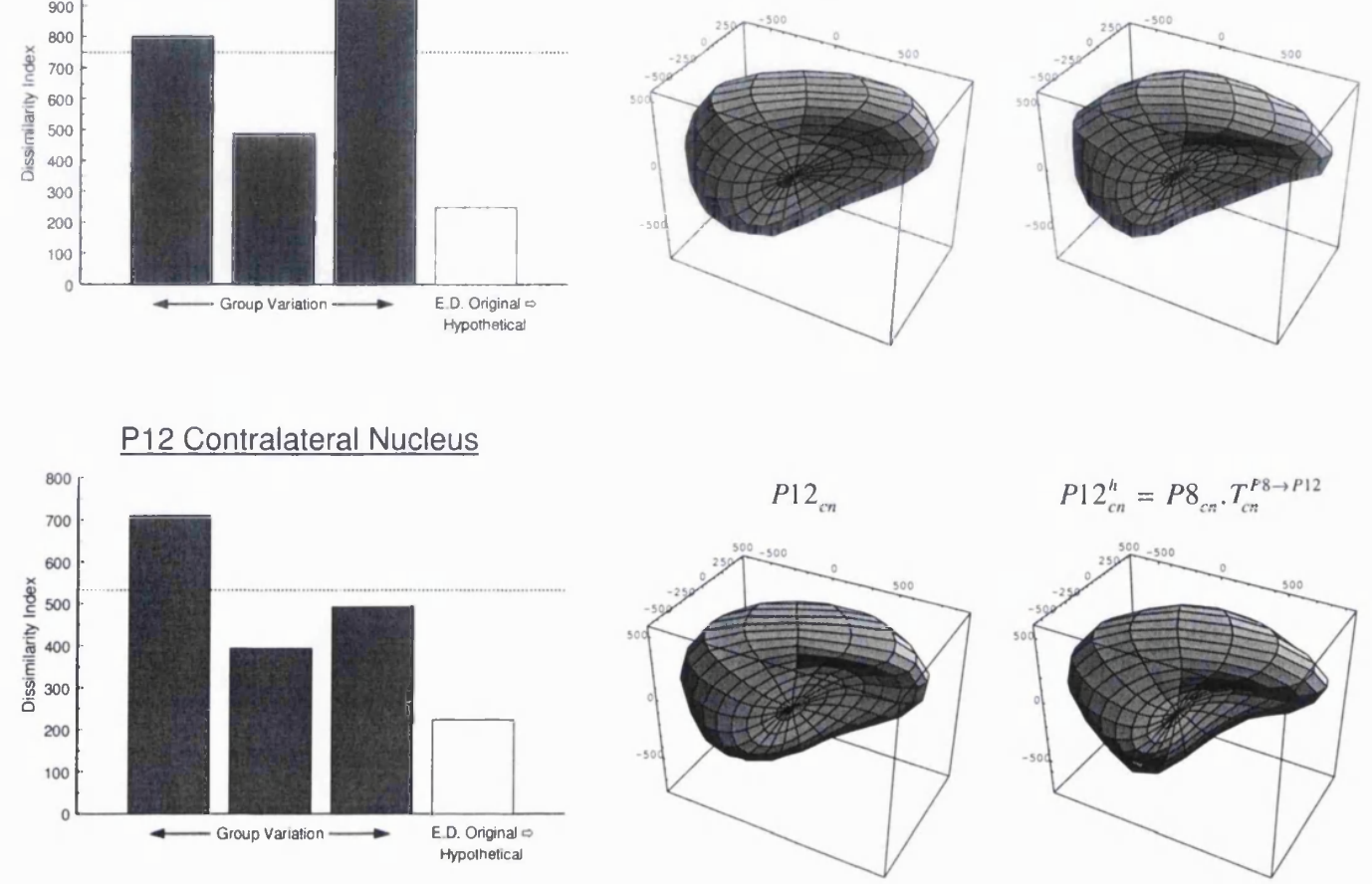

Figure 6.4c - Accuracy of the transformation matrices in describing changes between P8 and P12 LGN models. See Figure 6.4a for a full description. Again, no exact transformation could be defined for the changes occurring between the P8 and P12 nuclei.

\subsubsection{Transformations of the $L G N$, and how thev effect changes in their respective} terminal field regions.

Having calculated transformation matrices between nuclei, these have been applied to their respective terminal field models. The resulting hypothetical terminal field models were compared with their original counterparts to establish how well the changes in LGN structure reflect changes in terminal field structure. The results are summarised in Figures 6.5a-6.5c.

Between P0 and P5, the hypothetical P5 terminal field does not resemble the original model, and is visually more similar to the P5 nucleus (Figure 6.5a). The dissimilarity index of the hypothetical ipsilateral terminal field $\left(P 5_{i p}^{h}\right)$ is almost twice 

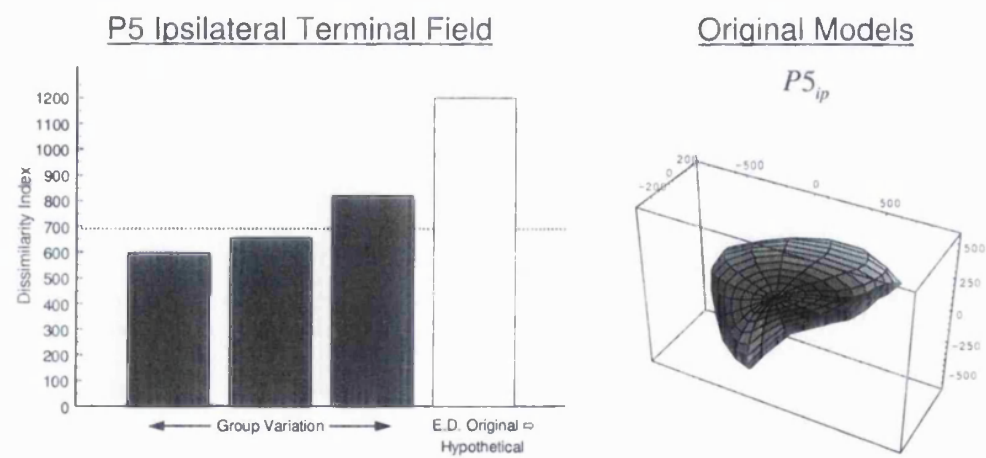

Hvoothetical Models $P 5_{i p}^{h}=P 0_{i p}, T_{i n}^{P O \rightarrow P S}$

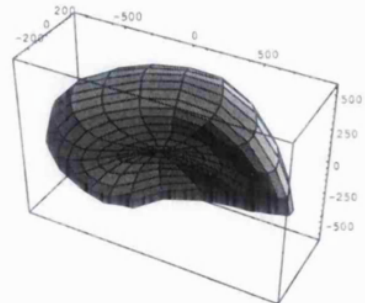

Figure 6.5a - Resulting shapes of P5 ipsilateral terminal field when the transformation between the P0 and P5 nucleus is applied to the respective P0 terminal field. The right column shows the hypothetical P5 model, and the centre column the original P5 model for comparison. The Euclidean distance between these two models is shown by the white bar in the left column. In order to put this quantity in context, the grey bars show the variation in Euclidean distance between the individual models which comprise the mean shape shown in the centre column. Orientation of the 3D structures is the same as in Figure 6.1.

the mean dissimilarity between individuals. Recall that no model for the contralateral terminal field was defined for $\mathrm{P} 0$ (i.e. $P 0_{c p}$ ), as there is no region void of label in the nucleus contralateral to the injected eye at this stage. Therefore, the associated hypothetical model $\left(P 5_{c p}^{h}=P 0_{c p} \cdot T_{c n}^{P 0 \rightarrow P 5}\right.$ ) for the opposite side of the brain is not shown.

Figure 6.5 b shows the hypothetical ipsilateral terminal field $\left(P 8_{i p}^{h}=P 5_{i p} \cdot T_{i n}^{P 5 \rightarrow P 8}\right)$ has a dissimilarity index approximately equal to the mean normal variation. However, the model appears larger than the original, and resembles a scaled version of $P 5_{i p}$. Similarly, the hypothetical contralateral terminal field structure resembles the P5 terminal field structure rather than that of the P8 animal, although the dissimilarity index again has a value around the mean of the individuals. 

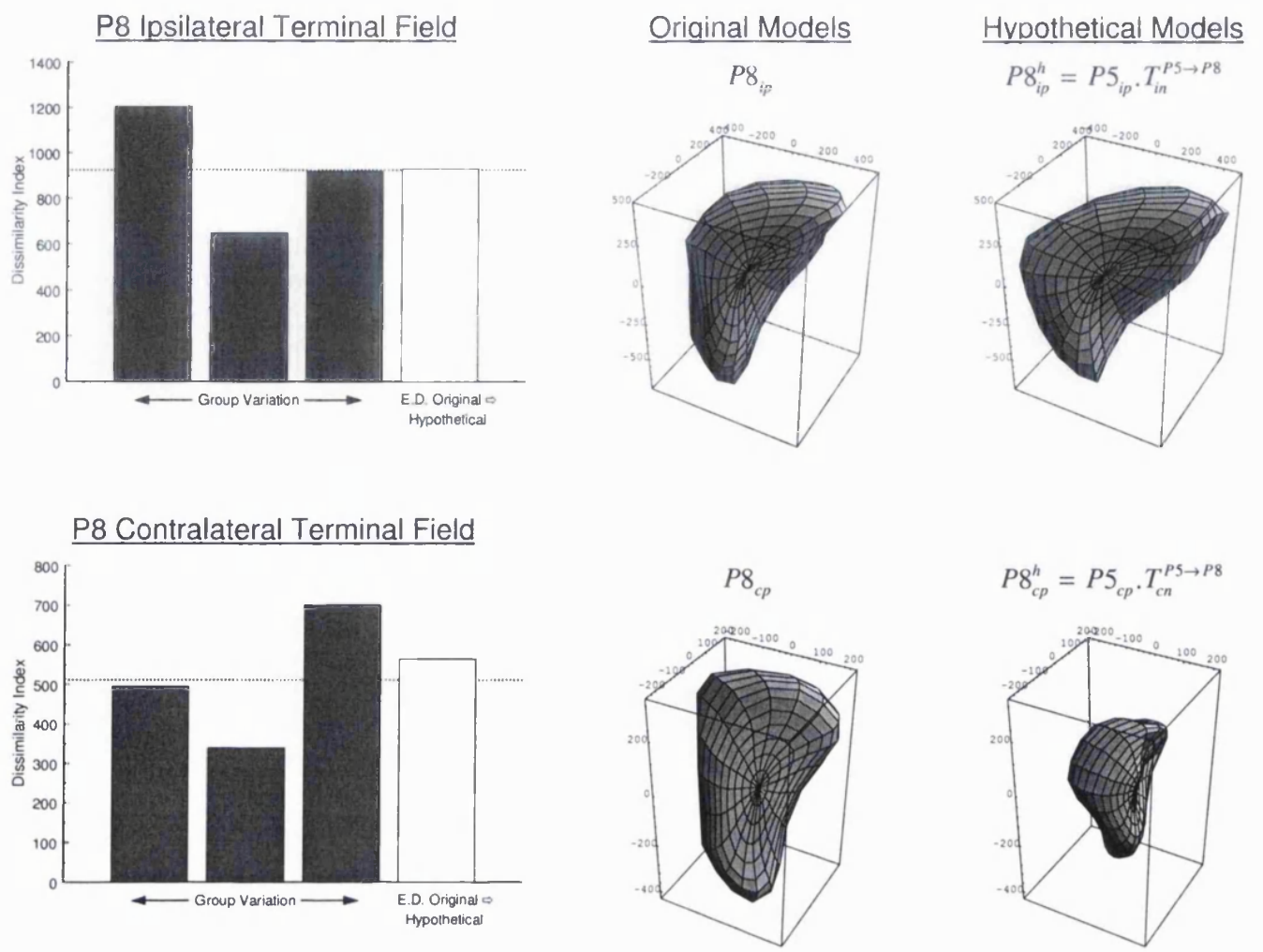

Figure 6.5b - Resulting shapes of P8 ipsilateral and contralateral terminal fields when the transformations between the P5 and P8 nuclei are applied to their respective P5 terminal fields. The right column shows the hypothetical P8 models, and the centre column the original P8 models for comparison. All other conventions as in Figure 6.5a.

P12 hypothetical structures are shown in Figures $6.5 \mathrm{c}$. The results indicate that in both cases, the resulting hypothetical models are more similar to the original P8 models rather than the P12 models. The dissimilarity between $P 12_{i p}^{h}$ and $P 12_{i p}$ is over twice the mean dissimilarity between individuals, although this probably reflects the increased scale of this model. Conversely, $P 12_{c p}^{h}$ has a dissimilarity index similar to that measured between individuals, and this is assumed to be due to the reduced scale of this model.

The main observation arising from these results is that the hypothetical models are similar to models at an earlier age rather than models at an equivalent age. This either reflects an inability of the transformation matrices to capture the elements of shape 

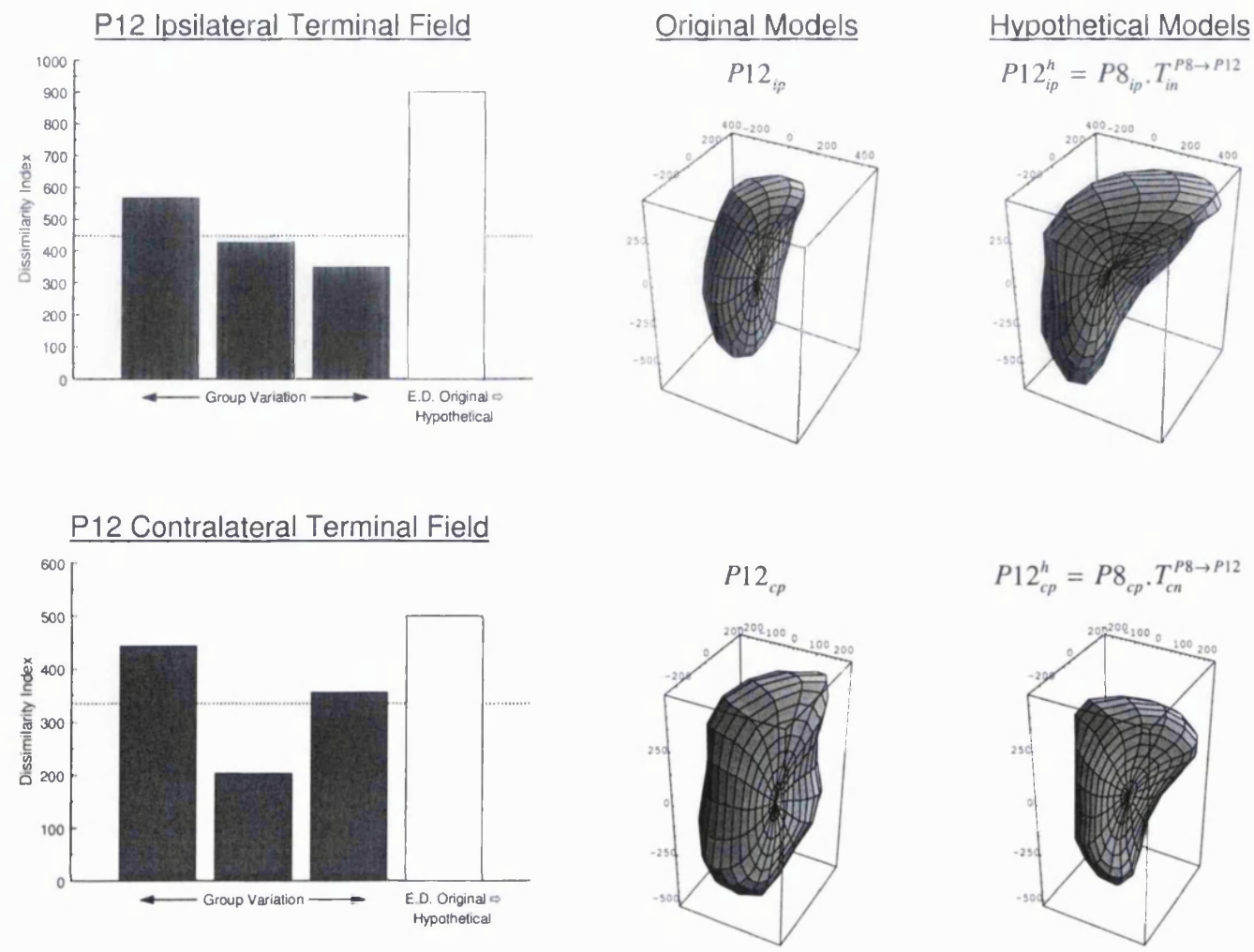

Figure 6.5c - Resulting shapes of P12 ipsilateral and contralateral terminal fields when the transformations between the P8 and P12 nuclei are applied to their respective P8 terminal fields. The right column shows the hypothetical P12 models, and the centre column the original P12 models for comparison. All other conventions as in Figure 6.5a.

change within the nuclei between two time points, or illustrates that there is very little shape change between nuclei during the ages studied. It may be the case that the results reflect the fact that the transformation may be non-linear. It could also indicate that changes in the shape of the nucleus do not reflect changes in the shape of the terminal fields. Despite this, certain gross aspects of shape change appear to be represented by the transformations, in particular scale.

The method of shape description employed here expresses shape in terms of its various spatial frequency components. The first degree harmonic provides information on gross features of shape, while subsequent harmonics provide information on the finer detail. To preserve information about finer detail, it may be necessary to establish 
the transformation between each spherical harmonic rather than employ a single transformation matrix for the entire set of spherical harmonics (as presented here). This is explored in the next section.

\subsubsection{Further detailed transformations of the LGN, and how they effect changes in the} terminal field reaions.

The above analysis was repeated by calculating a transform for every individual spherical harmonic instead of a single transform for the whole set of harmonics. Therefore, eight transformation matrices were calculated for changes between two models between each age range. Despite breaking up the analysis in this manner, the transformational relationship between ages remains linear. The results are shown in Figures 6.6a-6.6c.
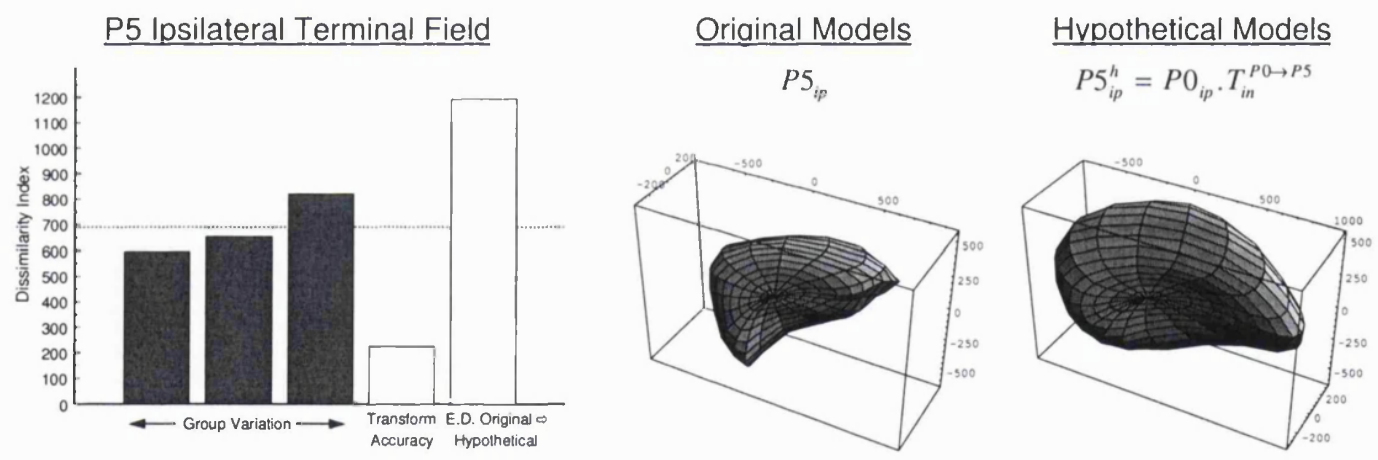

Figure 6.6a - Resulting shape of the P5 ipsilateral terminal field when the eight transformations describing changes between the $\mathrm{P} 0$ and $\mathrm{P} 5$ nucleus are applied to the respective $\mathrm{P} 0$ terminal field. The right column shows the hypothetical P5 model, and the centre column the original P5 model for comparison. The Euclidean distance between these two models is shown by the white bar in the left column marked 'E.D. Original $\Leftrightarrow$ Hypothetical'. The bar marked 'Transform Accuracy' shows how well the eight transformation matrices describe the changes between the nuclei (see Figure 6.4a). The grey bars show the variation in Euclidean distance between the individual models which comprise the mean shape shown in the centre column. Orientation of the 3D structures is the same as in Figure 6.1. While the use of a single transform between each spherical harmonic has improved the accuracy in quantifying the changes between P0 and P5 nuclei, the hypothetical models have a dissimilarity index very similar to that achieved through the use of one global transform (compare with Figure 6.5a). 
The bars marked 'Transform Accuracy' in each bar chart show that the use of a transformation matrix between each individual harmonic provides a more accurate description of the changes between nuclei at each stage (compare with Figures 6.4a6.4c). There is also a reduction in the Euclidean distances between the hypothetical terminal field models and their original counterparts, although these are not substantial. Visual examination of the hypothetical models show that very little change has been introduced through the use of eight transforms as compared with one.
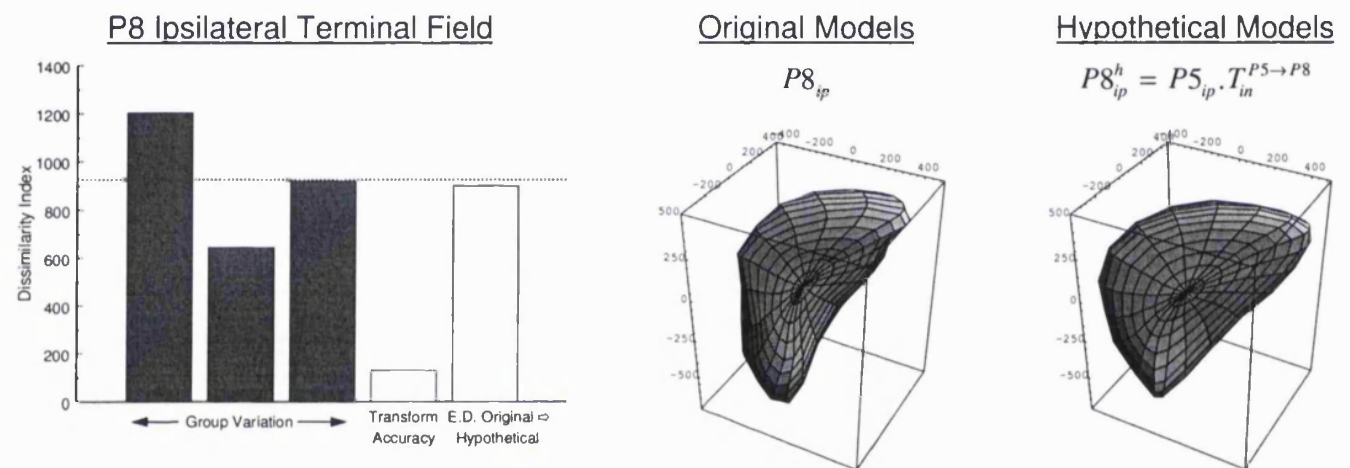

\section{P8 Contralateral Terminal Field}
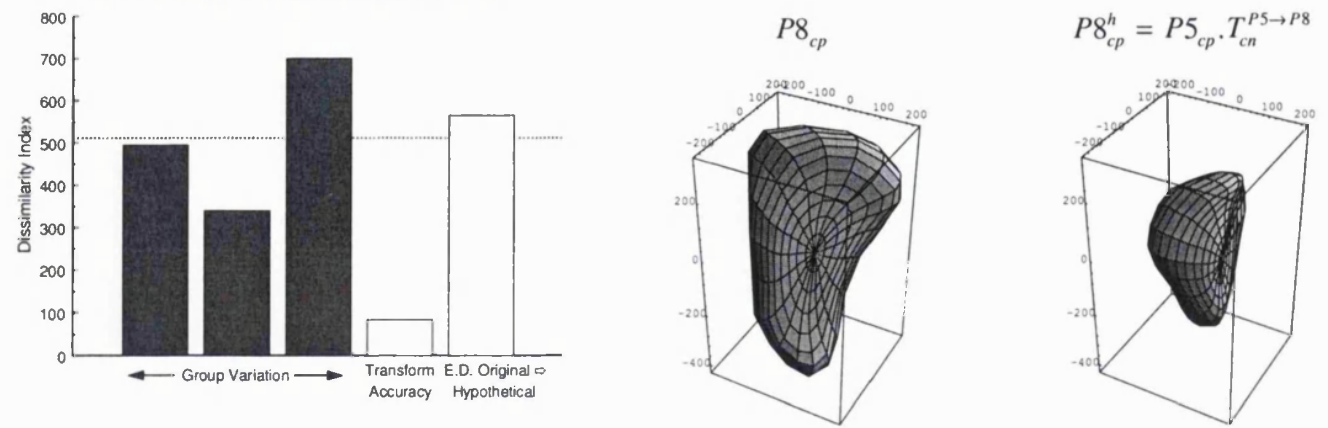

Figure 6.6b - Resulting shapes of P8 ipsilateral and contralateral terminal fields when the eight transformations describing changes between the P5 and P8 nuclei are applied to their respective P5 terminal fields. The right column shows the hypothetical P8 models, and the centre column the original P8 models for comparison. All other conventions as in Figure 6.6a. 

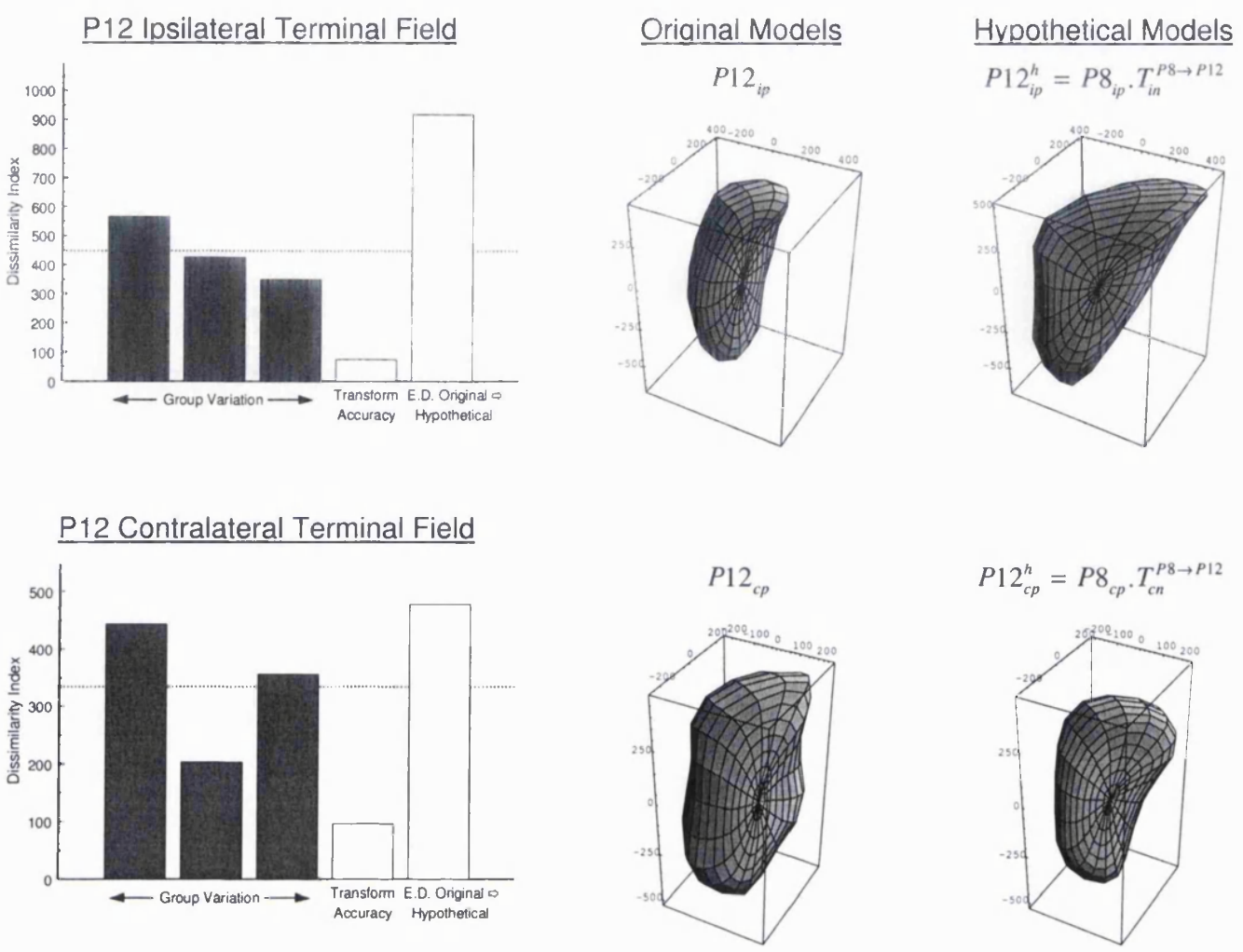

Figure 6.6c - Resulting shapes of P12 ipsilateral and contralateral terminal fields when the eight transformations describing changes between the P8 and P12 nuclei are applied to their respective P8 terminal fields. The right column shows the hypothetical P12 models, and the centre column the original P12 models for comparison. All other conventions as in Figure $6.6 a$.

\subsubsection{Spatial relationships between changing LGN structure and ocular distinct terminal field structure.}

The previous section examined global relationships between the developing LGN shape and the terminal field within it. Here, specific local spatial relationships between the two structures are explored by examining the displacement vectors that describe their changes between two time periods.

The protocol of analysis described in Section 5.2.3 was employed to calculate displacement vectors between the mean LGN models, and their respective terminal field structures between P0 and P5, P5 and P8, and P8 and P12. The areas of greater expansion and contraction between LGN structures were similar to those described in 
Section 5.3.5. For illustrative purposes, Figure 6.7 shows the resulting vectors between the P5 and P12 nuclei (compare with Figure 5.15).
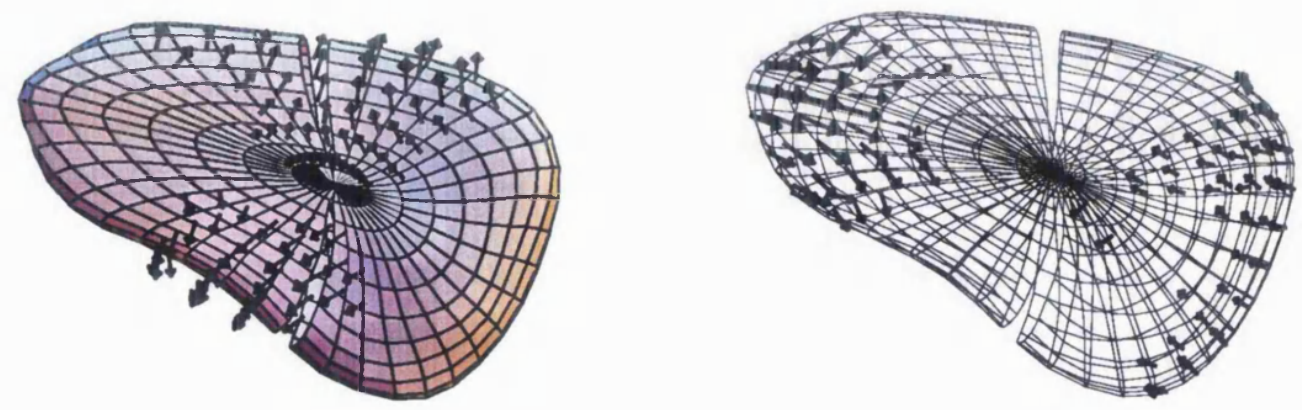

Figure 6.7 - Vectors showing areas of greater expansion (solid figure) and contraction (wire figure) between P5 and P12 nuclei. The nucleus shown is the mean P5 model, and the vectors illustrate the main changes in shape required to transform the P5 nucleus to the mean P12 structure. The results are consistent with those shown in Figure 5.15 between the P5 and P10 nuclei. Both plots show the medial surface of the LGN, and rostral is towards the left.

In comparing changes between nuclei (as illustrated above), models were normalised to account for the rotational displacement that the nucleus undergoes (see Section 5.3.2). To perform the corresponding comparisons between the respective terminal field structures, the same normalisations applied to the LGN models were applied to the terminal field models.

\subsubsection{Relationship between specific changes in LGN shape and the corresponding terminal field reqion ipsilateral to the iniected eve.}

Figure 6.8 shows the displacement vectors between the ipsilateral terminal field regions for the ages indicated. The changes occurring to the shape of the terminal field region were considerably larger than those occurring to the LGN at all the stages analysed. The mean vector length for overall changes to the terminal field structure between P0 and P5, P5 and P8, and P8 and P12 was approximately twice that seen to occur to the nucleus at the same ages. 


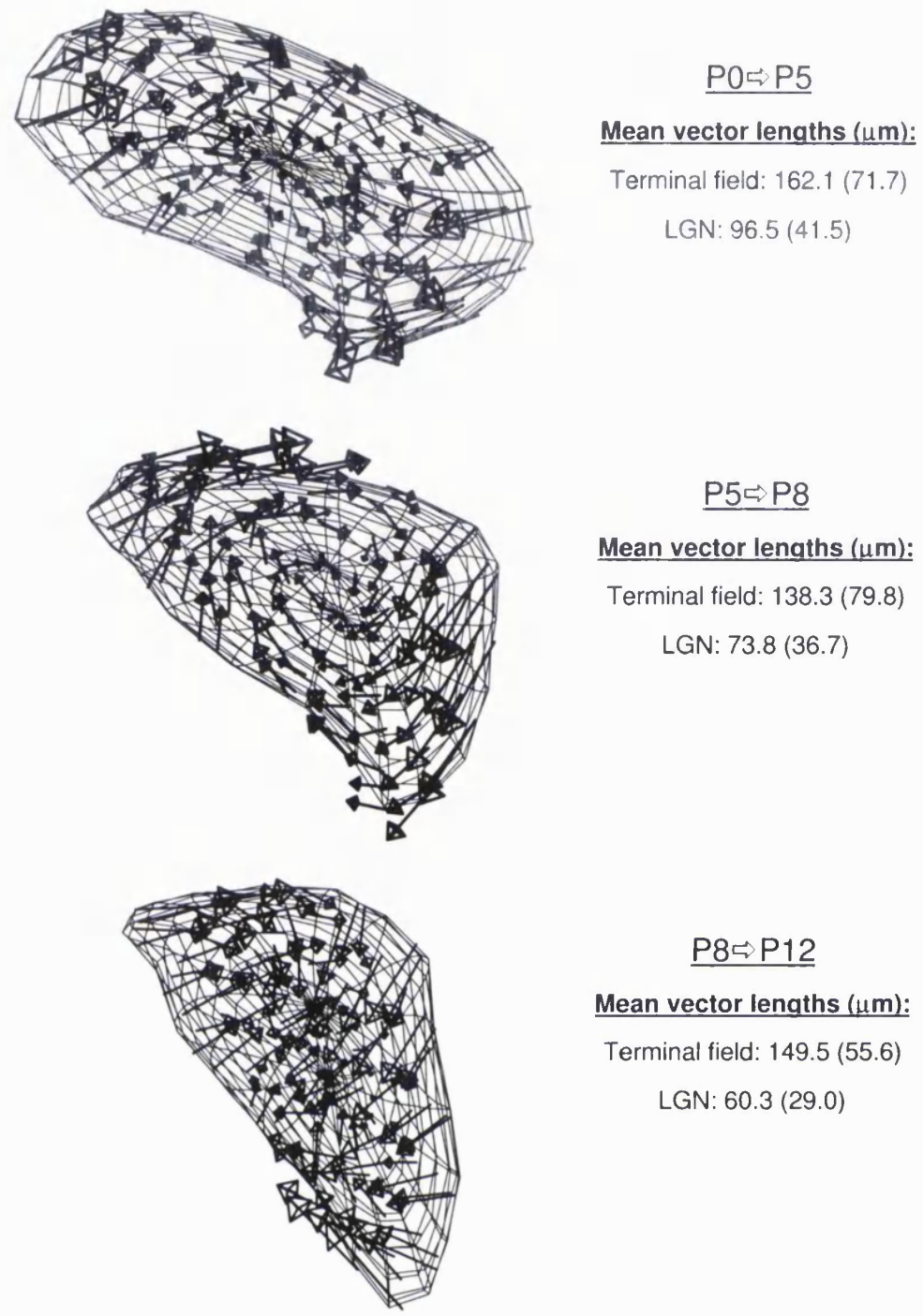

Figure 6.8 - Vectors showing spatial changes between ipsilateral terminal field regions at the ages indicated. Each figure shows the terminal field region at the earliest age in each range, together with vectors describing the changes to the later age in the range. The medial surfaces of the terminal field regions are shown, and rostral is towards the left. Data show the mean length (\& SD) of vectors. Specific local changes to the terminal field regions are approximately twice those measured in the LGN.

Comparisons of the general directions of change in Figure 6.8 with those showing changes between $\mathrm{P} 0$ and $\mathrm{P} 10$ in Chapter 5 (Figures $5.14 \& 5.15$ ) provide conflicting results. Between $\mathrm{P} 0$ and $\mathrm{P} 5$, the LGN has been shown to expand along the rostrocaudal axis (Figure 5.14), but Figure 6.8 shows the terminal field region to contract in this axis. However, the directions of change between the P5 and P10 nuclei (Figure 
5.15) are more consistent with the changes in the terminal fields. An expansion of the superior and inferior surfaces of the LGN, combined with a contraction of the rostral tip of the nucleus could be envisaged as inducing the changes in the terminal fields as observed between P5 and P12.

To establish any quantitative relationships between vectors describing spatial changes between structures, their magnitude and direction were compared. Magnitudes of corresponding vectors are compared by calculating the differences between them. Directions of corresponding vectors are compared by calculating the angle between them. Angles which tend towards $0^{\circ}$ imply that vectors have the same direction, while those which tend towards $180^{\circ}$ are in opposite directions to one another. Corresponding vectors are those vectors which have the same position on the surface of the models as defined by the two parameters $\theta$ and $\phi$ (see Section 2.5).

Figure 6.9 shows the difference in magnitude and direction between corresponding vectors describing changes between nuclei, and those describing changes between terminal field regions (as shown above) ipsilateral to the injected eye. The height (i.e. $z$-axis) of each plot determines the differences between vectors, while the $x$ - and $y$-axes determine the position of the vector on the model (i.e. $\theta$ and $\phi$ ). For the differences in magnitude, positive values for $z$ indicate that the changes to the terminal field region are greater than those for the LGN. If the magnitudes of the vectors between nuclei were the same as those between terminal field regions across the entire surface of the models, then the plots would show flat surfaces centred at $z=0$. Similarly, if the vectors were all in the same direction, the plots would show flat surfaces centred at $z=0$. 

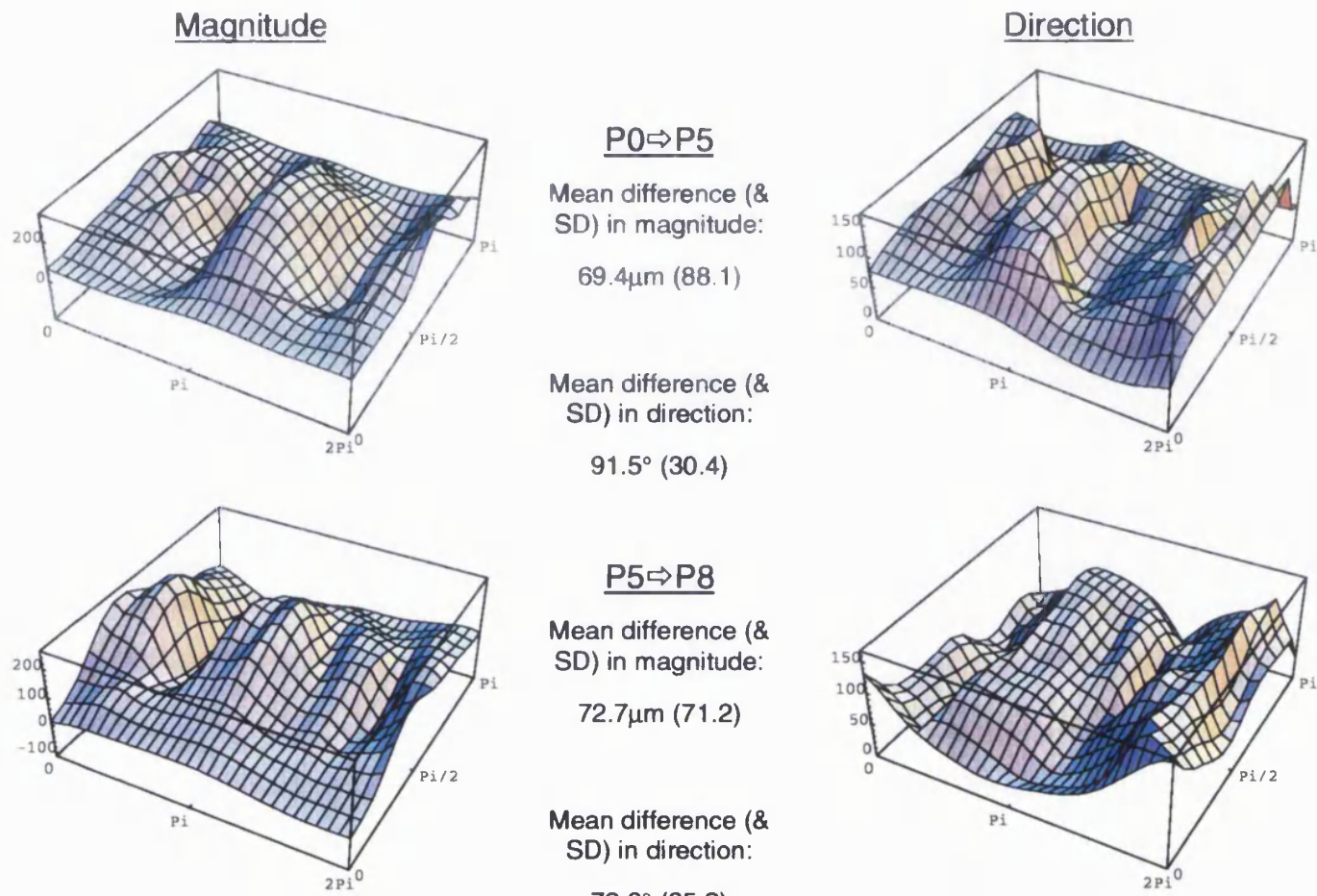

\section{$P 5 \Rightarrow P 8$ \\ Mean difference $(\&$ $\mathrm{SD})$ in magnitude:}

$72.7 \mu \mathrm{m}(71.2)$

Mean difference $(\&$ $\mathrm{SD})$ in direction:

$72.6^{\circ}(35.2)$
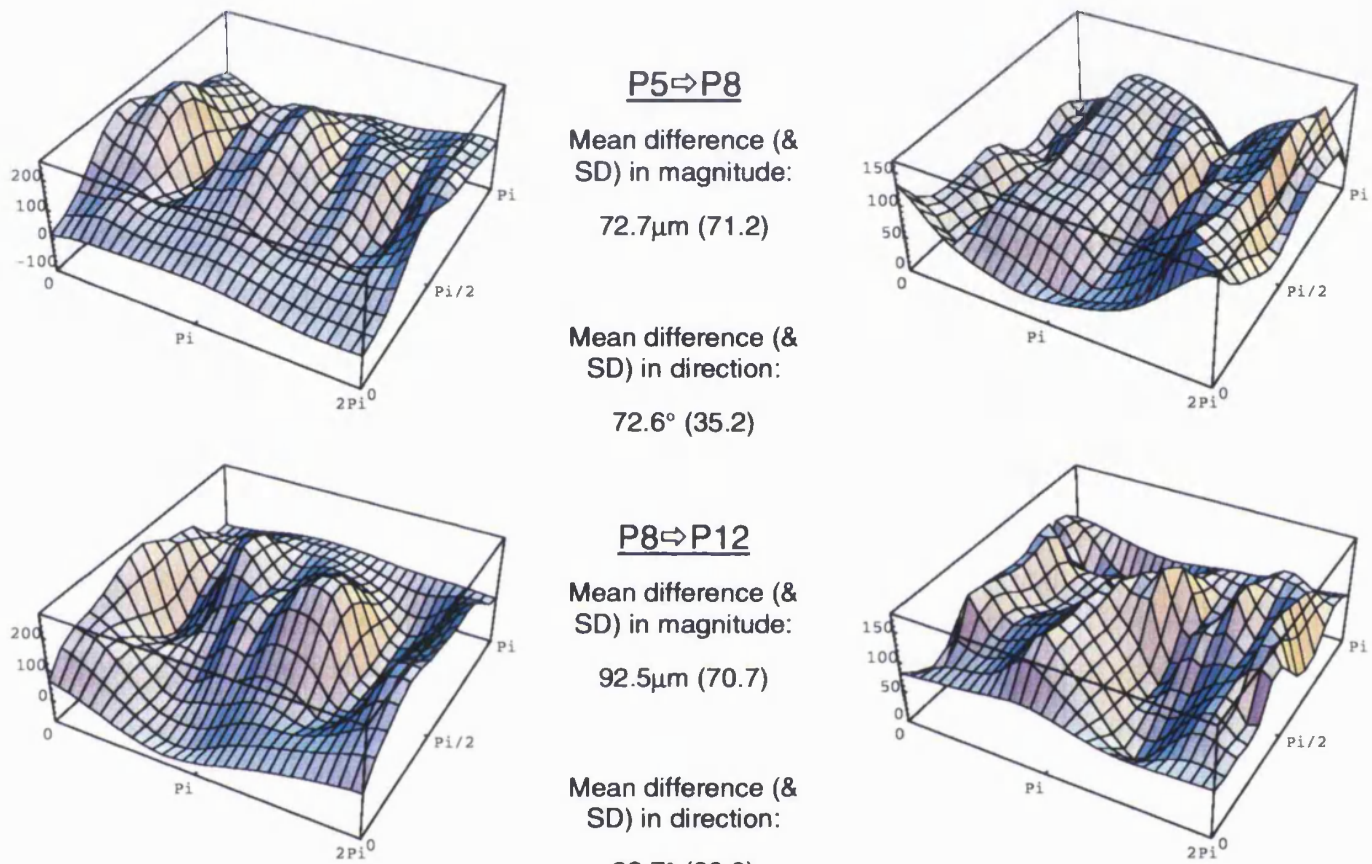

\section{$\underline{\mathrm{P} 8 \Rightarrow \mathrm{P} 12}$}

Mean difference $(\&$ $\mathrm{SD})$ in magnitude:

$92.5 \mu \mathrm{m}(70.7)$

$$
\begin{aligned}
& \text { Mean difference ( } \& \\
& \text { SD) in direction: } \\
& 92.7^{\circ}(36.0)
\end{aligned}
$$

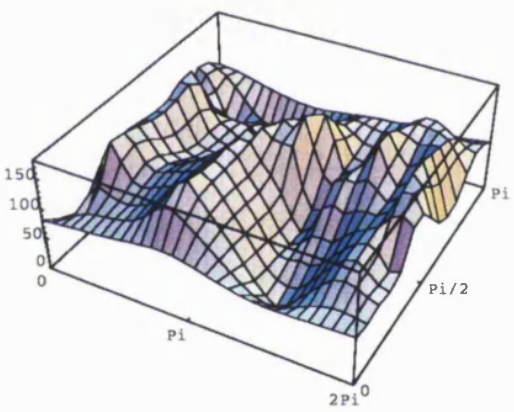

Figure 6.9 - Plots illustrating the difference in magnitude and direction between vectors describing changes to the LGN, and corresponding vectors describing changes to the terminal field regions ipsilateral to the injected eye, and between the ages indicated. The $z$-axis shows the magnitude differences (left column) or direction differences (right column), while the $x$-and $y$-axes indicate the position of the vectors on the models. Note that the colour representation is for shading purposes only.

For all age ranges, there is no uniform relationship between the direction of the vectors describing the specific shape changes to LGN and those describing the changes to terminal field regions. This is not surprising considering the qualitative observations made above for changes between P0 and P5. However, for the later age ranges, it might have been expected that the mean difference in direction between vectors would be lower than actually measured. Closer scrutiny of Figures 5.15 and 
6.8 reveal that the results are probably representative. For example, the direction of vector changes on the caudal aspect of the LGN are generally rostral and dorsal, but in the same area of the terminal field, they are rostral and ventral, i.e. perpendicular to one another.

\subsubsection{Relationship between specific changes in LGN shape and the corresponding} terminal field region contralateral to the injected eve.

Figure 6.10 shows the specific changes to the contralateral terminal field between P5 and P8, and P8 and P12. The models represent the region of the LGN contralateral to the injected eye which is void of label. No data is shown for P0 to P5 since there is no region of the nucleus void of label at P0. In contrast to events on the opposite side of the brain, the mean length of vector changes to this terminal field region are approximately the same as those occurring to the nucleus.

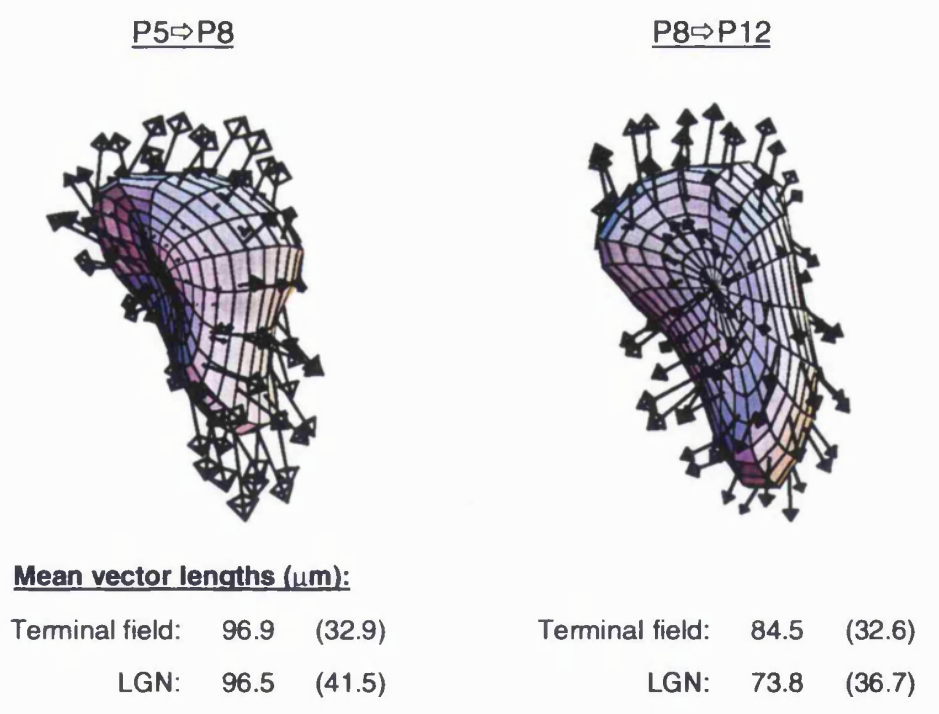

Figure 6.10 - Vectors showing spatial changes between terminal field regions in the nucleus contralateral to the injected eye at the ages indicated. Recall that the models of the terminal field regions as shown here are of the areas void of label. There is no data for the P0 to P5 range, since there is no region within the P0 nucleus void of label. Each figure shows the terminal field region at the earliest age in each range, together with vectors describing the changes to the later age in the range. The medial surfaces of the terminal field regions are shown, and rostral is towards the left. Data show the mean length (\& SD) of vectors. 
Growth of these terminal field regions is relatively isotropic, and the lack of any relationship with the direction of LGN growth is reflected in Figure 6.11. Plots of the angles between vectors show that the majority are far from being in the same direction as each other, the mean difference between them being over $90^{\circ}$.
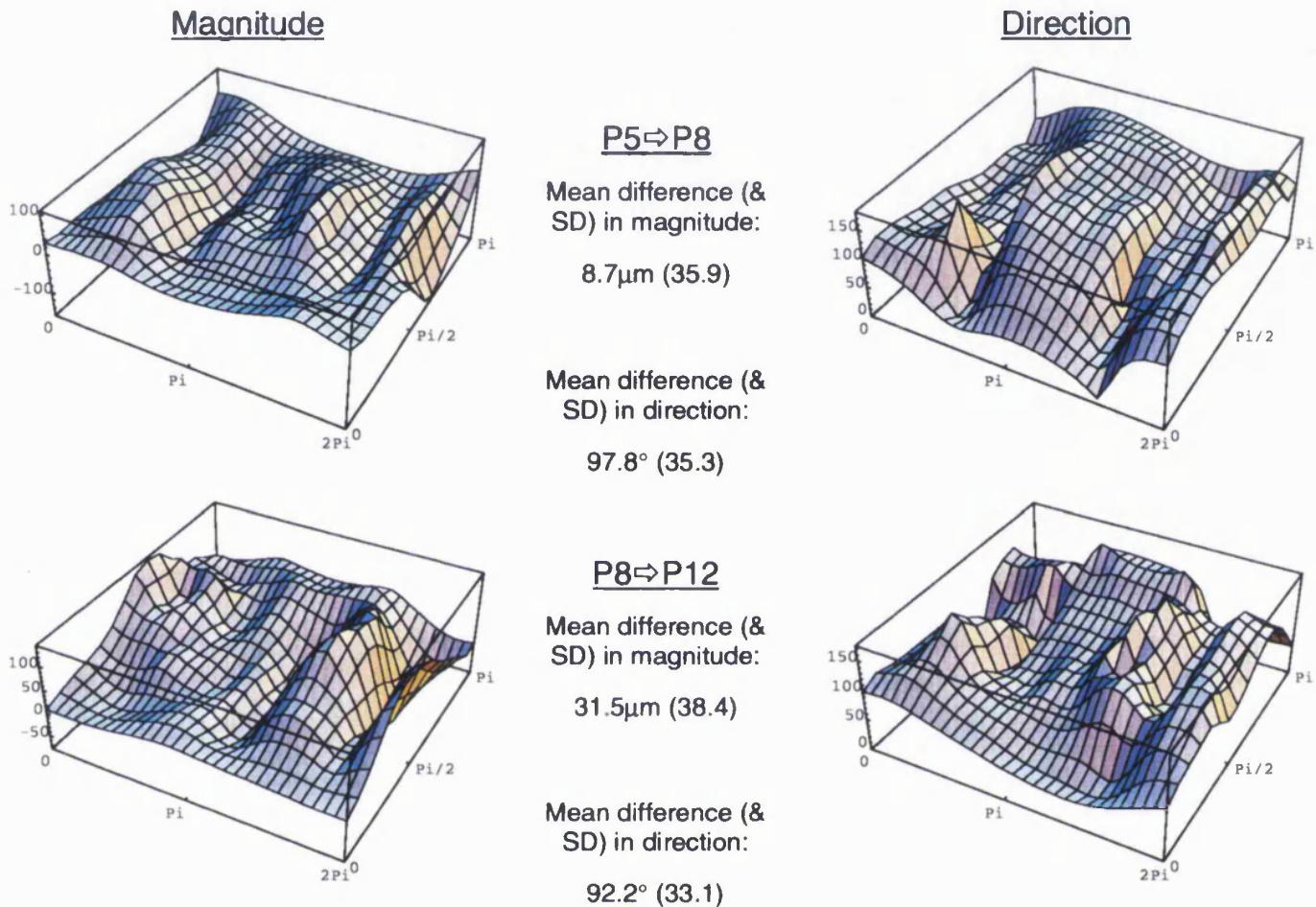

Figure 6.11 - Plots illustrating the difference in magnitude and direction between vectors describing changes to the LGN, and corresponding vectors describing changes to the terminal field regions contralateral to the injected eye, and between the ages indicated. The $z$-axis shows the magnitude differences (left column) or direction differences (right column), while the $x$ - and $y$-axes indicate the position of the vectors on the models. Note that the colour representation is for shading purposes only. 


\subsection{Discussion.}

Previous theories on the formation of eye-specific layers in the LGN have focused mainly on the role of activity-driven competitive interactions between ganglion cell axons (for review, see Shatz, 1994; see also Section 1.3). Segregation into ocular distinct regions is a common feature of RGC target nuclei, and while the patterns are the same between individuals, they are different between nuclei. For example, the segregation of retinal fibres in the SC of the ferret into eye-specific clusters (Zhang and Hoffmann, 1993) is different to that shown in the LGN in this chapter. In addition, Angelucci et al (1997) have shown that eye-specific patterns can be experimentally induced in non-visual targets of the ferret, and these again produce unique patterns. These observations indicate that activity-driven mechanisms can only be partially involved in segregation, and that other influences may be provided by the target structures themselves (whether they be on a cellular/molecular level, or on a structural level).

This chapter has explored the relationships between the developing eye-specific layers of the LGN and its structure by utilising the techniques for modelling shape described earlier. Mean shape models of the nucleus and the RGC terminal field areas have been produced, and these have facilitated an analysis of the relationship between the two structures.

\subsubsection{Do changes in LGN shape affect changes In terminal field regions?}

The method of analysing changes in the shape of the LGN as described in Section 6.3.2, and whether they reflect changes in the shape of the terminal field region cannot provide an adequate answer to the above question. First, no precise transformation 
could be established between LGN models for any two time periods. Application of approximate LGN transforms to shape models of terminal field regions did not produce hypothetical models which were visually similar to their original counterparts. However, in some cases (e.g. P8), measurement of the Euclidean distance between original and hypothetical models showed them to be within the range of normal variation.

Visually unrealistic hypothetical terminal field models are probably a result of the inherent inability of the transformation matrices to capture the precise elements of shape change between two nuclei between two time points. It is evident that if this method of comparing changes between shapes is to be pursued further, then a more accurate means of establishing shape transformations is necessary.

The measure of dissimilarity used here is evidently susceptible to the gross dimensions of the models. It is believed that those measures of dissimilarity of the hypothetical models which are within the normal range of variation, are due to scale differences between the original and hypothetical models, rather than true reflections of shape difference. Use of the Euclidean distance as a dissimilarity measure can therefore be misleading, and should probably be used in conjunction with other biological parameters (such as volume, cross-sectional area etc.), possibly as part of a multivariate analysis.

\subsubsection{Relationships between specific LGN shape change and terminal field changes.}

The data from Chapter 3 have shown that while the nucleus increases in volume during development, the volume of the ipsilateral terminal field remains the same (in a 
statistical sense). Here however, it has been shown that the terminal field regions on both sides of the brain undergo considerable shape change. Ipsilateral to the injected eye, the magnitudes of vectors describing local changes have been shown to be approximately twice that seen in the nucleus. An analysis of the magnitudes and directions of corresponding vectors describing differences between LGN shape and terminal field shape has shown there to be no uniform relationship between them. Similar results have been shown for the contralateral terminal field, except for the fact that the mean magnitude of vectors describing changes to this region are virtually the same as those of the LGN.

In an ideal scenario, displacement vectors would be calculated between corresponding biological landmarks. Unfortunately, no landmarks can be identified within the LGN or within the terminal field region at any age (with possibly one exception, the representation of the blind spot in older animals). The models themselves have therefore been used to create the landmarks by using equally spaced values of the model parameters $(\theta$ and $\phi)$ to identify points on the surfaces. Corresponding landmarks are therefore those landmarks identified with the same values of $\theta$ and $\phi$, and may not be directly comparable in a biological sense. An alternative would be to compare vector changes between locations defined by retinotopic positions, although it is difficult to envisage how this would be achieved practically. 


\section{The role of retinal afferents in regulating} cellular constituents and structure of the LGN.

\subsection{Introduction.}

The previous chapters have described LGN development in terms of structure and cellular architecture, and have explored the degree to which they influence each other. Here, the extent to which retinal afferents regulate the cellular constituents and threedimensional configuration of the nucleus are investigated by comparing the results from normal animals with those from monocularly enucleated animals.

Early eye removal can have a number of effects on the retinofugal pathway. As the crossed projection is proportionately large in the ferret (in comparison to the uncrossed: (7\% RGC, Morgan et al., 1987; Henderson et al., 1988), monocular enucleation effectively removes the majority of retinal fibres innervating the contralateral nucleus (i.e. the LGN contralateral to the enucleation). On both sides of the brain enucleation results in an increase in LGN territory available for terminals from the remaining eye, consequently supporting more RGC axon terminals, and reducing natural ganglion cell death. The increase in surviving ganglion cells is greater 
for those which project ipsilaterally, since they gain proportionately more terminal space in comparison to the crossed projection.

Guillery et al (1985) have described LGN anatomy in monocularly enucleated mink and ferrets. The nucleus ipsilateral to the enucleated eye appears relatively normal, and has a complement of layers which can be defined cytoarchitectonically. These comprise of the A and C layers seen in the normal animal, but with the A1 and $\mathrm{C} 1$ layers missing. The space normally occupied by these layers is occupied by the increased contralateral projection from the remaining eye. Contralateral to the enucleation, the nucleus is approximately $20 \%$ smaller in the mediolateral and dorsoventral planes. A complement of layers can also be identified cytoarchitectonically in this nucleus (comprising of the A1 and C1 layers), but they are far less well defined. Similar results have been documented for the cat (Chalupa and Williams, 1984) and primate (Rakic, 1981) where monocular enucleations result in simple two-layered structures with morphological abnormalities.

While monocular enucleations induce morphological changes in the LGN, it has been shown that functionally, the nucleus is apparently normal. In cats which were enucleated prenatally, recordings from geniculate cells ipsilateral and contralateral to the enucleated eye gave normal responses to visual stimuli (Chalupa and Williams, 1984). In addition, this study showed that the retinotopic organisation was also normal: nuclei on both sides of the brain contained the representation of the zero vertical meridian at the border of the MIN and LGN, and receptive fields were located progressively laterally within the nuclei with increasing distance from the vertical meridian. Morgan (1986) reported similar findings in the ferret LGN following 
recordings contralateral to the enucleated eye. The study also showed a clear segregation of cells with ON- or OFF-type receptive fields.

In spite of the above, there has been no attempt to describe the changes in threedimensional morphology of the nucleus following enucleation, and how these vary through development. This chapter seeks to describe the changes to the LGN under these circumstances. Of particular interest is whether the developing morphology of the nucleus is dependent or independent of its normal pattern of retinal innervation. If the developing shape of the nucleus is independent of retinal input, then changes may be intrinsic to the nucleus. Alternatively, other mechanisms may be implicated, such as the innervation by corticogeniculate afferents.

The techniques employed in previous chapters to analyse aspects of the cellular population of the LGN, and the changes in shape which occur to the nucleus are applied here to the nuclei on both sides of the brain of monocularly enucleated animals. The results are compared with those of the normal unoperated animal.

\subsection{Methods.}

The methods employed for collecting and analysing data throughout this chapter have been described in Chapters 2,4 and 5. Details of the surgical procedure for monocular enucleation are provided in Section 2.2.

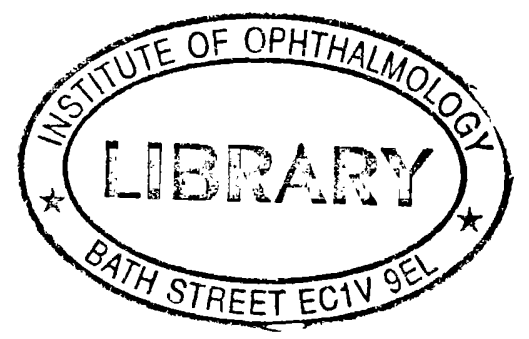




\subsection{Results.}

\subsubsection{The developing LGN in horizontal cross-section.}

The effects of a monocular enucleation on the day of birth on the developing LGN on both sides of the brain are shown in Figure 7.1 for animals at P5, P10, and P25. The P47 animal is not shown due to poor staining quality. The sections are taken from mid-way between the dorsal and ventral poles of the nucleus.

At P5, there is a symmetry to the nucleus ipsilateral and contralateral to the enucleation. Both are of a similar size, and show the characteristic shape seen in the normal animal. In the P10 animal, the nucleus contralateral to the enucleation is beginning to show a relative reduction in size, in comparison to that on the opposite side of the brain. This reduction is more noticeable 15 days later at $\mathrm{P} 25$, and greater still at P47 (not shown).

The lateral rotation of the nucleus observed in the normal animals is also present in the monocularly enucleated animal. Measurements made in the same way as described for the normal animals (Section 5.2.2) confirm that the LGN on both sides of the brain of the monocularly enucleated animal displace laterally by approximately $70^{\circ}$. The removal of a large proportion of input into the nucleus therefore appears to have no effect on this aspect of its development, indicating that LGN rotation is either a result of non-retinal factors such as the expansion of the thalamus, or an inherent mechanism of the nucleus, for instance to accommodate efficient connections within the retino-geniculo-cortical pathway. 


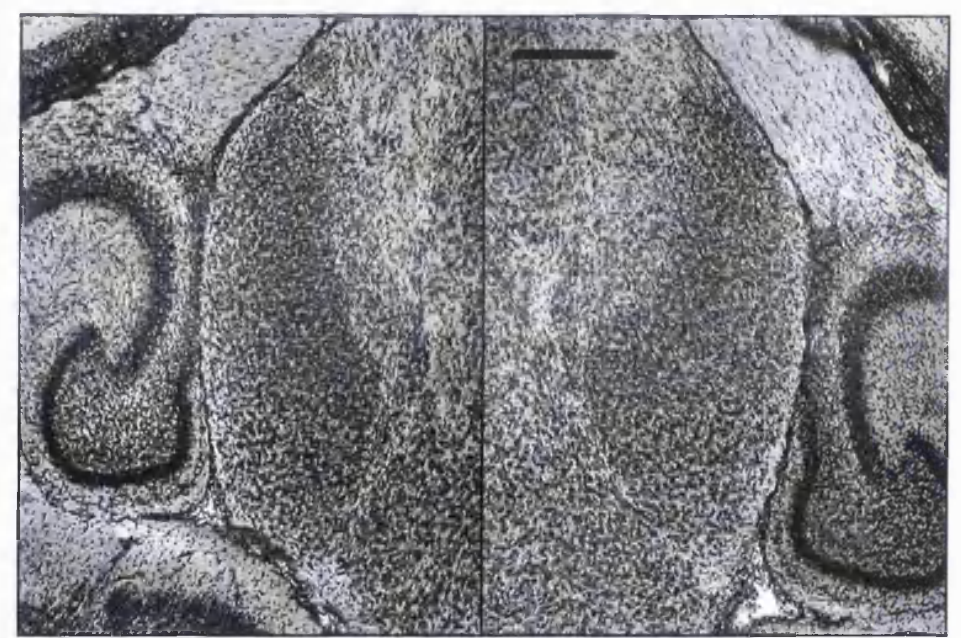

P5

Scale Bar: $250 \mu \mathrm{m}$

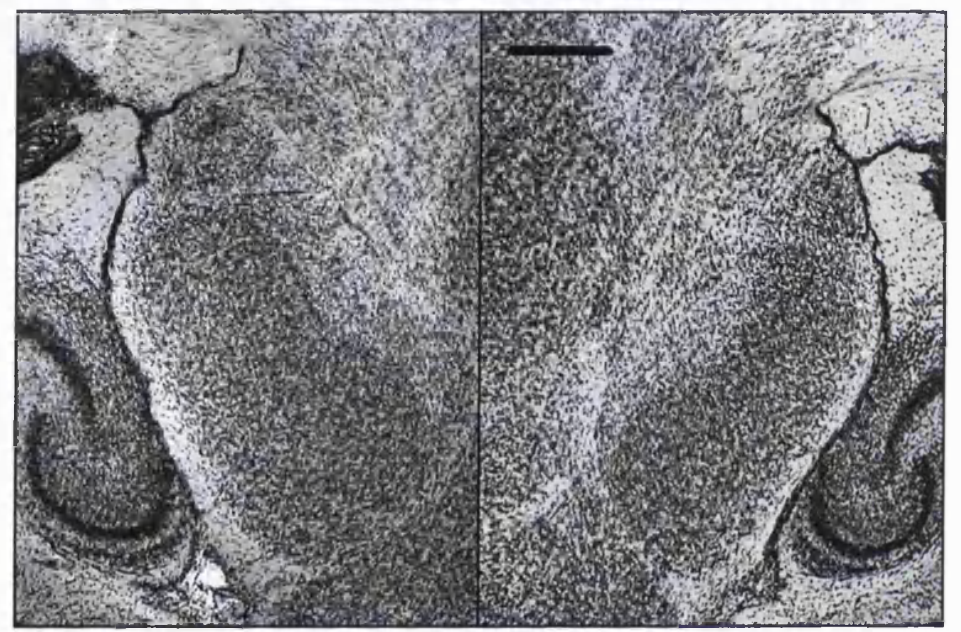

P10

Scale Bar: $250 \mu \mathrm{m}$

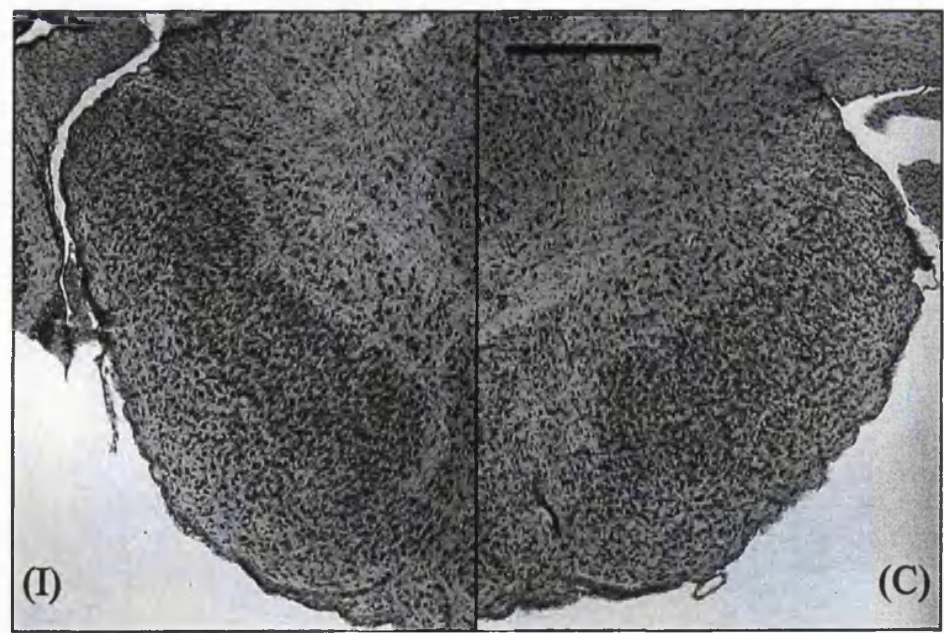

\section{P25}

Scale Bar: $500 \mu \mathrm{m}$

Figure 7.1 - Horizontal sections mid-way between the dorsal and ventral poles of the ferret LGN ipsilateral (I) and contralateral (C) to a monocular enucleation performed at P0. Rostral is towards the top of the page. A steady decrease in the size of the nucleus contralateral to the enucleation can be seen with age. The lateral rotation reported in the normal animal is also evident here. 


\subsubsection{LGN volume, cell numbers and densities.}

Figure 7.2 shows the volume of the nucleus, cell density and total cell number within the entire LGN both contralateral and ipsilateral to the enucleated eye. The plots also have the curves which were fitted to the normal data (see Figures 4.3, 4.4 and 4.5) superimposed for comparative purposes.

The results for volume as presented here (Figure 7.2a) are consistent with previous findings (Thompson et al., 1993), and show that the nucleus at maturity (P47) ipsilateral to the enucleation is similar in volume to that in the normal unoperated animal. Contralaterally, the nucleus is substantially reduced in size. This reduction is anticipated, considering that a large majority of fibres innervating this nucleus have been removed. Thompson et al (1993) have shown that the mature LGN in pigmented ferrets contralateral to enucleation is approximately $28 \%$ smaller in volume, which is similar to the data presented here. During early development, the volume of the LGN is practically the same on both sides of the brain. The growth in the nucleus contralateral to the enucleation appears to retard during the second or third postnatal week.

The density of cells both ipsilaterally and contralaterally to the enucleation are similar during all stages of development (Figure 7.2b). Additionally, the density of cells in the nuclei on both sides of the brain are similar to the normal animal. In contrast, total cell numbers in the LGNs of the enucleates differ substantially from the normal animals. The adult number of LGN cells ipsilateral to enucleation is approximately $22 \%$ lower than the normal animal, while contralateral to enucleation, adult cell numbers are approximately $46 \%$ less. The data for the contralateral nucleus also show that the peak in cell numbers may occur later than in the normal (possibly 


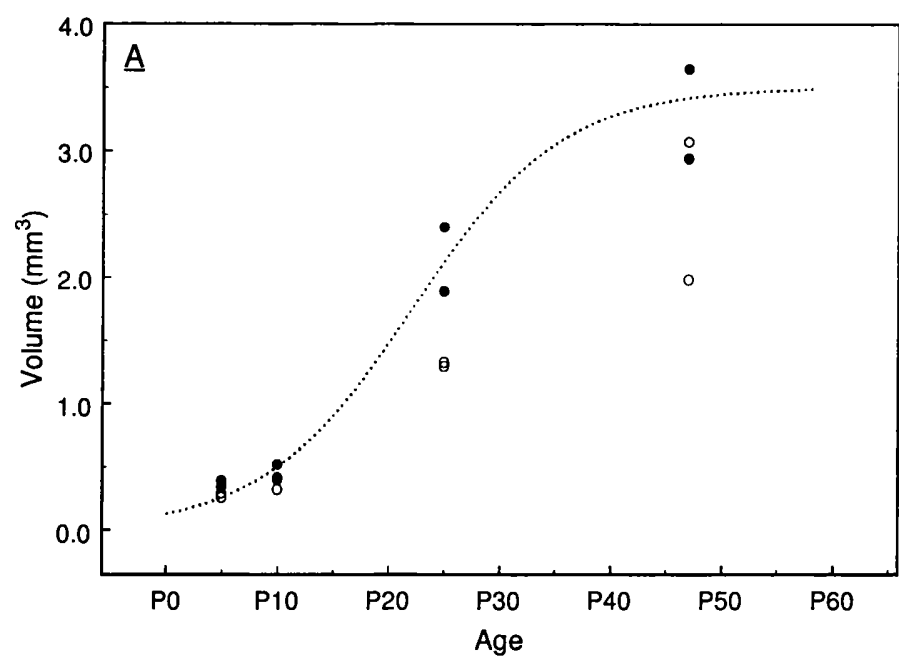

Ipsilateral

$\alpha=4.35(3.61-5.10)$

$\beta=9.91$ (5.00 - 14.81)

$\gamma=0.16(0.08-0.24)$

Contralateral

$\alpha=3.65$ (3.48 - 3.82)

$\beta=9.50$ (8.20 - 10.80)

$\gamma=0.15(0.13-0.17)$

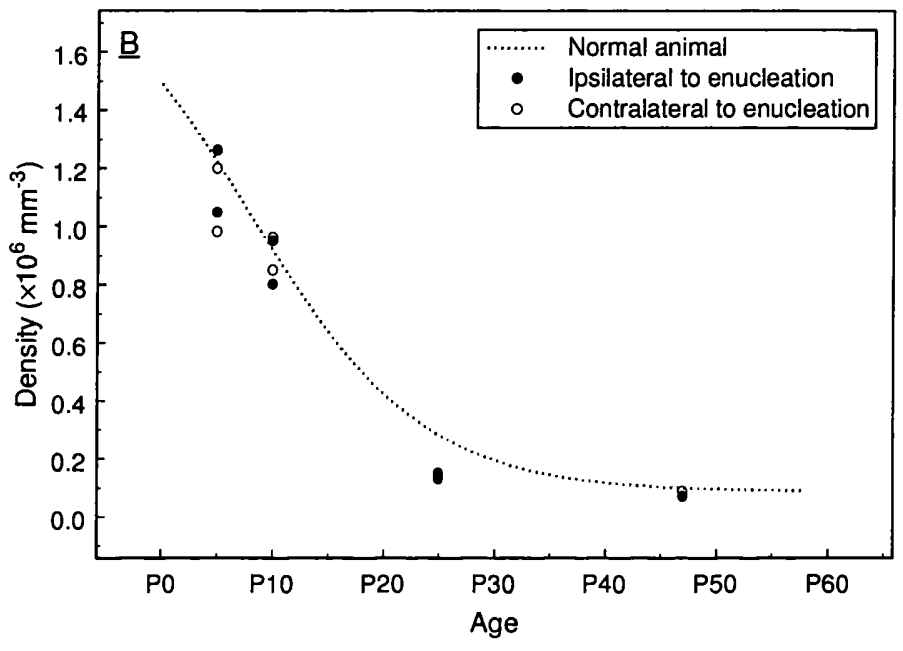

Ipsilateral

$\alpha=1.96(1.79-2.13)$

$\beta=7.14$ (3.41 - 10.88)

$\gamma=0.14(0.07-0.22)$

Contralateral

$\alpha=1.97(1.76-2.18)$

$\beta=6.42$ (2.47 - 10.36)

$\gamma=0.13(0.05-0.21)$

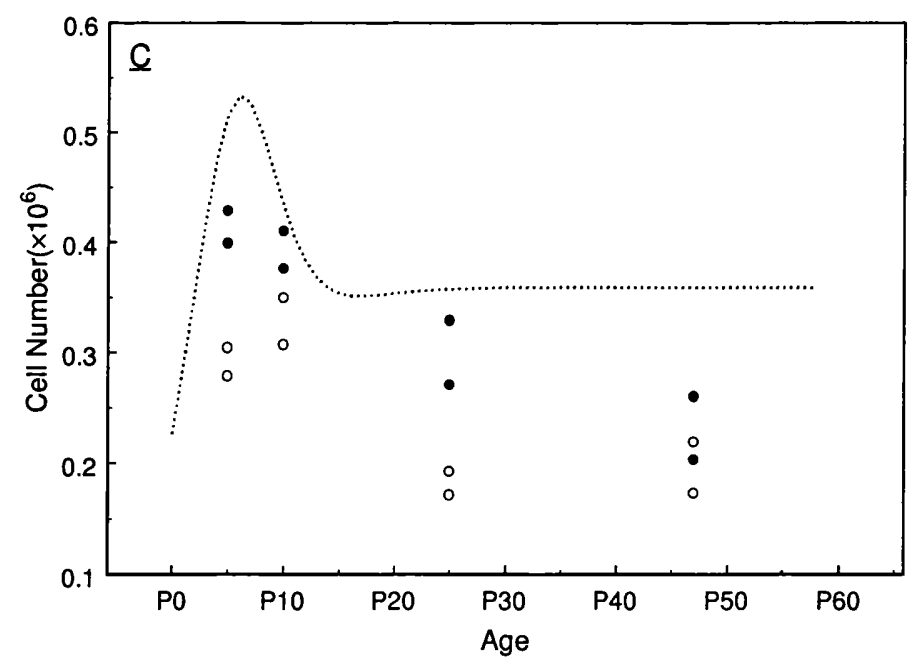

Figure 7.2 - Volume of the LGN (A), cell density (B) and total cell number (C) within the nucleus of the ferret both ipsilateral and contralateral to a monocular enucleation performed at P0. Also shown in each plot are the growth curves calculated for the normal animal (Section 4.3.2). The data on the right shows the estimates for the growth curve parameters fitted to the data shown in $\underline{\mathrm{A}}$ and $\underline{\mathrm{B}}$, together with their respective $95 \%$ confidence limits. Note that these growth curves are not shown (see Figure 7.13). 
around P10, Figure 7.2c), although there is not sufficient data from around this time to ascertain this notion.

\subsubsection{Cell size in the monocularly enucleated ferret LGN.}

The growth of LGN cells ipsilateral and contralateral to the enucleated eye is shown in Figure 7.3. Cell size was measured in terms of cross-sectional area using the methodology described in Section 4.2.3. Pooled data from all the sampled regions are shown in all the plots, each point representing the mean cell size at the age indicated, together with the associated standard deviation. The data in Figures $7.3 \mathrm{a}$ and $7.3 \mathrm{~b}$ are fitted with growth curves, and have the corresponding growth curve from the normal animals superimposed for comparative purposes (from Figure 4.10).

For all the ages investigated, mean cell size in the nucleus ipsilateral to enucleation was consistently greater than that seen contralaterally (Figure 7.3c), although there were no significant differences between them. During development, cell size in the nuclei on both sides of the brain is comparable with the normal animal, and begins to deviate (from the normal) during the third postnatal week (Figures 7.3a \& 7.3b). The temporal progress of cell growth is very similar for ipsilateral and contralateral nuclei (as illustrated by the growth curves), and in both cases, the maximum rate of cell growth is reached around the $16^{\text {th }}$ postnatal day. Whether the point at which the growth curves plateau represent the adult values cannot be ascertained from the data presented here, as data for the normal animal demonstrated a reduction in mean cell size by approximately $30 \mu \mathrm{m}$ between the oldest animal used here (P47), and the oldest animal used in Chapter 4 (P140). 
The distributions of the cell size measurements are shown in Figure 7.4, and the mean cell size is indicated by the vertical line. The shapes of the distributions are not only similar between the nuclei ipsilateral and contralateral to the enucleated eye, but also between the normal animal (compare with Figure 4.11). During early development (P5 and P10) there is a relatively uniform population of cells across the nuclei, indicated by the tight distributions about the mean values. As the animals mature, the distribution of cells becomes diverse. However, at P47, the data have not formed two distinct populations, and possibly indicate that values for cell areas have not yet stabilised.
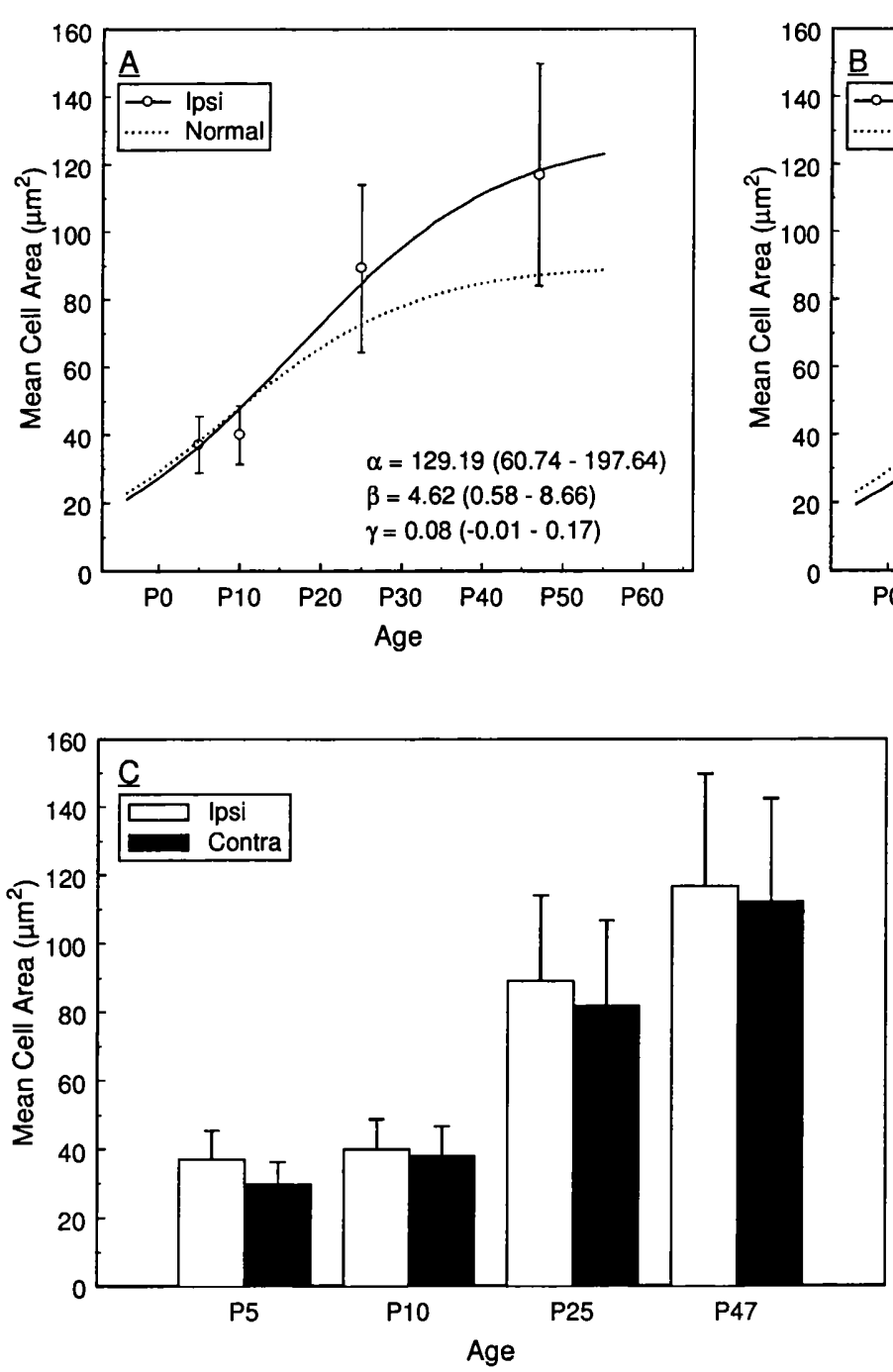

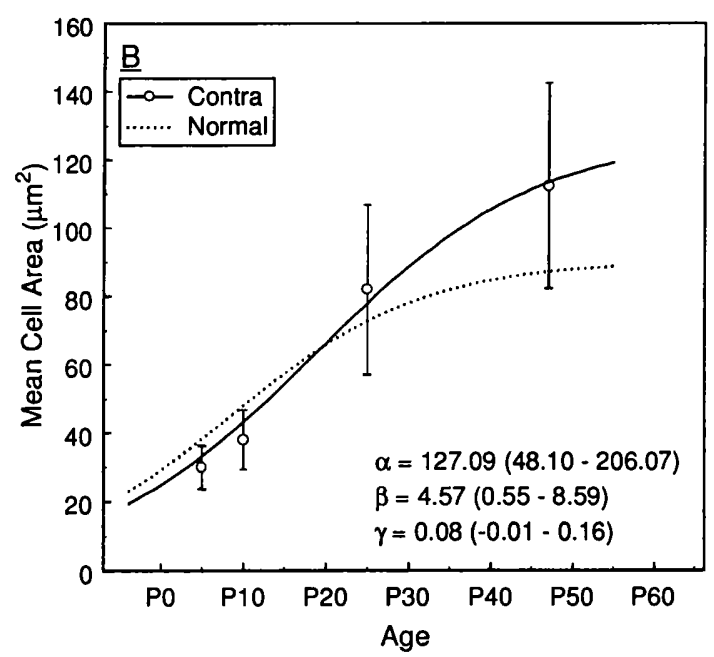

Figure 7.3 - Mean cell size in the LGN ipsilateral (A) and contralateral (B) to the enucleated eye. Measurements are of cell cross-sectional areas taken from one section mid-way between the dorsal and ventral poles of the nucleus at uniformly sampled locations (see Section 4.2.3). The solid lines are growth curves fitted to the data, and the dotted line

represents the growth curve fitted to the data from the normal animal (Chapter 4). (C) The same data is replotted as a bar chart for comparison between ipsilateral and contralateral nuclei. 

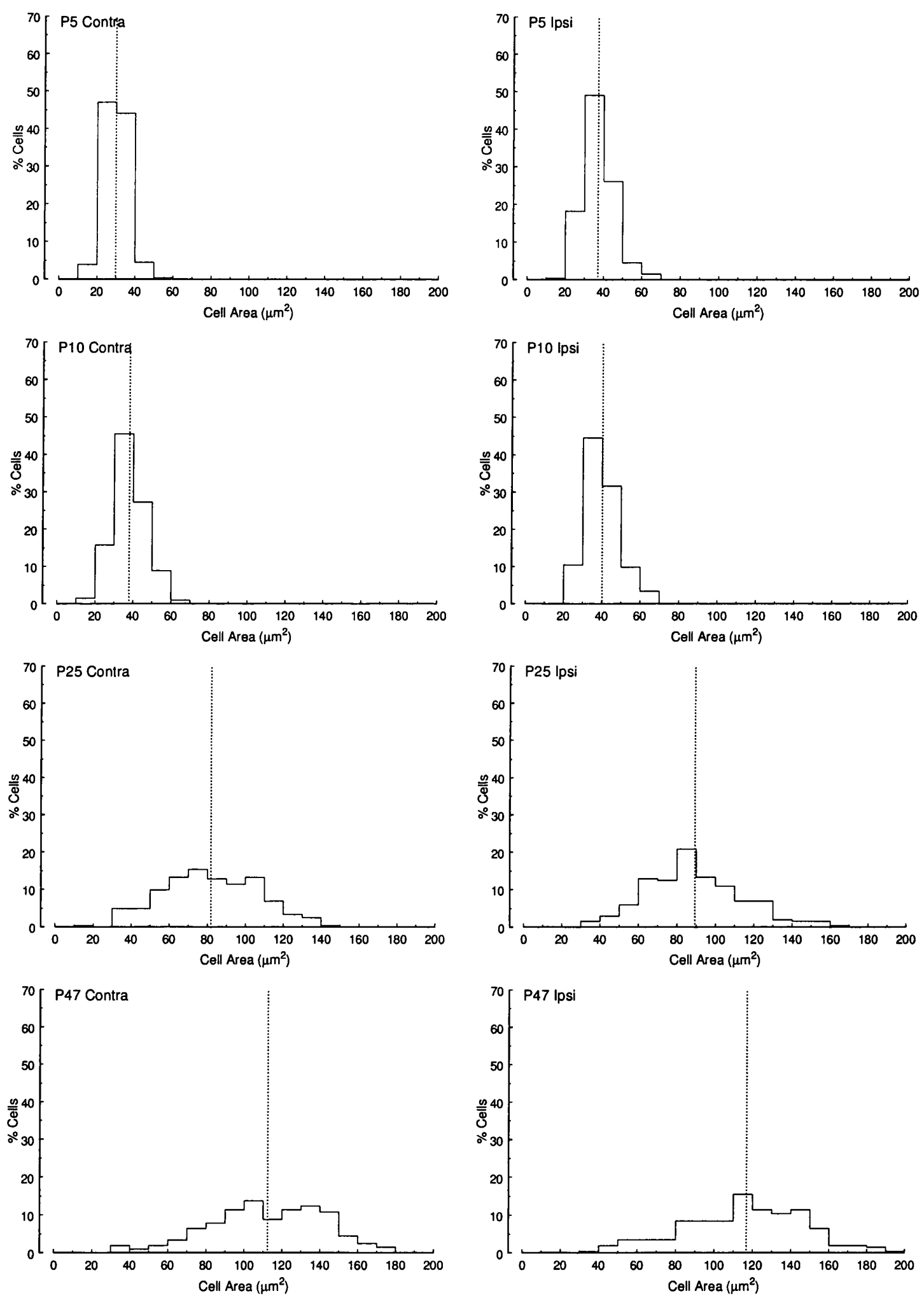

Figure 7.4 - Distributions of cell sizes as measured from cross-sectional areas taken from one section mid-way between the dorsal and ventral poles of the LGN ipsilateral (right column) and contralateral (left column) to an enucleated eye. The dotted lines indicate the mean cell area of each sample. The number of cells measured in each sample was 200 ( 2 animals, 100 cells each). 


\subsubsection{Contributors to LGN growth in the enucleate.}

Chapter 4 showed that the volume of space occupied by cell bodies within the LGN decreases with time, i.e. the ratio of LGN volume to the volume of cell bodies increases from approximately 5:1 early in development to approximately 15:1 in maturity. Given that total cell numbers in the enucleate are substantially different from the normal animal, as well as differences in volume (Section 7.3.2), and cellular size (Section 7.3.3), do the cellular constituents of the enucleate LGN contribute the same proportion to its growth?

Figure 7.5 shows the ratio of LGN volume to the volume of cell bodies within the nucleus ipsilateral and contralateral to the enucleated eye. The figure also shows the ratios calculated for the normal animal during development. At all ages analysed, the ratios calculated for the enucleated animal are consistently higher than the normal, although they are in broad agreement in that there is a general trend for the ratio to increase almost linearly with age.

During the first two postnatal weeks, LGN volume is similar to the normal, as is cellular size. However, cell numbers are lower in the nucleus ipsilateral to the enucleation, and lower still contralaterally. The resultant effect of this decrease in cell number is an increase in the ratio of LGN volume to the volume of cell bodies (Figure 7.5). At later stages of development, the ratios for the enucleated animal increase further due to the combined effects of reductions in LGN volume and lower cell numbers, despite a measured increase in cellular size. 


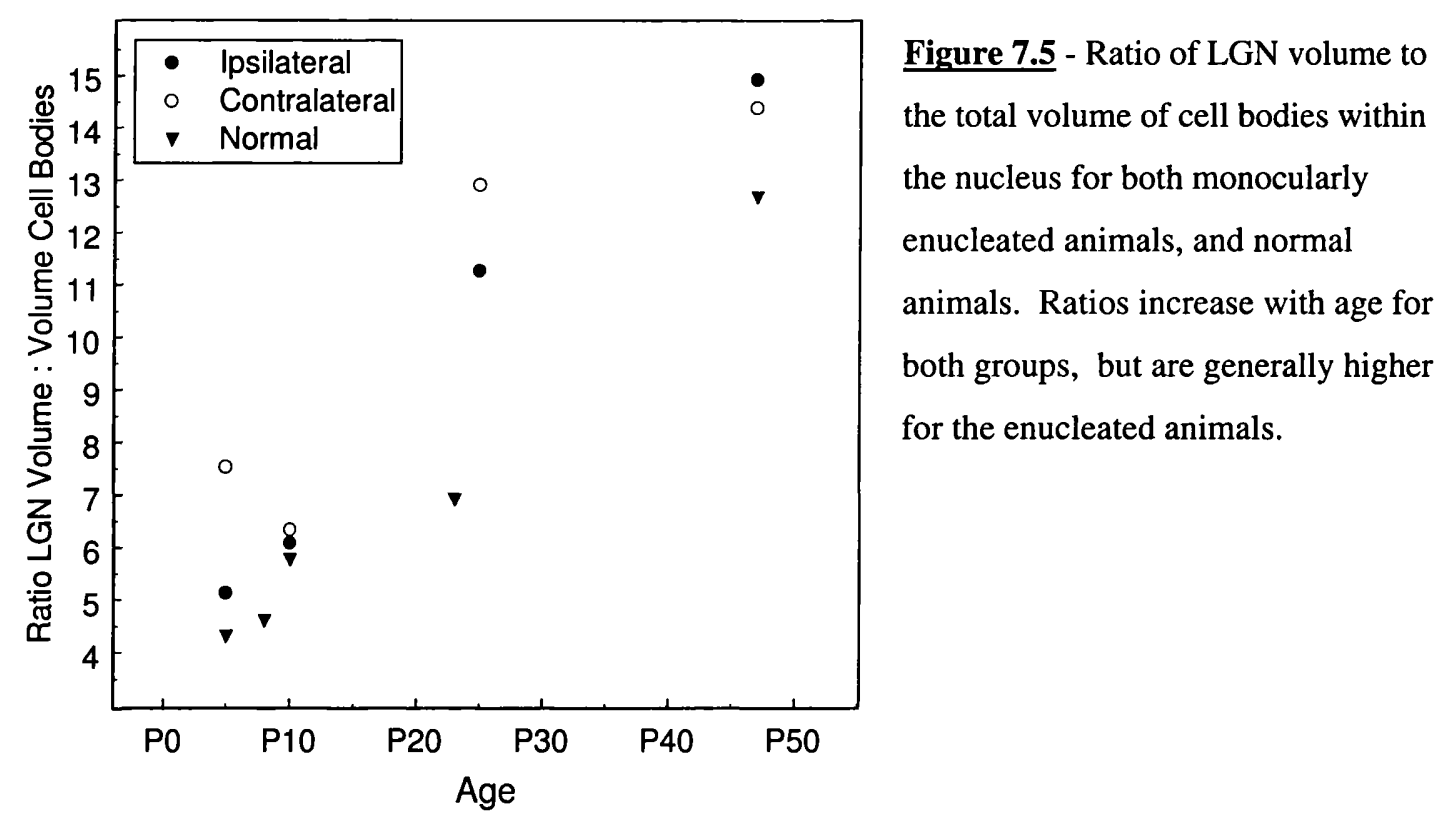

\subsubsection{LGN shape changes ipsilateral and contralateral to an} enucleation.

\subsubsection{General observations.}

The surface models of all individual geniculate nuclei from animals which were monocularly enucleated are shown in Figures 7.6a \& 7.6b (smaller diagrams). Averaging of the spherical harmonic coefficients describing these structures produced the mean models shown (larger diagrams). It is immediately evident from the diagrams, that the enucleated and normal animal have certain developmental changes in common, in particular the lateral rotation of the nucleus, and a substantial expansion of the dorso-ventral axis. There is a degree of similarity between ipsilateral and contralateral structures early in development (P5 and P10), in addition to a correspondence with the nuclei from the normal animal (compare with Figure 5.3). At P25 and P47, the nuclei ipsilateral to the enucleation still resemble the normal animal, whereas the contralateral nuclei have a generally thinner appearance. 


\section{Contralateral}

\&
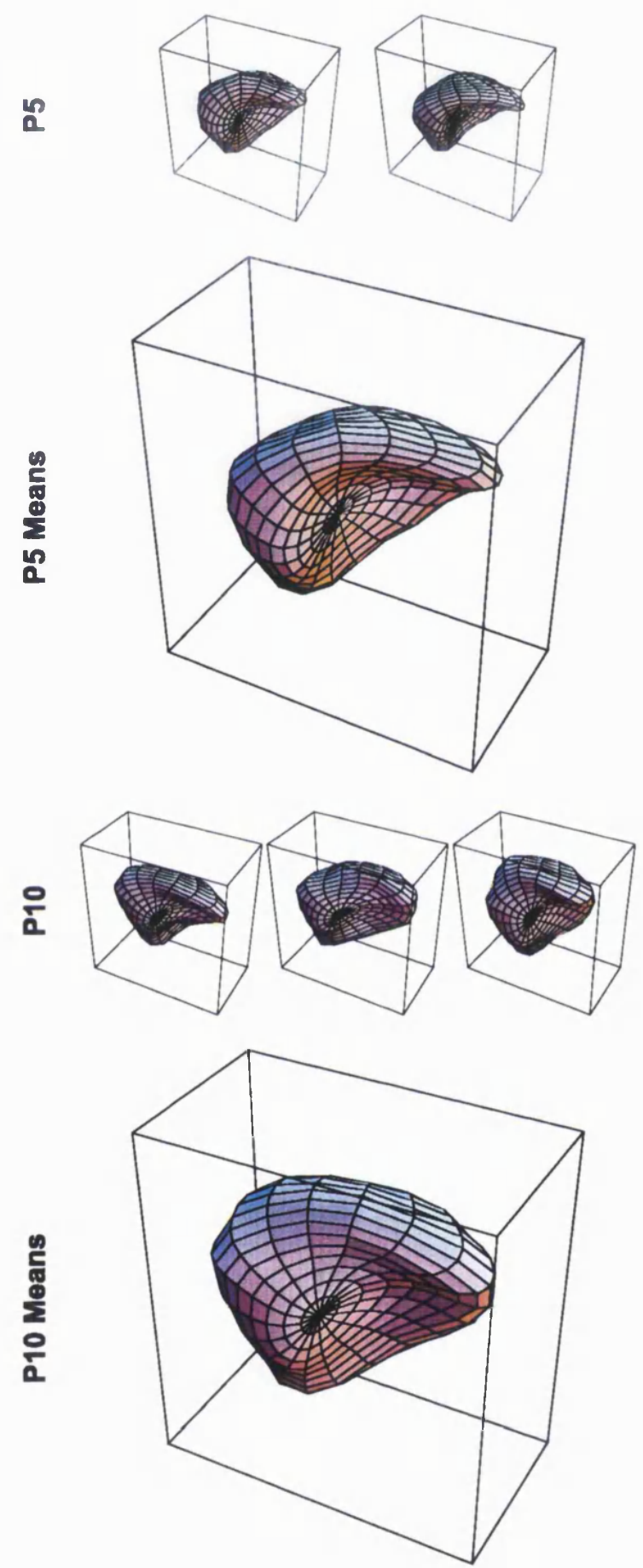

Ipsilateral
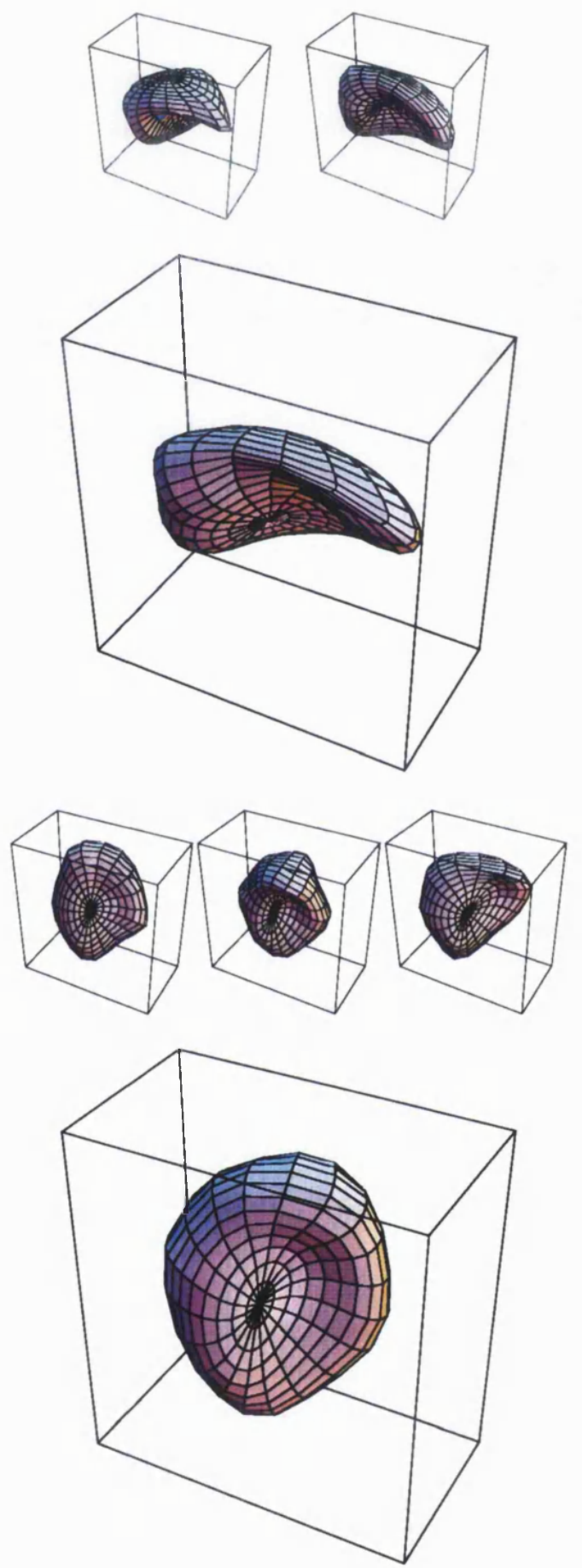

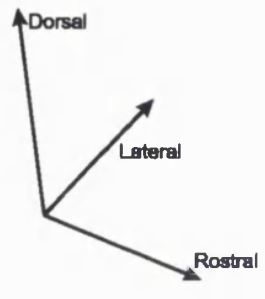

Figure 7.6a - Plots of individual and mean models of the LGN ipsilateral (right column) and contralateral (left column) to an enucleated eye at the ages indicated. Orientation is as shown on the left, and the scaie of the mean models is indicated on the

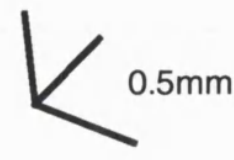
right. 
Contralateral

:
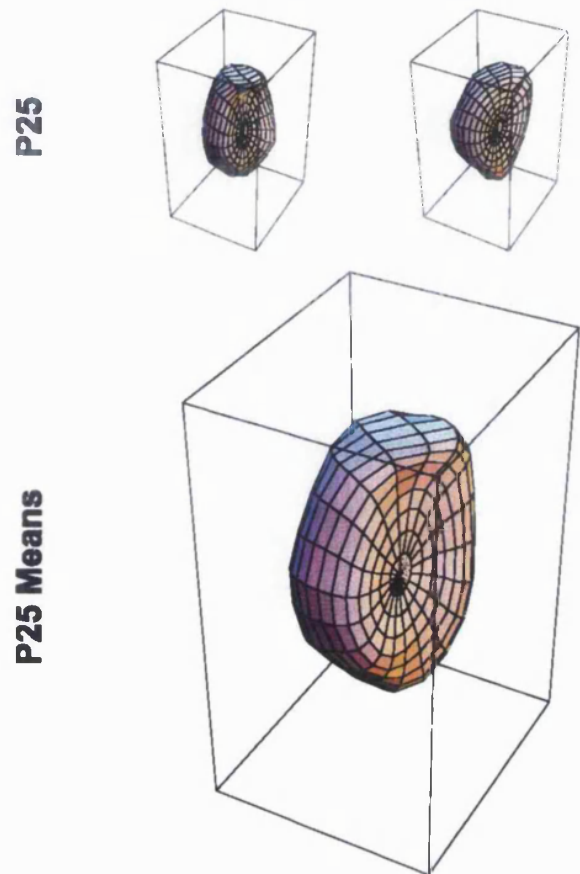

ษ
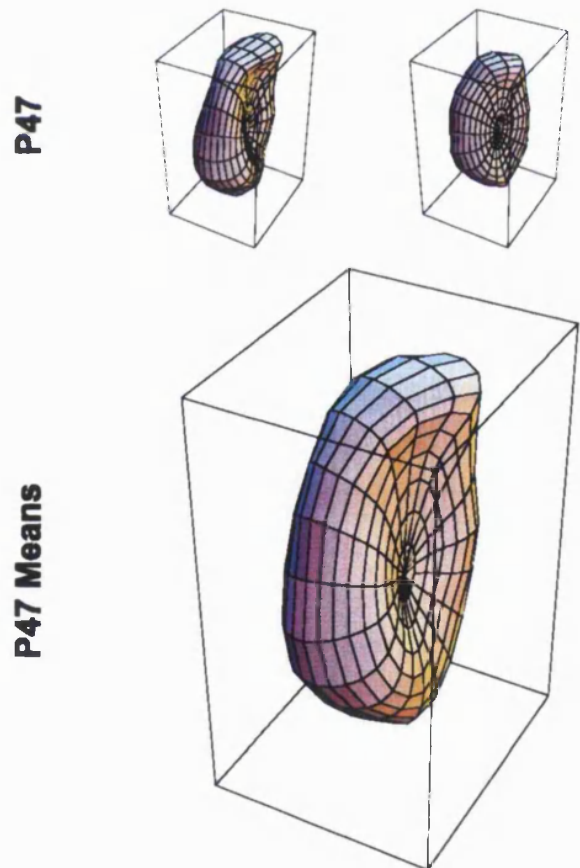

Ipsilateral
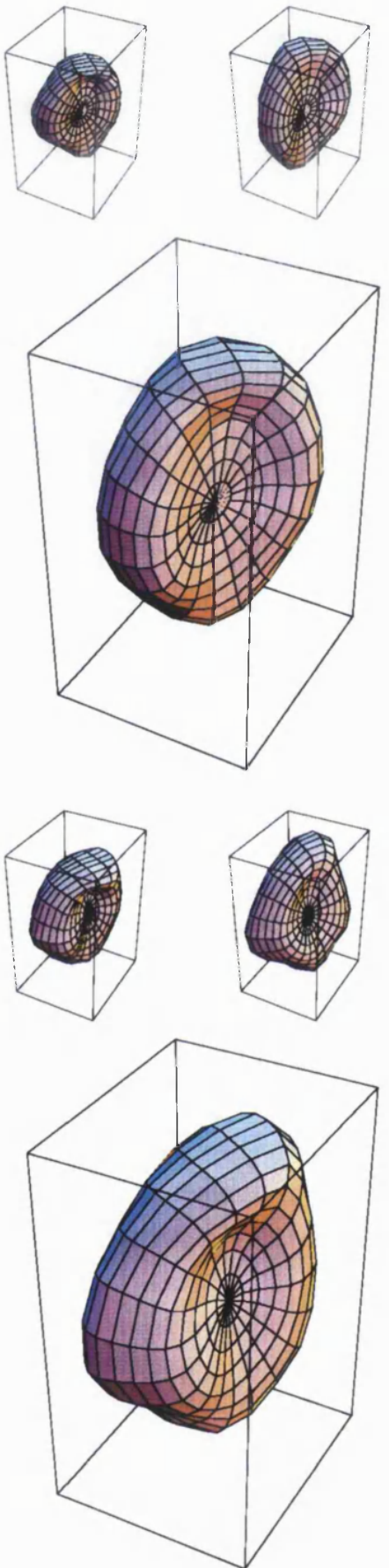

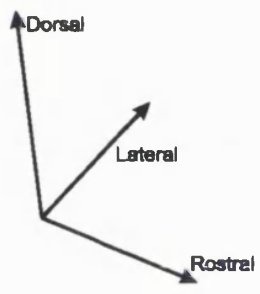

Figure 7.6b - Plots of individual and mean models of the LGN ipsilateral (right column) and contralateral (left column) to an enucleated eye at the ages indicated. Orientation is as shown on the left, and the scale of the mean models is indicated on the

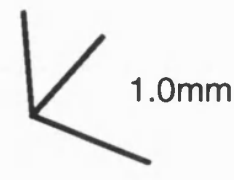
right. 
In the P5 animal, the axial dimensions of the nuclei ipsilateral and contralateral to the enucleation are practically the same as the normal. The only exception is a reduction in the rostro-caudal axis of the contralateral nucleus by approximately $25 \%$ (1.7mm in the normal, $1.3 \mathrm{~m}$ in the enucleate). By $\mathrm{P} 47$, this axis (which, due to the rotation of the nucleus, has become the medio-lateral axis) has expanded such that there is only a $10 \%$ difference between the normal $(1.8 \mathrm{~mm})$ and the enucleate $(1.6 \mathrm{~mm})$. In this same nucleus, the rostro-caudal axis is reduced by approximately $29 \%$ in comparison to the normal $(1.1: 1.6 \mathrm{~mm})$, and accounts for the generally thinner appearance of the nucleus.

Ipsilateral to the enucleation, the P47 animal remains similar to the normal in all dimensions, except for a small increase in the medio-lateral axis $(1.8 \mathrm{~mm}$, normal; $1.9 \mathrm{~mm}$, enucleate). This, together with the reduction in the same axis of the contralateral nucleus, equates to the $20 \%$ reduction previously reported between ipsilateral and contralateral nuclei in this dimension (Guillery et al., 1985). However, results here do not corroborate the $20 \%$ reduction that has been observed in the dorsoventral axis between nuclei ipsilateral and contralateral to the enucleation (Guillery et al., 1985).

In order to confirm that the nuclei ipsilateral to enucleation are more similar to the normal animal, the Euclidean distance was calculated between each mean model of the enucleated animal and its normal counterpart (Figure 7.7). In order that these dissimilarity indices are put into context, the mean Euclidean distance between the individual normal models and the mean normal model at each age is also provided. This may be considered as the mean variation in shape of the normal animal. In three of the four age groups (P5, P10, P47), the LGN ipsilateral to the enucleation is more 
similar to the normal animal. During development, the difference in Euclidean distance between the ipsilateral and contralateral nuclei is small, indicative of a similarity between these nuclei at these times. By adulthood (P47), the difference is substantially larger, and the Euclidean distance between the ipsilateral nucleus and the corresponding normal nucleus is much reduced, and is comparable with the variation seen between the individual normal models.

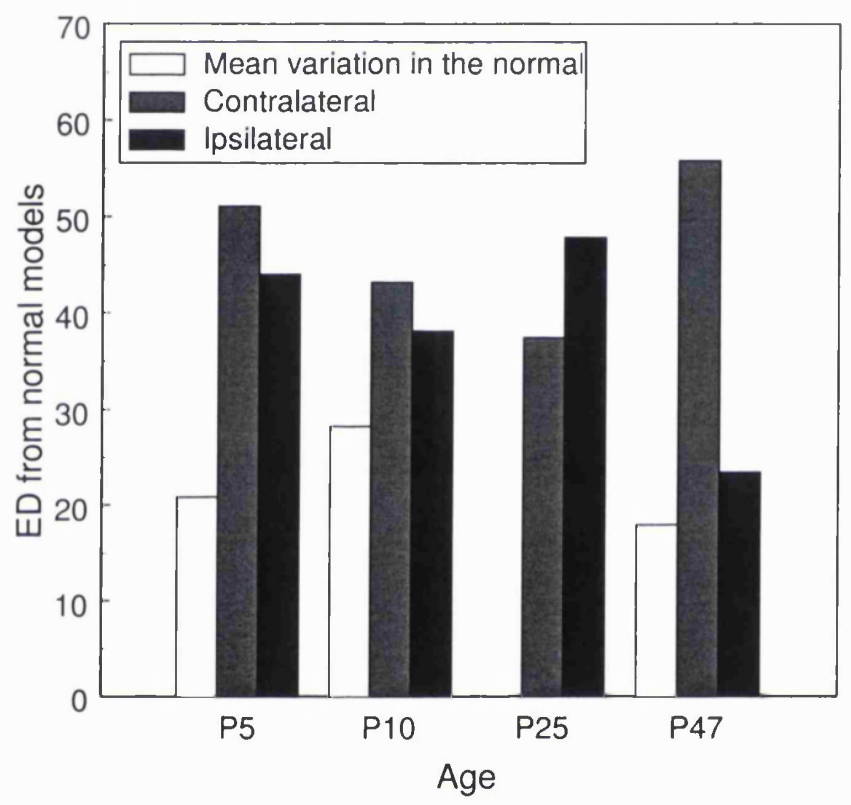

Figure 7.7 - A comparison of the similarities between nuclei of the enucleate and normal animals at successive stages of development. The bars show Euclidean distances (ED) between the mean contralateral/ipsilateral nuclei and their normal counterparts. The mean variation in shape of the normal animal is also shown. In three out of four cases, the nucleus ipsilateral to enucleation is more similar to the normal animal. By P47, the dissimilarity between the ipsilateral and normal nucleus is approximately the same as the mean normal variation.

\subsubsection{Temporal changes in shape.}

In a similar fashion to the normal animal, the temporal aspects of LGN shape changes ipsilateral and contralateral to an enucleation may be established by calculating the Euclidean distance between the E38 normal nucleus and every LGN modelled from the enucleates. The E38 normal nucleus is considered a valid base point since the 
enucleations were performed at $\mathrm{P} 0$, and also provides a common starting point for comparison with the normals.

Figure 7.8a shows the dissimilarity between the mean E38 normal LGN and every individual nucleus of the enucleate. While this shows the raw dissimilarity data, together with the fitted growth curves, the equivalent normalised curves (Figure 7.8b), together with the normalised curve from the normal animals provide a clearer picture of the temporal aspects of shape change. The shape of the curve describing structural change in the ipsilateral nucleus is very similar to that of the normal animal, but out of phase by approximately 5-7 days. This is also reflected in the fact that these two nuclei have a similar peak in the rate of shape change, but again displaced by approximately 5-7 days (Figure 7.8c). Contralateral to the enucleation, the LGN changes shape at a much higher rate, and reaches maturity during the early stages of the fourth postnatal week. 


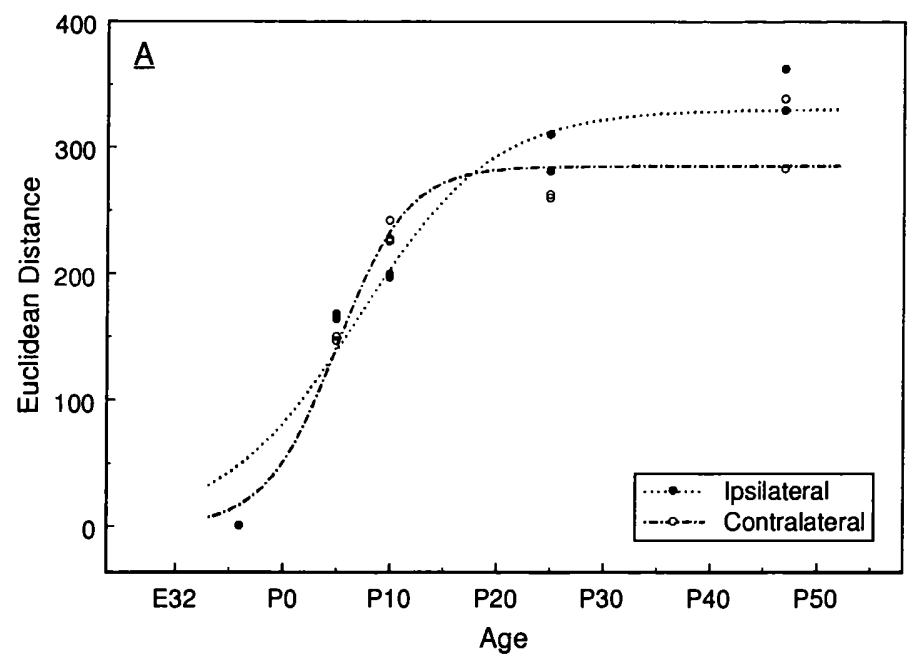

$$
\begin{aligned}
& \text { Ipsilateral } \\
& \alpha=330.06(287.53-372.59) \\
& \beta=7.79(2.88-12.70) \\
& \gamma=0.16(0.06-0.26)
\end{aligned}
$$

\section{Contralateral}

$\alpha=284.76(254.50-315.03)$

$\beta=14.34(5.25-23.44)$

$\gamma=0.31(0.12-0.49)$
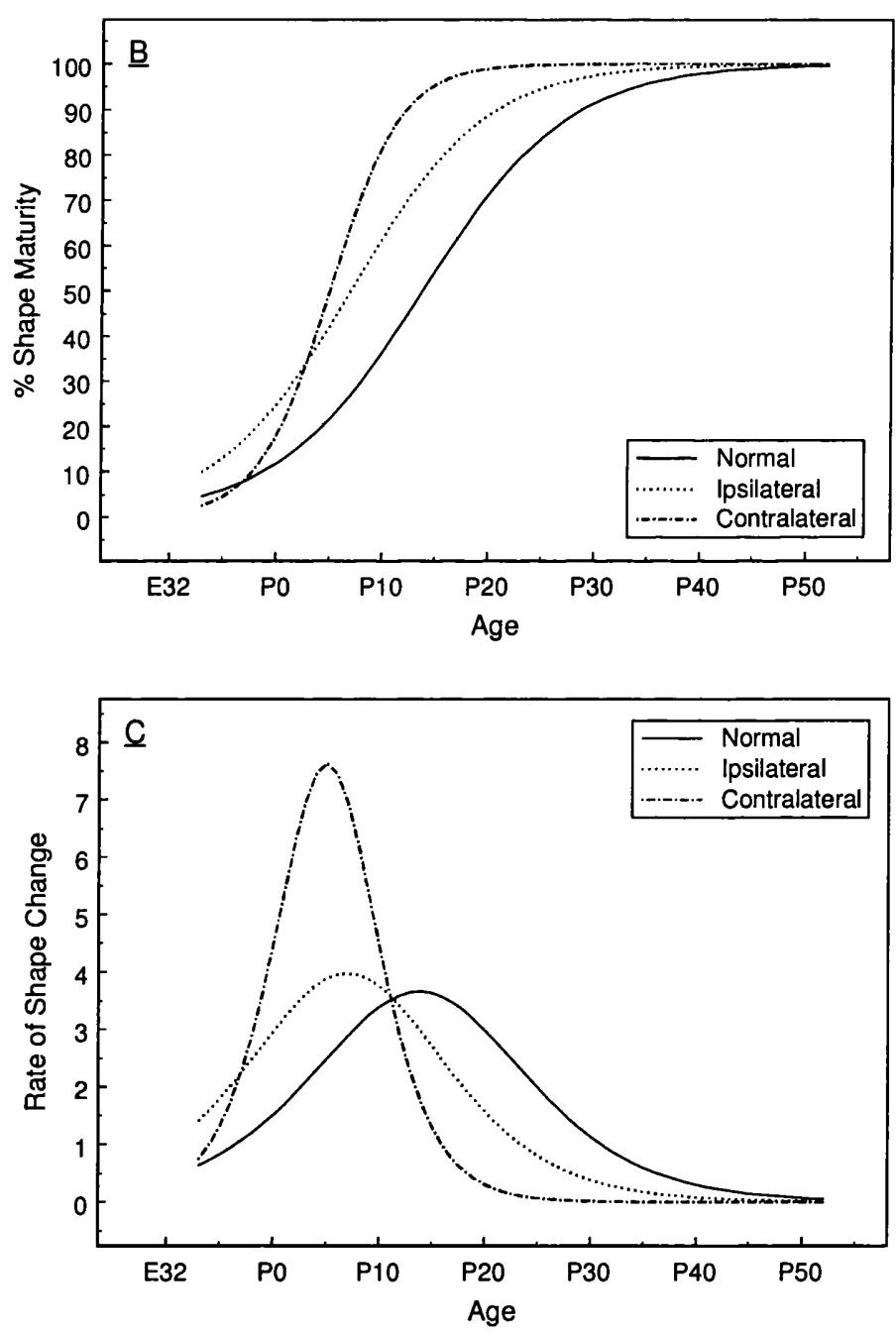

Figure 7.8 - (A) Euclidean distances between the mean E38 normal LGN and every nucleus modelled from the enucleates. The estimated parameters of the fitted growth curves are shown on the right together with their respective $95 \%$ confidence intervals. (B) The curves fitted to the data in $\underline{A}$ have been normalised to express shape change as a proportion of maturity, and the curve derived for normal development is also shown. $(\underline{C})$ The first differential of the curves in $\underline{B}$ provide information on the rate of change. 


\subsubsection{Specific local changes in shape.}

The specific changes in LGN shape between time periods for animals which have been monocularly enucleated on the day of birth are shown in Figures 7.9-7.12. Accompanying these figures are corresponding changes occurring in the normal animal. Unlike the figures shown in Chapter 5, which showed only those vectors greater than the mean magnitude, here all vectors are shown since it is the general patterns of change which are of interest.

Between E38 and P5, there is a substantial overall similarity in the pattern of growth of the normal nucleus and that of the nucleus ipsilateral to an enucleated eye (Figure 7.9). The only exception to this is the growth in a medial direction of the medial aspect of caudal LGN in the normal animal, which is not present at all in the ipsilateral nucleus. This represents the region into which the uncrossed projection would retract in the normal animal. Contralateral to the enucleated eye, the overall pattern of growth bears no similarity to the normal animal. Comparison of the data presented here with those in Figure 5.14 reveal that the changes occurring during this period are more consistent with those occurring between E38 and P0 in the normal animal. This suggests that the development of the nucleus is delayed or abnormal.

Figure 7.10 shows the growth of the LGN between P5 and P10. Here again, the growth of the nucleus ipsilateral to the enucleation resembles that of the normal animal. On the opposite side of the brain, the growth of the contralateral nucleus reveals a pattern more consistent with that shown between E38 and P5 (Figure 7.9), thus exemplifying further the notion that growth of the nucleus contralateral to the enucleation is delayed at this stage. 


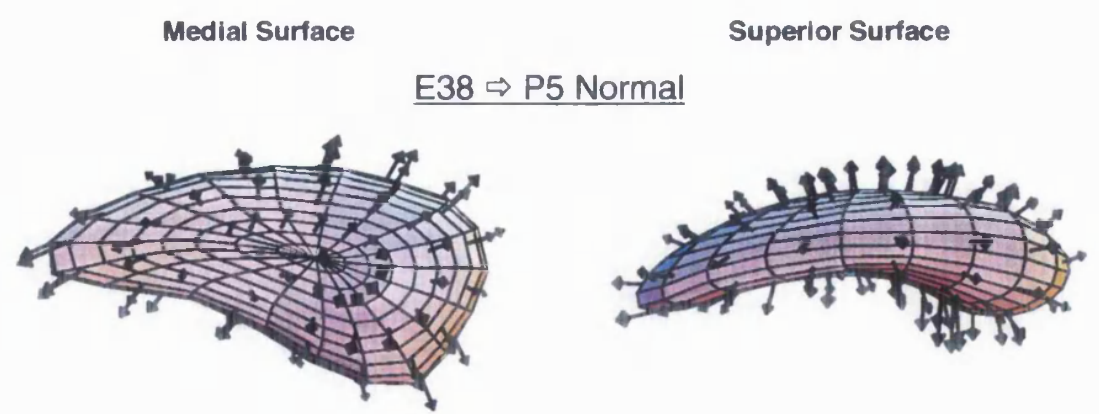

E38 $\Rightarrow$ P5 lpsilateral
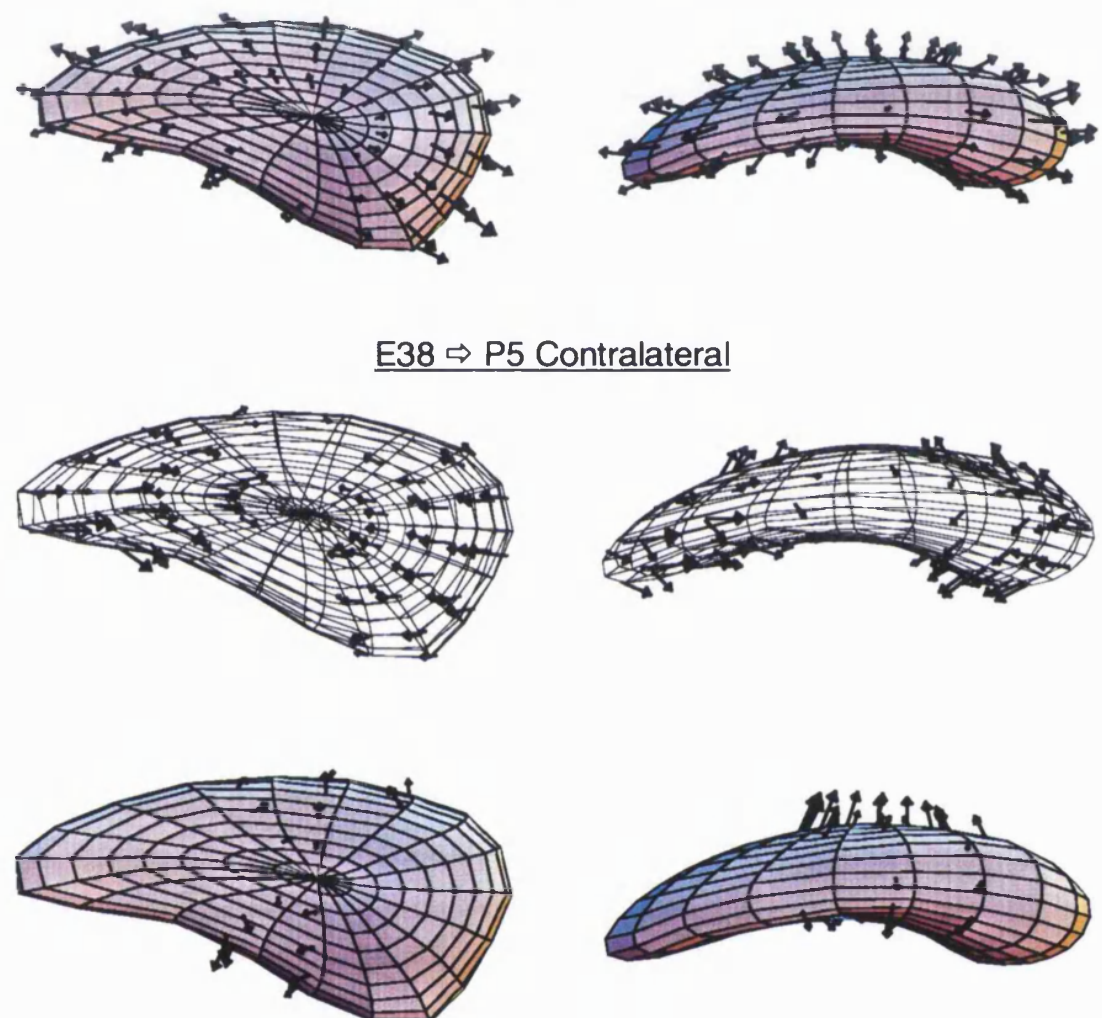

Figure 7.9 - Specific changes in shape between E38 and P5 nuclei from normal and monocularly enucleated animals. The nucleus shown is the mean E38 model, and the vectors show the changes in shape required to transform it to the respective mean P5 shapes. The left column shows the medial surface of the nucleus, and the right shows the superior surface. Rostral is towards the left. 


\section{$\underline{P} 5 \Rightarrow$ P10 Normal}
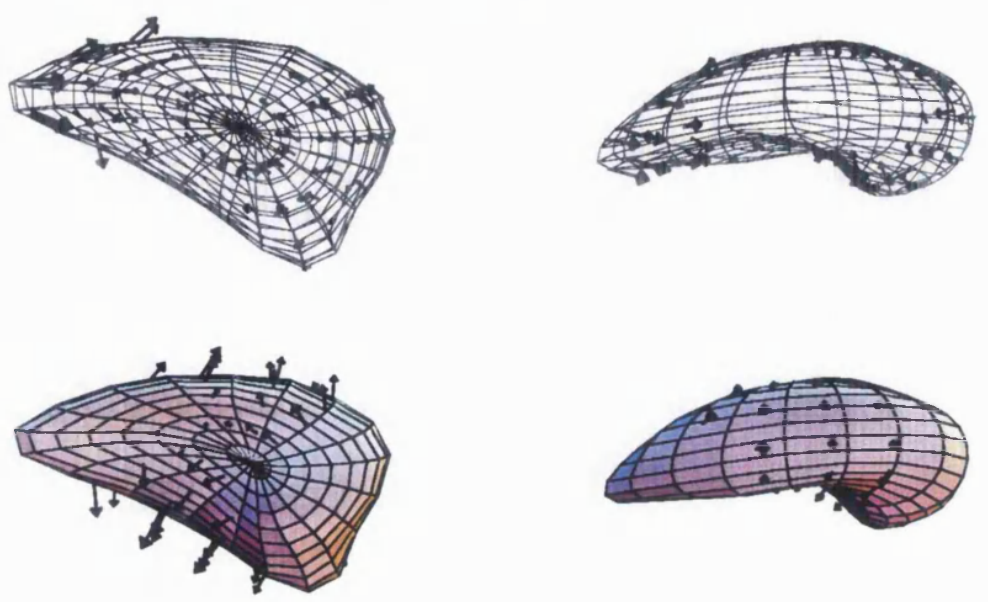

\section{$\underline{P 5} \Rightarrow P 10$ losilateral}
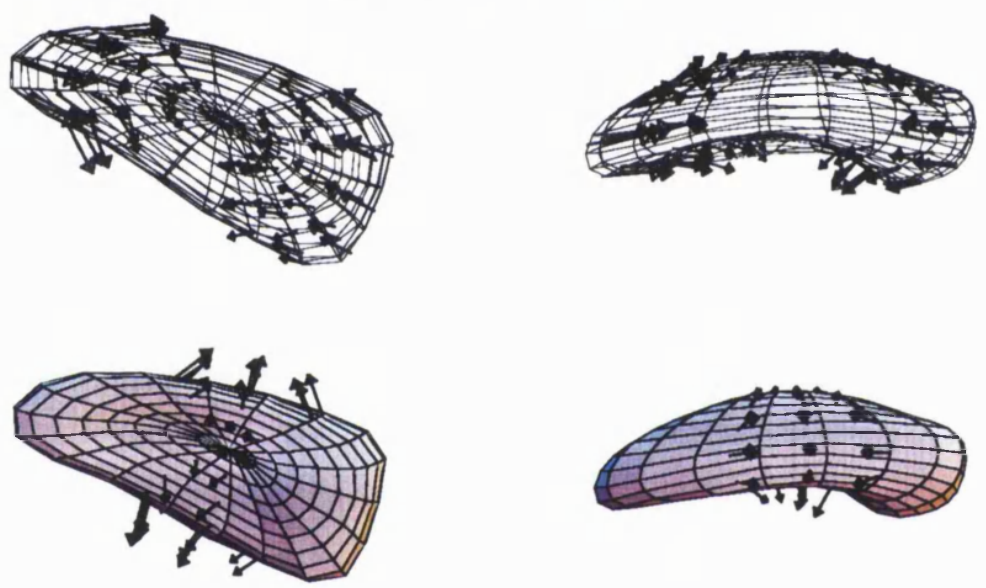

$\underline{P 5} \Rightarrow \mathrm{P} 10$ Contralateral
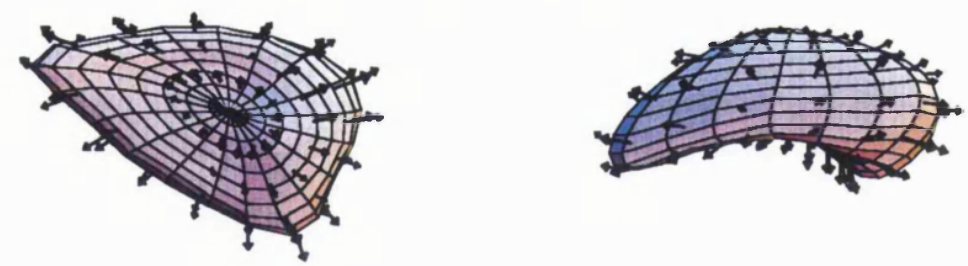

Figure 7.10 - Specific changes in shape between P5 and P10 nuclei from normal and monocularly enucleated animals. The nuclei shown are the mean P5 models, and the vectors show the changes in shape required to transform them to the respective mean P10 shapes. The left column shows the medial surface of the nucleus, and the right shows the superior surface. Rostral is towards the left. 
During the period between P10 and P25, growth of the nucleus ipsilateral and contralateral to the enucleation are similar. The mean vector lengths describing changes to the ipsilateral nucleus are more than twice those of the normal animal, and half as much as the changes between P25 and P47. This could possibly explain why this nucleus reaches maturity before the normal.

Figure 7.12 also shows some isotropic growth for the nucleus contralateral to the enucleation between P25 and P47. This is somewhat unexpected since Section 7.3.5.2 showed this nucleus to reach maturity during the fourth postnatal week (Figure 7.8b). Since the changes described in this section reflect real displacements of the surface of the nuclei, it must be assumed that the growth curve representing the development of the shape of the contralateral nucleus is not accurate, especially in terms of the time of maturity. This may be due to a number of factors, including the number of animals used in this study, but in particular the relative similarity of one of the P47 contralateral nuclei to a P25 nucleus, and the dependence of the curve fitting algorithm on the relatively low Euclidean distance of this nucleus.

While the above has shown the nature of specific changes to the enucleated animal, and compared them with changes occurring at corresponding times in the normal, Figure $7.8 \mathrm{~b}$ indicates that comparisons with the normal animal may be more appropriate with different ages. For example, it has been shown that the development of the LGN ipsilateral to the enucleation is advanced by approximately 5-7 days. It may therefore be more appropriate to compare the changes between the E38 and P5 nuclei with those of the P1 and P10 nuclei of the normal animal. 
It has only been possible to perform a small number of these comparisons, since models of the normal nucleus at relevant ages were not available. Changes between the E38 and P5 ipsilateral nucleus have been compared with the P0 and P10 normal nuclei, and the vector diagrams are in broad agreement (figure not shown). Similarly, the changes between the E38 and P5 contralateral nucleus have been compared with the E38 and P0 normals (Figure 5.14), and as was stated earlier, these are also in agreement.

According to Figure 7.8b, changes between the P5 and P10 contralateral nuclei, should be likened to those of the P12 to P24 normal nuclei. These ages were not available, and comparisons have therefore been made with the P10 and P23 normals (Figure 5.17). Once again these figures are similar in showing an isotropic growth of the LGN in both normal and enucleated animals.

The final comparison which has been possible is for the changes occurring between the P25 and the P47 ipsilateral nucleus. This age range should be compared with the P30 and P52 nuclei of the normal animal, but the closest comparison which has been possible is the changes occurring between the P36 and the adult nucleus (Figure 5.19). The changes in the normal animal are very small during this period, and this is also the case for the enucleated animal. 

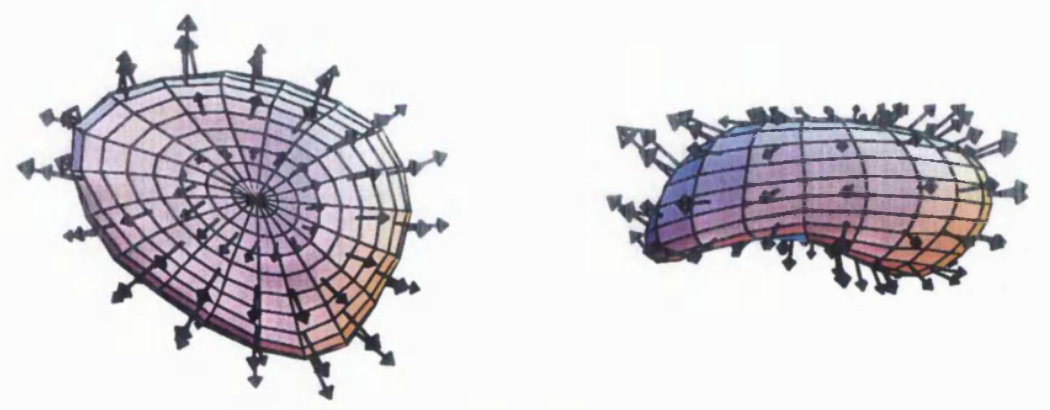

\section{P10 $\Rightarrow$ P25 Ipsilateral}
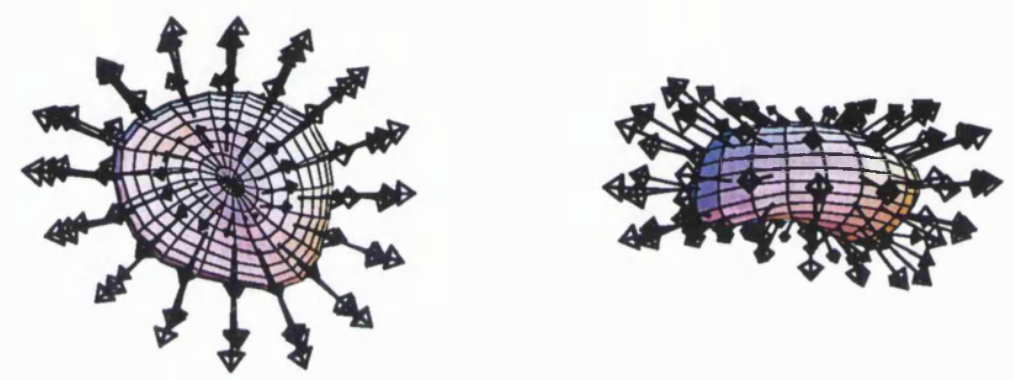

\section{P10 $\Rightarrow$ P25 Contralateral}
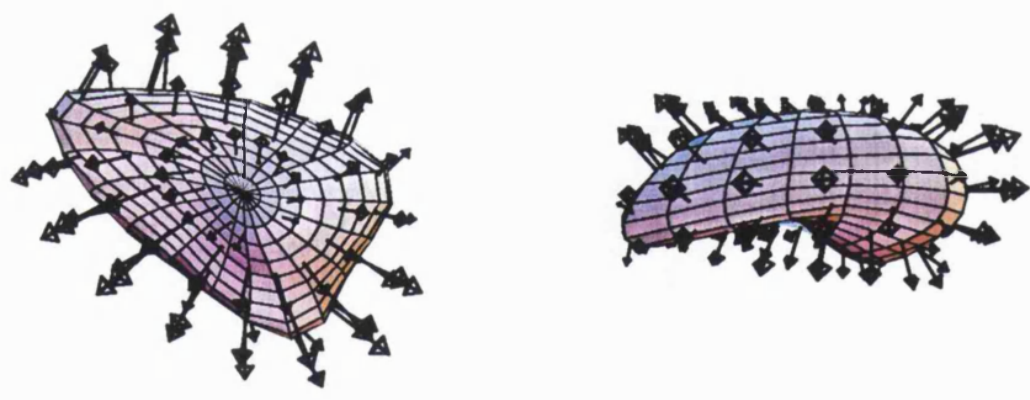

Figure 7.11 - Specific changes in shape between P10 and P23 nuclei from normal animals, between P10 and P25 monocularly enucleated animals. The nuclei shown are the mean P10 models, and the vectors show the changes in shape required to transform them to the respective mean P23/25 shapes. The left column shows the medial surface of the nucleus, and the right shows the superior surface. Rostral is towards the left. 

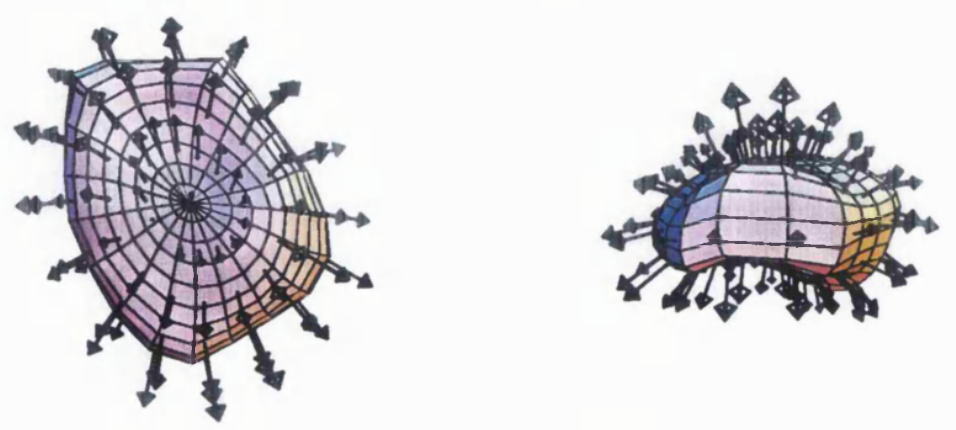

P25 $\Rightarrow$ P47 lpsilateral
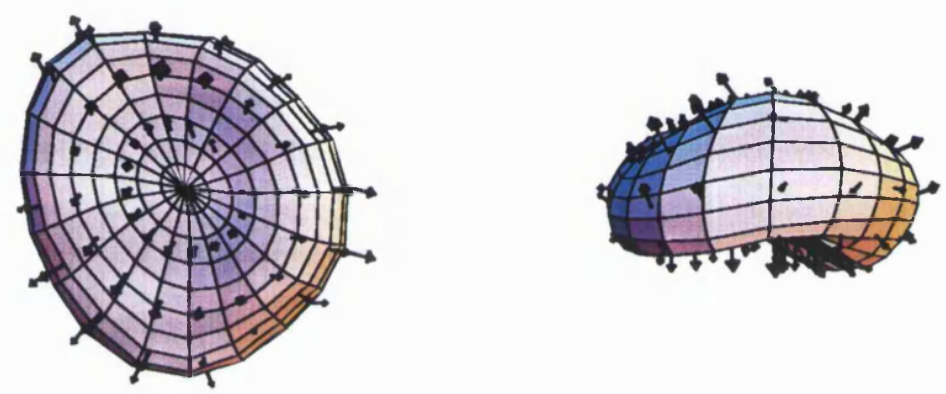

P25 $\Rightarrow$ P47 Contralateral
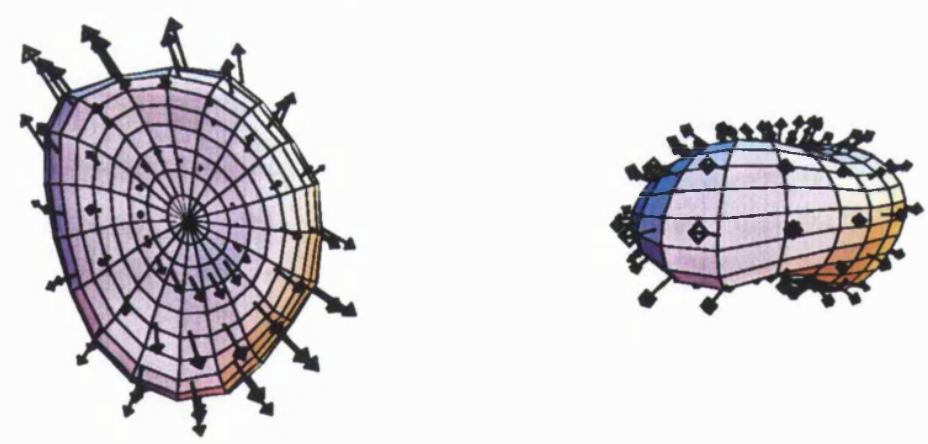

Figure 7.12 - Specific changes in shape between P23 and P47 nuclei from normal animals, between P25 and P47 monocularly enucleated animals. The nuclei shown are the mean P23/25 models, and the vectors show the changes in shape required to transform them to the respective mean P47 shapes. The left column shows the medial surface of the nucleus, and the right shows the superior surface. Rostral is towards the left. 


\subsubsection{Temporal sequences of events - comparisons with the normal animal.}

Figure 7.13 shows the maturation of the LGN ipsilateral and contralateral to the enucleated eye in terms of nuclear volume, cellular density, cellular size, and change in shape. Maturation is measured as a percentage of the adult value. Similar data for the normal animal are also shown for comparison (taken from Figures 5.11 \& 5.12). Ipsilateral to the enucleated eye, the sequence of events is the same as for the normal animal, but the timing of events is different. The shape of the nucleus attains full maturity at approximately $\mathrm{P} 40,5$ days prior to the normal animal as was stated in Section 7.3.4.2. This is closely followed by a stabilisation in the density of cells within the nucleus at P45. Finally, LGN cell sizes, and the volume of the entire nucleus reach maturity between P50 and P60. Given that there is a degree of error in the parameter estimates of the respective growth curves, it may be the case that this difference in the timing of events is not real. However, whether this is truly the case cannot be ascertained, and more data would be required to construct a more accurate growth curve.

A similar picture is evident for the nucleus contralateral to the enucleated eye: the sequence of events is the same as for the normal animal, but again, the timing of events is different. In particular, the time at which shape maturity is reached (during the beginning of the fourth postnatal week) is substantially earlier than that of the normal animal, and the rate at which it matures more than doubles. Once more however, the degree of error in the growth curve parameters could be responsible for this phenomena. 
Peaks in rates of maturation occur at similar times for nuclei in the enucleated animal, and are generally earlier than observed in the normal animal. The single exception is the rate of cell size maturation, which peaks approximately a week after the normal animal. However, it was stated earlier that the mean cell size in maturity could be lower than that measured here. If this is the case, then the peak in the rate of cell size maturation would be brought forward, and could therefore occur at a similar time to the normal. The confidence intervals for the growth curve parameter describing adult cell sizes are extremely large due to the substantial standard deviations prevelant in the original data, but indicate that mean cell sizes may well be lower.

\subsection{Discussion.}

The consequences of performing a monocular enucleation on the day of birth have been explored during development with respect to changing LGN shape, and aspects of its cellular constituents. In comparison with the normal un-operated animal, varying degrees of abnormalities have been measured in the enucleated animal. This is expected since the removal of one eye results in a substantial elimination of fibres innervating the nucleus contralateral to the enucleation, while only eliminating a very small amount innervating the ipsilateral nucleus.

\subsubsection{Cellular constituents of the enucleated animal.}

The LGN ipsilateral to the enucleated eye is relatively normal in terms of a number of parameters. The mean volume of the nucleus throughout development is similar to the normal animal, as is the mean density of cells within the nucleus. The total number of cells however is reduced by approximately $20 \%$. Contralaterally, the volume of the nucleus is reduced in the mature animal, as has been previously 

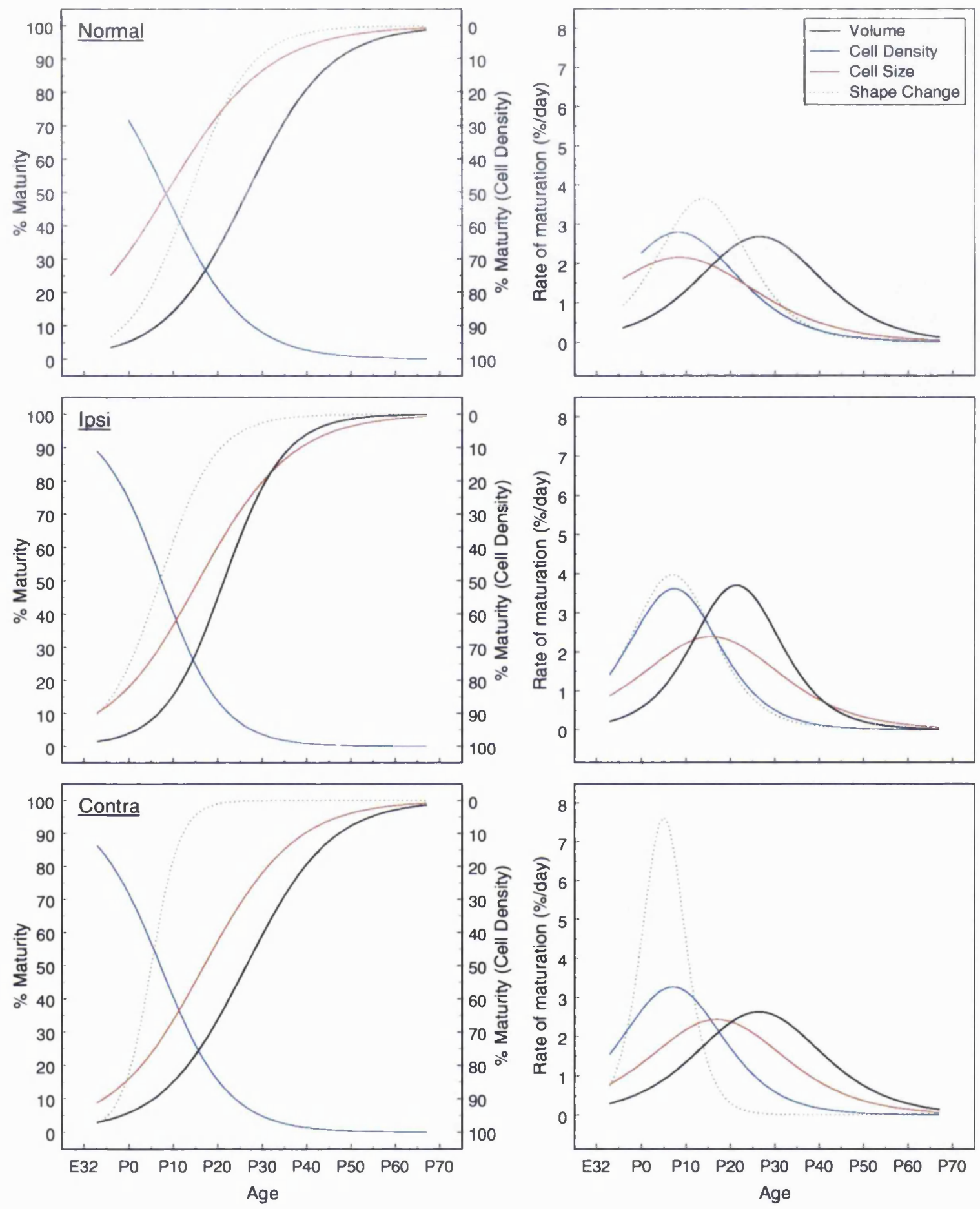

Figure 7.13 - Maturation of the LGN in terms of nuclear volume, cell density within the nucleus, cell growth, and changes in shape. The figures on the left show the temporal pattern of maturation, while those on the right show the rate of maturation. All parameters of the enucleated animals reach their adult values in the same sequence as shown for the normal animal. 
reported (Thompson et al., 1993). Similarly, the total number of cells is reduced more than the ipsilateral nucleus (approximately $46 \%$ from the normal), and this is also as expected (Finlay and Pallas, 1989). Cellular densities within the developing LGN of a monocularly enucleated animal have not been previously reported, although one study has measured cell density during development in the binocularly enucleated tree shrew (Brunso-Bechtold and Vinsant, 1990). The findings are consistent with those reported here in that there is a similarity between normal and experimental animals.

A surprising result (in comparison to the normal) is the increase in cell size both ipsilateral and contralateral to the enucleated eye towards the end of the fourth postnatal week, which continues until maturity. It was shown in the normal animal (Chapter 4) that cell size is reduced in adulthood, at a time which is not covered in this chapter. It may therefore be the case that cell sizes are also reduced at a later stage in the enucleate. This would fit with the results of Kalil (1980) who has shown that in the monocularly enucleated cat there is no difference between cells of the enucleated animal and those of the normal animal. Brunso-Bechtold (1990) has also shown no significant difference in the size of cells between normal and binocularly enucleated tree shrews.

The proportion of LGN volume which is made up of cell bodies is lower for the enucleates than the normal animals, although there is a comparative trend with age between the groups. The ratio of LGN volume to volume of cell bodies is no doubt higher in the enucleates due to the reduced cell numbers, but also possibly due to the removal of extensive RGC axon arbors. The relative similarity between the ratios for nuclei ipsilateral and contralateral to the enucleation are due to a balance in the reduction of their respective volumes and cell numbers. In addition, the relative 
normality of the dendritic development of LGN neurones (Sutton and BrunsoBechtold, 1993), and the preserved corticogeniculate projection probably ensure that the ratios of volumes increase with time.

The results presented here indicate that there are a variety of consequences to monocular enucleations. The first type produce fairly acute changes to the cellular population throughout the developmental period investigated, exemplified by reductions in the total number of LGN cells. Other consequences of enucleations appear only to affect the animal towards maturity, such as the change in LGN volume. Regardless of these induced abnormalities, the contribution made by the cellular population of the LGN to its growth follows a similar pattern to that in the normal animal.

\subsubsection{Structure of the LGN.}

The nucleus ipsilateral to the enucleated eye develops normally in terms of shape, and the mature nucleus resembles that of the normal animal. Contralaterally, gross general changes to the nucleus can be observed during development, but in maturity the nucleus resembles the P25 ipsilateral nucleus in shape, with a minor increase in volume.

Does retinal innervation therefore contribute to the changes in LGN shape? The results presented here suggest that removal of a very small proportion of axons innervating the LGN result in an advancement in the maturation of its shape. In addition, removal of a more substantial proportion of the fibres innervating the nucleus advances the maturation even further. It thus seems that retinal afferents do not influence shape per se, but do have an effect on the temporal nature of shape changes, 
and possibly arrest the development early as is the case for the nucleus contralateral to the enucleated eye.

Experiments which have removed all retinal afferents (binocular enucleations) in ferrets on the day of birth (Guillery et al., 1985) further support this notion. The shape of the mature nucleus, although slightly abnormal, resembles the normal animal in that it has a broader thicker region medio-caudally (equivalent to the binocular region in the normal), which tapers to a thin elongated region further rostrolaterally. Also, the nucleus of the binocularly enucleated animal reaches maturity before that of the normal.

If the timing of the manipulations is brought forward, the results are more striking. In fetal ferrets which have been monocularly enucleated at E28, the mature nucleus contralateral to the enucleation is reduced in size compared to the same nucleus from an animal enucleated at birth (Guillery, 1989), and similar results are evident in congenitally anophthalmic animals (Cullen and Kaiserman-Abramof, 1976). The cytoarchitectural appearance of nuclei in these animals is not as clearly defined, and it can be difficult to interpret whether there are profound changes in shape present. Nevertheless, these studies indicate that if RGC axons are completely restricted from innervating the LGN, substantially greater abnormalities of the nucleus become evident. Similarly, if the visual cortex is removed, the LGN is substantially reduced in size, and also incurs substantial morphological abnormalities (Payne and Lomber, 1996; Boire et al., 1997). 


\section{Summary \& Conclusions.}

\subsection{The work in context.}

The work presented here has explored a novel hypothesis concerning the formation of eye-specific layers within the LGN of the ferret. Previous theories on ocular segregation have focused mainly on the role of activity-driven competitive interactions between ganglion cell axons (e.g. Shatz, 1994), but here the role played by differential growth of the nucleus has been investigated. Competitive interactions alone "cannot be the whole story since the (ocularly segregated) layers always form in the same pattern" (Shatz, 1996). It may be at this level that LGN structure and growth has a role to play in segregation, especially since ocularly distinct layers always form in the same way within each species, as does the LGN. But between species, there is variety in terms of LGN structure, which is accompanied by different patterns of lamination.

Two sets of experiments have led to this work: the first showed that there was retinotopic order present within the ferret LGN at birth (Jeffery, 1985). To bring about the normal pattern of binocular segregation seen in the mature animal, the maps from each eye need to shift relative to each other and the borders of the nucleus (Jeffery, 1989; 1990). Secondly, while the volume of the rodent LGN increases during 
the period of ocular segregation, the volume of the terminal field of ipsilaterally projecting fibres remains constant (Jeffery, 1984). These, taken together with the observation that there are substantial changes to the three-dimensional form of the nucleus during this period, suggest that the structural dynamics of the LGN could play a role in the process of segregation.

\subsection{Summary \& Conclusions.}

Prior to investigating changes in LGN shape, the size of the ipsilateral terminal field in the developing ferret was measured, and shown to remain constant, as reported for the rat (Jeffery, 1984). There were no statistically significant changes in the volume of the ipsilateral terminal field between $\mathrm{P} 0$ and $\mathrm{P} 12$, the period spanning the process of ocular segregation. The opposite is true for the volume of the entire nucleus during the same period. Note however that animal numbers within each group in this case were low, and may therefore have some influence on the significance of this statement.

\subsubsection{What contributes to the shape of the LGN?}

Changes to the LGN can occur as a result of intrinsic or extrinsic factors, or a combination of both. The work presented here has focused on the intrinsic factors, in particular the alterations to the constituent cellular population of the LGN. Surprisingly, the cells of the LGN (i.e. cell bodies) contribute very little to the substantial expansion in total volume that has been measured. While cell bodies occupy $20 \%$ of the nucleus during early development, this is substantially reduced to approximately $7 \%$ by adulthood. It is proposed that the remaining space is made up by dendritic trees, RGC and corticogeniculate cell axon arbors, and the late proliferation 
of glial cells. There is no evidence to suggest that the spatial distribution of cells within the nucleus, either in terms of density or size, contributes to the structure of the LGN, although this latter observation is based on measurements made in single horizontal sections only.

In order to provide baseline data to incorporate into other analyses, the shape of the LGN was quantified at successive developmental stages between E38 and P140. Considerable advances have been made recently in quantifying shape, and analysing changes in shape, although much of the work has been applied to landmark based data, or two-dimensional outlines (e.g. Rohlf and Bookstein, 1990; Marcus et al., 1996). Landmark based analyses of shape change were not appropriate here since the LGN has no identifiable landmarks at successive stages of development. Additionally, the LGN is a complex three-dimensional structure which is not adequately sampled by two-dimensional sections (i.e. outlines). The method employed here to characterise three-dimensional shape has only been recently proposed (Brechbühler et al., 1995). Not only does it provide an accurate visual representation of the surface of the LGN, but expresses it quantitatively using a relatively small number of parameters. This quantification has allowed the somewhat abstract quantity of 'shape' to be incorporated into analyses which include numerous anatomical measurements.

Several models of LGN structure were generated for a number of developmental stages, and these facilitated the calculation of the mean shape of the LGN at those stages. On a temporal scale, LGN shape assumes its adult state prior to any other aspect of the cellular population of the nucleus. It is unlikely that cell size and density contribute much to shape maturity, since the point at which these parameters have realised half their adult values, the shape of the LGN is less than a quarter mature. 
This is due to the fact that the temporal progression of these variables in not linear. The volume of the LGN appears to contribute more to structural development, but volume is a parameter which comprises a number of other elements. Results have shown that cellular bodies contribute little to volume (consistent with the fact that cell size and density contribute little to shape), and that dendritic trees and arbors of innervating axons contribute more. These should therefore play a greater role in the development of shape.

This conclusion has been confirmed in experiments which have employed monocularly enucleated animals. When the procedure is carried out on the day of birth abnormal structures are seen in the mature animal contralateral to the ablation, probably due to the reduced input. Consistent with this is a reduction in the volume of the nucleus. However, the impact of this type of manipulation is mild, and the result of this is that the shape of the nucleus, in general terms, appears to develop normally, albeit at a higher rate. It is considered abnormal since it matures at a point that would be considered immature in the normal animal. Following more severe manipulations such as binocular enucleations or cortical ablations, the volume of the nucleus is substantially lower (Guillery et al., 1985; Boire et al., 1997). Under these conditions, it is envisaged that the shape of the LGN would be severely affected.

Recently, it has become possible to protect retinal ganglion cells from naturally occurring cell death, resulting in more cells projecting to the LGN (Martinou et al., 1994). It would be of considerable interest to examine the geniculate under such conditions as more information would be gained as to how the input to the nucleus relates to its structure. Results here suggest that the nucleus would be larger in terms of volume, but it is difficult to envisage what changes in shape would take place. 
Would the LGN reach maturity at the same stage as the normal animal, and then grow proportionately; would the temporal aspects of shape change be advanced or delayed? Answers to questions such as these would provide greater insight into the relationship between RGC innervation and LGN structure than that provided here.

\subsubsection{Does shape change contribute to ocular segregation?}

An analysis of the specific spatial changes occurring between nuclei at different ages reveals a pattern consistent with the notions regarding binocular segregation and shape change. During specific temporal windows, there is an expansion in the rostrocaudal axis of the LGN. These changes could be envisaged as contributing to the shifting of retinotopic maps which bring about the adult pattern of binocular segregation as proposed by Jeffery $(1989 ; 1990)$. In addition, this pattern of change is consistent with the notion proposed in Chapter 3, that the ipsilateral projection moves into the caudal pole of the nucleus, while the contralateral projection moves from the same region.

However, there are other changes during different temporal periods (but within the period of ocular segregation) which do not fit with the differential growth hypothesis. These can be explained by the fact that comparisons between nuclei were made by centering them on their geometric centres of gravity, which may not be appropriate, and could contribute to errors. Future work should incorporate into the models the position of the LGN within the brain. It would then be possible to determine the spatial relationships between models at different ages, and whether it would be more appropriate to align models according to different criteria. In spite of this, during the entire period of ocular segregation there are distinctive patterns to LGN shape changes. This contrasts with the relatively isotropic pattern of growth 
which is found later, implying that there may be a role, possibly in ocular segregation, for the differential changes seen in early development.

Inevitably, the above evidence is entirely circumstantial, and does not show a direct relationship between shape change and ocular segregation. An attempt to find such a relationship was made by comparing the changes in nuclear shape to those of the terminal fields. Two approaches have been investigated, but neither have been successful. The first quantified the global transformations in LGN shape, and evaluated whether the same changes were occurring in the terminal field regions. The results illustrated that changes in shape cannot be accurately characterised with the methods employed here. The second examined the specific local changes in LGN shape, and whether there was a relationship between corresponding local changes in terminal field regions. No relationship could be demonstrated, but this was probably due to the fact that truly corresponding landmarks were not compared.

These approaches are probably the only way of demonstrating a direct relationship between the structure of the LGN and the formation of ocular distinct regions within the nucleus. It is therefore worth addressing some of the problems that have been highlighted by this work, in particular the issue of calculating more accurate transformation matrices.

In conclusion, this work has adopted an original and unique approach to analysing the dynamic anatomy of the developing visual system. It has been shown that the shape of the LGN is influenced mainly by innervating axons, but it has not been possible to determine whether changing nuclear shape affects segregation. Several 
observations have been made which indicate that the hypothesis should not be dismissed. 


\section{Bibliography.}

Angelucci, A., F. Clasca, E. Bricolo, K. S. Cramer and M. Sur (1997). "Experimentally induced retinal projections to the ferret auditory thalamus: development of clustered eye-specific patterns in a novel target." J Neurosci 17(6): 2040-55.

Antonelli, P. L. (1985). Non-Euclidean allometry and the growth of forests and corals. Mathematical Essays on Growth and the Emergence of Form. P. L. Antonelli. Edmonton, Alberta, University of Alberta Press.

Archer, S. M., M. W. Dubin and L. A. Stark (1982). “Abnormal development of kitten retino-geniculate connectivity in the absence of action potentials." Science 217(4561): 743-5.

Biesold, D., G. Bruckner and V. Mares (1976). “An autoradiographic study of gliogenesis in the rat lateral geniculate nucleus (LGN)." Brain Res 104(2): 295-301.

Bishop, P. O., W. Kozak, W. R. Levick and G. J. Vakkur (1962). "The determination of the projection of the visual field on to the lateral geniculate nucleus in the cat." $\underline{J}$. Physiol., Lond. 163: 503-539.

Boire, D., M. Herbin and M. Ptito (1997). "Retino-geniculate projections in the vervet monkey following hemispherectomy." Soc. Neurosci. Abs. 23: 1819.

Braendgaard, H. and H. J. Gundersen (1986). "The impact of recent stereological advances on quantitative studies of the nervous system." J Neurosci Methods 18(12): $39-78$.

Brechbühler, C., G. Gerig and O. Kübler (1995). "Parametrization of Closed Surfaces For 3-D Shape-Description." Computer Vision and Image Understanding 61(2): 154-170.

Brunso-Bechtold, J. K., D. Agee and A. J. Sweatt (1992). "Immunohistochemical evidence for transient expression of fibronectin in the developing dorsal lateral geniculate nucleus of the ferret." J Comp Neurol 315(3): 275-86. 
Brunso-Bechtold, J. K. and S. L. Vinsant (1990). "An ultrastructural and morphometric study of the effect of removal of retinal input on the development of the dorsal lateral geniculate nucleus.” J Comp Neurol 301(4): 585-603.

Campbell, G. and C. J. Shatz (1992). "Synapses formed by identified retinogeniculate axons during the segregation of eye input." J Neurosci 12(5): 1847-58.

Casagrande, V. A. and T. T. Norton (1991). Lateral geniculate nucleus: A review of its physiology and function. The Neural Basis of Visual Function. A. G. Leventhal. London, Macmillan Press. 4: 41-84.

Cavelieri, B. (1966). Geometria Delgi Indivisibili. Torino, Unione Tipografico, Editrice.

Chalupa, L. M. and C. J. Snider (1998). "Topographic specificity in the retinocollicular projection of the developing ferret: an anterograde tracing study." J Comp Neurol 392(1): 35-47.

Chalupa, L. M. and R. W. Williams (1984). “Organization of the cat's lateral geniculate nucleus following interruption of prenatal binocular competition." 3(2): 103-7.

Claps, A. and V. A. Casagrande (1990). "The distribution and morphology of corticogeniculate axons in ferrets." Brain Res 530(1): 126-9.

Cowan, W. M. (1973). Neuronal death as a regulative mechanism in the control of cell number in the nervous system. New York, Academic Press.

Cucchiaro, J. and R. W. Guillery (1984). "The development of the retinogeniculate pathways in normal and albino ferrets." Proc R Soc Lond B Biol Sci 223(1231): 141-64.

Cucchiaro, J. B. (1991). "Early development of the retinal line of decussation in normal and albino ferrets." J Comp Neurol 312(2): 193-206. 
Cucchiaro, J. B., D. J. Uhlrich and S. M. Sherman (1991). "Electron-microscopic analysis of synaptic input from the perigeniculate nucleus to the A-laminae of the lateral geniculate nucleus in cats." J Comp Neurol 310(3): 316-36.

Cullen, M. J. and I. R. Kaiserman-Abramof (1976). "Cytological organization of the dorsal lateral geniculate nuclei in mutant anophthalmic and postnatally enucleated mice." J Neurocytol 5(4): 407-24.

Dubin, M. W., L. A. Stark and S. M. Archer (1986). “A role for action-potential activity in the development of neuronal connections in the kitten retinogeniculate pathway." J Neurosci 6(4): 1021-36.

Eggermont, J. J. (1985). "Mathematical models for developmental changes.” Acta Otolaryngol Suppl 421: 102-7.

Eglen, S. (1995). Modelling the development of the cat lateral geniculate nucleus with Hebbian learning. Brighton, University of Sussex.

Elgeti, H., R. Elgeti and K. Fleischhauer (1976). "Postnatal growth of the dorsal lateral geniculate nucleus of the cat." Anat Embryol Berl 149(1): 1-13.

Enroth-Cugell, C. and J. G. Robson (1966). "The contrast sensitivity of retinal ganglion cells of the cat." J. Physiol. 187: 517-552.

Esguerra, M., A. W. Roe and M. Sur (1987). "Morphology of identified ferret LGN neurons characterized in vivo and in vitro." Soc. Neurosci. Abs. 13: 1434.

Finlay, B. L. and S. L. Pallas (1989). "Control of cell number in the developing mammalian visual system." Prog Neurobiol 32(3): 207-34.

Friedlander, M. J., C. S. Lin and S. M. Sherman (1979). "Structure of physiologically identified X and Y cells in the cat's lateral geniculate nucleus." Science 204(4397): 1114-7.

Galli, L. and L. Maffei (1988). "Spontaneous impulse activity of rat retinal ganglion cells in prenatal life." Science 242(4875): 90-1. 
Garey, L. J. and T. P. Powell (1971). "An experimental study of the termination of the lateral geniculo- cortical pathway in the cat and monkey." Proc R Soc Lond B Biol Sci 179(54): 41-63.

Garraghty, P. E., M. Sur and S. M. Sherman (1986a). "Role of competitive interactions in the postnatal development of $\mathrm{X}$ and $\mathrm{Y}$ retinogeniculate axons." $\mathrm{J}$ Comp Neurol 251(2): 216-39.

Garraghty, P. E., M. Sur, R. E. Weller and S. M. Sherman (1986b). "Morphology of retinogeniculate $\mathrm{X}$ and $\mathrm{Y}$ axon arbors in monocularly enucleated cats." $\underline{\mathrm{J} \text { Comp }}$ Neurol 251(2): 198-215.

Gill, P. E., W. Murray and M. H. Wright (1981). Practical Optimization. London, Academic Press.

Gonzalez, R. C. and R. E. Woods (1992). Digital image processing. Reading, Mass. ; Wokingham, Addison-Wesley.

Guillery, R. W. (1966). “A study of Golgi preparations from the dorsal lateral geniculate nucleus of the adult cat." J Comp Neurol 128(1): 21-50.

Guillery, R. W. (1989). "Early monocular enucleations in fetal ferrets produce a decrease of uncrossed and an increase of crossed retinofugal components: a possible model for the albino abnormality." J Anat 164: 73-84.

Guillery, R. W. and K. Herrup (1997). "Quantification without pontification: choosing a method for counting objects in sectioned tissues [comment]." J Comp Neurol 386(1): 2-7.

Guillery, R. W., A. S. LaMantia, J. A. Robson and K. Huang (1985). "The influence of retinal afferents upon the development of layers in the dorsal lateral geniculate nucleus of mustelids." J Neurosci 5(5): 1370-9.

Guillery, R. W. and M. D. Oberdorfer (1977). "A study of fine and coarse retino-fugal axons terminating in the geniculate $\mathrm{C}$ laminae and in the medial interlaminar nucleus of the mink." J Comp Neurol 176(4): 515-26. 
Gundersen, H. J. (1986). "Stereology of arbitrary particles. A review of unbiased number and size estimators and the presentation of some new ones, in memory of William R. Thompson.” J Microsc 143(Pt 1): 3-45.

Hebb, D. O. (1949). The Organization of Behaviour: A Neuropsychological Theory. Wiley, New York.

Henderson, Z., B. L. Finlay and K. C. Wikler (1988). "Development of ganglion cell topography in ferret retina." J Neurosci 8(4): 1194-205.

Hubel, D. H. and T. N. Wiesel (1965). "Binocular interaction in striate cortex of kittens reared with artificial squint." J. Neurophysiol. 28: 1041-1059.

Hutchins, J. B. and V. A. Casagrande (1990). "Development of the lateral geniculate nucleus: interactions between retinal afferent, cytoarchitectonic, and glial cell process lamination in ferrets and tree shrews." J Comp Neurol 298(1): 113-28.

Huxley, J. (1932). Problems of relative growth, Methuen \& Co.

Illing, R. B. and H. Wassle (1981). "The retinal projection to the thalamus in the cat: a quantitative investigation and a comparison with the retinotectal pathway." J Comp Neurol 202(2): 265-85.

Jeffery, G. (1984). "Retinal ganglion cell death and terminal field retraction in the developing rodent visual system." Brain Res 315(1): 81-96.

Jeffery, G. (1985). "Retinotopic order appears before ocular separation in developing visual pathways." Nature 313(6003): 575-6.

Jeffery, G. (1989). "Shifting retinal maps in the development of the lateral geniculate nucleus.” Brain Res Dev Brain Res 46(2): 187-96.

Jeffery, G. (1990). "The topographic relationship between shifting binocular maps in the developing dorsal lateral geniculate nucleus." Exp Brain Res 82(2): 408-16. 
Johnson, J. K. and V. A. Casagrande (1993). "Prenatal development of axon outgrowth and connectivity in the ferret visual system." Vis Neurosci 10(1): 11730 .

Jones, E. G. (1985). The thalamus. New York, Plenum Press.

Kaas, J. H., R. W. Guillery and J. M. Allman (1972). "Some principles of organization in the dorsal lateral geniculate nucleus." Brain Behav Evol 6(1): 253-99.

Kalil, R. (1978). "Development of the dorsal lateral geniculate nucleus in the cat." $\mathrm{J}$ Comp Neurol 182(2): 265-91.

Kalil, R. (1980). "Quantitative study of the effects of monocular enucleation and deprivation on cell growth in the dorsal lateral geniculate nucleus of the cat." $\underline{\mathbf{J}}$ Comp Neurol 189(3): 483-524.

Keesing, R., D. G. Stork and C. J. Shatz (1993). "Retinogeniculate development: the role of competition and correlated retinal activity." Advances in Neural Information Processing Systems I4: 91-97.

Kelemen, A., S. Székely and G. Gerig (1997). Three-dimensional Model-based Segmentation. Zurich, Communication Technology Laboratory, ETH: 1-10.

Kuhl, F. and C. Giardina (1982). "Elliptic Fourier features of a closed contour." Computer Graphics and Image Processing 18(3): 236-258.

Lee, D. Y. and J. G. Malpeli (1994). "Global form and singularity - modeling the blind spots role in lateral geniculate morphogenesis." Science 263(5151): 1292-1294.

LeVay, S. and D. Ferster (1977). "Relay cell classes in the lateral geniculate nucleus of the cat and the effects of visual deprivation." J Comp Neurol 172(4): 563-84.

Linden, D. C., R. W. Guillery and J. Cucchiaro (1981). "The dorsal lateral geniculate nucleus of the normal ferret and its postnatal development." J Comp Neurol 203(2): 189-211. 
Marcus, L. F., M. Corti, A. Loy, G. J. P. Naylor and D. E. Slice, Eds. (1996). Advances in Morphometrics. NATO ASI Series A: Life Sciences. New York, Plenum.

Marsden, J. E. and A. J. Tromba (1996). Vector Calculus. New York, W.H. Freeman and Company.

Martin, P. R. (1986). "The projection of different retinal ganglion cell classes to the dorsal lateral geniculate nucleus in the hooded rat." Exp Brain Res 62(1): 77-88.

Martinou, J. C., M. Dubois-Dauphin, J. K. Staple, I. Rodriguez, H. Frankowski, M. Missotten, P. Albertini, D. Talabot, S. Catsicas, C. Pietra and et al. (1994). "Overexpression of BCL-2 in transgenic mice protects neurons from naturally occurring cell death and experimental ischemia." Neuron 13(4): 1017-30.

Meister, M., R. O. Wong, D. A. Baylor and C. J. Shatz (1991). "Synchronous bursts of action potentials in ganglion cells of the developing mammalian retina." Science 252(5008): 939-43.

Minkowski, M. (1913). "Experimentelle Untersuchungen über die Beziehungen der Grosshirnrinde und der Netzhaut zu den primären Zentren, besonders zum Corpus geniculatum externum." Arb. Hirnanat. Inst. Zürich 7: 255-362.

Minkowski, M. (1920). "Uber den Verlauf, die Endigung und die zentrale Repräsentation von gekreutzten Sehnervenfasern bei einigen Säugertieren und beim Mensch." Schweiz. Arch. Neurol. Psychiat. 6: 201-252.

Moore, C. L., R. Kalil and W. Richards (1976). "Development of myelination in optic tract of the cat." J Comp Neurol 165(2): 125-36.

Morgan, J. E. (1986). The organization of the retinogeniculate pathways in normal and neonatally enucleated pigmenyed and albino ferrets. $\mathrm{PhD}$ Thesis, St. John's College. Oxford, Oxford University.

Morgan, J. E., Z. Henderson and I. D. Thompson (1987). "Retinal decussation patterns in pigmented and albino ferrets." Neuroscience 20(2): 519-35. 
Muller, C. (1966). Spherical Harmonics. Berlin-Heidelberg-New York, SpringerVerlag.

O'Leary, D. D., J. W. Fawcett and W. M. Cowan (1986). "Topographic targeting errors in the retinocollicular projection and their elimination by selective ganglion cell death." J Neurosci 6(12): 3692-705.

Olucha, F., F. Martinez-Garcia and C. Lopez-Garcia (1985). “A new stabilizing agent for the tetramethyl benzidine (TMB) reaction product in the histochemical detection of horseradish peroxidase (HRP)." J Neurosci Methods 13(2): 131-8.

Payne, B. R. and S. G. Lomber (1996). “Age dependent modification of cytochrome oxidase activity in the cat dorsal lateral geniculate nucleus following removal of primary visual cortex." Vis Neurosci 13(5): 805-16.

Price, D. J. and J. E. Morgan (1987). "Spatial properties of neurones in the lateral geniculate nucleus of the pigmented ferret." Exp Brain Res 68(1): 28-36.

Raghavan, R., W. Lawton, S. R. Ranjan and R. R. Viswanathan (1997). "A continuum mechanics-based model for cortical growth." J Theor Biol 187: 285-296.

Rakic, P. (1976). "Prenatal genesis of connections subserving ocular dominance in the rhesus monkey." Nature 261(5560): 467-71.

Rakic, P. (1977). "Genesis of the dorsal lateral geniculate nucleus in the rhesus monkey: site and time of origin, kinetics of proliferation, routes of migration and pattern of distribution of neurons." J Comp Neurol 176(1): 23-52.

Rakic, P. (1981). "Development of visual centers in the primate brain depends on binocular competition before birth." Science 214(4523): 928-31.

Rakic, P. and K. P. Riley (1983a). "Overproduction and elimination of retinal axons in the fetal rhesus monkey." Science 219(4591): 1441-4.

Rakic, P. and K. P. Riley (1983b). "Regulation of axon number in primate optic nerve by prenatal binocular competition." Nature 305(5930): 135-7. 
Ramoa, A. S., G. Campbell and C. J. Shatz (1989). "Retinal ganglion beta cells project transiently to the superior colliculus during development." Proc Natl Acad Sci U S A 86(6): 2061-5.

Richman, D. P., R. M. Stewart, J. W. Hutchinson and V. S. Caviness (1975). “Mechanical model of brain convolutional development." Science 189: 18-21.

Robinson, S. R. and B. Dreher (1990). "The visual pathways of eutherian mammals and marsupials develop according to a common timetable." Brain Behav Evol 36(4): 177-95.

Robson, J. A. and E. E. Geisert, Jr. (1994). "Expression of a keratin sulfate proteoglycan during development of the dorsal lateral geniculate nucleus in the ferret." J Comp Neurol 340(3): 349-60.

Rohlf, F. J. and F. L. Bookstein, Eds. (1990). Proceedings of the Michigan Morphometrics Workshop. Special Publication No. 2. Ann Arbor, Michigan, The University of Michigan Museum of Zoology.

Sanchez-Vives, M. V., T. Bal, U. Kim, M. von Krosigk and D. A. McCormick (1996). "Are the interlaminar zones of the ferret dorsal lateral geniculate nucleus actually part of the perigeniculate nucleus?" J Neurosci 16(19): 5923-41.

Sanderson, K. J. (1974). "Lamination of the dorsal lateral geniculate nucleus in carnivores of the weasel (Mustelidae), raccoon (Procyonidae) and fox (Canidae) families." J Comp Neurol 153(3): 238-66.

Sanes, J. R. (1989). "Extracellular matrix molecules that influence neural development." Annu Rev Neurosci 12: 491-516.

Shatz, C. J. (1983). "The prenatal development of the cat's retinogeniculate pathway." J Neurosci 3(3): 482-99.

Shatz, C. J. (1994). "Viktor Hamburger Award review. Role for spontaneous neural activity in the patterning of connections between retina and LGN during visual system development." Int J Dev Neurosci 12(6): 531-46. 
Shatz, C. J. (1996). "Emergence of order in visual system development.” Proc Natl Acad Sci U S A 93(2): 602-8.

Shatz, C. J. and P. A. Kirkwood (1984). "Prenatal development of functional connections in the cat's retinogeniculate pathway." J Neurosci 4(5): 1378-97.

Shatz, C. J. and M. P. Stryker (1988). "Prenatal tetrodotoxin infusion blocks segregation of retinogeniculate afferents." Science 242(4875): 87-9.

Sherman, S. M. (1985). "Development of Retinal Projections to the Cats Lateral Geniculate- Nucleus." Trends in Neurosciences 8(8): 350-355.

Simon, D. K. and D. D. O'Leary (1992). "Development of topographic order in the mammalian retinocollicular projection." J Neurosci 12(4): 1212-32.

Sretavan, D. and C. J. Shatz (1984). "Prenatal development of individual retinogeniculate axons during the period of segregation." Nature 308(5962): 845-8.

Sretavan, D. W. and C. J. Shatz (1986). "Prenatal development of retinal ganglion cell axons: segregation into eye-specific layers within the cat's lateral geniculate nucleus." J Neurosci 6(1): 234-51.

Stone, J., B. Dreher and A. Leventhal (1979). "Hierarchical and parallel mechanisms in the organization of visual cortex." Brain Res 180(3): 345-94.

Stryker, M. P. and S. S. L. (1984). "Physiological segregation of ocular dominance columns depends on the patterns of afferent electrical activity.” Invest. Ophthalmol. Vis. Sci. (Suppl.) 25: 278.

Stryker, M. P. and K. R. Zahs (1983). "On and off sublaminae in the lateral geniculate nucleus of the ferret." J Neurosci 3(10): 1943-51.

Sutton, J. K. and J. K. Brunso-Bechtold (1991). "A Golgi study of dendritic development in the dorsal lateral geniculate nucleus of normal ferrets." J Comp Neurol 309(1): 71-85. 
Sutton, J. K. and J. K. Brunso-Bechtold (1993). "Dendritic development in the dorsal lateral geniculate nucleus of ferrets in the postnatal absence of retinal input: a Golgi study." J Neurobiol 24(3): 317-34.

Thompson, I. and C. Holt (1989). "Effects of intraocular tetrodotoxin on the development of the retinocollicular pathway in the Syrian hamster." J Comp Neurol 282(3): 371-88.

Thompson, I. D., J. E. Morgan and Z. Henderson (1993). "The effects of monocular enucleation on ganglion cell number and terminal distribution in the ferret's retinal pathway." Eur J Neurosci 5(4): 357-67.

Thompson, S. D. A. W. (1917). On growth and form. Cambridge, Cambridge University Press.

Tzonev, S., J. Malpeli and K. Schulten (In press). "A three-dimensional model of the morphogenesis of the rhesus lateral geniculate nucleus." J. Computational Neuroscience.

Uhlrich, D. J., J. B. Cucchiaro, A. L. Humphrey and S. M. Sherman (1991). "Morphology and axonal projection patterns of individual neurons in the cat perigeniculate nucleus." J Neurophysiol 65(6): 1528-41.

Updyke, B. V. (1975). "The patterns of projection of cortical areas 17, 18, and 19 onto the laminae of the dorsal lateral geniculate nucleus in the cat." J Comp Neurol 163(4): 377-95.

Van Essen, D. C. (1997). "A tension-based theory of morphogenesis and compact wiring in the central nervous system." Nature 385(6614): 313-8.

Villena, A., F. Diaz, V. Requena, L. Vidal and I. Perez de Vargas (1989). "Quantitative study of the dorsal lateral geniculate nucleus of the rat during the postnatal development." J Hirnforsch 30(2): 185-90.

Weber, A. J. and R. E. Kalil (1987). "Development of corticogeniculate synapses in the cat." J Comp Neurol 264(2): 171-92. 
West, M. J. and H. J. Gundersen (1990). "Unbiased stereological estimation of the number of neurons in the human hippocampus." J Comp Neurol 296(1): 1-22.

White, C. A. and L. M. Chalupa (1991). Development of mammalian retinofugal pathways. Neuroanatomy of the Visual Pathways and their Development. B. Dreher and S. R. Robinson. London, Macmillan Press. 3: 129-149.

Wolfram, S. (1991). Mathematica: a system for doing mathematics by computer, Addison-Wesley.

Wong, R. O., M. Meister and C. J. Shatz (1993). "Transient period of correlated bursting activity during development of the mammalian retina." Neuron 11(5): 92338.

Yhip, J. P. and M. A. Kirby (1990). "Topographic organization of the retinocollicular projection in the neonatal rat." Vis Neurosci 4(4): 313-29.

Zhang, H. Y. and K. P. Hoffmann (1993). "Retinal projections to the pretectum, accessory optic system and superior colliculus in pigmented and albino ferrets." Eur J Neurosci 5(5): 486-500. 


\section{Appendix A.}

\section{Data from the normal animal.}

Table A.1 - LGN volume, cell density and total cell number as measured in the normal animal. Columns shown mean (SD), and $n$ indicates the number of individuals in each sample.

\begin{tabular}{cccccccc}
\hline Age & $n$ & \multicolumn{2}{c}{ Volume $\left(\mathrm{mm}^{3}\right)$} & \multicolumn{2}{c}{ Density $\left(\times 10^{6} \mathrm{~mm}^{-3}\right)$} & \multicolumn{2}{c}{ Number $\left(\times 10^{6}\right)$} \\
\hline P0 & 4 & 0.17 & $(0.02)$ & 1.39 & $(0.26)$ & 0.23 & $(0.03)$ \\
P2 & 1 & 0.24 & - & 1.47 & - & 0.35 & - \\
P5 & 4 & 0.39 & $(0.04)$ & 1.37 & $(0.15)$ & 0.52 & $(0.06)$ \\
P8 & 5 & 0.47 & $(0.04)$ & 1.05 & $(0.06)$ & 0.50 & $(0.06)$ \\
P10 & 4 & 0.52 & $(0.08)$ & 0.84 & $(0.10)$ & 0.44 & $(0.03)$ \\
P23 & 1 & 1.04 & - & 0.34 & - & 0.35 & - \\
P36 & 2 & 3.56 & $(0.17)$ & 0.11 & $(0.004)$ & 0.39 & $(0.006)$ \\
P47 & 2 & 3.84 & $(1.11)$ & 0.10 & $(0.004)$ & 0.38 & $(0.10)$ \\
P83 & 2 & 3.09 & $(0.42)$ & 0.10 & $(0.014)$ & 0.30 & $(0.001)$ \\
P140 & 2 & 3.26 & $(0.11)$ & 0.11 & $(0.008)$ & 0.37 & $(0.02)$ \\
\hline
\end{tabular}

Table A.2 - Cell areas in the LGN and thalamus $\left(\mu \mathrm{m}^{2}\right)$.

\begin{tabular}{ccccc}
\hline & \multicolumn{2}{c}{ LGN } & \multicolumn{2}{c}{ Thalamus } \\
\hline Age & Mean & S.D. & Mean & S.D. \\
\hline E38 & 26.84 & 6.68 & 32.66 & 8.68 \\
P0 & 32.93 & 7.17 & 34.86 & 8.24 \\
P5 & 37.77 & 8.19 & 41.62 & 10.20 \\
P8 & 42.27 & 13.73 & 44.73 & 17.68 \\
P10 & 42.50 & 10.62 & 57.22 & 18.29 \\
P23 & 68.94 & 21.94 & 91.73 & 32.54 \\
P47 & 103.46 & 37.93 & 137.41 & 48.61 \\
P140 & 77.19 & 38.90 & 86.04 & 43.61 \\
\hline
\end{tabular}




\section{Appendix $B$.}

\section{Data from the monocularly enucleated animal.}

Table B.1 - LGN volume ipsilateral and contralateral to a monocular enucleation ( $\mathrm{mm}^{3}, n=2$ for all cases).

\begin{tabular}{ccccc}
\hline & \multicolumn{2}{c}{ Ipsilateral } & \multicolumn{2}{c}{ Contralateral } \\
\hline Age & Mean & S.D. & Mean & S.D. \\
\hline P5 & 0.36 & 0.03 & 0.27 & 0.02 \\
P10 & 0.47 & 0.06 & 0.41 & 0.08 \\
P25 & 2.14 & 0.36 & 1.31 & 0.02 \\
P47 & 3.29 & 0.50 & 2.52 & 0.77 \\
\hline
\end{tabular}

Table B.2 - Cell density in the LGN ipsilateral and contralateral to a monocular enucleation $\left(\times 10^{6} \mathrm{~mm}^{-3}, n=2\right.$ for all cases $)$.

\begin{tabular}{ccccc}
\hline & \multicolumn{2}{c}{ Ipsilateral } & \multicolumn{2}{c}{ Contralateral } \\
\hline Age & Mean & S.D. & Mean & S.D. \\
\hline P5 & 1.15 & 0.15 & 1.09 & 0.15 \\
P10 & 0.87 & 0.10 & 0.91 & 0.08 \\
P25 & 0.14 & 0.004 & 0.14 & 0.01 \\
P47 & 0.07 & 0.002 & 0.08 & 0.01 \\
\hline
\end{tabular}

Table B.3 - Total cell number in the LGN ipsilateral and contralateral to a monocular enucleation ( $\times 10^{6}, n=2$ for all cases).

\begin{tabular}{cllll}
\hline & \multicolumn{2}{c}{ Ipsilateral } & \multicolumn{2}{c}{ Contralateral } \\
\hline Age & Mean & S.D. & Mean & S.D. \\
\hline P5 & 0.41 & 0.02 & 0.29 & 0.02 \\
P10 & 0.39 & 0.02 & 0.33 & 0.03 \\
P25 & 0.30 & 0.04 & 0.18 & 0.01 \\
P47 & 0.23 & 0.04 & 0.20 & 0.03 \\
\hline
\end{tabular}


Table B.4 - Cell areas in the LGN ipsilateral and contralateral to a monocular enucleation $\left(\mu \mathrm{m}^{2}\right)$.

\begin{tabular}{ccccc}
\hline & \multicolumn{2}{c}{ Ipsilateral } & \multicolumn{2}{c}{ Contralateral } \\
\hline Age & Mean & S.D. & Mean & S.D. \\
\hline P5 & 37.14 & 8.32 & 29.86 & 6.28 \\
P10 & 40.003 & 8.69 & 37.96 & 8.73 \\
P25 & 89.20 & 24.80 & 81.90 & 24.88 \\
P47 & 116.90 & 32.89 & 112.33 & 30.13 \\
\hline
\end{tabular}




\section{Appendix $C$.}

\section{Validity of the model representations.}

The models of LGN structure used throughout this study need to be representative of the actual anatomy. The most appropriate way to validate the models would be to image the LGN as a whole 3D structure, and to perform comparisons based on these data. However, current technology does not allow this. Alternatively, models generated from serial sections cut in different planes could be compared with the models shown here to establish their validity. Unfortunately, both computer processing time and tissue was limited, which made this option unfeasible.

The method employed to validate the models was to compare cross-sections from the models with published images of the ferret LGN in planes of sections which did not correspond to that of the original dataset. Unfortunately, most developmental work on ferret LGN has been performed in horizontal sectioned material, and therefore comparisons with horizontal sections from the models would be useless, as the models originate from sections in this plane. However, some electrophysiological experiments which require the visualisation of electrode penetrations do provide sagittal and coronal sections of the adult ferret, and these therefore provided the necessary validation.

Figure C1 shows a sagittal (from Morgan, 1986) and coronal (from Stryker and Zahs, 1983) section from the LGN of adult ferrets. Accompanying these images are cross-sections from a P140 model, and a generic adult model (constructed from the mean of the P47, P83, P111, and P140 models) taken from the same planes and at similar levels within the nucleus. 
The general form of the model cross-sections is very similar to the photomicrographs; namely the coronal sections show the characteristic ' $\mathrm{C}$ ' shape, and the sagittal sections show the dorsal ledge extending rostrally. Assuming that the sections in the micrographs represent true coronal and sagittal planes, there is a slight alignment error implied in the reconstruction of the $3 \mathrm{D}$ models. The coronal section from Stryker (1983), shows a distinct inclination medially, away from the dorso-ventral plane, while the model cross sections are aligned parallel with this axis. Similarly, the sagittal section from Morgan (1986) shows a caudal inclination away from the dorsoventral axis, while the model cross-sections are again aligned with this axis.

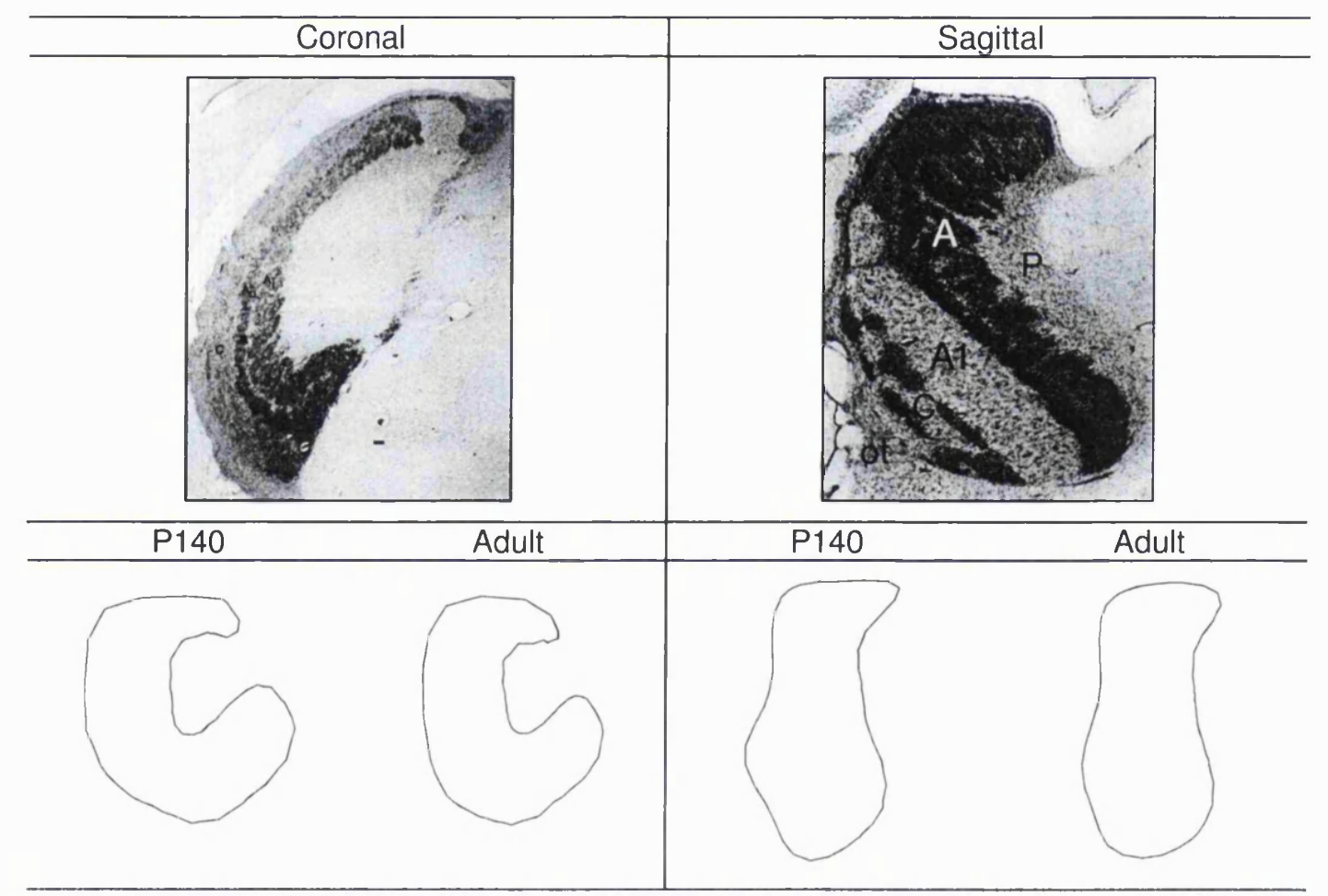

Figure C1 - Sagittal (from Morgan, 1986) and coronal (from Stryker and Zahs, 1983) sections from the LGN of adult ferrets. Accompanying these images are cross-sections from a P140 model, and a generic adult model (from the mean of the P47, P83, P111, and P140 models) in the same planes and at similar levels within the nucleus. Dorsal is up in all cases, medial to the right for the coronals, and rostral to the right for the sagittals. The figure illustrates a slight alignment error introduced in the reconstruction stage of the modelling process. 
The paucity of images in these planes, has meant that only adult data could be compared. Nevertheless, the comparisons show that gross features of the adult nucleus are represented by the models. Since all the models were generated with the same protocol, it must be assumed that this possible alignment error is present in all models. But the analysis presented in this study is comparative between models, and thus the results should be considered valid. Future work in this area should probably employ fudical marks in the tissue prior to sectioning, which would then provide an accurate means for alignment. However, it is difficult to envisage how this might be achieved given the size of the structures analysed here. 